\title{
Synthesis of Cyclopropene $\alpha$-Amino Acids via Enantioselective Desymmetrization
}

Fan Zhang and Joseph M. Fox*

Brown Laboratories, Department of Chemistry and Biochemistry

University of Delaware, Newark, DE 19716

\section{Supporting Information}

Experimental section

page 1

NMR spectrum

${ }^{1} \mathrm{H}$ and ${ }^{13} \mathrm{C}$ NMR spectrum of dimethyl 2-isopropyl-cycloprop-2-ene-1,1-dicarboxylic ester page 37

${ }^{1} \mathrm{H}$ and ${ }^{13} \mathrm{C}$ NMR spectrum of dimethyl 2-(4-fluorophenyl)-cycloprop-2-ene-1,1dicarboxylic ester page 38

${ }^{1} \mathrm{H}$ and ${ }^{13} \mathrm{C}$ NMR spectrum of dimethyl 2-isobutyl-cycloprop-2-ene-1,1-dicarboxylic ester page 39

${ }^{1} \mathrm{H}$ and ${ }^{13} \mathrm{C}$ NMR spectrum of dimethyl 2-methoxymethyl-cycloprop-2-ene-1,1-dicarboxylic ester page 40

${ }^{1} \mathrm{H}$ and ${ }^{13} \mathrm{C}$ NMR spectrum of dimethyl 2-trityloxymethyl-cycloprop-2-ene-1,1dicarboxylic ester

${ }^{1} \mathrm{H}$ and ${ }^{13} \mathrm{C}$ NMR spectrum of $\mathbf{1 a}$ dica
of $1 \mathbf{a}$
$\mathbf{1 b}$

page 41

page 42

${ }^{1} \mathrm{H}$ and ${ }^{13} \mathrm{C}$ NMR spectrum of $\mathbf{1 b}$ page 43

${ }^{1} \mathrm{H}$ and ${ }^{13} \mathrm{C}$ NMR spectrum of $\mathbf{1 c}$ page 44 ${ }^{1} \mathrm{H}$ and ${ }^{13} \mathrm{C}$ NMR spectrum of $\mathbf{1 d}$ page 45 ${ }^{1} \mathrm{H}$ and ${ }^{13} \mathrm{C}$ NMR spectrum of $\mathbf{1 e}$ page 46

${ }^{1} \mathrm{H}$ and ${ }^{13} \mathrm{C}$ NMR spectrum of $\mathbf{1 f}$ page 47 ${ }^{1} \mathrm{H}$ and ${ }^{13} \mathrm{C}$ NMR spectrum of $\mathbf{1 g}$ page 48 ${ }^{1} \mathrm{H}$ and ${ }^{13} \mathrm{C}$ NMR spectrum of $\mathbf{4 a}$ page 49 ${ }^{1} \mathrm{H}$ and ${ }^{13} \mathrm{C}$ NMR spectrum of $\mathbf{4 b}$ page 50 ${ }^{1} \mathrm{H}$ and ${ }^{13} \mathrm{C}$ NMR spectrum of $\mathbf{4 c}$ ${ }^{1} \mathrm{H}$ and ${ }^{13} \mathrm{C}$ NMR spectrum of $\mathbf{4 d}$ ${ }^{1} \mathrm{H}$ and ${ }^{13} \mathrm{C}$ NMR spectrum of $\mathbf{4 e}$ page 51 page 52 page 53 ${ }^{1} \mathrm{H}$ and ${ }^{13} \mathrm{C}$ NMR spectrum of $\mathbf{4 f}$ page 54 ${ }^{1} \mathrm{H}$ and ${ }^{13} \mathrm{C}$ NMR spectrum of $\mathbf{4 g}$ ${ }^{1} \mathrm{H}$ and ${ }^{13} \mathrm{C}$ NMR spectrum of $\mathbf{5 a}$ (major) page 55 ${ }^{1} \mathrm{H}$ and ${ }^{13} \mathrm{C}$ NMR spectrum of $\mathbf{5 a}$ (minor) page 56 ${ }^{1} \mathrm{H}$ and ${ }^{13} \mathrm{C}$ NMR spectrum of $\mathbf{5 b}(\boldsymbol{R c y}$, Sox $)$ page 57 page 58 
${ }^{1} \mathrm{H}$ and ${ }^{19} \mathrm{~F}$ NMR spectrum of $\mathbf{5 b}(\boldsymbol{S c y}, \boldsymbol{S o x})$

page 59

${ }^{1} \mathrm{H}$ and ${ }^{13} \mathrm{C}$ NMR spectrum of $\mathbf{5 c}$ (major)

page 60

${ }^{1} \mathrm{H}$ and ${ }^{13} \mathrm{C}$ NMR spectrum of $\mathbf{5 c}$ (minor)

page 61

${ }^{1} \mathrm{H}$ and ${ }^{13} \mathrm{C}$ NMR spectrum of $\mathbf{5 d}$ (major)

page 62

${ }^{1} \mathrm{H}$ and ${ }^{19} \mathrm{~F}$ NMR spectrum of $\mathbf{5 d}$ (minor)

page 63

${ }^{1} \mathrm{H}$ and ${ }^{13} \mathrm{C}$ NMR spectrum of $\mathbf{5 e}$ (major)

page 64

${ }^{1} \mathrm{H}$ and ${ }^{13} \mathrm{C}$ NMR spectrum of $\mathbf{5 e}$ (minor)

page 65

${ }^{1} \mathrm{H}$ and ${ }^{13} \mathrm{C}$ NMR spectrum of $\mathbf{5 f}$ (major)

page 66

${ }^{1} \mathrm{H}$ and ${ }^{13} \mathrm{C}$ NMR spectrum of $\mathbf{5 f}$ (minor)

page 67

${ }^{1} \mathrm{H}$ and ${ }^{13} \mathrm{C}$ NMR spectrum of $\mathbf{5 g}$ (major)

page 68

${ }^{1} \mathrm{H}$ and ${ }^{13} \mathrm{C}$ NMR spectrum of $\mathbf{5 g}$ (minor)

page 69

${ }^{1} \mathrm{H}$ and ${ }^{13} \mathrm{C}$ NMR spectrum of $\mathbf{6 a}$

page 70

${ }^{1} \mathrm{H}$ and ${ }^{13} \mathrm{C}$ NMR spectrum of $\mathbf{6 b}$

page 71

${ }^{1} \mathrm{H}$ and ${ }^{13} \mathrm{C}$ NMR spectrum of $7 \mathbf{a}$

page 72

${ }^{1} \mathrm{H}$ and ${ }^{13} \mathrm{C}$ NMR spectrum of $\mathbf{7 b}$

page 73

${ }^{1} \mathrm{H}$ and ${ }^{13} \mathrm{C}$ NMR spectrum of $\mathbf{8 a}$

page 74

${ }^{1} \mathrm{H}$ and ${ }^{13} \mathrm{C}$ NMR spectrum of $\mathbf{1 0 a}$

page 75

${ }^{1} \mathrm{H}$ and ${ }^{13} \mathrm{C}$ NMR spectrum of $\mathbf{1 0 b}$

page 76

${ }^{1} \mathrm{H}$ and ${ }^{13} \mathrm{C}$ NMR spectrum of $\mathbf{9 a}$

page 77

${ }^{1} \mathrm{H}$ NMR spectrum at variable temperatures of $9 \mathbf{a}$

page 78

${ }^{1} \mathrm{H}$ and ${ }^{13} \mathrm{C}$ NMR spectrum of $\mathbf{1 1}$

page 79

${ }^{1} \mathrm{H}$ and ${ }^{13} \mathrm{C}$ NMR spectrum of $\mathbf{1 2}$

page 80

${ }^{1} \mathrm{H}$ and ${ }^{13} \mathrm{C}$ NMR spectrum of $\mathbf{1 3}$

page 81

${ }^{1} \mathrm{H}$ and ${ }^{13} \mathrm{C}$ NMR spectrum of $\mathbf{1 4}$

page 82

\section{HPLC data}

HPLC data for 5a

page 83

HPLC data for $\mathbf{5 b}$

page 84

HPLC data for $\mathbf{5 c}$

page 85

HPLC data for $\mathbf{5 d}$

page 86

HPLC data for $\mathbf{5 e}$

page 87

HPLC data for $\mathbf{5 f}$

page 88

HPLC data for $\mathbf{5 g}$

page 89

\section{X-ray}

Molecular diagram from x-ray analysis of $\mathbf{5 b}(\boldsymbol{R} \mathbf{c y}, \boldsymbol{S o x})$ depicted with ellipsoids page 90 


\section{Experimental Section}

General Considerations : All reactions were carried out in glassware that was flamedried under vacuum, and cooled under nitrogen. Anhydrous THF was purchased from EMD. $\mathrm{CH}_{2} \mathrm{Cl}_{2}$ was dried with a column packed with activated neutral Alumina. Triethylamine was distilled under nitrogen from $\mathrm{CaH}_{2}$. The other reagents were purchased from commercial sources. (S)-Phenyloxazolidione was purchased from Chemicrea Inc., Tokyo, JAPAN. Chromatography was performed on silica gel (ICN SiliTech 32-62D, 60尺). For ${ }^{13} \mathrm{C}$ NMR, multiplicities were distinguished using an APT pulse sequence: typical methylene and quaternary carbons appear 'up' (u); methane and methyl carbons 'down' (dn). Exceptions are methine carbons of cyclopropenes, which usually have the same phase as 'normal' methylenes and quaternary carbons. 'dm' refers to 'doublet of multiplets', and is used to describe coupling patterns of pentafluorophenyl groups, in which only the one-bond C-F coupling constants are large and distinguishable. Diastereomeric ratios were determined using normal phase silica HPLC columns. Yields refer to isolated yields (average of 2 runs) of compounds estimated to be $>95 \%$ pure as determined by ${ }^{1} \mathrm{H}$ NMR unless noted otherwise. There is restricted rotation at $\mathrm{rt}$ on the ${ }^{1} \mathrm{H}$ NMR timescale for carbamate esters of cyclopropene $\alpha$-amino acids. This was demonstrated in a variable temperature experiment for 9a. Thus, two peaks (assignable to the alkene hydrogen) at $7.00 \mathrm{ppm}$ and $6.76 \mathrm{ppm}$ were found to coalesce when the temperature of the sample was increased from rt to $357 \mathrm{~K}$. The VT NMR data are shown in the Supporting Information. 


\section{Dimethyl 2-isopropyl-cycloprop-2-ene-1,1-dicarboxylic ester}

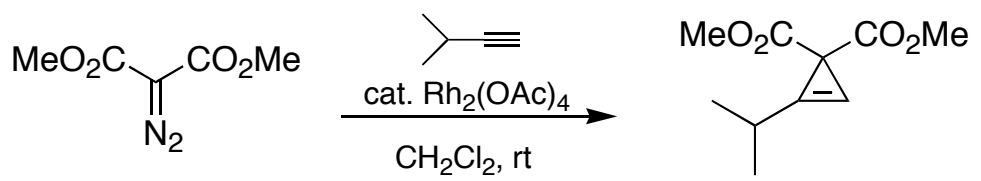

A solution of dimethyl diazomalonate $(2.0 \mathrm{~g}, 12.66 \mathrm{mmol})$ in $\mathrm{CH}_{2} \mathrm{Cl}_{2}(2 \mathrm{~mL})$ was added at $\mathrm{rt}$ via syringe pump $(0.5 \mathrm{~mL} / \mathrm{h})$ to a stirred mixture of 3-methyl-1-butyne $(1.8 \mathrm{~g}, 25.32$ $\mathrm{mmol})$ and $\mathrm{Rh}_{2}(\mathrm{OAc})_{4}(34 \mathrm{mg}, 0.076 \mathrm{mmol})$. After the syringe pump addition was completed, the solution was concentrated and chromatographed (10\% ether in hexanes) to give $1.79 \mathrm{~g} \mathrm{(72 \% )}$ of the title compound as pale yellow oil. A similar reaction gave $1.75 \mathrm{~g}$ (70\%) of the title compound. ${ }^{1} \mathrm{H}$ NMR $\left(400 \mathrm{MHz} \mathrm{CDCl}_{3}\right) \delta: 6.33(\mathrm{~d}, J=1.4 \mathrm{~Hz}, 1 \mathrm{H}), 3.71$

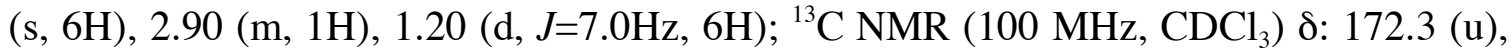
$119.4(\mathrm{u}), 92.6(\mathrm{u}), 52.5(\mathrm{dn}), 33.1(\mathrm{u}), 25.7(\mathrm{dn}), 20.3(\mathrm{dn})$; IR (neat, $\left.\mathrm{cm}^{-1}\right): 1729,1435$, 1280, 1241, 1190, 1099, 1061, 997, 948, 753; HRMS (CI) m/z: [M+H] calcd for $\mathrm{C}_{10} \mathrm{H}_{14} \mathrm{O}_{4}$ : 199.0970; found: 199.0962 .

\section{Dimethyl 2-(4-fluorophenyl)-cycloprop-2-ene-1,1-dicarboxylic ester}

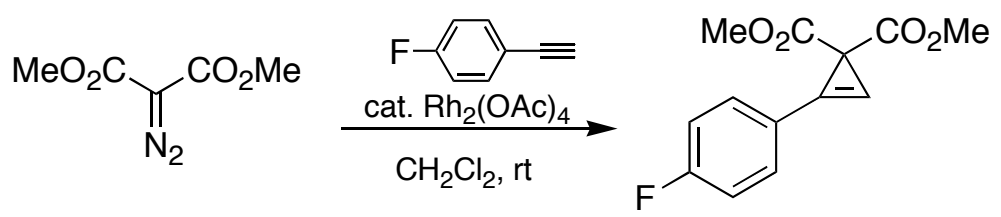

A solution of dimethyl diazomalonate $(0.66 \mathrm{~g}, 4.17 \mathrm{mmol})$ in $\mathrm{CH}_{2} \mathrm{Cl}_{2}(2 \mathrm{~mL})$ was added at $\mathrm{rt}$ via syringe pump $(0.5 \mathrm{~mL} / \mathrm{h})$ to a stirred mixture of 1-ethynyl-4-fluorobenzene $(1.0$ $\mathrm{g}, 8.33 \mathrm{mmol})$ and $\mathrm{Rh}_{2}(\mathrm{OAc})_{4}(11.1 \mathrm{mg}, 0.025 \mathrm{mmol})$ in $\mathrm{CH}_{2} \mathrm{Cl}_{2}(3 \mathrm{~mL})$. After the syringe pump addition was completed, the solution was concentrated and chromatographed (20\% ether in hexanes) to give $0.75 \mathrm{~g}(72 \%)$ of the title compound as pale yellow solid. A 
similar reaction gave $0.77 \mathrm{~g}(74 \%)$ of the title compound. mp: 59.5-61 ${ }^{\circ} \mathrm{C} ;{ }^{1} \mathrm{H}$ NMR (400

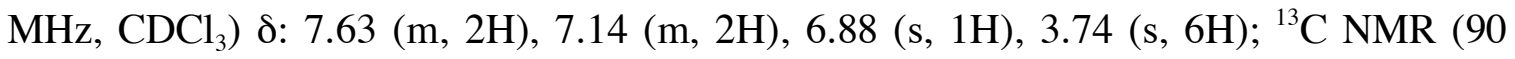
$\left.\mathrm{MHz}, \mathrm{CDCl}_{3}\right)$ d: $171.2(\mathrm{u}), 164.1\left(\mathrm{u}, \mathrm{d}, J_{\mathrm{FC}}=252 \mathrm{~Hz}\right), 132.6\left(\mathrm{dn}, \mathrm{d}, J_{\mathrm{FC}}=9 \mathrm{~Hz}\right), 120.5(\mathrm{u}, \mathrm{d}$, $\left.J_{\mathrm{FC}}=3.6 \mathrm{~Hz}\right), 116.4\left(\mathrm{dn}, \mathrm{d}, J_{\mathrm{FC}}=22 \mathrm{~Hz}\right), 111.5(\mathrm{u}), 95.0\left(\mathrm{u}, \mathrm{d}, J_{\mathrm{FC}}=2.5 \mathrm{~Hz}\right), 52.6(\mathrm{dn}), 33.0$ (u); ${ }^{19} \mathrm{~F}$ NMR (376 MHz, $\mathrm{CDCl}_{3}$ ) $\delta:-87.6(\mathrm{~m}) ; \mathrm{IR}\left(\right.$ neat, $\left.\mathrm{cm}^{-1}\right)$ : 1723, 1599, 1503, 1435, 1282, 1224, 1194, 1152, 1062, 974, 840, 740; HRMS (CI) m/z: $[\mathrm{M}+\mathrm{H}]$ calcd for $\mathrm{C}_{13} \mathrm{H}_{11} \mathrm{FO}_{4}: 251.0720$; found: 251.0708 .

\section{Dimethyl 2-isobutyl-cycloprop-2-ene-1,1-dicarboxylic ester}

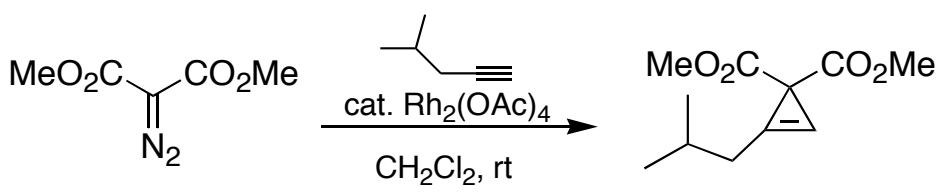

A solution of dimethyl diazomalonate $(2.0 \mathrm{~g}, 12.66 \mathrm{mmol})$ in $\mathrm{CH}_{2} \mathrm{Cl}_{2}(2 \mathrm{~mL})$ was added at $\mathrm{rt}$ via syringe pump $(0.5 \mathrm{~mL} / \mathrm{h})$ to a stirred mixture of 4-methyl-1-pentyne $(2.12 \mathrm{~g}$, $25.32 \mathrm{mmol})$ and $\mathrm{Rh}_{2}(\mathrm{OAc})_{4}(34 \mathrm{mg}, 0.076 \mathrm{mmol})$. After the syringe pump addition was completed, the solution was concentrated and chromatographed (10\% ether in hexanes) to give $2.0 \mathrm{~g}$ (74\%) of the title compound as pale yellow oil. A similar reaction starting from $1.65 \mathrm{~g}$ diazocompound gave $1.50 \mathrm{~g}(68 \%)$ of the title compound. ${ }^{1} \mathrm{H}$ NMR (400 $\mathrm{MHz}$, $\left.\mathrm{CDCl}_{3}\right) \delta: 6.40(\mathrm{t}, J=1.4 \mathrm{~Hz}, 1 \mathrm{H}), 3.71(\mathrm{~s}, 6 \mathrm{H}), 2.45(\mathrm{dd}, J=6.8,1.4 \mathrm{~Hz}, 2 \mathrm{H}), 1.97(\mathrm{~m}, 1 \mathrm{H})$, $0.98(\mathrm{~d}, J=6.7 \mathrm{~Hz}, 6 \mathrm{H}) ;{ }^{13} \mathrm{C}$ NMR $\left(100 \mathrm{MHz}, \mathrm{CDCl}_{3}\right) \delta: 172.3(\mathrm{u}), 114.1(\mathrm{u}), 94.5(\mathrm{u})$, $52.6(\mathrm{dn}), 33.4(\mathrm{u}), 32.7(\mathrm{u}), 27.1(\mathrm{dn}), 22.7(\mathrm{dn})$; IR (neat, $\left.\mathrm{cm}^{-1}\right):$ 1725, 1435, 1244, 1189, 1107, 1061, 982, 756; HRMS (CI) m/z: [M+H] calcd for $\mathrm{C}_{11} \mathrm{H}_{16} \mathrm{O}_{4}: 213.1126$; found: 213.1129. 


\section{Dimethyl 2-methoxymethyl-cycloprop-2-ene-1,1-dicarboxylic ester}

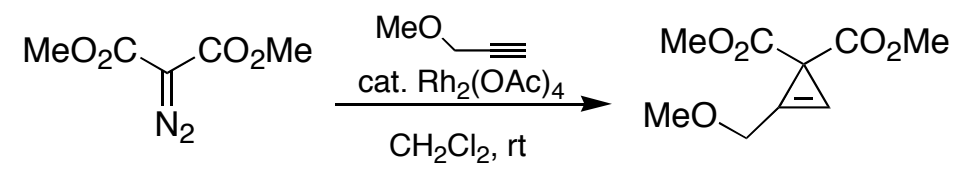

A solution of dimethyl diazomalonate $(2.0 \mathrm{~g}, 12.66 \mathrm{mmol})$ in $\mathrm{CH}_{2} \mathrm{Cl}_{2}(2 \mathrm{~mL})$ was added at $\mathrm{rt}$ via syringe pump $(0.5 \mathrm{~mL} / \mathrm{h})$ to a stirred mixture of 3 -methoxypropyne $(1.78 \mathrm{~g}$, $25.32 \mathrm{mmol})$ and $\mathrm{Rh}_{2}(\mathrm{OAc})_{4}(28 \mathrm{mg}, 0.063 \mathrm{mmol})$. After the syringe pump addition was completed, the solution was concentrated and chromatographed (10\% ethyl acetate in hexanes) to give $0.36 \mathrm{~g}(14 \%)$ of title compound as pale yellow oil. A similar reaction gave $0.41 \mathrm{~g}(16 \%)$ of the title compound. ${ }^{1} \mathrm{H} \mathrm{NMR}\left(400 \mathrm{MHz}, \mathrm{CDCl}_{3}\right)$ \&: 6.64 (t, $J=1.6 \mathrm{~Hz}, 1 \mathrm{H}), 4.43(\mathrm{~d}, J=1.6 \mathrm{~Hz}, 2 \mathrm{H}), 3.66(\mathrm{~s}, 6 \mathrm{H}), 3.36(\mathrm{~s}, 3 \mathrm{H}) ;{ }^{13} \mathrm{C} \mathrm{NMR}(100 \mathrm{MHz}$,

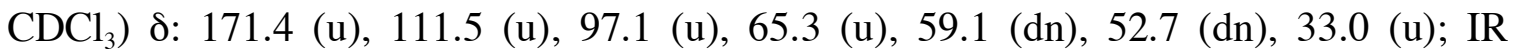
$\left(\mathrm{CH}_{2} \mathrm{Cl}_{2}, \mathrm{~cm}^{-1}\right) ; 1729,1435,1276,1247,1192,1109,1062 ; \operatorname{HRMS}(\mathrm{CI}) \mathrm{m} / \mathrm{z}:[\mathrm{M}+\mathrm{H}]$ calcd for $\mathrm{C}_{9} \mathrm{H}_{12} \mathrm{O}_{5}$ : 201.0763; found: 201.0763.

\section{Dimethyl 2-trityloxymethyl-cycloprop-2-ene-1,1-dicarboxylic ester}

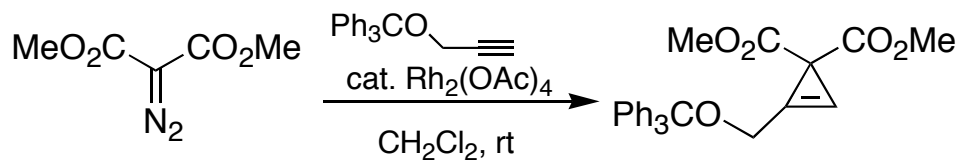

A solution of dimethyl diazomalonate $(163 \mathrm{mg}, 1.03 \mathrm{mmol})$ in $\mathrm{CH}_{2} \mathrm{Cl}_{2}(1 \mathrm{~mL})$ was added at $\mathrm{rt}$ via syringe pump $(0.25 \mathrm{~mL} / \mathrm{h})$ to a stirred mixture of 3-trityloxyl-1-propyne $(615 \mathrm{mg}$, $2.06 \mathrm{mmol})$ and $\mathrm{Rh}_{2}(\mathrm{OAc})_{4}(3 \mathrm{mg}, 0.006 \mathrm{mmol})$ in $\mathrm{CH}_{2} \mathrm{Cl}_{2}(5 \mathrm{~mL})$. After the syringe pump addition was completed, the solution was concentrated and chromatographed (10\% 
ethyl acetate in hexanes) to give $206 \mathrm{mg}(47 \%)$ of the title compound as white solid. A similar reaction starting from $815 \mathrm{mg}$ dimethyl diazomalonate gave $1.15 \mathrm{~g} \mathrm{(52 \% )}$ of the

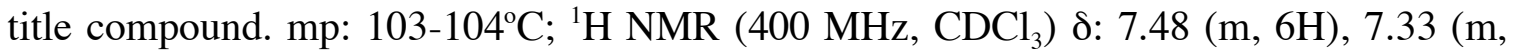
$6 \mathrm{H}), 7.28(\mathrm{~m}, 3 \mathrm{H}), 6.62(\mathrm{t}, J=1.5 \mathrm{~Hz}, 1 \mathrm{H}), 4.20(\mathrm{~d}, J=1.5 \mathrm{~Hz}, 2 \mathrm{H}), 3.74(\mathrm{~s}, 6 \mathrm{H}) ;{ }^{13} \mathrm{C} \mathrm{NMR}$

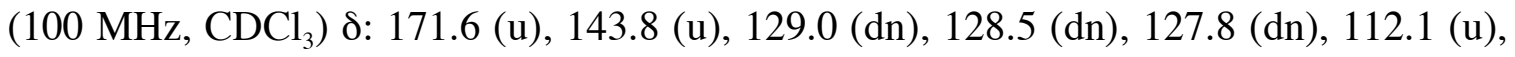
$96.4(\mathrm{u}), 88.0(\mathrm{u}), 58.0(\mathrm{u}), 52.8(\mathrm{dn}), 33.2(\mathrm{u})$; IR (neat, $\left.\mathrm{cm}^{-1}\right):$ 1740, 1712, 1434, 1276, 1223, 1188, 1155, 1068, 898, 849, 779, 765, 742, 707, 696, 642, 629; HRMS (ESI+) m/z: [M+Na] calcd for $\mathrm{C}_{27} \mathrm{H}_{24} \mathrm{O}_{5}: 451.1521$; found: 451.1543 .

\section{2-Butyl-cycloprop-2-ene-1,1-dicarboxylic acid (1a)}

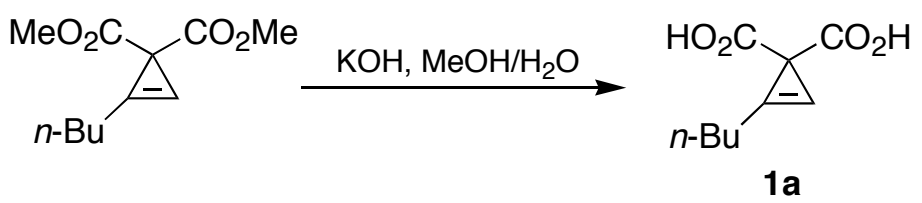

To a solution of dimethyl 2-butyl-cycloprop-2-ene-1,1-dicarboxylic ester $(1.77 \mathrm{~g}, 8.35$ mmol) in $\mathrm{MeOH}(40 \mathrm{~mL})$ was added $2 \mathrm{M}$ aq $\mathrm{KOH}(40 \mathrm{~mL})$ dropwise at $0^{\circ} \mathrm{C}$. The reaction mixture was allowed to stir at $\mathrm{rt}$ for $14 \mathrm{~h}$. The mixture was then concentrated to remove $\mathrm{MeOH}$ and diluted with water $(20 \mathrm{~mL})$. The mixture was washed with ethyl acetate $(3 \times 10$ $\mathrm{mL}$ ). The remaining aqueous layer was acidified with $10 \% \mathrm{HCl}(\mathrm{aq})$ and extracted with ethyl acetate $(3 \times 20 \mathrm{~mL})$. The organics were combined, dried over $\mathrm{Na}_{2} \mathrm{SO}_{4}$, filtered and concentrated to give $1.45 \mathrm{~g}(94 \%)$ of the title compound as white solid. A similar reaction starting from $5.25 \mathrm{~g}$ of diester gave $4.32 \mathrm{~g}(95 \%)$ of the title compound. $\mathrm{mp}: 95-96{ }^{\circ} \mathrm{C} ;{ }^{1} \mathrm{H}$ NMR (400 MHz, CD 3 CN) $\delta: 6.35(\mathrm{t}, J=1.5 \mathrm{~Hz}, 1 \mathrm{H}), 2.50(\mathrm{dt}, J=1.5 \mathrm{~Hz}, 7.2 \mathrm{~Hz}, 2 \mathrm{H}), 1.54$ $(\mathrm{m}, 2 \mathrm{H}), 1.40(\mathrm{~m}, 2 \mathrm{H}), 0.90(\mathrm{t}, J=7.3 \mathrm{~Hz}, 3 \mathrm{H}) ;{ }^{13} \mathrm{C} \mathrm{NMR}\left(90 \mathrm{MHz}, \mathrm{CD}_{3} \mathrm{CN}\right) \delta: 176.3(\mathrm{u})$, 
$110.8(\mathrm{u}), 90.8(\mathrm{u}), 30.6(\mathrm{u}), 28.6(\mathrm{u}), 23.2(\mathrm{u}), 22.1(\mathrm{u}), 13.3(\mathrm{dn})$; IR (neat, $\left.\mathrm{cm}^{-1}\right)$ : 1711, 1599, 1470, 1430, 1278, 1241, 1093, 1054, 915, 880, 797, 694, 674; HRMS (CI) m/z: [M-CO ${ }_{2}$ ] calcd for $\mathrm{C}_{9} \mathrm{H}_{12} \mathrm{O}_{4}: 140.0837$; found: 140.0833 .

2-Phenyl-cycloprop-2-ene-1,1-dicarboxylic acid (1b)

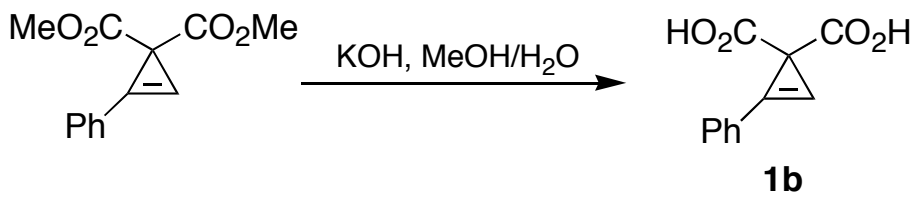

The procedure was identical to that used to prepare 1a, except that dimethyl 2-phenylcycloprop-2-ene-1,1-dicarboxylic ester $(3.80 \mathrm{~g}, 16.38 \mathrm{mmol}), \mathrm{MeOH}(40 \mathrm{~mL})$, and $2 \mathrm{M}$ aq $\mathrm{KOH}(40 \mathrm{~mL})$ were used. The crude product was recrystallized from $10 \%$ ethyl acetate and hexanes to give $2.9 \mathrm{~g}(87 \%)$ of title compound as pale yellow solid. A similar reaction starting from $2.19 \mathrm{~g}$ diester gave $1.62 \mathrm{~g}(84 \%)$ of the title compound. $\mathrm{mp}$ : 114$115{ }^{\circ} \mathrm{C} ;{ }^{1} \mathrm{H}$ NMR $\left(400 \mathrm{MHz}, \mathrm{CD}_{3} \mathrm{CN}\right) \delta: 7.58(\mathrm{~m}, 2 \mathrm{H}), 7.50(\mathrm{~m}, 3 \mathrm{H}), 6.99(\mathrm{~s}, 1 \mathrm{H}) ;{ }^{13} \mathrm{C}$

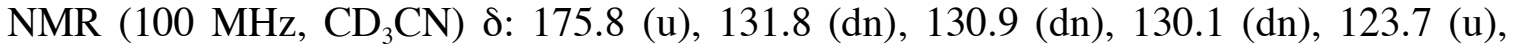
$108.8(\mathrm{u}), 94.8(\mathrm{u}), 31.0(\mathrm{u})$; IR (neat, $\left.\mathrm{cm}^{-1}\right)$ : 1713, 1642, 1633, 1598, 1470, 1433, 1289, 1247, 1196, 1180, 1093, 1055, 885, 825, 794, 752, 675, 622; HRMS (CI) m/z: [M-CO $]$ calcd for $\mathrm{C}_{11} \mathrm{H}_{8} \mathrm{O}_{4}$ : 160.0524 ; found: 160.0517 .

\section{2-Isopropyl-cycloprop-2-ene-1,1-dicarboxylic acid (1c)}

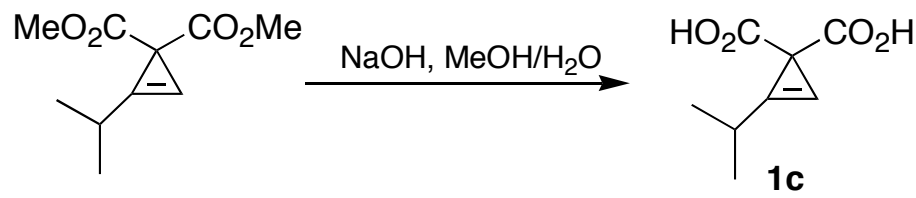


The procedure was identical to that used to prepare 1a, except that dimethyl 2-isopropylcycloprop-2-ene-1,1-dicarboxylic ester (1.00 g, $5.05 \mathrm{mmol}), \mathrm{MeOH}(30 \mathrm{~mL})$, and $1 \mathrm{M}$ aq $\mathrm{NaOH}(30 \mathrm{~mL})$ were used. The protocol gave $850 \mathrm{mg}(99 \%)$ of the title compound as white solid. A similar reaction gave $832 \mathrm{mg}$ (97\%) of the title compound. mp: 109$111^{\circ} \mathrm{C} ;{ }^{1} \mathrm{H}$ NMR $\left(400 \mathrm{MHz}, \mathrm{CD}_{3} \mathrm{CN}\right) \delta: 6.36(\mathrm{~d}, J=1.6 \mathrm{~Hz}, 1 \mathrm{H}), 2.90(\mathrm{~m}, 1 \mathrm{H}), 1.18(\mathrm{~d}$, $J=6.9 \mathrm{~Hz}, 6 \mathrm{H}) ;{ }^{13} \mathrm{C}$ NMR (100 MHz, $\left.\mathrm{CD}_{3} \mathrm{CN}\right) \delta: 177.0(\mathrm{u}), 115.6(\mathrm{u}), 90.2(\mathrm{u}), 31.1(\mathrm{u})$, $26.0(\mathrm{dn}), 20.1(\mathrm{dn}) ; \mathrm{IR}\left(\right.$ neat, $\left.\mathrm{cm}^{-1}\right): 1713,1606,1470,1429,1275,1244,1088,871,707$, 679; HRMS (CI) m/z: [M+H] calcd for $\mathrm{C}_{8} \mathrm{H}_{10} \mathrm{O}_{4}: 171.0657$; found: 171.0657 .

\section{2-(4-Fluorophenyl)-cycloprop-2-ene-1,1-dicarboxylic acid (1d)}

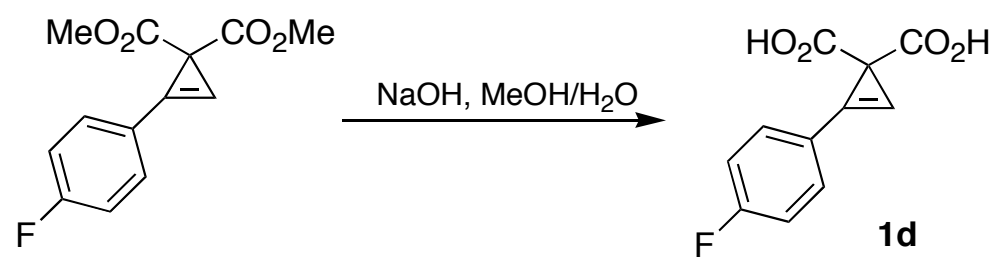

The procedure was identical to that used to prepare 1a, except that dimethyl 2-(4fluorophenyl)-cycloprop-2-ene-1,1-dicarboxylic ester (0.50 g, $2.0 \mathrm{mmol}), \mathrm{MeOH}(10$ $\mathrm{mL})$, and $1 \mathrm{M}$ aq $\mathrm{NaOH}(10 \mathrm{~mL})$ were used. The crude product was triturated with $10 \%$ ether in hexanes to give $0.43 \mathrm{~g}(96 \%)$ of the title compound as white solid. A similar reaction gave $0.42 \mathrm{~g}(95 \%)$ of the title compound. mp: $115-116^{\circ} \mathrm{C} ;{ }^{1} \mathrm{H}$ NMR $(400 \mathrm{MHz}$, acetone- $d 6) \delta: 7.72(\mathrm{~m}, 2 \mathrm{H}), 7.30(\mathrm{~m}, 3 \mathrm{H}) ;{ }^{13} \mathrm{C}$ NMR (100 MHz, acetone- $\left.d 6\right) \delta: 175.1(\mathrm{u})$, $164.6\left(\mathrm{u}, \mathrm{d}, J_{\mathrm{FC}}=249 \mathrm{~Hz}\right), 133.3\left(\mathrm{dn}, \mathrm{d}, J_{\mathrm{FC}}=9.0 \mathrm{~Hz}\right), 121.1\left(\mathrm{u}, \mathrm{d}, J_{\mathrm{FC}}=3.2 \mathrm{~Hz}\right), 117.1(\mathrm{dn}, \mathrm{d}$, $\left.J_{\mathrm{FC}}=22.6 \mathrm{~Hz}\right), 108.6(\mathrm{u}), 95.1\left(\mathrm{u}, \mathrm{d}, J_{\mathrm{FC}}=2.5 \mathrm{~Hz}\right), 31.5(\mathrm{u}) ;{ }^{19} \mathrm{~F}$ NMR (376 MHz, acetone-d6)

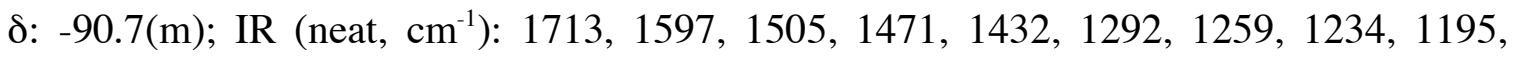


1159, 1093, 1057, 889, 833, 782; HRMS (CI) m/z: $\left[\mathrm{M}-\mathrm{CO}_{2}\right]$ calcd for $\mathrm{C}_{11} \mathrm{H}_{7} \mathrm{FO}_{4}$ : 178.0430; found: 178.0425 .

\section{2-Isobutyl-cycloprop-2-ene-1,1-dicarboxylic acid (1e)}

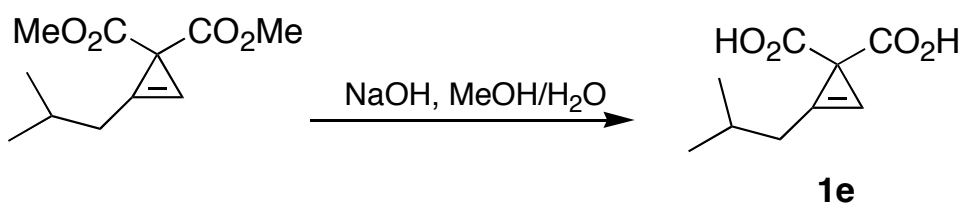

The procedure was identical to that used to prepare 1a, except that dimethyl 2-isobutylcycloprop-2-ene-1,1-dicarboxylic ester (1.05 g, $4.95 \mathrm{mmol}), \mathrm{MeOH}(30 \mathrm{~mL})$, and $1 \mathrm{M}$ aq $\mathrm{NaOH}(30 \mathrm{~mL})$ were used. The protocol gave $865 \mathrm{mg}(95 \%)$ of the title compound as white solid. A similar reaction gave $856 \mathrm{mg}$ (94\%) of the title compound. mp: 105$106^{\circ} \mathrm{C} ;{ }^{1} \mathrm{H}$ NMR (400 MHz, acetone- $\left.d 6\right) \delta: 6.71(\mathrm{t}, J=1.5 \mathrm{~Hz}, 1 \mathrm{H}), 2.47$ (dd, $J=6.7 \mathrm{~Hz}$, $1.5 \mathrm{~Hz}, 2 \mathrm{H}), 1.98(\mathrm{~m}, 1 \mathrm{H}), 1.03(\mathrm{~d}, J=6.7 \mathrm{~Hz}, 6 \mathrm{H}) ;{ }^{13} \mathrm{C} \mathrm{NMR}\left(100 \mathrm{MHz}, \mathrm{CD}_{3} \mathrm{CN}\right) \delta: 176.9$ (u), $110.4(u), 92.0(u), 32.9(u), 31.0(u), 27.4(d n), 22.3(d n)$; IR (neat, $\left.\mathrm{cm}^{-1}\right):$ 1723, 1714, 1601, 1469, 1454, 1426, 1278, 1240, 912, 879, 848, 799, 692, 672; HRMS (CI) m/z: [M- $\left.\mathrm{CO}_{2}+\mathrm{H}\right]$ calcd for $\mathrm{C}_{9} \mathrm{H}_{12} \mathrm{O}_{4}:$ 141.0915; found: 141.0911 .

\section{2-Methoxymethyl-cycloprop-2-ene-1,1-dicarboxylic acid (1f)}

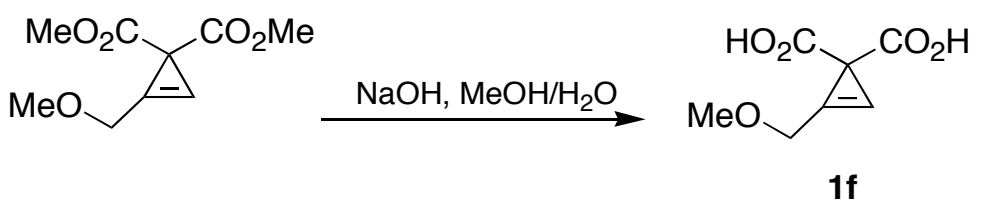

The procedure was identical to that used to prepare 1a, except that dimethyl 2methoxymethyl-cycloprop-2-ene-1,1-dicarboxylic ester $(326 \mathrm{mg}, 1.63 \mathrm{mmol})$, 
$\mathrm{MeOH}(10 \mathrm{~mL})$, and $1 \mathrm{M}$ aq $\mathrm{NaOH}(10 \mathrm{~mL})$ were used. The remaining acidified aqueous layer was extracted with ethyl acetate $(12 \times 10 \mathrm{~mL})$. The protocol gave $263 \mathrm{mg}(94 \%)$ of the title compound as pale yellow solid. A similar reaction starting from $0.41 \mathrm{~g}$ of diester gave $0.34 \mathrm{~g}(96 \%)$ of the title compound. mp:106-107 ${ }^{\circ} \mathrm{C} ;{ }^{1} \mathrm{H}$ NMR $\left(400 \mathrm{MHz}, \mathrm{CD}_{3} \mathrm{CN}\right)$ $\delta: 6.71(\mathrm{t}, J=1.7 \mathrm{~Hz}, 1 \mathrm{H}), 4.42(\mathrm{~d}, J=1.7 \mathrm{~Hz}, 2 \mathrm{H}), 3.40(\mathrm{~s}, 3 \mathrm{H}) ;{ }^{13} \mathrm{C}$ NMR $(100 \mathrm{MHz}$,

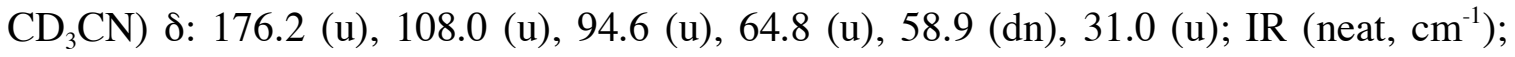
1705, 1593, 1426, 1288, 1246, 1194, 1107, 1056, 906, 839, 728, 675; HRMS (CI) m/z: [M- $\left.\mathrm{CO}_{2} \mathrm{H}\right]$ calcd for $\mathrm{C}_{7} \mathrm{H}_{8} \mathrm{O}_{5}: 127.0395$; found: 127.0393 .

\section{2-Trityloxymethyl-cycloprop-2-ene-1,1-dicarboxylic acid (1g)}

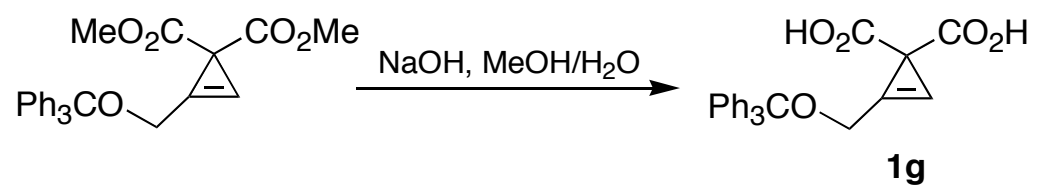

The procedure was identical to that used to prepare 1a, except that dimethyl 2trityloxymethyl-cycloprop-2-ene-1,1-dicarboxylic ester (156 mg, $0.36 \mathrm{mmol})$, THF (4 $\mathrm{mL})$, and $1 \mathrm{M}$ aq $\mathrm{NaOH}(2.2 \mathrm{~mL})$ were used. The protocol gave $128 \mathrm{mg}(89 \%)$ of the title compound as white solid. A similar reaction starting from $344 \mathrm{mg}$ of the diester gave 292 mg $(91 \%)$ of the title compound. mp: $115-116^{\circ} \mathrm{C} ;{ }^{1} \mathrm{H}$ NMR (400 MHz, THF- $\left.d 8\right) \delta: 12.20$ (bs, 2H), $7.45(\mathrm{~m}, 6 \mathrm{H}), 7.28(\mathrm{~m}, 6 \mathrm{H}), 7.22(\mathrm{~m}, 3 \mathrm{H}), 6.86(\mathrm{~d}, J=1.7 \mathrm{~Hz}, 1 \mathrm{H}), 4.07(\mathrm{~d}$, $J=1.7 \mathrm{~Hz}, 2 \mathrm{H}) ;{ }^{13} \mathrm{C}$ NMR (100 MHz, THF- $\left.d 8\right) \delta: 173.0(\mathrm{u}), 142.1(\mathrm{u}), 127.0(\mathrm{dn}), 126.2$ $(\mathrm{dn}), 125.5(\mathrm{dn}), 106.7(\mathrm{u}), 92.1(\mathrm{u}), 86.0(\mathrm{u}), 55.7(\mathrm{u}), 28.9(\mathrm{u}) ; \quad$ IR (neat, $\left.\mathrm{cm}^{-1}\right)$ : 1722, $1644,1611,1470,1446,1426,1280,1246,1080,1066,942,781,747,704,695,630$; HRMS (ESI+) m/z: [M+Na] calcd for $\mathrm{C}_{25} \mathrm{H}_{20} \mathrm{O}_{5}$ : 423.1208; found: 423.1225 . 
General procedure for making bis-pentafluorophenyl esters of cyclopropene biscarboxylic acids

The appropriate cyclopropene bis-carboxylic acid was dissolved either in $\mathrm{CH}_{2} \mathrm{Cl}_{2}$ or THF and cooled by an ice/water bath. To this solution was sequentially added an amine base (triethylamine or diisopropylethylamine) and pentafluorophenyl trifluoroacetate. The reaction mixture was allowed to stir for $30 \mathrm{~min}$, and was then concentrated. Rapid flash chromatography with $\mathrm{CH}_{2} \mathrm{Cl}_{2}$ gave the corresponding bis-pentafluorophenyl ester.

\section{bis-Pentafluorophenyl 2-butyl-cycloprop-2-ene-1,1-bis-carboxylic ester (4a)}

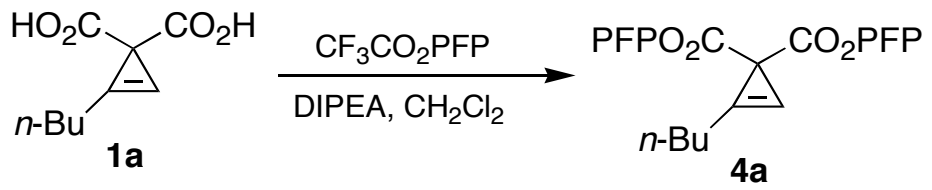

The general procedure for making bis-pentafluorophenyl esters with 1a $(184 \mathrm{mg}, 1.0$ mmol), pentafluorophenyl trifluoroacetate $(560 \mathrm{mg}, 2.0 \mathrm{mmol})$, diisopropylethylamine (310 mg, $2.4 \mathrm{mmol})$, and $\mathrm{CH}_{2} \mathrm{Cl}_{2}(10 \mathrm{~mL})$ gave $425 \mathrm{mg}$ (82\%) of the title compound as white solid. A similar reaction starting from $4.64 \mathrm{~g}$ of $1 \mathrm{a}$ gave $10.06 \mathrm{~g}(77 \%)$ of the title compound. mp: 37-39 ${ }^{\circ} \mathrm{C} ;{ }^{1} \mathrm{H}$ NMR (400 MHz, $\mathrm{CDCl}_{3}$ ) $\delta: 6.63$ (t, $\left.J=1.6 \mathrm{~Hz}, 1 \mathrm{H}\right), 2.74$ (dt, $J=1.5 \mathrm{~Hz}, 7.4 \mathrm{~Hz}, 2 \mathrm{H}), 1.73(\mathrm{~m}, 2 \mathrm{H}), 1.50(\mathrm{~m}, 2 \mathrm{H}), 0.96(\mathrm{t}, J=7.4 \mathrm{~Hz}, 3 \mathrm{H}) ;{ }^{13} \mathrm{C}$ NMR $(100$

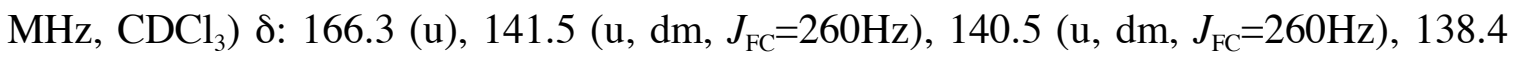
$\left(\mathrm{u}, \mathrm{dm}, J_{\mathrm{FC}}=250 \mathrm{~Hz}\right), 125.4(\mathrm{u}, \mathrm{m}), 114.3(\mathrm{u}), 92.5(\mathrm{u}), 32.0(\mathrm{u}), 28.5(\mathrm{u}), 24.0(\mathrm{u}), 22.6$ (u), $13.8(\mathrm{dn}) ;{ }^{19} \mathrm{~F}$ NMR (376 MHz, $\mathrm{CDCl}_{3}$ ) $\delta:-155.1$ (m), -162.7 (t, J=30.1Hz), -169.5 (m); IR (neat, $\left.\mathrm{cm}^{-1}\right): 1776,1518,1343,997$; HRMS (CI, $\left.\mathrm{NH}_{3}\right) \mathrm{m} / \mathrm{z}:\left[\mathrm{M}+\mathrm{NH}_{4}\right]$ calcd for $\mathrm{C}_{21} \mathrm{H}_{10} \mathrm{~F}_{10} \mathrm{O}_{4}$ : 534.0763; found: 534.0761 . 


\section{bis-Pentafluorophenyl 2-phenyl-cycloprop-2-ene-1,1-bis-carboxylic ester (4b)}

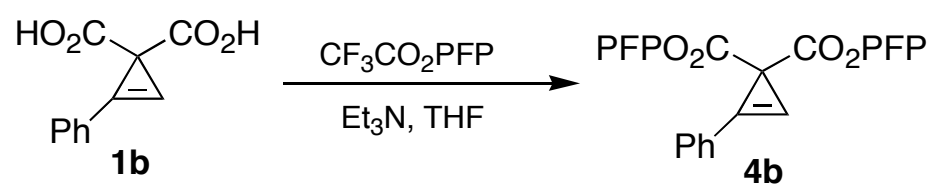

The general procedure for making bis-pentafluorophenyl esters with $\mathbf{1 b}$ (102 mg, 0.5 mmol), pentafluorophenyl trifluoroacetate $(280 \mathrm{mg}, 1.0 \mathrm{mmol}), \mathrm{Et}_{3} \mathrm{~N}(125 \mathrm{mg}, 1.2$ $\mathrm{mmol}$ ), and THF (3 mL) gave $220 \mathrm{mg}(82 \%)$ of the title compound as a pale yellow oil. A similar reaction gave $210 \mathrm{mg}(79 \%)$ of title compound. The purity of the compound was judged to be about $90 \%$ by ${ }^{1} \mathrm{H}$ NMR. Minor impurities were detected in the ${ }^{1} \mathrm{H}$ NMR at 7.33-7.53 ppm, $7.05 \mathrm{ppm}, 3.36-3.90 \mathrm{ppm}, 1.89 \mathrm{ppm}$. Because of the sensitivity of the compound toward silica gel, the compound was used in the next step without further purification. ${ }^{1} \mathrm{H}$ NMR $\left(400 \mathrm{MHz}, \mathrm{CDCl}_{3}\right) \delta: 7.78(\mathrm{~m}, 2 \mathrm{H}), 7.59(\mathrm{~m}, 3 \mathrm{H}), 7.17(\mathrm{~s}, 1 \mathrm{H}) ;{ }^{13} \mathrm{C}$

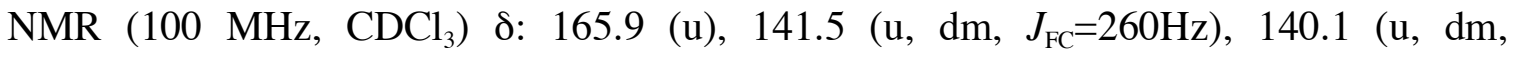
$\left.J_{\mathrm{FC}}=250 \mathrm{~Hz}\right), 138.3\left(\mathrm{u}, \mathrm{dm}, J_{\mathrm{FC}}=250 \mathrm{~Hz}\right), 132.2(\mathrm{dn}), 130.9(\mathrm{dn}), 129.8(\mathrm{dn}), 125.4(\mathrm{u}, \mathrm{m})$, $122.5(\mathrm{u}), 111.9(\mathrm{u}), 93.4(\mathrm{u}), 32.2(\mathrm{u}) ;{ }^{19} \mathrm{~F}$ NMR (376 MHz, $\mathrm{CDCl}_{3}$ ) $\delta:-154.6(\mathrm{~m}),-162.0$ (m), -169.0 (m); IR (neat, $\left.\mathrm{cm}^{-1}\right): 1778,1517,1244,1144,1088,993,908,765,732,695$; HRMS (ESI+) m/z: [M+Na] calcd for $\mathrm{C}_{23} \mathrm{H}_{6} \mathrm{~F}_{10} \mathrm{O}_{4}: 559.0004$; found: 559.0021 . 


\section{bis-Pentafluorophenyl 2-isopropyl-cycloprop-2-ene-1,1- bis-carboxylic ester (4c)}

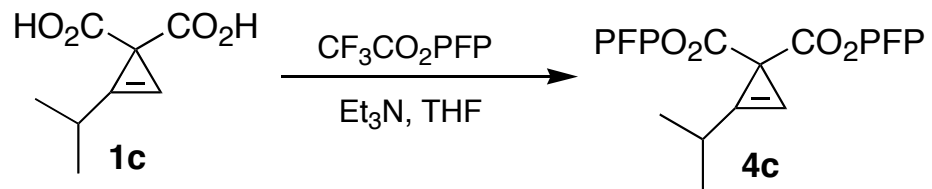

The general procedure for making bis-pentafluorophenyl esters with 1c $(85 \mathrm{mg}, 0.5$ $\mathrm{mmol}$ ), pentafluorophenyl trifluoroacetate $(280 \mathrm{mg}, 1.0 \mathrm{mmol}), \mathrm{Et}_{3} \mathrm{~N}(125 \mathrm{mg}, 1.2$ mmol), and THF (3 mL) gave $191 \mathrm{mg}(76 \%)$ of the title compound as colorless oil. A similar reaction gave $197 \mathrm{mg}(78 \%)$ of the title compound. ${ }^{1} \mathrm{H} \mathrm{NMR}\left(400 \mathrm{MHz}, \mathrm{CDCl}_{3}\right.$ ) $\delta: 6.62(\mathrm{~d}, J=1.6 \mathrm{~Hz}, 1 \mathrm{H}), 3.10(\mathrm{~m}, 1 \mathrm{H}), 1.35(\mathrm{~d}, J=7.0 \mathrm{~Hz}, 6 \mathrm{H}) ;{ }^{13} \mathrm{C}$ NMR $(100 \mathrm{MHz}$, $\left.\mathrm{CDCl}_{3}\right) \delta: 166.4(\mathrm{u}), 141.6\left(\mathrm{u}, \mathrm{dm}, J_{\mathrm{FC}}=250 \mathrm{~Hz}\right), 140.1\left(\mathrm{u}, \mathrm{dm}, J_{\mathrm{FC}}=230 \mathrm{~Hz}\right), 138.3(\mathrm{u}, \mathrm{dm}$, $\left.J_{\mathrm{FC}}=230 \mathrm{~Hz}\right), 125.4(\mathrm{u}, \mathrm{m}), 118.6(\mathrm{u}), 91.6(\mathrm{u}), 32.1(\mathrm{u}), 25.9(\mathrm{dn}), 20.0(\mathrm{dn}) ;{ }^{19} \mathrm{~F}$ NMR $\left(376 \mathrm{MHz}, \mathrm{CDCl}_{3}\right.$ ) $\delta:-154.8(\mathrm{~m}),-162.6(\mathrm{t}, J=33.8 \mathrm{~Hz}),-169.5(\mathrm{~m})$; IR (neat, $\mathrm{cm}^{-1}$ ): 1777, $1770,1519,1515,1241,1025,994 ; \operatorname{HRMS}\left(\mathrm{CI}, \mathrm{NH}_{3}\right) \mathrm{m} / \mathrm{z}:\left[\mathrm{M}+\mathrm{NH}_{4}\right]$ calcd for $\mathrm{C}_{20} \mathrm{H}_{8} \mathrm{~F}_{10} \mathrm{O}_{4}:$ 520.0606; found: 520.0607.

bis-Pentafluorophenyl 2-(4-fluorophenyl)-cycloprop-2-ene-1,1- bis-carboxylic ester (4d)

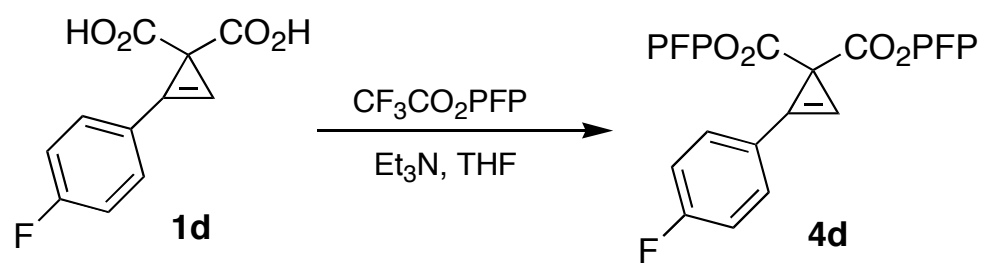

The general procedure for making bis-pentafluorophenyl esters with 1d $(111 \mathrm{mg}, 0.5$ $\mathrm{mmol}$ ), pentafluorophenyl trifluoroacetate $(280 \mathrm{mg}, 1.0 \mathrm{mmol}), \mathrm{Et}_{3} \mathrm{~N}(125 \mathrm{mg}, 1.2$ $\mathrm{mmol}$ ), and THF (3 mL) gave $185 \mathrm{mg}(67 \%)$ of the title compound as pale yellow oil. A similar reaction gave $176 \mathrm{mg}(64 \%)$ of the title compound. The purity of the compound 
was judged to be about $94 \%$ by ${ }^{1} \mathrm{H}$ NMR. Minor impurities were detected in the ${ }^{1} \mathrm{H}$ NMR at 7.40-7.60 ppm, $6.96 \mathrm{ppm}, 3.42-3.69 \mathrm{ppm}, 2.02 \mathrm{ppm}, 1.26 \mathrm{ppm}$. Because of the sensitivity of the compound toward silica gel, the compound was used in the next step

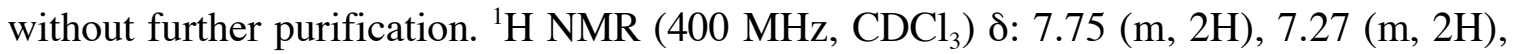
$7.12(\mathrm{~s}, 1 \mathrm{H}) ;{ }^{13} \mathrm{C}$ NMR $\left(100 \mathrm{MHz}, \mathrm{CDCl}_{3}\right) \delta: 165.8(\mathrm{u}), 165.1\left(\mathrm{u}, \mathrm{d}, J_{\mathrm{FC}}=250 \mathrm{~Hz}\right), 141.5$ $\left(\mathrm{u}, \mathrm{dm}, J_{\mathrm{FC}}=250 \mathrm{~Hz}\right), 140.1\left(\mathrm{u}, \mathrm{dm}, J_{\mathrm{FC}}=240 \mathrm{~Hz}\right), 138.4\left(\mathrm{u}, \mathrm{dm}, J_{\mathrm{FC}}=260 \mathrm{~Hz}\right), 133.2(\mathrm{dn}, \mathrm{d}$, $\left.J_{\mathrm{FC}}=9.1 \mathrm{~Hz}\right), 125.3(\mathrm{u}, \mathrm{m}), 118.8\left(\mathrm{u}, \mathrm{d}, J_{\mathrm{FC}}=3.3 \mathrm{~Hz}\right), 117.3\left(\mathrm{dn}, \mathrm{d}, J_{\mathrm{FC}}=22.3 \mathrm{~Hz}\right), 111.0(\mathrm{u})$, $93.1\left(\mathrm{u}, \mathrm{d}, J_{\mathrm{FC}}=2.4 \mathrm{~Hz}\right), \quad 32.3(\mathrm{u}) ;{ }^{19} \mathrm{~F} \mathrm{NMR}\left(376 \mathrm{MHz}, \mathrm{CDCl}_{3}\right) \quad \delta:-84.0(\mathrm{~m}),-154.6$ (m), $-161.6(\mathrm{t}, J=33.8 \mathrm{~Hz}),-168.6(\mathrm{~m})$; IR (neat, $\left.\mathrm{cm}^{-1}\right): 1779,1602,1517,1505,1240$, 1153, 1087, 995, 909, 841, 734; HRMS (ESI+) m/z: [M+Na] calcd for $\mathrm{C}_{23} \mathrm{H}_{5} \mathrm{~F}_{11} \mathrm{O}_{4}$ : 576.9910; found: 576.9913.

\section{bis-Pentafluorophenyl 2-isobutyl-cycloprop-2-ene-1,1- bis-carboxylic ester (4e)}

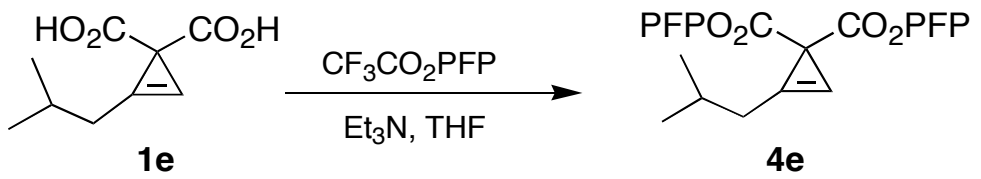

The general procedure for making bis-pentafluorophenyl esters with 1e (92 mg, 0.5 mmol), pentafluorophenyl trifluoroacetate $(280 \mathrm{mg}, 1.0 \mathrm{mmol}), \mathrm{Et}_{3} \mathrm{~N}(125 \mathrm{mg}, 1.2$ mmol), and THF (3 mL) gave $196 \mathrm{mg}(76 \%)$ of the title compound as white solid. A similar reaction gave $201 \mathrm{mg}(78 \%)$ of the title compound. mp: $81-82^{\circ} \mathrm{C} .{ }^{1} \mathrm{H}$ NMR $(400$

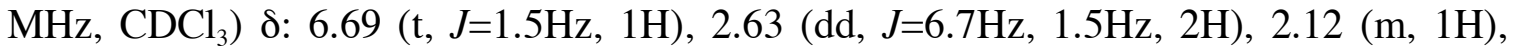
$1.08(\mathrm{~d}, J=6.7 \mathrm{~Hz}, 6 \mathrm{H}) ;{ }^{13} \mathrm{C} \mathrm{NMR}\left(100 \mathrm{MHz}, \mathrm{CDCl}_{3}\right) \delta: 166.3(\mathrm{u}), 141.5(\mathrm{u}, \mathrm{dm}$, $\left.J_{\mathrm{FC}}=260 \mathrm{~Hz}\right), 140.1\left(\mathrm{u}, \mathrm{dm}, J_{\mathrm{FC}}=240 \mathrm{~Hz}\right), 138.4\left(\mathrm{u}, \mathrm{dm}, J_{\mathrm{FC}}=240 \mathrm{~Hz}\right), 125.4(\mathrm{u}, \mathrm{m}), 113.5$ $(\mathrm{u}), 93.2(\mathrm{u}), 33.3(\mathrm{u}), \quad 32.0(\mathrm{u}), \quad 27.0(\mathrm{dn}), \quad 22.6(\mathrm{dn}) ;{ }^{19} \mathrm{~F}$ NMR $\left(376 \mathrm{MHz}, \mathrm{CDCl}_{3}\right)$ 
$\delta:-154.8(\mathrm{~m}),-162.4(\mathrm{t}, J=33.8 \mathrm{~Hz}),-169.3(\mathrm{~m})$; IR (neat, $\left.\mathrm{cm}^{-1}\right): 1778,1770,1519,1514$, 1245, 993; HRMS (CI, $\mathrm{NH}_{3}$ ) m/z: [M+NH 4 calcd for $\mathrm{C}_{21} \mathrm{H}_{10} \mathrm{~F}_{10} \mathrm{O}_{4}: 534.0763$; found: 534.0751.

bis-Pentafluorophenyl 2-methoxymethyl-cycloprop-2-ene-1,1-bis-carboxylic ester (4f)

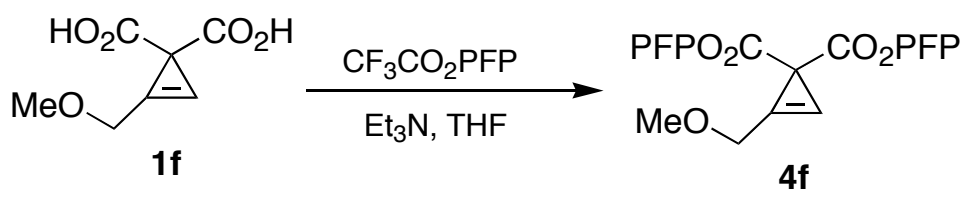

The general procedure for making bis-pentafluorophenyl esters with 1f $(85 \mathrm{mg}, 0.5$ mmol), pentafluorophenyl trifluoroacetate $(280 \mathrm{mg}, 1.0 \mathrm{mmol}), \mathrm{Et}_{3} \mathrm{~N}(125 \mathrm{mg}, 1.2$ mmol), and THF ( $3 \mathrm{~mL}$ ) gave $164 \mathrm{mg}(65 \%)$ of the title compound as pale yellow oil. A similar reaction gave $150 \mathrm{mg}(60 \%)$ of the title compound. ${ }^{1} \mathrm{H}$ NMR $\left(400 \mathrm{MHz}, \mathrm{CDCl}_{3}\right.$ ) $\delta: 6.96(\mathrm{t}, J=1.7 \mathrm{~Hz}, 1 \mathrm{H}), 4.64(\mathrm{~d}, J=1.6 \mathrm{~Hz}, 2 \mathrm{H}), 3.50(\mathrm{~s}, 3 \mathrm{H}) ;{ }^{13} \mathrm{C}$ NMR $(100 \mathrm{MHz}$, $\left.\mathrm{CDCl}_{3}\right) \delta: 165.6(\mathrm{u}), 141.5(\mathrm{u}, \mathrm{dm}, 250 \mathrm{~Hz}), 140.1(\mathrm{u}, \mathrm{dm}, J=250 \mathrm{~Hz}), 138.7(\mathrm{u}, \mathrm{dm}$, $J=250 \mathrm{~Hz}), 125.3(\mathrm{u}, \mathrm{m}), 110.8(\mathrm{u}), 95.8(\mathrm{u}), 64.7(\mathrm{u}), 59.5(\mathrm{dn}), 32.1(\mathrm{u}) ;{ }^{19} \mathrm{~F}$ NMR

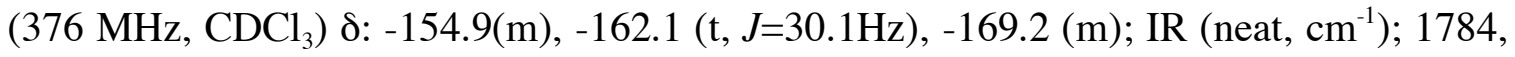
1521, 1248, 1116, 1028, 1011; HRMS (ESI+) m/z: [M+Na] calcd for $\mathrm{C}_{19} \mathrm{H}_{6} \mathrm{~F}_{10} \mathrm{O}_{5}$ : 526.9953; found: 526.9953 .

bis-Pentafluorophenyl 2-trityloxymethyl-cycloprop-2-ene-1,1-bis-carboxylic ester $(4 \mathrm{~g})$

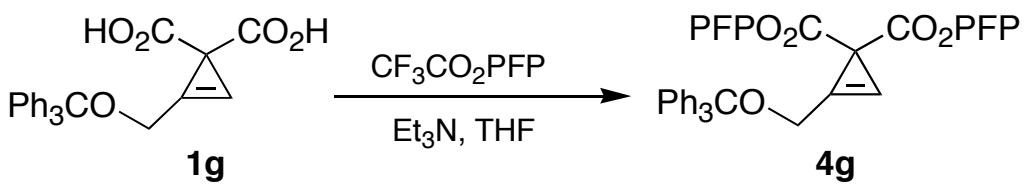


The general procedure for making bis-pentafluorophenyl esters ester with $\mathbf{1 g}$ (80 $\mathrm{mg}, 0.2$ $\mathrm{mmol}$ ), pentafluorophenyl trifluoroacetate (114 mg, $0.4 \mathrm{mmol}), \mathrm{Et}_{3} \mathrm{~N}$ (48.5 mg, 0.48 mmol), and THF (2 mL) gave $102 \mathrm{mg}(70 \%)$ of the title compound as semisolid. A similar reaction gave $102 \mathrm{mg}(70 \%)$ of the title compound. The purity of the compound was judged to be $95 \%$ by ${ }^{1} \mathrm{H}$ NMR. Minor impurities were detected in the ${ }^{1} \mathrm{H}$ NMR at 7.18 ppm, 6.64 ppm, 6.44 ppm, 5.49 ppm, 5.20 ppm, 4.11 ppm, 3.79 ppm, 3.52 ppm, 1.88 ppm, $1.34 \mathrm{ppm}, 1.29 \mathrm{ppm}, 0.90 \mathrm{ppm}$. Because of the sensitivity of the compound at silica gel, the compound was used in the next step without further purification. ${ }^{1} \mathrm{H}$ NMR (400

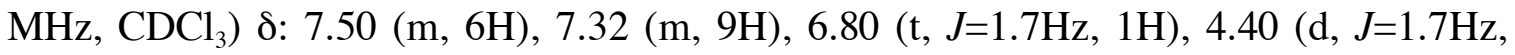
$2 \mathrm{H}) ;{ }^{13} \mathrm{C}$ NMR (100 MHz, $\mathrm{CDCl}_{3}$ ) $\delta: 165.7(\mathrm{u}), 143.3(\mathrm{u}), 141.5(\mathrm{u}, \mathrm{dm}, J=250 \mathrm{~Hz}), 140.1$ (u, dm, $J=260 H z), 138.3$ (u, dm, $J=240 H z), 128.9$ (dn), 128.5 (dn), 127.9 (dn), 125.4 (u,

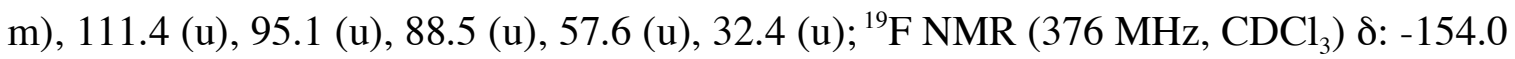
(m), -161.7 (t, $J=33.8 H z),-168.7(\mathrm{~m})$; IR (neat, $\left.\mathrm{cm}^{-1}\right): 1780,1517,995,907,765,704$; HRMS (ESI+) m/z: [M+Na] calcd for $\mathrm{C}_{37} \mathrm{H}_{18} \mathrm{~F}_{10} \mathrm{O}_{5}$ : 755.0892; found: 755.0868 .

\section{General procedure for the desymmetrization of bis-pentafluorophenyl cyclopropene}

\section{bis-carboxylic esters}

A solution of $(S)$-4-phenyloxazolidinone $(163 \mathrm{mg}, 1.0 \mathrm{mmol})$ in $9.5 \mathrm{~mL}$ THF was cooled in a bath at $-78^{\circ} \mathrm{C}$ (dry ice/acetone). To this solution was added $n$-BuLi (1.0 mmol, 0.5 $\mathrm{mL}$ of a $2.0 \mathrm{M}$ pentane solution) via syringe, and the mixture was stirred for $30 \mathrm{~min}$ at $-78^{\circ} \mathrm{C}$. A separate $100 \mathrm{~mL}$ round bottomed flask containing the appropriate bispentafluorophenyl cycloprop-2-ene-1,1-bis-carboxylic ester $(1.0 \mathrm{mmol})$ and $\mathrm{CH}_{2} \mathrm{Cl}_{2}(30$ $\mathrm{mL}$ ) was cooled in a bath at $-78^{\circ} \mathrm{C}$ (dry ice/acetone bath). To this magnetically stirred 
solution was injected via syringe the solution of the lithiated oxazolidione (prepared above). The mixture was allowed to stir for $20 \mathrm{~min}$ at $-78^{\circ} \mathrm{C}$, at which point the cold bath was removed and the temperature allowed to rise to rt. The solvents were removed under reduced pressure, and the residue was partitioned between ether $(50 \mathrm{~mL})$ and water $(50$ $\mathrm{mL})$. The organic layer was washed with water $(2 \times 20 \mathrm{~mL})$, dried over $\mathrm{Na}_{2} \mathrm{SO}_{4}$, filtered, and concentrated. The residue was chromatographed on silica gel (10-30\% ether in hexanes) to provide the products. The diastereomeric ratio of the crude mixture was determined by HPLC analysis using a normal phase silica column.

\section{Pentafluorophenyl 2-butyl-1-(2-oxo-4S-phenyl-oxazolidine-3-carbonyl)-cycloprop-2- ene carboxylic ester [5a(major) \& 5a(minor)]}

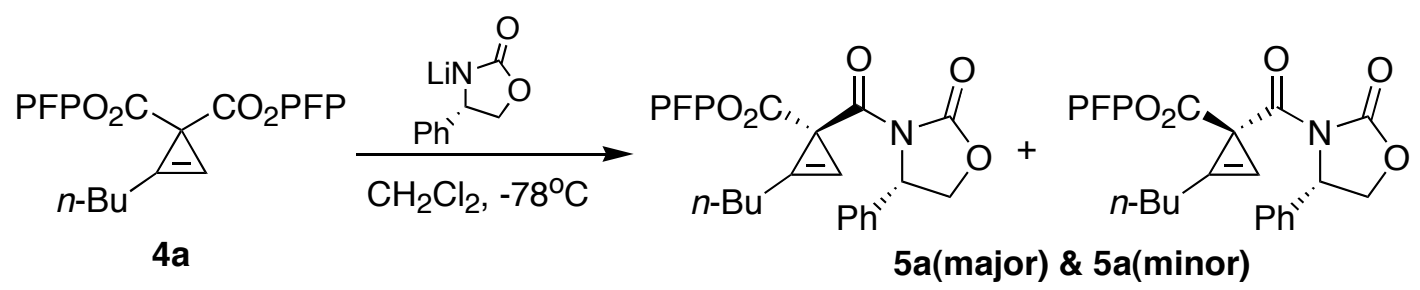

The general procedure for desymmetrization with $4 \mathbf{a}(1.28 \mathrm{~g}, 2.47 \mathrm{mmol})$ gave $1.10 \mathrm{~g}$ (90\%) of 5a(major) and $0.06 \mathrm{~g}(5 \%)$ of $\mathbf{5 a ( m i n o r ) . ~ B o t h ~ d i a s t e r e o m e r s ~ a r e ~ s e m i s o l i d s . ~ A ~}$ similar reaction starting from $3.06 \mathrm{~g} \mathbf{4 a}$ gave $2.53 \mathrm{~g}(88 \%)$ of $\mathbf{5 a}$ (major) and $120 \mathrm{mg}$ (4\%) of of $\mathbf{5 a}$ (minor). The diastereomeric ratio of the crude mixture was determined to be $96: 4$ by HPLC (3\% isopropanol in hexane at $1 \mathrm{~mL} / \mathrm{min}$ ).

5a(major). ${ }^{1} \mathrm{H}$ NMR (400 MHz, $\left.\mathrm{CDCl}_{3}\right) \delta: 7.35$ (m, 5H), $6.58(\mathrm{t}, J=1.5 \mathrm{~Hz}, 1 \mathrm{H}), 5.44$ (dd, $J=8.9 \mathrm{~Hz}, 5.3 \mathrm{~Hz}, 1 \mathrm{H}), 4.76(\operatorname{app} \mathrm{t}, J=8.9 \mathrm{~Hz}, 1 \mathrm{H}), 4.30(\mathrm{dd}, J=8.9 \mathrm{~Hz}, 5.3 \mathrm{~Hz}, 1 \mathrm{H}), 2.65(\mathrm{~m}$, 2H), $1.66(\mathrm{~m}, 2 \mathrm{H}), 1.40(\mathrm{~m}, 2 \mathrm{H}), 0.92(\mathrm{t}, J=7.3 \mathrm{~Hz}, 3 \mathrm{H}) ;{ }^{13} \mathrm{C} \mathrm{NMR}\left(100 \mathrm{MHz}, \mathrm{CDCl}_{3}\right) \delta$ : $169.7(\mathrm{u}), 168.2(\mathrm{u}), 154.3(\mathrm{u}), 141.6\left(\mathrm{u}, \mathrm{dm}, J_{\mathrm{FC}}=251 \mathrm{~Hz}\right), 139.9\left(\mathrm{u}, \mathrm{dm}, J_{\mathrm{FC}}=260 \mathrm{~Hz}\right)$, 
$138.5(\mathrm{u}), 138.2\left(\mathrm{u}, \mathrm{dm}, J_{\mathrm{FC}}=260 \mathrm{~Hz}\right), 129.6(\mathrm{dn}), 129.2(\mathrm{dn}), 126.5(\mathrm{dn}), 125.4(\mathrm{u}, \mathrm{m})$, $116.6(u), 94.4(u), 70.9(u), 58.3(d n), 35.6(u), 28.9(u), 24.5(u), 22.6(u), 14.1(d n) ;{ }^{19} \mathrm{~F}$ NMR (376 MHz, $\left.\mathrm{CDCl}_{3}\right)$ : -153.4 (m), -162.8 (t, J=33.8Hz), -169.1(m ); IR (neat, $\left.\mathrm{cm}^{-1}\right)$ : 2980, 2869, 1787, 1757, 1706, 1521, 1385, 1330, 1215, 1143, 1030, 998, 912, 743; $[\alpha]_{\mathrm{D}}^{25}=-11.0^{\circ}\left(\mathrm{c} 1.00, \mathrm{CH}_{2} \mathrm{Cl}_{2}\right)$. HRMS (ESI+) m/z: [M+Na] calcd for $\mathrm{C}_{24} \mathrm{H}_{18} \mathrm{~F}_{5} \mathrm{NO}_{5}$ : 518.1003; found: 518.1083 .

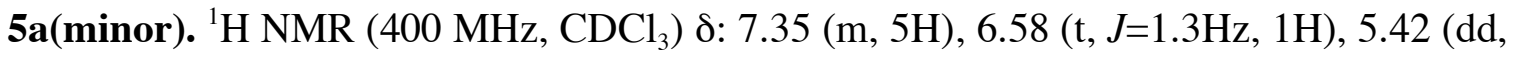
$J=8.9 \mathrm{~Hz}, 4.8 \mathrm{~Hz}, 1 \mathrm{H}), 4.75(\operatorname{app~t}, J=8.9 \mathrm{~Hz}, 1 \mathrm{H}), 4.28(\mathrm{dd}, J=8.9 \mathrm{~Hz}, 4.8 \mathrm{~Hz}, 1 \mathrm{H}), 2.69$ (m, 2H), $1.63(\mathrm{~m}, 2 \mathrm{H}), 1.40(\mathrm{~m}, 2 \mathrm{H}), 0.90(\mathrm{t}, J=7.3 \mathrm{~Hz}, 3 \mathrm{H}) ;{ }^{13} \mathrm{C} \mathrm{NMR}\left(100 \mathrm{MHz}, \mathrm{CDCl}_{3}\right) \delta$ : $169.6(\mathrm{u}), 168.2(\mathrm{u}), 154.3(\mathrm{u}), 141.6\left(\mathrm{u}, \mathrm{dm}, J_{\mathrm{FC}}=250 \mathrm{~Hz}\right), 139.9\left(\mathrm{u}, \mathrm{dm}, J_{\mathrm{FC}}=230 \mathrm{~Hz}\right)$, $138.8(\mathrm{u}), 138.2\left(\mathrm{u}, \mathrm{dm}, J_{\mathrm{FC}}=240 \mathrm{~Hz}\right), 129.6(\mathrm{dn}), 129.1(\mathrm{dn}), 126.4(\mathrm{dn}), 125.5(\mathrm{u}, \mathrm{m})$, $116.8(\mathrm{u}), 95.0(\mathrm{u}), 70.9(\mathrm{u}), 58.1(\mathrm{dn}), 35.2(\mathrm{u}), 28.8(\mathrm{u}), 24.4(\mathrm{u}), 22.6(\mathrm{u}), 14.0(\mathrm{dn}) ;{ }^{19} \mathrm{~F}$

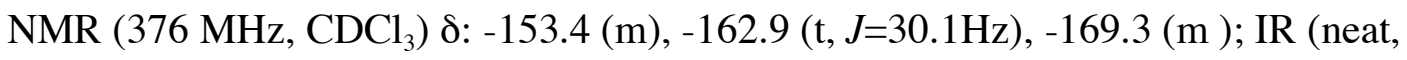
$\left.\mathrm{cm}^{-1}\right): 1780,1696,1518,1385,1327,1200,1028,994,908,725 ;[\alpha]_{\mathrm{D}}{ }^{25}=-28.0^{\circ}(\mathrm{c} 1.00$, $\mathrm{CH}_{2} \mathrm{Cl}_{2}$ ); HRMS (ESI+) m/z: [M+Na] calcd for $\mathrm{C}_{24} \mathrm{H}_{18} \mathrm{~F}_{5} \mathrm{NO}_{5}$ : 518.1003; found: 518.1027.

\section{Pentafluorophenyl 1-(2-oxo-4S-phenyl-oxazolidine-3-carbonyl)-2-phenyl-cycloprop-} 2-ene carboxylic ester [5b(Rcy, Sox $) \& 5 b(S c y, S o x)]$

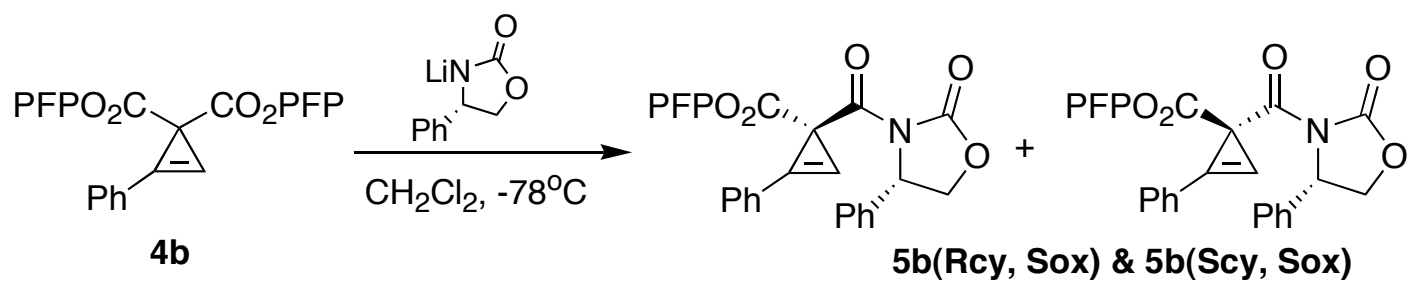


The general procedure for desymmetrization with $\mathbf{4 b}(210 \mathrm{mg}, 0.39 \mathrm{mmol})$ gave $175 \mathrm{mg}$ $(87.5 \%)$ of $\mathbf{5 b}(\boldsymbol{R} \mathbf{c y}, \boldsymbol{S o x})$ as white solid. A small amount of $\mathbf{5 b}(\boldsymbol{S c y}, \boldsymbol{S o x})(<1.0 \mathrm{mg})$ was also isolated. A similar reaction gave $170 \mathrm{mg}(85 \%)$ of $\mathbf{5 b}(\boldsymbol{R c y}, \mathbf{S o x})$. The diastereomeric ratio of the crude mixture was determined to be $99.5: 0.5$ by HPLC (3\% isopropanol in hexane at $1 \mathrm{~mL} / \mathrm{min})$. The absolute configuration was proven by x-ray crystallography.

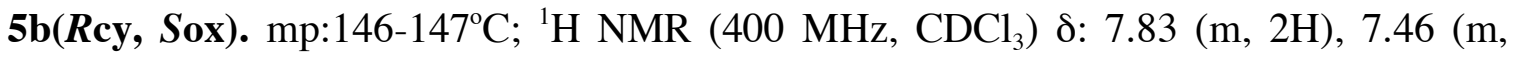
$3 \mathrm{H}), 7.38(\mathrm{~m}, 5 \mathrm{H}), 7.06(\mathrm{~s}, 1 \mathrm{H}), 5.46(\mathrm{dd}, J=8.9,4.9 \mathrm{~Hz}, 1 \mathrm{H}), 4.79(\operatorname{app~t}, J=9.0 \mathrm{~Hz}, 1 \mathrm{H})$, $4.34(\mathrm{dd}, J=9.0,4.9 \mathrm{~Hz}, 1 \mathrm{H}) ;{ }^{13} \mathrm{C}$ NMR $\left(100 \mathrm{MHz}, \mathrm{CDCl}_{3}\right) \delta: 168.8(\mathrm{u}), 167.7(\mathrm{u}), 154.5$ $(\mathrm{u}), 141.2\left(\mathrm{u}, \mathrm{dm}, J_{\mathrm{FC}}=260 \mathrm{~Hz}\right), 139.5\left(\mathrm{u}, \mathrm{dm}, J_{\mathrm{FC}}=255 \mathrm{~Hz}\right), 138.6(\mathrm{u}), 138.2(\mathrm{u}, \mathrm{dm}$, $\left.J_{\mathrm{FC}}=240 \mathrm{~Hz}\right), 131.5(\mathrm{dn}), 131.1(\mathrm{dn}), 129.7(\mathrm{dn}), 129.4(\mathrm{dn}), 129.3(\mathrm{dn}), 126.4(\mathrm{dn}), 125.4$ (u, m), $124.0(\mathrm{u}), 113.4(\mathrm{u}), 95.7(\mathrm{u}), 71.1(\mathrm{u}), 58.4(\mathrm{dn}), 36.3(\mathrm{u}) ;{ }^{19} \mathrm{~F}$ NMR $(376 \mathrm{MHz}$, $\left.\mathrm{CDCl}_{3}\right) \delta:-153.3(\mathrm{~m}),-162.5(\mathrm{t}, J=33.8 \mathrm{~Hz}),-169.1(\mathrm{~m}) ; \mathrm{IR}$ (neat, $\left.\mathrm{cm}^{-1}\right): 1789,1757$, $1694,1517,1392,1334,1302,1213,1119,1062,1018,990,958,916,890,768,734$, 724, 699, 687; $[\alpha]_{\mathrm{D}}{ }^{25}=+13.2^{\circ}(\mathrm{c} 1.00, \mathrm{THF}) ; \mathrm{HRMS}(\mathrm{ESI}+) \mathrm{m} / \mathrm{z}:[\mathrm{M}+\mathrm{Na}]$ calcd for $\mathrm{C}_{26} \mathrm{H}_{14} \mathrm{~F}_{5} \mathrm{NO}_{5}$ : 538.0690; found: 538.0671 .

$\mathbf{5 b}(\mathbf{S c y}, \mathbf{S o x}) .(\sim 70 \%$ purity. Because the diastereoselectivity of the reaction was so high (99.5:0.5), it was difficult to obtain the minor diastereomer in higher purity.) ${ }^{1} \mathrm{H}$ NMR

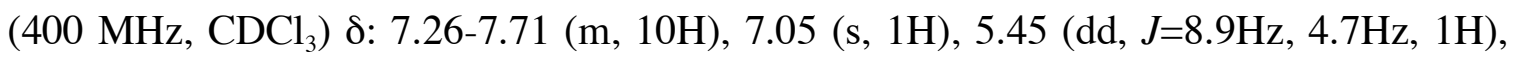
$4.78(\operatorname{app~t}, J=8.9 \mathrm{~Hz}, 1 \mathrm{H}), 4.32(\mathrm{dd}, J=8.9 \mathrm{~Hz}, 4.7 \mathrm{~Hz}, 1 \mathrm{H}) ;{ }^{19} \mathrm{~F}$ NMR $\left(376 \mathrm{MHz}, \mathrm{CDCl}_{3}\right)$

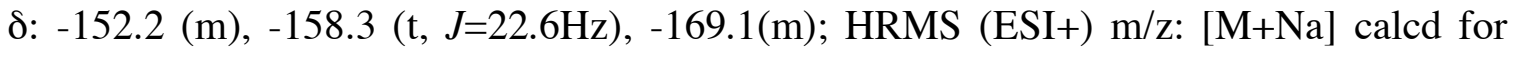
$\mathrm{C}_{26} \mathrm{H}_{14} \mathrm{~F}_{5} \mathrm{NO}_{5}:$ 538.0690; found: 538.0675 . 


\section{Pentafluorophenyl 2-isopropyl-1-(2-oxo-4S-phenyl-oxazolidine-3-carbonyl)- cycloprop-2-ene carboxylic ester [5c(major) \& 5c(minor)]}

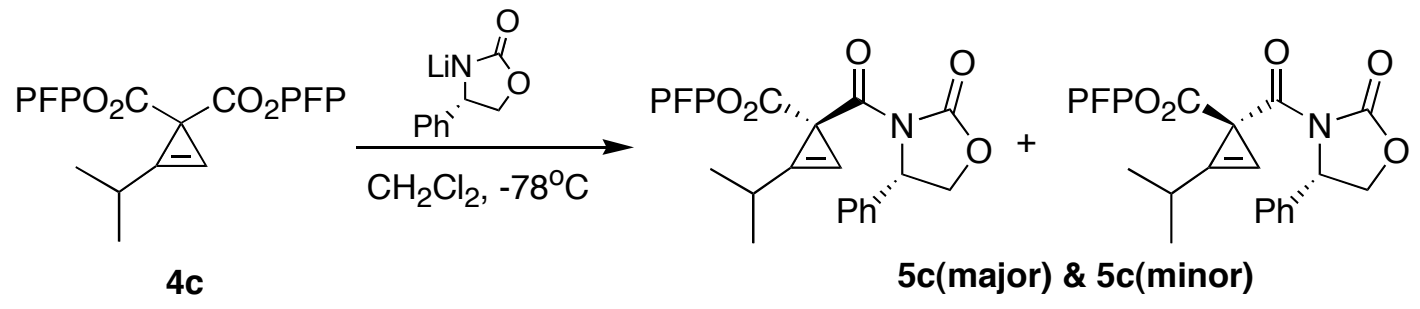

The general procedure for desymmetrization with $4 \mathbf{c}(182 \mathrm{mg}, 0.36 \mathrm{mmol})$ gave $155 \mathrm{mg}$ (90\%) of the major diastereomer $\mathbf{5 c}$ (major) as a semisolid. A similar reaction starting from $197 \mathrm{mg} \mathrm{4c}$ gave $170 \mathrm{mg}(91 \%)$ of $\mathbf{5 c}$ (major). The diastereomeric ratio of the crude mixture was determined to be 99:1 by HPLC (3\% isopropanol in hexane at $1 \mathrm{~mL} / \mathrm{min}$ ).

To prepare an analytical sample of the minor diastereomer, the desymmetrizaition was carried out at $\mathrm{rt}$ (instead of $-78^{\circ} \mathrm{C}$ ). Thus, the procedure was similar to the general procedure for desymmetrization except that the solution of lithiated oxazolidione $(0.32$ $\mathrm{mmol}$ in $2 \mathrm{~mL}$ THF) was added to the solution of $4 \mathbf{c}(162 \mathrm{mg}, 0.32 \mathrm{mmol})$ in THF $(3 \mathrm{~mL})$ at rt. This protocol gave $130 \mathrm{mg}(84 \%)$ of $\mathbf{5 c}$ (major) and $10 \mathrm{mg}(6 \%)$ of $\mathbf{5 c}$ (minor). The diastereomeric ratio of the crude mixture was determined to be $92: 8$ by HPLC (3\% isopropanol in hexane at $1 \mathrm{~mL} / \mathrm{min}$ ).

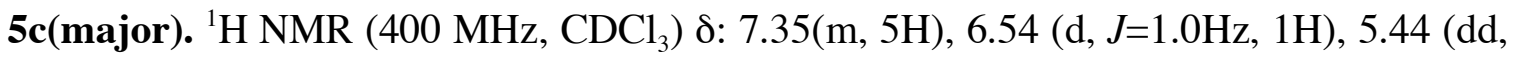
$J=8.8 \mathrm{~Hz}, 5.2 \mathrm{~Hz}, 1 \mathrm{H}), 4.75(\operatorname{app} \mathrm{t}, J=9.0 \mathrm{~Hz}, 1 \mathrm{H}), 4.29(\mathrm{dd}, J=8.9 \mathrm{~Hz}, 5.2 \mathrm{~Hz}, 1 \mathrm{H}), 3.00(\mathrm{~m}$, $1 \mathrm{H}), 1.28(\mathrm{~d}, J=4.8 \mathrm{~Hz}, 3 \mathrm{H}), 1.26(\mathrm{~d}, J=4.7 \mathrm{~Hz}, 3 \mathrm{H}) ;{ }^{13} \mathrm{C} \mathrm{NMR}\left(100 \mathrm{MHz}, \mathrm{CDCl}_{3}\right) \delta: 169.7$ $(\mathrm{u}), 168.2(\mathrm{u}), 154.3(\mathrm{u}), 141.6\left(\mathrm{u}, \mathrm{dm}, J_{\mathrm{FC}}=250 \mathrm{~Hz}\right), 139.9\left(\mathrm{u}, \mathrm{dm}, J_{\mathrm{FC}}=250 \mathrm{~Hz}\right), 138.6(\mathrm{u})$, $138.3\left(\mathrm{u}, \mathrm{dm}, J_{\mathrm{FC}}=250 \mathrm{~Hz}\right), 129.6(\mathrm{dn}), 129.2(\mathrm{dn}), 126.4(\mathrm{dn}), 125.4(\mathrm{u}, \mathrm{m}), 120.8(\mathrm{u})$, $93.4(\mathrm{u}), 71.0(\mathrm{u}), 58.4(\mathrm{dn}), 36.1(\mathrm{u}), 26.3(\mathrm{dn}), 20.5(\mathrm{dn}), 20.2(\mathrm{dn}) ;{ }^{19} \mathrm{~F}$ NMR $(376 \mathrm{MHz}$, 
$\left.\mathrm{CDCl}_{3}\right) \delta:-153.3(\mathrm{~m}),-162.9(\mathrm{t}, J=30.1 \mathrm{~Hz}),-169.4(\mathrm{~m})$; IR (neat, $\left.\mathrm{cm}^{-1}\right): 1787,1696,1518$, $1387,1326,1199,1100,1031,995,735,701 ;[\alpha]_{\mathrm{D}}{ }^{25}=-12.1^{\circ}\left(\mathrm{c} 1.00, \mathrm{CH}_{2} \mathrm{Cl}_{2}\right) ; \mathrm{HRMS}$ (CI) $\mathrm{m} / \mathrm{z}:[\mathrm{M}+\mathrm{H}]$ calcd for $\mathrm{C}_{23} \mathrm{H}_{16} \mathrm{~F}_{5} \mathrm{NO}_{5}$ : 482.1027; found: 482.1034 .

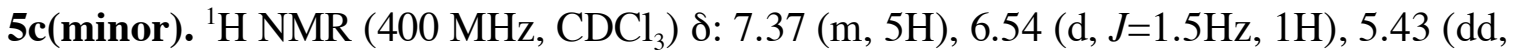
$J=8.9 \mathrm{~Hz}, 4.8 \mathrm{~Hz}, 1 \mathrm{H}), 4.76(\operatorname{app~t}, J=8.9 \mathrm{~Hz}, 1 \mathrm{H}), 4.30(\mathrm{dd}, J=8.9 \mathrm{~Hz}, 4.8 \mathrm{~Hz}, 1 \mathrm{H}), 3.03(\mathrm{~m}$, 1H), $1.26(\mathrm{~d}, \mathrm{~J}=6.9 \mathrm{~Hz}, 3 \mathrm{H}), 1.22(\mathrm{~d}, \mathrm{~J}=7.0 \mathrm{~Hz}, 3 \mathrm{H}) ;{ }^{13} \mathrm{C} \mathrm{NMR}\left(100 \mathrm{MHz}, \mathrm{CDCl}_{3}\right) \delta: 169.1$, $167.8,153.8,141.2\left(\mathrm{dm}, J_{\mathrm{FC}}=250 \mathrm{~Hz}\right), 139.5\left(\mathrm{dm}, J_{\mathrm{FC}}=250 \mathrm{~Hz}\right), 138.3,137.8(\mathrm{dm}$, $\left.J_{\mathrm{FC}}=240 \mathrm{~Hz}\right), 129.2,128.8,125.9,121.0,93.7,70.5,57.8,35.3,29.7,25.6,20.2,19.8$; (The carbon attached to the oxygen of the pentafluorophenyl group was not observed.)

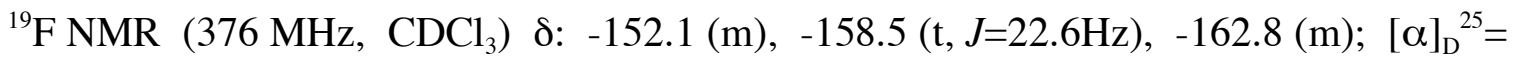
$-17.6^{\circ}$ (c 0.5, THF); IR $\left(\mathrm{CH}_{2} \mathrm{Cl}_{2}, \mathrm{~cm}^{-1}\right): 3055,2987,1787,1703,1522,1422,1387,1328$, 1266, 1191, 1069, 1036, 997, 896, 739, 705; HRMS (CI) m/z: [M+H] calcd for $\mathrm{C}_{23} \mathrm{H}_{16} \mathrm{~F}_{5} \mathrm{NO}_{5}$ : 482.1027; found: 482.1013 .

\section{Pentafluorophenyl 2-(4-fluorophenyl)-1-(2-oxo-4S-phenyl-oxazolidine-3-carbonyl)-} cycloprop-2-ene carboxylic ester [5d(major) \& 5d(minor)]

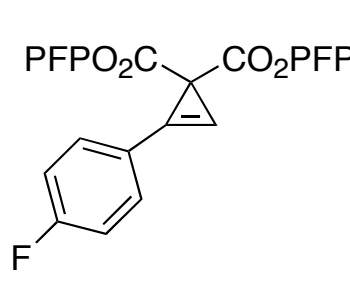

4d

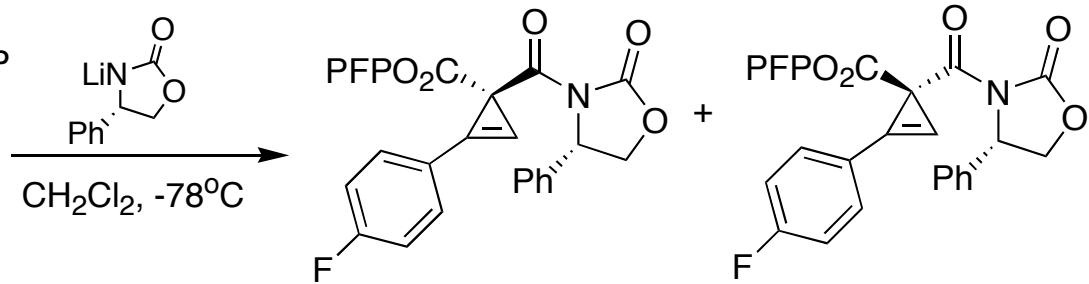

$5 d($ major $) \& 5 d($ minor $)$

The procedure was identical to the procedure for desymmetrization, except that a solution of lithiated oxazolidinone (1.59 mmol in $10 \mathrm{~mL}$ THF) and a solution of $\mathbf{4 d}(880 \mathrm{mg}, 1.59$ mmol) in $\mathrm{CH}_{2} \mathrm{Cl}_{2}(100 \mathrm{~mL})$ was used. The protocol gave $794 \mathrm{mg}$ (94\%) of $\mathbf{5 d ( m a j o r ) . ~ A ~}$ 
similar reaction starting from $230 \mathrm{mg}$ of $\mathbf{4 d}$ gave $200 \mathrm{mg}$ (90\%) of $\mathbf{5 d}$ (major). The diastereomeric ratio of the crude mixture was determined to be 99: 1 by HPLC analysis (3\% isopropanol in hexane at $1 \mathrm{~mL} / \mathrm{min}$ ).

To prepare an analytical sample of the minor diastereomer, the desymmetrizaition was carried out at rt (instead of $-78^{\circ} \mathrm{C}$ ). Thus, the procedure was similar to the general procedure for desymmetrization except that the solution of lithiated oxazolidione $(0.27$ mmol in $2 \mathrm{~mL}$ THF) was added to the solution of $4 \mathbf{d}(152 \mathrm{mg}, 0.27 \mathrm{mmol})$ in THF (3 $\mathrm{mL}$ ) at rt. This protocol gave $100 \mathrm{mg}(70 \%)$ of $\mathbf{5 d}$ (major) and $12 \mathrm{mg}(8 \%)$ of $\mathbf{5 d}$ (minor). The diastereomeric ratio of the crude mixture was determined to be 90:10 by HPLC analysis (3\% isopropanol in hexane at $1 \mathrm{~mL} / \mathrm{min}$ ).

5d(major). Pale yellow solid; mp: 63-64 ${ }^{\circ} \mathrm{C} ;{ }^{1} \mathrm{H}$ NMR (400 MHz, $\left.\mathrm{CDCl}_{3}\right)$ ): $7.88(\mathrm{~m}, 2 \mathrm{H})$, $7.42(\mathrm{~m}, 5 \mathrm{H}), 7.17(\mathrm{~m}, 2 \mathrm{H}), 7.08(\mathrm{~s}, 1 \mathrm{H}), 5.50(\mathrm{dd}, J=8.9 \mathrm{~Hz}, 5.0 \mathrm{~Hz}, 1 \mathrm{H}), 4.83($ app t, $J=8.9 \mathrm{~Hz}, 1 \mathrm{H}), 4.38(\mathrm{dd}, J=8.9 \mathrm{~Hz}, 5.0 \mathrm{~Hz}, 1 \mathrm{H}) ;{ }^{13} \mathrm{C} \mathrm{NMR}\left(100 \mathrm{MHz}, \mathrm{CDCl}_{3}\right) \delta: 168.7(\mathrm{u})$, $167.6(\mathrm{u}), 164.7\left(\mathrm{u}, \mathrm{d}, J_{\mathrm{FC}}=252 \mathrm{~Hz}\right), 154.7(\mathrm{u}), 141.6\left(\mathrm{u}, \mathrm{dm}, J_{\mathrm{FC}}=240 \mathrm{~Hz}\right), 139.9(\mathrm{u}, \mathrm{dm}$, $\left.J_{\mathrm{FC}}=250 \mathrm{~Hz}\right), 138.6(\mathrm{u}), 138.3\left(\mathrm{u}, \mathrm{dm}, J_{\mathrm{FC}}=260 \mathrm{~Hz}\right), 133.3\left(\mathrm{dn}, \mathrm{d}, J_{\mathrm{FC}}=8.9 \mathrm{~Hz}\right), 129.7(\mathrm{dn})$, $129.3(\mathrm{dn}), 126.5(\mathrm{dn}), 125.4(\mathrm{u}, \mathrm{m}), 120.3\left(\mathrm{u}, \mathrm{d}, J_{\mathrm{FC}}=3.2 \mathrm{~Hz}\right), 116.8\left(\mathrm{dn}, \mathrm{d}, J_{\mathrm{FC}}=22.1 \mathrm{~Hz}\right)$, $112.4(\mathrm{u}), 95.3\left(\mathrm{u}, \mathrm{d}, J_{\mathrm{FC}}=2.1 \mathrm{~Hz}\right), 71.2(\mathrm{u}), 58.5(\mathrm{dn}), 36.4(\mathrm{u}) ;{ }^{19} \mathrm{~F}$ NMR $(376 \mathrm{MHz}$, $\left.\mathrm{CDCl}_{3}\right) \delta:-86.5(\mathrm{~m}),-153.5(\mathrm{~m}),-162.3(\mathrm{t}, J=33.8 \mathrm{~Hz}),-169.1(\mathrm{~m})$; IR (neat, $\left.\mathrm{cm}^{-1}\right): 1782$, $1696,1600,1519,1504,1387,1329,1300,1203,1157,1107,1031,995,908,841,762$, 728, 649; $[\alpha]_{D}^{25}=+23.5^{\circ}\left(\right.$ c $\left.1.00, \mathrm{CH}_{2} \mathrm{Cl}_{2}\right)$. HRMS (ESI+) $\mathrm{m} / \mathrm{z}$ : $[\mathrm{M}+\mathrm{Na}]$ calcd for $\mathrm{C}_{26} \mathrm{H}_{13} \mathrm{~F}_{6} \mathrm{NO}_{5}$ : 556.0596; found: 556.0620. 
5d(minor). (The purity of the compound was judged to be $\sim 70 \%$ by ${ }^{1} \mathrm{H}$ NMR. Further purification was not pursued for the minor diastereomer.) ${ }^{1} \mathrm{H} \mathrm{NMR}\left(400 \mathrm{MHz}, \mathrm{CDCl}_{3}\right) \delta$ : $7.40(\mathrm{~m}, 7 \mathrm{H}), 7.05(\mathrm{~m}, 2 \mathrm{H}), 6.85(\mathrm{~s}, 1 \mathrm{H}), 5.60(\mathrm{dd}, J=8.8 \mathrm{~Hz}, 7.1 \mathrm{~Hz}, 1 \mathrm{H}), 4.77$ (app t, $J=8.8 \mathrm{~Hz}, 1 \mathrm{H}), 4.29(\mathrm{dd}, J=8.8 \mathrm{~Hz}, 7.1 \mathrm{~Hz}, 1 \mathrm{H}) ;{ }^{19} \mathrm{~F}$ NMR $\left(376 \mathrm{MHz}, \mathrm{CDCl}_{3}\right) \delta:-112.9$ (m), -153.6 (m), -157.9 (t, J=22.6Hz), -161.5 (m); HRMS (CI) m/z: [M+] calcd for $\mathrm{C}_{26} \mathrm{H}_{13} \mathrm{~F}_{6} \mathrm{NO}_{5}$ : 533.0680; found: 533.0688.

\section{Pentafluorophenyl 2-isobutyl-1-(2-oxo-4-phenyl-oxazolidine-3-carbonyl)-cycloprop-} 2-ene carboxylic ester [5e(major) \& 5e(minor)]

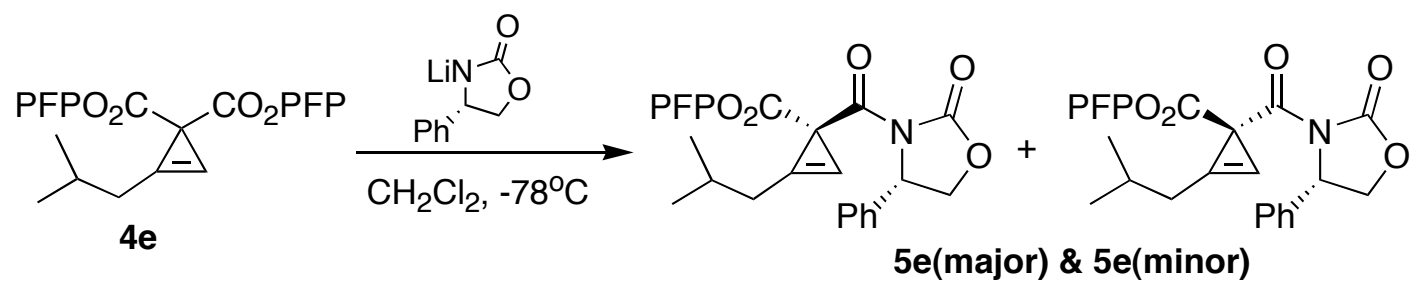

The general procedure for desymmetrization with 4 e $(196 \mathrm{mg}, 0.38 \mathrm{mmol})$ gave $150 \mathrm{mg}$ $(80 \%)$ of $\mathbf{5 e}$ (major) and $8 \mathrm{mg}(4 \%)$ of $\mathbf{5 e}(\mathbf{m i n o r})$. Both diastereomers are semisolids. A similar reaction starting from $201 \mathrm{mg}$ of $\mathbf{4 e}$ gave $158 \mathrm{mg}(82 \%)$ of $\mathbf{5 e}(\mathbf{m a j o r})$ and $10 \mathrm{mg}$ $(5 \%)$ of $\mathbf{5 e}$ (minor). The diastereomeric ratio of the crude mixture was determined to be 95:5 by HPLC (3\% isopropanol in hexane at $1 \mathrm{~mL} / \mathrm{min})$.

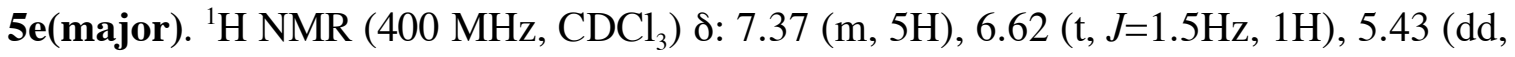
$J=9.0 \mathrm{~Hz}, 5.4 \mathrm{~Hz}, 1 \mathrm{H}), 4.75(\operatorname{app~t}, J=9.0 \mathrm{~Hz}, 1 \mathrm{H}), 4.28(\mathrm{dd}, J=9.0 \mathrm{~Hz}, 5.4 \mathrm{~Hz}, 1 \mathrm{H}), 2.63$ (ddd, $J=16.7 \mathrm{~Hz}, 6.7 \mathrm{~Hz}, 1.4 \mathrm{~Hz}, 1 \mathrm{H}), 2.50(\mathrm{ddd}, J=16.7 \mathrm{~Hz}, 6.8 \mathrm{~Hz}, 1.5 \mathrm{~Hz}, 1 \mathrm{H}), 2.05$ (m, $1 \mathrm{H}), 1.01(\mathrm{~d}, J=2.5 \mathrm{~Hz}, 3 \mathrm{H}), 1.00(\mathrm{~d}, J=2.5 \mathrm{~Hz}, 3 \mathrm{H}) ;{ }^{13} \mathrm{C} \mathrm{NMR}\left(100 \mathrm{MHz}, \mathrm{CDCl}_{3}\right) \delta: 169.7$ $(\mathrm{u}), 168.2(\mathrm{u}), 154.3(\mathrm{u}), 141.5\left(\mathrm{u}, \mathrm{dm}, J_{\mathrm{FC}}=250 \mathrm{~Hz}\right), 140.0\left(\mathrm{u}, \mathrm{dm}, J_{\mathrm{FC}}=250 \mathrm{~Hz}\right), 138.5(\mathrm{u})$, $138.3\left(\mathrm{u}, \mathrm{dm}, J_{\mathrm{FC}}=250 \mathrm{~Hz}\right), 129.6(\mathrm{dn}), 129.2(\mathrm{dn}), 126.5(\mathrm{dn}), 125.5(\mathrm{u}, \mathrm{m}), 115.8(\mathrm{u})$, 
$94.9(\mathrm{u}), 70.9(\mathrm{u}), 58.3(\mathrm{dn}), 35.3(\mathrm{u}), 33.6(\mathrm{u}), 27.1(\mathrm{dn}), 22.65(\mathrm{dn}), 22.63(\mathrm{dn}),{ }^{19} \mathrm{~F}$ NMR

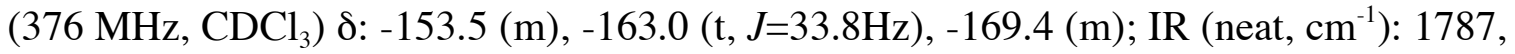
$1696,1518,1386,1326,1212,1148,1100,1029,997,908,763,730 ;[\alpha]_{\mathrm{D}}^{25}=$

$-17.5^{\circ}\left(\mathrm{c} 1.00, \mathrm{CH}_{2} \mathrm{Cl}_{2}\right) ;$ HRMS (ESI+) m/z: [M+Na] calcd for $\mathrm{C}_{24} \mathrm{H}_{18} \mathrm{~F}_{5} \mathrm{NO}_{5}: 518.1003$; found: 518.1026 .

5e(minor). ${ }^{1} \mathrm{H}$ NMR $\left(400 \mathrm{MHz}, \mathrm{CDCl}_{3}\right) \delta: 7.38(\mathrm{~m}, 5 \mathrm{H}), 6.60(\mathrm{t}, J=1.5 \mathrm{~Hz}, 1 \mathrm{H}), 5.43(\mathrm{dd}$, $J=9.0 \mathrm{~Hz}, 5.4 \mathrm{~Hz}, 1 \mathrm{H}), 4.77(\operatorname{app} \mathrm{t}, J=9.0 \mathrm{~Hz}, 1 \mathrm{H}), 4.31(\mathrm{dd}, J=9.0 \mathrm{~Hz}, 5.4 \mathrm{~Hz}, 1 \mathrm{H}), 2.56(\mathrm{~m}$, 2H), $2.01(\mathrm{~m}, 1 \mathrm{H}), 0.98(\mathrm{~d}, J=6.7 \mathrm{~Hz}, 3 \mathrm{H}), 0.96(\mathrm{~d}, J=6.6 \mathrm{~Hz}, 3 \mathrm{H}) ;{ }^{13} \mathrm{C}$ NMR $(100 \mathrm{MHz}$,

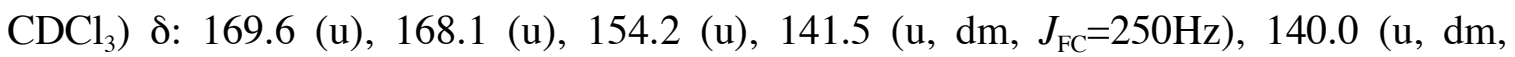
$\left.J_{\mathrm{FC}}=250 \mathrm{~Hz}\right), 138.5(\mathrm{u}), 138.3\left(\mathrm{u}, \mathrm{dm}, J_{\mathrm{FC}}=250 \mathrm{~Hz}\right), 129.6(\mathrm{dn}), 129.2(\mathrm{dn}), 126.4(\mathrm{dn})$, $116.0(u), 95.5(u), 70.9(u), 58.2(d n), 35.0(u), 33.6(u), 27.1(d n), 22.7(d n)$; (The carbon attached to the oxygen of the pentafluorophenyl group was not observed.) ${ }^{19} \mathrm{~F}$

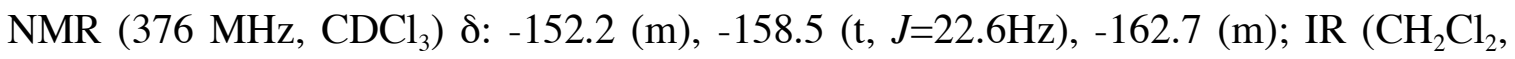
$\left.\mathrm{cm}^{-1}\right): 3505,2987,1787,1702,1522,1458,1421,1387,1328,1265,1035,999,896,748$ $[\alpha]_{\mathrm{D}}{ }^{25}=-24.6^{\circ}\left(\mathrm{c} 0.50, \mathrm{CH}_{2} \mathrm{Cl}_{2}\right)$; HRMS (ESI+) m/z: $[\mathrm{M}+\mathrm{Na}]$ calcd for $\mathrm{C}_{24} \mathrm{H}_{18} \mathrm{~F}_{5} \mathrm{NO}_{5}$ : 518.1003; found: 518.1025 .

\section{Pentafluorophenyl 2-methoxymethyl-1-(2-oxo-4S-phenyl-oxazolidine-3-carbonyl)} -cycloprop-2-ene carboxylic ester. [5f(major) \& 5f(minor)]

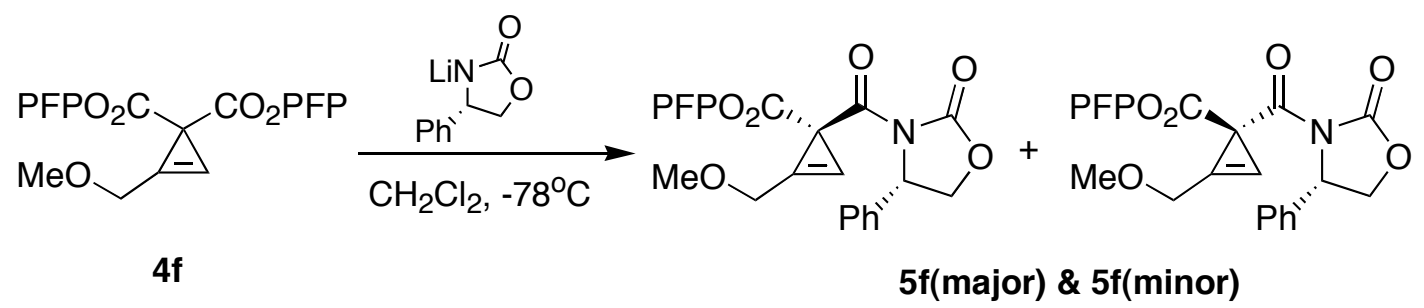


The general procedure for desymmetrization with $4 \mathbf{f}(86 \mathrm{mg}, 0.17 \mathrm{mmol})$ gave $65 \mathrm{mg}$ (79.5\%) of $\mathbf{5 f ( m a j o r ) ~ a n d ~} 10 \mathrm{mg}(12 \%)$ of $\mathbf{5 f ( m i n o r ) . ~ A ~ s i m i l a r ~ r e a c t i o n ~ s t a r t i n g ~ f r o m ~}$ $205 \mathrm{mg} \mathbf{4 f}$ gave $150 \mathrm{mg}(78 \%)$ of $\mathbf{5 f}$ (major) and $19 \mathrm{mg}(10 \%)$ of $\mathbf{5 f}($ minor). The diastereomeric ratio of the crude mixture was determined to be $85: 15$ by HPLC analysis (15\% isopropanol in hexane at $1 \mathrm{~mL} / \mathrm{min}$ ).

5f(major). semisolid; ${ }^{1} \mathrm{H}$ NMR (400 MHz, $\left.\mathrm{CDCl}_{3}\right) \delta: 7.35(\mathrm{~m}, 5 \mathrm{H}), 6.92$ (app t, $J=1.7 \mathrm{~Hz}$, $1 \mathrm{H}), 5.44(\mathrm{dd}, J=9.0 \mathrm{~Hz}, 5.3 \mathrm{~Hz}, 1 \mathrm{H}), 4.77$ (app t, $J=9.0 \mathrm{~Hz}, 1 \mathrm{H}), 4.66(\mathrm{dd}, J=16.5 \mathrm{~Hz}$, $1.6 \mathrm{~Hz}, 1 \mathrm{H}), 4.55(\mathrm{dd}, J=16.5 \mathrm{~Hz}, 1.8 \mathrm{~Hz}, 1 \mathrm{H}), 4.31(\mathrm{dd}, J=9.0 \mathrm{~Hz}, 5.2 \mathrm{~Hz}, 1 \mathrm{H}), 3.45(\mathrm{~s}, 3 \mathrm{H})$;

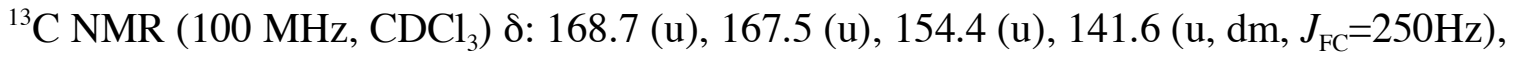
$140.0\left(\mathrm{u}, \mathrm{dm}, J_{\mathrm{FC}}=250 \mathrm{~Hz}\right), 138.3(\mathrm{u}), 138.2\left(\mathrm{u}, \mathrm{dm}, J_{\mathrm{FC}}=240 \mathrm{~Hz}\right), 129.6(\mathrm{dn}), 129.3(\mathrm{dn})$, $126.5(\mathrm{dn}), 125.2(\mathrm{u}, \mathrm{m}), 113.1(\mathrm{u}), 97.6(\mathrm{u}), 71.0(\mathrm{u}), 65.8(\mathrm{u}), 59.3(\mathrm{dn}), 58.2(\mathrm{dn}), 35.8$ (u); ${ }^{19} \mathrm{~F}$ NMR (376 MHz, $\left.\mathrm{CDCl}_{3}\right):-152.3(\mathrm{~m}),$,-158.1 (t, J=22.6Hz), -162.6 (m); IR $\left(\right.$ neat, $\left.\mathrm{cm}^{-1}\right): 1788,1705,1522,1390,1331,1194,1111,1030,1002 ;[\alpha]_{\mathrm{D}}^{25}=-25.2^{\circ}(\mathrm{c}$ 1.00, $\mathrm{CH}_{2} \mathrm{Cl}_{2}$ ); HRMS (ESI+) m/z: [M+Na] calcd for $\mathrm{C}_{22} \mathrm{H}_{14} \mathrm{~F}_{5} \mathrm{NO}_{6}$ : 506.0639; found: 506.0657 .

5f(minor). semisolid; ${ }^{1} \mathrm{H}$ NMR (400 $\left.\mathrm{MHz}, \mathrm{CDCl}_{3}\right) \delta: 7.36(\mathrm{~m}, 5 \mathrm{H}), 6.90$ (app t, $J=1.5 \mathrm{~Hz}$, $1 \mathrm{H}), 5.43(\mathrm{dd}, J=8.9,4.8 \mathrm{~Hz}, 1 \mathrm{H}), 4.77(\operatorname{app~t}, J=8.9 \mathrm{~Hz}, 1 \mathrm{H}), 4.65(\mathrm{dd}, J=16.2,1.5 \mathrm{~Hz}$, $1 \mathrm{H}), 4.54(\mathrm{dd}, J=16.2 \mathrm{~Hz}, 1.5 \mathrm{~Hz}, 1 \mathrm{H}), 4.31(\mathrm{dd}, J=8.9 \mathrm{~Hz}, 4.8 \mathrm{~Hz}), 3.41(\mathrm{~s}, 3 \mathrm{H}) ;{ }^{13} \mathrm{C} \mathrm{NMR}$ $\left(100 \mathrm{MHz}, \mathrm{CDCl}_{3}\right) \delta: 168.8(\mathrm{u}), 167.4(\mathrm{u}), 154.2(\mathrm{u}), 141.6\left(\mathrm{u}, \mathrm{dm}, J_{\mathrm{FC}}=260 \mathrm{~Hz}\right), 139.9(\mathrm{u}$, $\left.\mathrm{dm}, J_{\mathrm{FC}}=255 \mathrm{~Hz}\right), 138.5(\mathrm{u}), 137.9\left(\mathrm{u}, \mathrm{dm}, J_{\mathrm{FC}}=240 \mathrm{~Hz}\right), 129.7(\mathrm{dn}), 129.3(\mathrm{dn}), 126.3(\mathrm{dn})$, $125.3(\mathrm{u}, \mathrm{m}), 113.3(\mathrm{u}), 98.2(\mathrm{u}), 71.0(\mathrm{u}), 65.6(\mathrm{u}), 59.3(\mathrm{dn}), 58.1(\mathrm{dn}), 35.7(\mathrm{u}) ;{ }^{19} \mathrm{~F}$

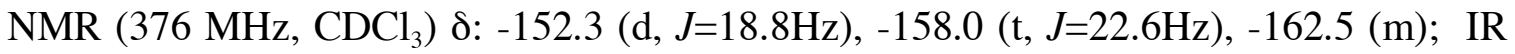
$\left(\right.$ neat, $\left.\mathrm{cm}^{-1}\right): 1789,1702,1523,1388,1331,1209,1113,1036,1001 ;[\alpha]_{\mathrm{D}}^{25}=-11.0^{\circ}(\mathrm{c}$ 
0.50, $\mathrm{CH}_{2} \mathrm{Cl}_{2}$ ); HRMS (ESI+) m/z: [M+Na] calcd for $\mathrm{C}_{22} \mathrm{H}_{14} \mathrm{~F}_{5} \mathrm{NO}_{6}$ : 506.0639; found: 506.0652.

\section{Pentafluorophenyl 1-(2-oxo-4S-phenyloxazolidine-3-carbonyl)-2-trityloxymethyl} -cycloprop-2-ene carboxylic ester [5g(major) \& 5g(minor)]

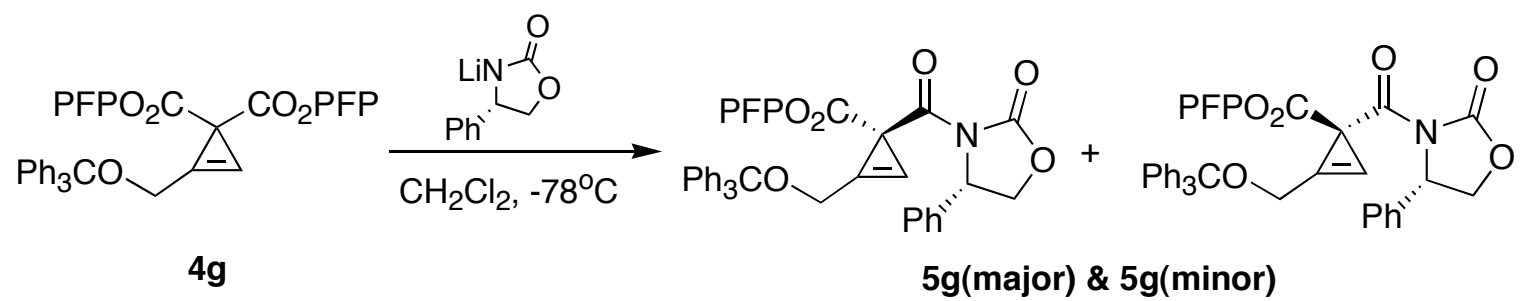

The general procedure for desymmetrization with $4 \mathrm{~g}$ (102 $\mathrm{mg}, 0.14 \mathrm{mmol})$ gave $61 \mathrm{mg}$ $(61 \%)$ of $\mathbf{5 g}$ (major) and $8 \mathrm{mg}(8 \%)$ of $\mathbf{5 g}$ (minor). A similar reaction starting from 106 $\mathrm{mg}$ of $\mathbf{4 g}$ gave $65 \mathrm{mg}(65 \%)$ of $\mathbf{5} \mathbf{g}$ (major) and $6 \mathrm{mg}(6 \%)$ of $\mathbf{5 g}$ (minor). The diastereomeric ratio of the crude mixture was determined to be $86: 14$ by HPLC analysis (5\% isopropanol in hexane at $1 \mathrm{~mL} / \mathrm{min}$ ).

5g(major). Semisolid; ${ }^{1} \mathrm{H}$ NMR (400 MHz, $\mathrm{CDCl}_{3}$ ) $\delta: 7.40$ (m, 20H), $6.72(\mathrm{~s}, 1 \mathrm{H}), 5.42$ (dd, $J=8.9 \mathrm{~Hz}, 5.1 \mathrm{~Hz}, 1 \mathrm{H}), 4.67($ app t $, J=8.9 \mathrm{~Hz}, 1 \mathrm{H}), 4.57(\mathrm{dd}, J=16.0 \mathrm{~Hz}, 1.3 \mathrm{~Hz}, 1 \mathrm{H})$, $4.33(\mathrm{dd}, J=16.0 \mathrm{~Hz}, 1.6 \mathrm{~Hz}, 1 \mathrm{H}), 4.28(\mathrm{dd}, J=8.9 \mathrm{~Hz}, 5.1 \mathrm{~Hz}, 1 \mathrm{H}) ;{ }^{13} \mathrm{C}$ NMR $(100 \mathrm{MHz}$, $\left.\mathrm{CDCl}_{3}\right) \delta: 168.5(\mathrm{u}), 167.5(\mathrm{u}), 154.2(\mathrm{u}), 143.7(\mathrm{u}), 141.1\left(\mathrm{u}, \mathrm{dm}, J_{\mathrm{FC}}=250 \mathrm{~Hz}\right), 139.5(\mathrm{u}$, $\left.\mathrm{dm}, J_{\mathrm{FC}}=250 \mathrm{~Hz}\right), 138.5(\mathrm{u}), 137.5\left(\mathrm{u}, \mathrm{dm}, J_{\mathrm{FC}}=256 \mathrm{~Hz}\right), 129.6(\mathrm{dn}), 129.3(\mathrm{dn}), 129.0(\mathrm{dn})$, $128.5(\mathrm{dn}), 127.8(\mathrm{dn}), 126.5(\mathrm{dn}), 125.6(\mathrm{u}, \mathrm{m}), 113.6(\mathrm{u}), 96.8(\mathrm{u}), 88.4(\mathrm{u}), 70.9(\mathrm{u})$, $58.6(\mathrm{u}), 58.1(\mathrm{dn}), 36.0(\mathrm{dn}) ;{ }^{19} \mathrm{~F}$ NMR $\left(376 \mathrm{MHz}, \mathrm{CDCl}_{3}\right) \delta:-152.0(\mathrm{~d}, J=18.8 \mathrm{~Hz})$, $-158.3(\mathrm{t}, J=22.6 \mathrm{~Hz}),-162.6(\mathrm{~m})$; IR (neat, $\left.\mathrm{cm}^{-1}\right): 1788,1705,1520,1450,1388,1329$, $1218,1156,1109,1080,1032,1000,764,738,706 ;[\alpha]_{\mathrm{D}}{ }^{25}=-6.5^{\circ}$ (c 1.00, THF); HRMS (ESI+) m/z: [M+Na] calcd for $\mathrm{C}_{40} \mathrm{H}_{26} \mathrm{~F}_{5} \mathrm{NO}_{6}: 734.1602$; found: 734.1609 . 
$\mathbf{5 g}$ (minor). (The purity of the compound was judged to be $90 \%$ by ${ }^{1} \mathrm{H}$ NMR. Minor impurities were detected in the ${ }^{1} \mathrm{H}$ NMR at $4.14 \mathrm{ppm}, 0.87-2.15 \mathrm{ppm}$. Further purification was not pursued for the minor diastereomer.) ${ }^{1} \mathrm{H} \mathrm{NMR}\left(400 \mathrm{MHz}, \mathrm{CDCl}_{3}\right.$ ) $\delta: 7.21-7.45$ $(\mathrm{m}, 20 \mathrm{H}), 6.89(\mathrm{t}, J=1.6 \mathrm{~Hz}, 1 \mathrm{H}), 5.41(\mathrm{dd}, J=8.9 \mathrm{~Hz}, 5.0 \mathrm{~Hz}, 1 \mathrm{H}), 4.74$ (appt, $J=8.9 \mathrm{~Hz}$, $1 \mathrm{H}), 4.39(\mathrm{~d}, J=1.6 \mathrm{~Hz}, 2 \mathrm{H}), 4.27(\mathrm{dd}, J=8.9 \mathrm{~Hz}, 5.0 \mathrm{~Hz}, 1 \mathrm{H}) ;) ;{ }^{13} \mathrm{C} \mathrm{NMR}(100 \mathrm{MHz}$,

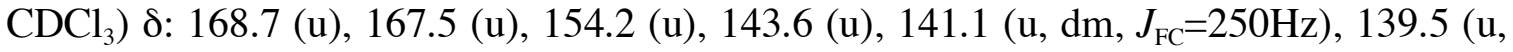
$\left.\mathrm{dm}, J_{\mathrm{FC}}=250 \mathrm{~Hz}\right), 138.5(\mathrm{u}), 137.5\left(\mathrm{u}, \mathrm{dm}, J_{\mathrm{FC}}=250 \mathrm{~Hz}\right), 129.6(\mathrm{dn}), 129.2(\mathrm{dn}), 129.0(\mathrm{dn})$, $128.4(\mathrm{dn}), 127.7(\mathrm{dn}), 126.4(\mathrm{dn}), 125.6(\mathrm{u}, \mathrm{m}), 113.9(\mathrm{u}), 97.1(\mathrm{u}), 88.3(\mathrm{u}), 70.9(\mathrm{u})$, $58.9(\mathrm{u}), 58.1(\mathrm{dn}), 36.1(\mathrm{dn}) ; \quad{ }^{19} \mathrm{~F}$ NMR $\left(376 \mathrm{MHz}, \mathrm{CDCl}_{3}\right) \quad \delta:-151.9(\mathrm{~d}, J=18.8 \mathrm{~Hz})$, $-158.3(\mathrm{t}, J=22.6 \mathrm{~Hz}),-162.7(\mathrm{~m})$; IR $\left(\mathrm{CHCl}_{3}, \mathrm{~cm}^{-1}\right): 1785,1762,1702,1522,1387,1329$, 1216, 1080, 1035, 1000, 907, 733, 650; HRMS (ESI+) m/z: [M+Na] calcd for $\mathrm{C}_{40} \mathrm{H}_{26} \mathrm{~F}_{5} \mathrm{NO}_{6}:$ : 734.1602; found: 734.1607 .

\section{2-Butyl-1-(2-oxo-4S-phenyloxazolidine-3-carbonyl)-cycloprop-2-ene carbonyl azide} (6a)

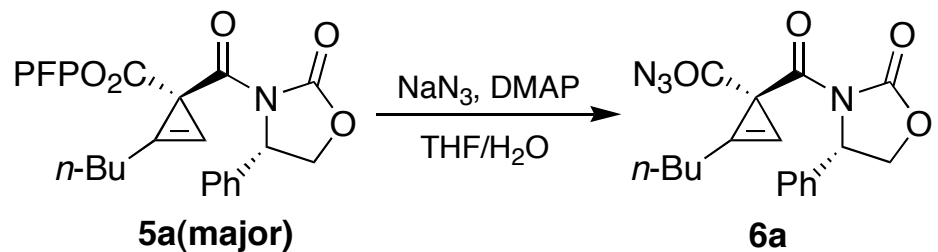

To a solution of $\mathbf{5 a}$ (major) $(3.72 \mathrm{~g}, 7.51 \mathrm{mmol})$ in $\mathrm{THF} / \mathrm{H}_{2} \mathrm{O}(5: 1,120 \mathrm{~mL})$ was added $\mathrm{NaN}_{3}(2.44 \mathrm{~g}, 37.6 \mathrm{mmol})$ and DMAP (49 mg, $\left.0.4 \mathrm{mmol}\right)$. The reaction mixture was vigorously stirred for $15 \mathrm{~h}$ at $\mathrm{rt}$. The mixture was then concentrated to remove $\mathrm{THF}$, diluted with water $(50 \mathrm{~mL})$ and extracted with ether $(200 \mathrm{~mL})$. The ether layer was then washed with water $(50 \mathrm{~mL})$ and brine $(50 \mathrm{~mL})$, dried over $\mathrm{Na}_{2} \mathrm{SO}_{4}$, filtered, concentrated 
and chromatographed (30\% ether in hexanes) to give $2.59 \mathrm{~g}(97 \%)$ of the title compound as semisolid. A similar reaction starting from $3.22 \mathrm{~g}$ of $\mathbf{5 3 a}$ (major) gave $2.18 \mathrm{~g}$ (95\%) of the title compound. ${ }^{1} \mathrm{H}$ NMR (400 MHz, $\left.\mathrm{CDCl}_{3}\right) \delta: 7.40(\mathrm{~m}, 5 \mathrm{H}), 6.43(\mathrm{t}, J=1.5 \mathrm{~Hz}, 1 \mathrm{H})$, $5.43(\mathrm{dd}, J=9.0 \mathrm{~Hz}, 5.4 \mathrm{~Hz}, 1 \mathrm{H}), 4.75(\operatorname{app~t}, J=9.0 \mathrm{~Hz}, 1 \mathrm{H}), 4.28(\mathrm{dd}, J=8.9 \mathrm{~Hz}, 5.4 \mathrm{~Hz}, 1 \mathrm{H})$, $2.56(\mathrm{~m}, 2 \mathrm{H}), 1.61(\mathrm{~m}, 2 \mathrm{H}), 1.38(\mathrm{~m}, 2 \mathrm{H}), 0.91(\mathrm{t}, J=7.3 \mathrm{~Hz}, 3 \mathrm{H}) ;{ }^{13} \mathrm{C} \mathrm{NMR}(100 \mathrm{MHz}$

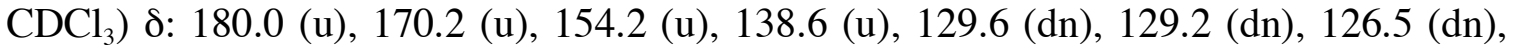
$117.2(u), 95.0(u), 70.9(u), 58.3(d n), 38.5(u), 29.0(u), 24.6(u), 22.6(u), 14.1(d n) ;$ IR $\left(\right.$ neat, $\left.\mathrm{cm}^{-1}\right): 2136,1779,1693,1458,1386,1322,1198,1101,958,763,701$; $[\alpha]_{\mathrm{D}}^{25}=-61.6^{\circ}$ (c 1.00, $\mathrm{CH}_{2} \mathrm{Cl}_{2}$ ); HRMS (CI) m/z: [M-CON 3 calcd for $\mathrm{C}_{18} \mathrm{H}_{18} \mathrm{~N}_{4} \mathrm{O}_{4}$ : 284.1287; found: 284.1299 .

\section{$1 R$-(2-Oxo-4S-phenyloxazolidine-3-carbonyl)-2-phenyl-cycloprop-2-ene carbonyl azide (6b)}

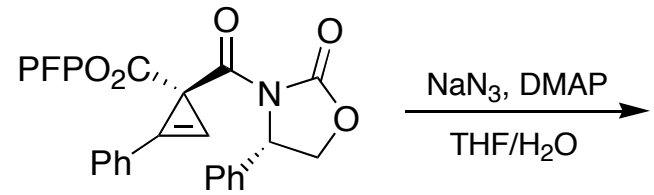

$5 b(R c y$, Sox $)$

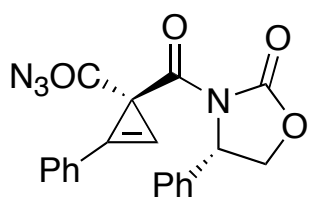

$6 b$

The procedure was identical to that used to prepare $\mathbf{6 a}$, except that $\mathbf{5 b}(\boldsymbol{R} \mathbf{c y}, \boldsymbol{S o x})(100$ $\mathrm{mg}, 0.2 \mathrm{mmol})$ was used. Other reagents were used in the following amounts: $\mathrm{NaN}_{3}(65$ $\mathrm{mg}, 1.0 \mathrm{mmol}), \mathrm{THF} / \mathrm{H}_{2} \mathrm{O}(5: 1,2.4 \mathrm{~mL})$, and DMAP $(2.7 \mathrm{mg}, 0.02 \mathrm{mmol})$. The protocol gave $72 \mathrm{mg}(96 \%)$ of the title compound as semisolid. A similar reaction starting from $235 \mathrm{mg}$ of $\mathbf{5 b}(\boldsymbol{R c y}, \boldsymbol{S o x})$ gave $163 \mathrm{mg}(95 \%)$ of the title compound. ${ }^{1} \mathrm{H}$ NMR (400 MHz, $\left.\mathrm{CDCl}_{3}\right) \delta: 7.75(\mathrm{~m}, 2 \mathrm{H}), 7.42(\mathrm{~m}, 8 \mathrm{H}), 6.92(\mathrm{~s}, 1 \mathrm{H}), 5.43(\mathrm{dd}, J=8.9 \mathrm{~Hz}, 4.7 \mathrm{~Hz}, 1 \mathrm{H}), 4.76$ (app t, $J=8.9 \mathrm{~Hz}, 1 \mathrm{H}), 4.29(\mathrm{dd}, J=8.9 \mathrm{~Hz}, 4.7 \mathrm{~Hz}, 1 \mathrm{H}) ;{ }^{13} \mathrm{C} \mathrm{NMR}\left(100 \mathrm{MHz}, \mathrm{CDCl}_{3}\right) \delta$ : 
$178.9(\mathrm{u}), 168.7(u), 153.9(u), 138.3(\mathrm{u}), 130.8(\mathrm{dn}), 130.6(\mathrm{dn}), 129.1(\mathrm{dn}), 128.8(\mathrm{dn})$, $128.7(\mathrm{dn}), 125.9(\mathrm{dn}), 123.8(\mathrm{u}), 113.4(\mathrm{u}), 96.1(\mathrm{u}), 70.5(\mathrm{u}), 57.9(\mathrm{dn}), 38.6(\mathrm{u})$; IR $\left(\right.$ neat, $\left.\mathrm{cm}^{-1}\right): 2142,1779,1694,1389,1329,1302,1230,1201,1107,911,766,732,699$; $[\alpha]_{\mathrm{D}}^{25}=+20.7^{\circ}$ (c 1.00, THF); HRMS (CI) m/z: [M-CON ${ }_{3}$ calcd for $\mathrm{C}_{20} \mathrm{H}_{14} \mathrm{~N}_{4} \mathrm{O}_{4}$ : 304.0960; found: 304.0965 .

(+)-4-Methoxybenzyl 2-butyl-1-(4-methoxybenzyloxycarbonylamino)-cycloprop-2ene carboxylic ester (7a)<smiles>CCCC1C[C@]1(C(N)=O)C(=O)N1C(=O)OC[C@H]1c1ccccc1</smiles>

$6 a$

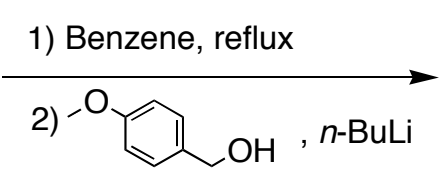

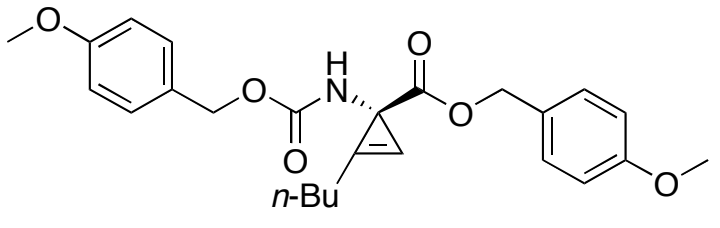

$7 a$

A solution of $6 \mathbf{a}(150 \mathrm{mg}, 0.46 \mathrm{mmol})$ in benzene $(2 \mathrm{~mL})$ was heated to reflux for $6 \mathrm{~h}$.

The reaction mixture was allowed to cool to rt, and was concentrated to remove benzene.

To the crude isocyanate was added THF $(5 \mathrm{~mL})$ and $p$-methoxylbenzyl alcohol $(190 \mathrm{mg}$, $1.38 \mathrm{mmol})$. The mixture was chilled in a bath of dry ice/acetone $\left(-78{ }^{\circ} \mathrm{C}\right)$, and $n$-BuLi ( $0.46 \mathrm{mmol}, 0.23 \mathrm{~mL}$ of a $2.0 \mathrm{M}$ pentane solution) was added dropwise via syringe to the mixture. The reaction mixture was allowed to stir at $-78{ }^{\circ} \mathrm{C}$ for $30 \mathrm{~min}$ and then allowed to warm to rt. The mixture was concentrated and chromatographed (10\% ethyl acetate in hexanes) to give $103 \mathrm{mg}(51 \%)$ of the title compound as semisolid. A similar reaction starting from $3.60 \mathrm{~g}$ of $\mathbf{6 a}$ gave $2.60 \mathrm{~g}$ (58\%) of the title compound. (4S)-4Phenyloxazolidinone was recovered in $75 \%$ yield. ${ }^{1} \mathrm{H}$ NMR $\left(400 \mathrm{MHz}, \mathrm{CDCl}_{3}\right)$ 8: 7.23 (m, 4H), $6.84(\mathrm{~m}, 4 \mathrm{H}), 6.30-6.62(\mathrm{~m}, 1 \mathrm{H}), 5.30-5.65(\mathrm{~m}, 1 \mathrm{H}), 5.03(\mathrm{~m}, 4 \mathrm{H}), 3.78(\mathrm{~s}, 6 \mathrm{H})$, 2.10-2.54 (m, 2H), $1.53(\mathrm{~m}, 2 \mathrm{H}), 1.35(\mathrm{~m}, 2 \mathrm{H}), 0.87(\mathrm{~m}, 3 \mathrm{H}) ;{ }^{13} \mathrm{C}$ NMR (100 MHz,

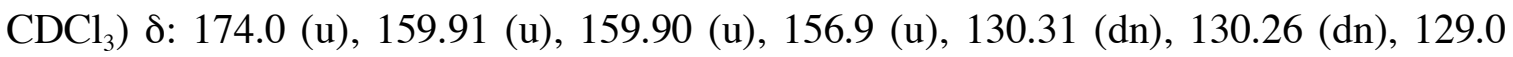


(u), $128.6(u), 121.1(u), 114.26(d n), 114.24(d n), 99.4(u), 67.2(u), 66.7(u), 55.7(d n)$, $38.8(u), 29.1(u), 24.4(u), 22.7(u), 14.1(d n) ; I R\left(\right.$ neat, $\left.\mathrm{cm}^{-1}\right): 3366,2955,1776,1713$, $1613,1515,1467,1303,1244,1175,1092,1032,823,737,669,604 ;[\alpha]_{\mathrm{D}}{ }^{25}=+20.0^{\circ}(\mathrm{c}$ 1.00, THF); HRMS (ESI+) m/z: [M+Na] calcd for $\mathrm{C}_{25} \mathrm{H}_{29} \mathrm{NO}_{6}$ : 462.1893; found: 462.1899 .

(+)-4-Methoxy-benzyl 1R-(4-methoxybenzyloxycarbonylamino)-2-phenyl-cycloprop2-ene carboxylic ester (7b)<smiles>N#C[C@H]1C(c2ccccc2)[C@@]1(C(=O)O)c1ccccc1</smiles>

6b

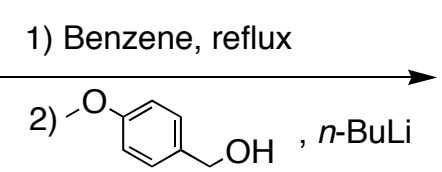

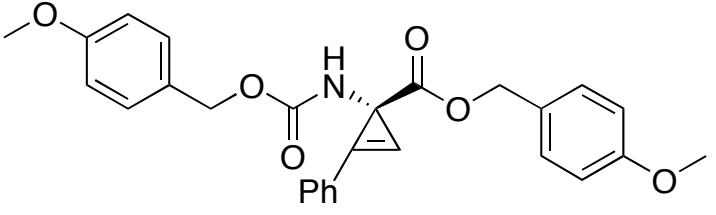

$7 b$

The procedure was identical to that used to prepare 7a, except that $\mathbf{6 b}(148 \mathrm{mg}, 0.39$ mmol) was used. Other reagents were used in the following amounts: benzene $(10 \mathrm{~mL})$, THF (5 mL), p-methoxylbenzyl alcohol (241 mg, $1.75 \mathrm{mmol})$, and $n$-BuLi (0.39 mmol, $0.2 \mathrm{~mL}$ of a $2.0 \mathrm{M}$ pentane solution). The protocol gave $75 \mathrm{mg}(42 \%)$ of the title compound as white solid. A similar reaction starting from $230 \mathrm{mg}$ of $\mathbf{6 b}$ gave $113 \mathrm{mg}$ (40\%) of the title compound. mp: $115-116^{\circ} \mathrm{C} ;{ }^{1} \mathrm{H}$ NMR (400 $\left.\mathrm{MHz}, \mathrm{CDCl}_{3}\right)$ o: 7.63 (m, 2H), $7.41(\mathrm{~m}, 3 \mathrm{H}), 7.25(\mathrm{~m}, 2 \mathrm{H}), 7.18(\mathrm{~m}, 2 \mathrm{H}), 7.10(\mathrm{~m}, 1 \mathrm{H}), 6.82(\mathrm{~m}, 4 \mathrm{H}), 5.69(\mathrm{bs}, 1 \mathrm{H})$, $5.08(\mathrm{~s}, 2 \mathrm{H}), 5.04(\mathrm{~d}, J=11.9 \mathrm{~Hz}, 1 \mathrm{H}), 5.00(\mathrm{~d}, J=11.9 \mathrm{~Hz}, 1 \mathrm{H}), 3.80(\mathrm{~s}, 3 \mathrm{H}), 3.79(\mathrm{~s}, 3 \mathrm{H})$

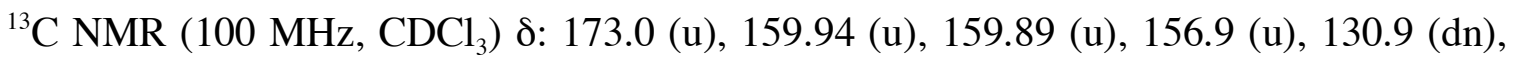
$130.7(\mathrm{dn}), 130.4(\mathrm{dn}), 130.1(\mathrm{dn}), 129.2(\mathrm{dn}), 128.9(\mathrm{u}), 128.5(\mathrm{u}), 124.7(\mathrm{u}), 119.4(\mathrm{u})$, $114.3(\mathrm{dn}), 114.2(\mathrm{dn}), 100.4(\mathrm{u}), 67.4(\mathrm{u}), 66.9(\mathrm{u}), 55.69(\mathrm{dn}), 55.68(\mathrm{dn}), 38.9(\mathrm{u}) ;$ IR $\left(\right.$ neat, $\left.\mathrm{cm}^{-1}\right): 1715,1614,1516,1466,1303,1248,1177,1106,1034,825,728,711$; 
$[\alpha]_{\mathrm{D}}^{25}=+66.2^{\circ}\left(\mathrm{c} 1.00, \mathrm{CH}_{2} \mathrm{Cl}_{2}\right)$; HRMS (ESI+) m/z: [M+Na] calcd for $\mathrm{C}_{27} \mathrm{H}_{25} \mathrm{NO}_{6}$ : 482.1580; found: 482.1591 .

\section{2-Butyl-1-(4-methoxybenzyloxycarbonylamino)-cycloprop-2-ene carboxylic acid (8a)}

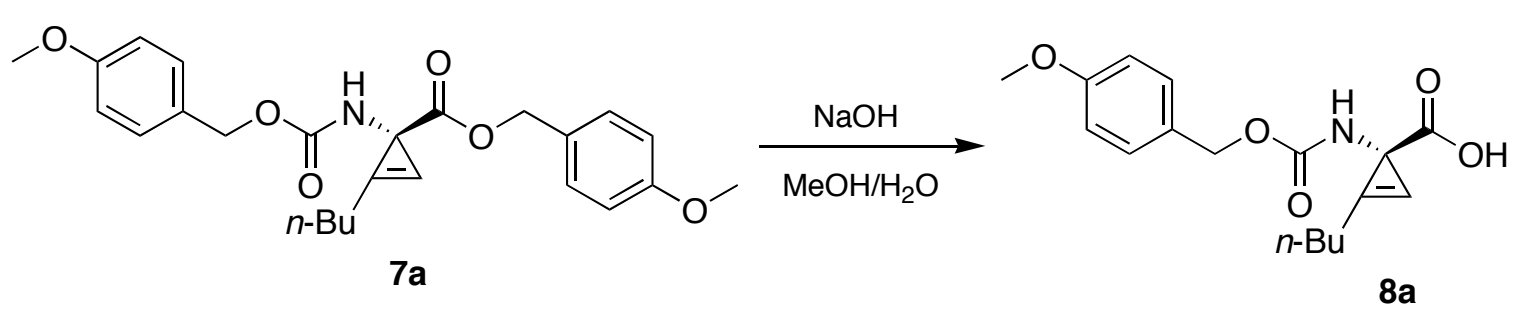

To a solution of 7a $(175 \mathrm{mg}, 0.40 \mathrm{mmol})$ in $\mathrm{MeOH}(5 \mathrm{~mL})$ was added dropwise $1 \mathrm{M}$ $\mathrm{NaOH}(2 \mathrm{~mL})$ at $0^{\circ} \mathrm{C}$. The reaction mixture was allowed to stir at $\mathrm{rt}$ for $14 \mathrm{~h}$. The mixture was then concentrated to remove $\mathrm{MeOH}$ and diluted with water $(5 \mathrm{~mL})$. The mixture was extracted with ethyl acetate $(3 \times 5 \mathrm{~mL})$. The aqueous layer was acidified with $10 \% \mathrm{HCl}$ (aq) and then extracted with ethyl acetate $(3 \times 10 \mathrm{~mL})$. The organics were combined, dried over $\mathrm{Na}_{2} \mathrm{SO}_{4}$, filtered and concentrated to give $120 \mathrm{mg}(96 \%)$ of the title compound as semisolid. A similar reaction starting from $239 \mathrm{mg}$ of $7 \mathbf{a}$ gave $155 \mathrm{mg}(90 \%)$ of the title compound. ${ }^{1} \mathrm{H}$ NMR (400 MHz, dmso- $\left.d 6,318 \mathrm{~K}\right) \delta: 7.56$ (bs, 1H), 7.24 (d, J=8.7Hz, 2H), 6.95 (s, 1H), 6.87 (d, J=8.7Hz, 2H), 4.89 (s, 2H), $3.72(\mathrm{~s}, 3 \mathrm{H}), 2.46(\mathrm{~m}, 2 \mathrm{H}), 1.49$

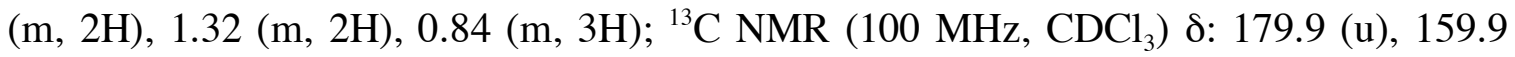
(u), 157.0 (u), $130.4(\mathrm{dn}), 128.9$ (u), 120.7 (u), $114.3(\mathrm{dn}), 99.1$ (u), 66.9 (u), 55.7 (dn), $38.2(u), 29.1(u), 24.4(u), 22.7(u), 14.1(\mathrm{dn})$; IR (neat, $\left.\mathrm{cm}^{-1}\right)$ : 2959, 1712, 1695, 1614, $1517,1465,1327,1303,1248,1177,1035,822,778,755,738 ;[\alpha]_{\mathrm{D}}{ }^{25}=+19.6^{\circ}(\mathrm{c} 1.00$, THF); HRMS (ESI+) m/z: [M+Na] calcd for $\mathrm{C}_{17} \mathrm{H}_{21} \mathrm{NO}_{5}: 342.1317$; found: 342.1315 . 


\section{1-Amino-2-butyl-cycloprop-2-ene carboxylic acid (10a)}

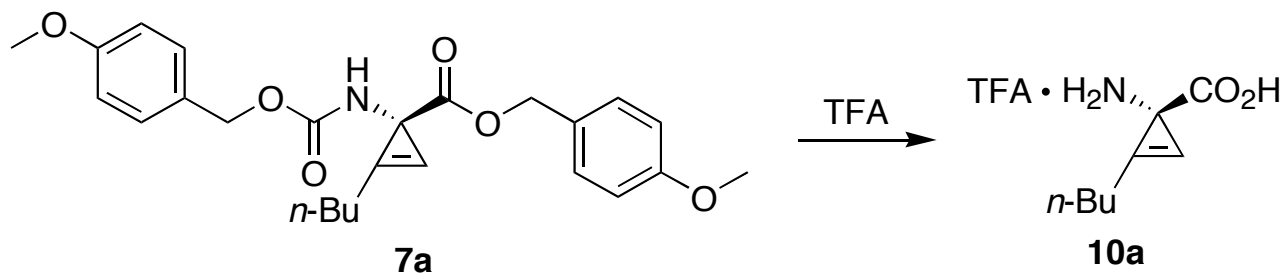

A solution of $7 \mathbf{a}(2.38 \mathrm{~g}, 5.44 \mathrm{mmol})$ in $100 \mathrm{~mL}$ of a TFA $\left(20 \%\right.$ in $\left.\mathrm{CH}_{2} \mathrm{Cl}_{2}\right)$ solution was allowed to stir at $\mathrm{rt}$ for $1 \mathrm{~h}$. The mixture was concentrated, and water $(150 \mathrm{~mL})$ was added. The aqueous solution was extracted with ether $(3 \times 20 \mathrm{~mL})$. The aqueous layer was concentrated on a lyopholyzer to give $1.22 \mathrm{~g}(84 \%)$ of the TFA salt of the title compound as white solid. A similar reaction starting from $3.39 \mathrm{~g}$ of $\mathbf{7 a}$ gave $1.78 \mathrm{~g}(86 \%)$ of the title compound. mp: 95-96 ${ }^{\circ} \mathrm{C} ;{ }^{1} \mathrm{H}$ NMR $\left(400 \mathrm{MHz}, \mathrm{D}_{2} \mathrm{O}\right) \delta: 6.80(\mathrm{t}, J=1.3 \mathrm{~Hz}, 1 \mathrm{H}), 2.44(\mathrm{~m}$, 2H), $1.44(\mathrm{~m}, 2 \mathrm{H}), 1.22(\mathrm{~m}, 2 \mathrm{H}), 0.72(\mathrm{t}, J=7.3 \mathrm{~Hz}, 3 \mathrm{H}) ;{ }^{13} \mathrm{C} \mathrm{NMR}\left(90 \mathrm{MHz}, \mathrm{D}_{2} \mathrm{O}\right) \delta$ : $176.8(u), 122.4(u), 101.1(u), 41.7(u), 30.4(u), 25.3(u), 24.3(u), 15.6(d n) ;$ IR (neat, $\left.\mathrm{cm}^{-1}\right): 1731,1660,1525,1271,1180,1141,1085,986,834,798,722 ;[\alpha]_{\mathrm{D}}^{25}=-16.0^{\circ}(\mathrm{c}$ 1.00, acetone); HRMS (CI) m/z: [M+H] calcd for $\mathrm{C}_{8} \mathrm{H}_{13} \mathrm{NO}_{2}$ : 156.1024; found: 156.1018.

\section{$1 R$-Amino-2-phenyl-cycloprop-2-ene carboxylic acid (10b)}

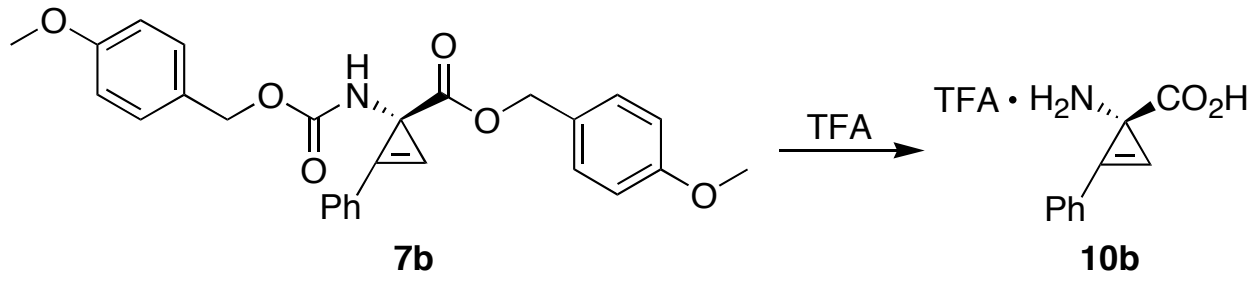

The procedure was identical to that used to prepare 10a, except that $7 \mathbf{b}(76 \mathrm{mg}, 0.16$ $\mathrm{mmol}$ ), and $2 \mathrm{~mL}$ of the TFA solution (20\% in $\mathrm{CH}_{2} \mathrm{Cl}_{2}$ ). The protocol gave $35 \mathrm{mg}(76 \%)$ 
of the TFA salt of the title compound as white solid. A identical reaction also gave $35 \mathrm{mg}$ (76\%) of the title compound. mp: $108-109^{\circ} \mathrm{C} ;{ }^{1} \mathrm{H}$ NMR $\left(400 \mathrm{MHz}, \mathrm{D}_{2} \mathrm{O}\right) \delta: 7.55(\mathrm{~m}, 2 \mathrm{H})$, $7.42(\mathrm{~m}, 3 \mathrm{H}), 7.29(\mathrm{~s}, 1 \mathrm{H}) ;{ }^{13} \mathrm{C}$ NMR (100 MHz, D $\left.\mathrm{O}\right) \delta: 174.0(\mathrm{u}), 132.2(\mathrm{dn}), 130.5$ (dn), $129.7(\mathrm{dn}), 122.6(\mathrm{u}), 116.5(\mathrm{u}), 99.4(\mathrm{u}), 39.0(\mathrm{u})$; IR (neat, $\mathrm{cm}^{-1}$ ): 1660, 1503, $1277,1182,1134,1091,992,935,722,688 ;[\alpha]_{\mathrm{D}}^{25}=-6.9^{\circ}$ (c 1.00, acetone); HRMS (CI) m/z: $[\mathrm{M}+\mathrm{H}]$ calcd for $\mathrm{C}_{10} \mathrm{H}_{9} \mathrm{NO}_{2}$ : 176.0711; found: 176.0706 .

\section{2-Butyl-1-(9H-fluoren-9-ylmethoxycarbonylamino)-cycloprop-2-ene carboxylic acid} (9a)
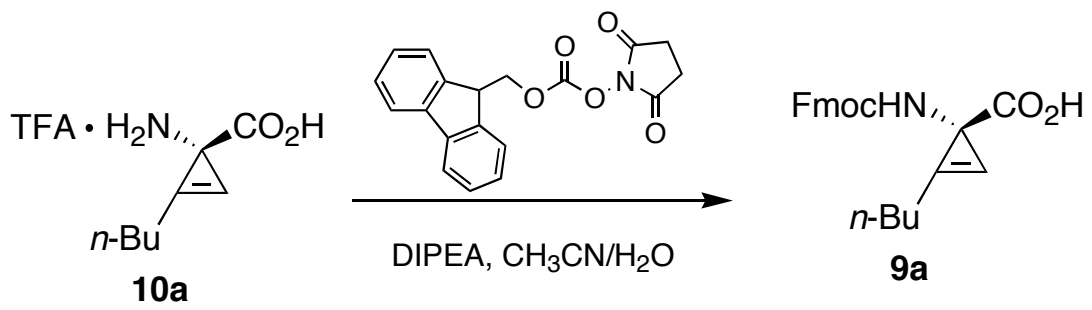

A solution of $10 \mathbf{a}(70 \mathrm{mg}, 0.26 \mathrm{mmol})$ in $\mathrm{CH}_{3} \mathrm{CN}(2 \mathrm{~mL})$ and $\mathrm{H}_{2} \mathrm{O}(2 \mathrm{~mL})$ was added Fmoc-OSu (88 mg, $0.26 \mathrm{mmol})$ and diisopropylethylamine (101 mg, $0.78 \mathrm{mmol})$. The reaction mixture was stirred at room temperature for 4 hours, after which time the solvent was removed under reduced pressure. The residue was partitioned between ethyl acetate $(5 \mathrm{~mL})$ and $0.1 \mathrm{M}$ citric acid $(5 \mathrm{~mL})$. The organic layer was washed with $0.1 \mathrm{M}$ citric acid $(2 \times 5 \mathrm{~mL})$, water $(5 \mathrm{~mL})$, dried over $\mathrm{Na}_{2} \mathrm{SO}_{4}$, filtered and concentrated under vacuum to give $90 \mathrm{mg}(92 \%)$ of the title compound as white solid. A similar reaction starting from $1.72 \mathrm{~g}$ of $10 \mathrm{a}$ gave $2.4 \mathrm{~g}(100 \%)$ of the title compound. mp:48-50 ${ }^{\circ} \mathrm{C} ;{ }^{1} \mathrm{H}$ NMR (400 MHz, dmso- d6, 373K) $\delta: 7.81(\mathrm{~m}, 2 \mathrm{H}), 7.64(\mathrm{~m}, 2 \mathrm{H}), 7.33(\mathrm{~m}, 5 \mathrm{H}), 6.81(\mathrm{~s}, 1 \mathrm{H}), 4.24$ $(\mathrm{m}, 3 \mathrm{H}), 2.45(\mathrm{~m}, 2 \mathrm{H}), 1.52(\mathrm{~m}, 2 \mathrm{H}), 1.34(\mathrm{~m}, 2 \mathrm{H}), 0.85(\mathrm{~m}, 3 \mathrm{H}) ;{ }^{13} \mathrm{C} \mathrm{NMR}(100 \mathrm{MHz}$, 


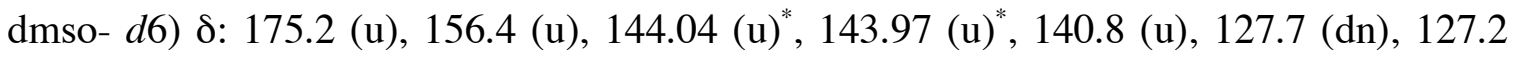
(dn), $125.4(\mathrm{dn}), 120.4(\mathrm{u}), 120.2(\mathrm{dn}), 100.6(\mathrm{u}), 65.4(\mathrm{u}), 46.8(\mathrm{dn}), 36.7(\mathrm{u}), 28.4(\mathrm{u})$, $23.6(\mathrm{u}), 21.7(\mathrm{u}), 13.7(\mathrm{dn})$; IR $\left(\mathrm{CHCl}_{3}, \mathrm{~cm}^{-1}\right):$ 3438, 3155, 2961, 2253, 1720, 1701, $1491,1450,1229,1094,912,742,650 ;[\alpha]_{\mathrm{D}}{ }^{25}=+6.13^{\circ}\left(\mathrm{c} 1.50, \mathrm{CHCl}_{3}\right) ; \mathrm{HRMS}(\mathrm{ESI}+)$ m/z: [M+Na] calcd for $\mathrm{C}_{23} \mathrm{H}_{23} \mathrm{NO}_{4}$ : 400.1525; found: 400.1523 .

"Note: The peaks in the ${ }^{13} \mathrm{C}$ NMR spectrum at 144.04 and $143.97 \mathrm{ppm}$ are assigned to carbons of the Fmoc group with a diastereotopic relationship.

\section{Methyl \{[2-butyl-1-(4-methoxybenzyloxycarbonylamino)-cycloprop-2-ene}

\section{carbonyl]-amino\}-acetic ester (11)}

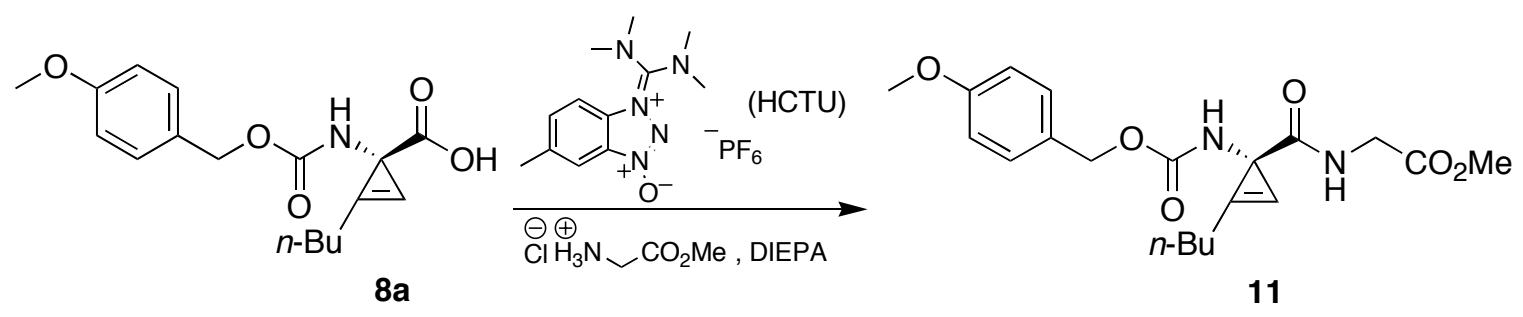

Diisopropylethylamine $(66 \mathrm{mg}, 0.51 \mathrm{mmol})$ was added to a solution of $\mathbf{8 a}(55 \mathrm{mg}, 0.17$ mmol), Glycine methyl ester hydrochloride (22 mg, $0.17 \mathrm{mmol}$ ), and HCTU (84 mg, 0.2 mmol) in $\mathrm{CH}_{3} \mathrm{CN}(1 \mathrm{~mL})$. The reaction mixture was allowed to stir at $\mathrm{rt}$ for $3 \mathrm{~h}$. The mixture was then concentrated and chromatographed (eluting first with $20 \%$ ethyl acetate in hexanes, and then $20 \%$ methanol in $\mathrm{CH}_{2} \mathrm{Cl}_{2}$ ) to give $59 \mathrm{mg}$ of title compound, which was contaminated by $\sim 20 \%$ tetramethylurea. A similar reaction starting from $50 \mathrm{mg}$ of $\mathbf{8 a}$ gave $58 \mathrm{mg}$ of $\mathbf{1 1}$. Further chromatography did not remove the tetramethylurea, so $\mathbf{1 1}$ was used in next reaction without further purification. ${ }^{1} \mathrm{H}$ NMR $\left(400 \mathrm{MHz}, \mathrm{CDCl}_{3}\right) \delta: 7.30$ $(\mathrm{m}, 2 \mathrm{H}), 6.88(\mathrm{~m}, 2 \mathrm{H}), 6.75(\mathrm{bs}, 1 \mathrm{H}), 6.60(\mathrm{bs}, 1 \mathrm{H}), 5.80(\mathrm{~s}, 1 \mathrm{H}), 5.03(\mathrm{~m}, 2 \mathrm{H}), 4.03(\mathrm{~m}$, 2H), $3.82(\mathrm{~s}, 3 \mathrm{H}), 3.76(\mathrm{~s}, 3 \mathrm{H}), 2.59(\mathrm{~m}, 2 \mathrm{H}), 1.61(\mathrm{~m}, 2 \mathrm{H}), 1.40(\mathrm{~m}, 2 \mathrm{H}), 0.93(\mathrm{~m}, 3 \mathrm{H})$; 


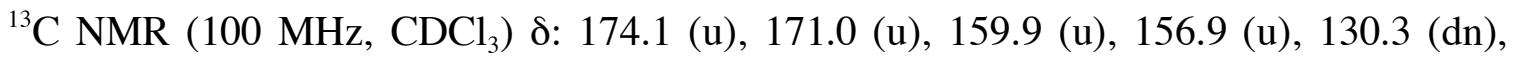
$128.8(u), 122.0(\mathrm{u}), 114.2(\mathrm{dn}), 100.0(\mathrm{u}), 66.8(\mathrm{u}), 55.6(\mathrm{dn}), 52.6(\mathrm{dn}), 42.0(\mathrm{u}), 39.9$ (u), $29.2(u), 24.3(u), 22.7(u), 14.1(\mathrm{dn}) ;$ HRMS (ESI+) m/z: [M+Na] calcd for $\mathrm{C}_{20} \mathrm{H}_{26} \mathrm{~N}_{2} \mathrm{O}_{6}$ : 413.1689; found: 413.1693 .

\section{Methyl [(1-amino-2-butyl-cycloprop-2-enecarbonyl)-amino]-acetic ester (12)}

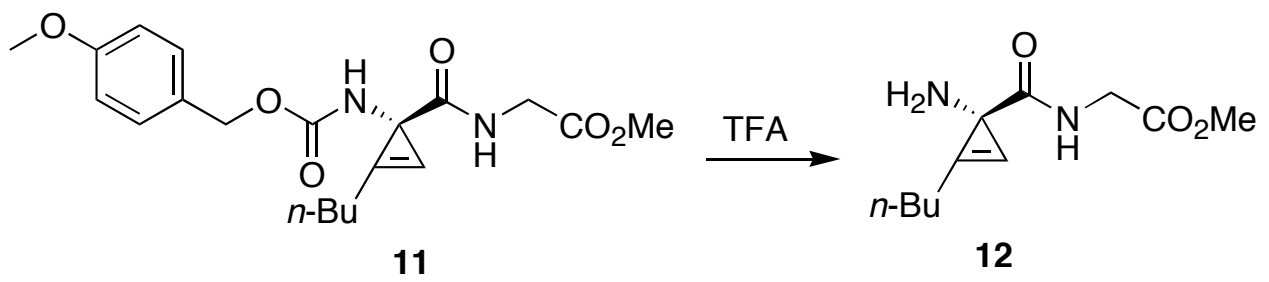

A solution of 11 (59 mg, contaminated by $20 \%$ tetramethylurea as described above) in $5 \%$ anisole in TFA $(2 \mathrm{~mL})$ was chilled in an ice/water bath and allowed to stir for $1 \mathrm{~h}$. The reaction mixture was then concentrated and chromatographed [TFA/MeOH/ $\mathrm{CH}_{2} \mathrm{Cl}_{2}$ (0.6:10:90) was the eluent.] to give $34 \mathrm{mg}(88 \%$ from 11) of the title compound as semisolid. ${ }^{1} \mathrm{H}$ NMR (400 MHz, $\left.\mathrm{CD}_{3} \mathrm{CN}\right)$ 8: 8.0 (bs, 2H), $6.99(\mathrm{~s}, 1 \mathrm{H}), 6.72(\mathrm{~s}, 1 \mathrm{H}), 3.98$ (dd, $J=17.6 \mathrm{~Hz}, 6.2 \mathrm{~Hz}, 1 \mathrm{H}), 3.89$ (dd, J=17.6Hz, 5.8Hz, 1H), $3.68(\mathrm{~s}, 3 \mathrm{H}), 2.60(\mathrm{~m}, 2 \mathrm{H})$, $1.66(\mathrm{~m}, 2 \mathrm{H}), 1.45(\mathrm{~m}, 2 \mathrm{H}), 0.95(\mathrm{t}, J=7.3 \mathrm{~Hz}, 3 \mathrm{H}) ;{ }^{13} \mathrm{C} \mathrm{NMR}\left(100 \mathrm{MHz}, \mathrm{CD}_{3} \mathrm{CN}\right) \delta$ : $172.1(\mathrm{u}), 170.4(\mathrm{u}), 121.5(\mathrm{u}), 99.8(\mathrm{u}), 52.2(\mathrm{dn}), 41.3(\mathrm{u}), 40.2(\mathrm{u}), 28.4(\mathrm{u}), 23.6(\mathrm{u})$, $22.3(\mathrm{u}), 13.4(\mathrm{dn}) ; \mathrm{IR}\left(\mathrm{CH}_{3} \mathrm{CN}, \mathrm{cm}^{-1}\right): 3413,2964,1745,1680,1538,1440,1376,1204$, $1139,839,801,723 ;[\alpha]_{\mathrm{D}}{ }^{25}=-9.9^{\circ}\left(\mathrm{c} 1.00, \mathrm{CH}_{3} \mathrm{CN}\right) ; \mathrm{HRMS}(\mathrm{CI}) \mathrm{m} / \mathrm{z}:[\mathrm{M}+\mathrm{H}]$ calcd for $\mathrm{C}_{11} \mathrm{H}_{18} \mathrm{~N}_{2} \mathrm{O}_{3}$ : 227.1396; found: 227.1389. 


\section{Methyl (\{2-butyl-1-[2-(9H-fluoren-9-ylmethoxycarbonylamino)-propionylamino]}

-cycloprop-2-ene carbonyl\}-amino)-acetic ester (13)

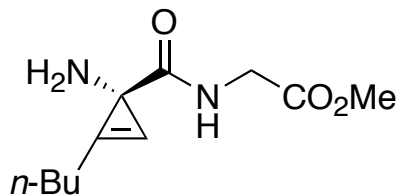

12

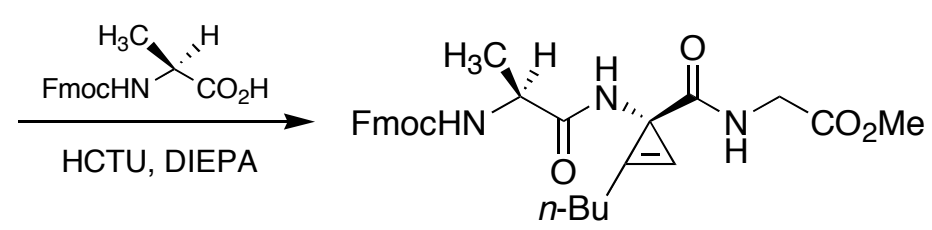

13

The procedure was identical to that used to prepare 11, except that $\mathbf{1 2}$ (34 $\mathrm{mg}, 0.15$ mmol), Fmoc-Ala-OH (47 mg, $0.15 \mathrm{mmol}$ ) was coupled. Other reagents were used in the following amounts: HCTU (74 mg, $0.18 \mathrm{mmol})$, DIPEA (58 mg, $0.45 \mathrm{mmol})$ and $\mathrm{CH}_{3} \mathrm{CN}$ (1 mL). Chromatography (10\% $\mathrm{CH}_{3} \mathrm{CN}$ in $\mathrm{CH}_{2} \mathrm{Cl}_{2}$ ) gave $62 \mathrm{mg}(80 \%)$ of the title compound as semisolid. ${ }^{1} \mathrm{H}$ NMR $\left(400 \mathrm{MHz}, \mathrm{CDCl}_{3}\right) \delta: 7.77(\mathrm{~d}, J=7.5 \mathrm{~Hz}, 2 \mathrm{H}), 7.59$ (m, 2H), 7.40 (appt, J=7.4Hz, 2H), $7.33(\mathrm{~m}, 2 \mathrm{H}), 6.88(\mathrm{~s}, 1 \mathrm{H}), 6.80$ (s, 1H), $6.72(\mathrm{~s}, 1 \mathrm{H}), 5.34$ $(\mathrm{m}, 1 \mathrm{H}), 4.42(\mathrm{~m}, 2 \mathrm{H}), 4.21(\mathrm{~m}, 1 \mathrm{H}), 4.02(\mathrm{~m}, 3 \mathrm{H}), 3.69(\mathrm{~s}, 3 \mathrm{H}), 2.58(\mathrm{t}, J=7.4 \mathrm{~Hz}, 2 \mathrm{H})$, $1.60(\mathrm{~m}, 2 \mathrm{H}), 1.39(\mathrm{~m}, 5 \mathrm{H}), 0.90(\mathrm{t}, J=7.3 \mathrm{~Hz}, 3 \mathrm{H}) ;{ }^{13} \mathrm{C} \mathrm{NMR}\left(100 \mathrm{MHz}, \mathrm{CDCl}_{3}\right) \delta: 173.5$ (u), $173.4(u), 171.0(u), 156.8(u), 144.16(u)^{*}, 144.13(u)^{*}, 141.7(u), 128.2(d n), 127.5$ (dn), $125.49(\mathrm{dn})^{*}, 125.43(\mathrm{dn})^{*}, 122.3(\mathrm{u}), 120.5(\mathrm{dn}), 100.1(\mathrm{u}), 67.6(\mathrm{u}), 52.7(\mathrm{dn})$, $51.6(\mathrm{dn}), 47.5(\mathrm{dn}), 42.1(\mathrm{u}), 39.5(\mathrm{u}), 29.3(\mathrm{u}), 24.7(\mathrm{u}), 22.8(\mathrm{u}), 18.5(\mathrm{dn}), 14.2(\mathrm{dn})$; IR $\left(\mathrm{CH}_{2} \mathrm{Cl}_{2}, \mathrm{~cm}^{-1}\right): 1747,1712,1673,1536,1449,1256,1217,1081,911,742 ;$ $[\alpha]_{\mathrm{D}}^{25}=-7.2^{\circ}\left(\mathrm{c} 1.00, \mathrm{CH}_{2} \mathrm{Cl}_{2}\right.$ ); HRMS (ESI+) $\mathrm{m} / \mathrm{z}$ : [M+Na] calcd for $\mathrm{C}_{29} \mathrm{H}_{33} \mathrm{~N}_{3} \mathrm{O}_{6}$ : 542.2267; found: 542.2293.

*Note: The observation of more then 23 peaks in the ${ }^{13} \mathrm{C}$ NMR spectrum is explained by the diastereotopic nature of the Fmoc carbons. The peaks at 125.49 and 125.43 ppm are assigned to carbons with a diastereotopic relationship. Similarly, the peaks at 144.16 and 144.13 are assigned to diastereotopic carbons. 
Methyl \{[1-(2-amino-propionylamino)-2-butyl-cycloprop-2-ene carbonyl]-amino\}acetic ester (14)

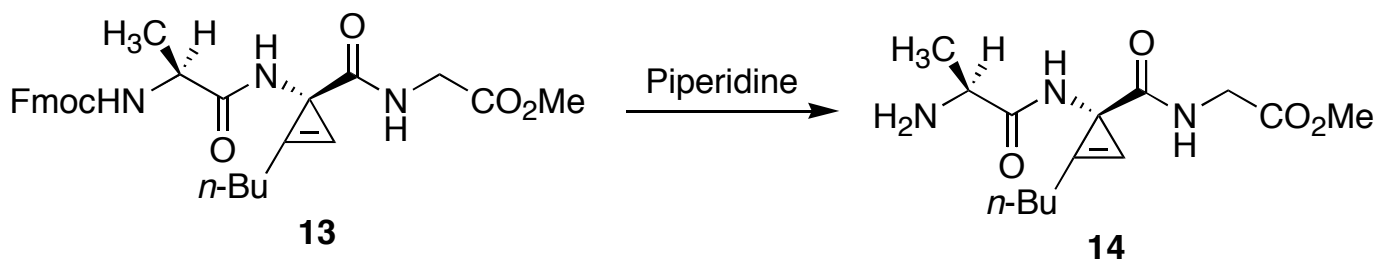

A solution of $\mathbf{1 3}(24 \mathrm{mg}, 0.05 \mathrm{mmol})$ in $20 \%$ piperidine in $\mathrm{CH}_{2} \mathrm{Cl}_{2}$ was allowed to stir for $10 \mathrm{~min}$ at $\mathrm{rt}$. The reaction mixture was then concentrated and chromatographed (gradient of $10 \%-30 \% \mathrm{MeOH}$ in $\mathrm{CH}_{2} \mathrm{Cl}_{2}$ ) to give $15 \mathrm{mg}$ (100\%) of the title compound as semisolid. ${ }^{1} \mathrm{H}$ NMR $\left(400 \mathrm{MHz}, \mathrm{CD}_{3} \mathrm{CN}\right) \delta: 7.81(\mathrm{bs}, 1 \mathrm{H}), 6.81(\mathrm{~m}, 1 \mathrm{H}), 6.77(\mathrm{~s}, 1 \mathrm{H}), 3.86(\mathrm{~m}, 2 \mathrm{H})$, $3.65(\mathrm{~s}, 3 \mathrm{H}), 3.50(\mathrm{~m}, 1 \mathrm{H}), 2.53(\mathrm{~m}, 2 \mathrm{H}), 1.57(\mathrm{~m}, 2 \mathrm{H}), 1.39(\mathrm{~m}, 2 \mathrm{H}), 1.24(\mathrm{~d}, J=6.6 \mathrm{~Hz}$, 3H), $0.90(\mathrm{t}, J=7.3 \mathrm{~Hz}, 3 \mathrm{H}) ;{ }^{13} \mathrm{C} \mathrm{NMR}\left(100 \mathrm{MHz}, \mathrm{CDCl}_{3}\right) \delta: 177.3$ (u), 173.7 (u), 171.0 (u), $122.7(u), 100.7(u), 52.8(d n), 51.4(d n), 42.1(u), 39.0(u), 29.3(u), 24.8(u), 22.8$ (u), $22.1(\mathrm{dn}), 14.2(\mathrm{dn})$; IR $\left(\mathrm{CHCl}_{3}, \mathrm{~cm}^{-1}\right): 3360,1756,1660,1650,1516,1256,1208$, 1182, 906, 735; $[\alpha]_{\mathrm{D}}{ }^{25}=-16.5^{\circ}\left(\mathrm{c} 1.00, \mathrm{CHCl}_{3}\right) ; \mathrm{HRMS}(\mathrm{ESI}+) \mathrm{m} / \mathrm{z}:[\mathrm{M}+\mathrm{H}]$ calcd for $\mathrm{C}_{14} \mathrm{H}_{23} \mathrm{~N}_{3} \mathrm{O}_{4}$ : 298.1767; found: 298.1773. 
${ }^{1} \mathrm{H}$ NMR spectrum of dimethyl 2-isopropyl-cycloprop-2-ene-1,1-dicarboxylic ester (400 MHz, $\mathrm{CDCl}_{3}$ )

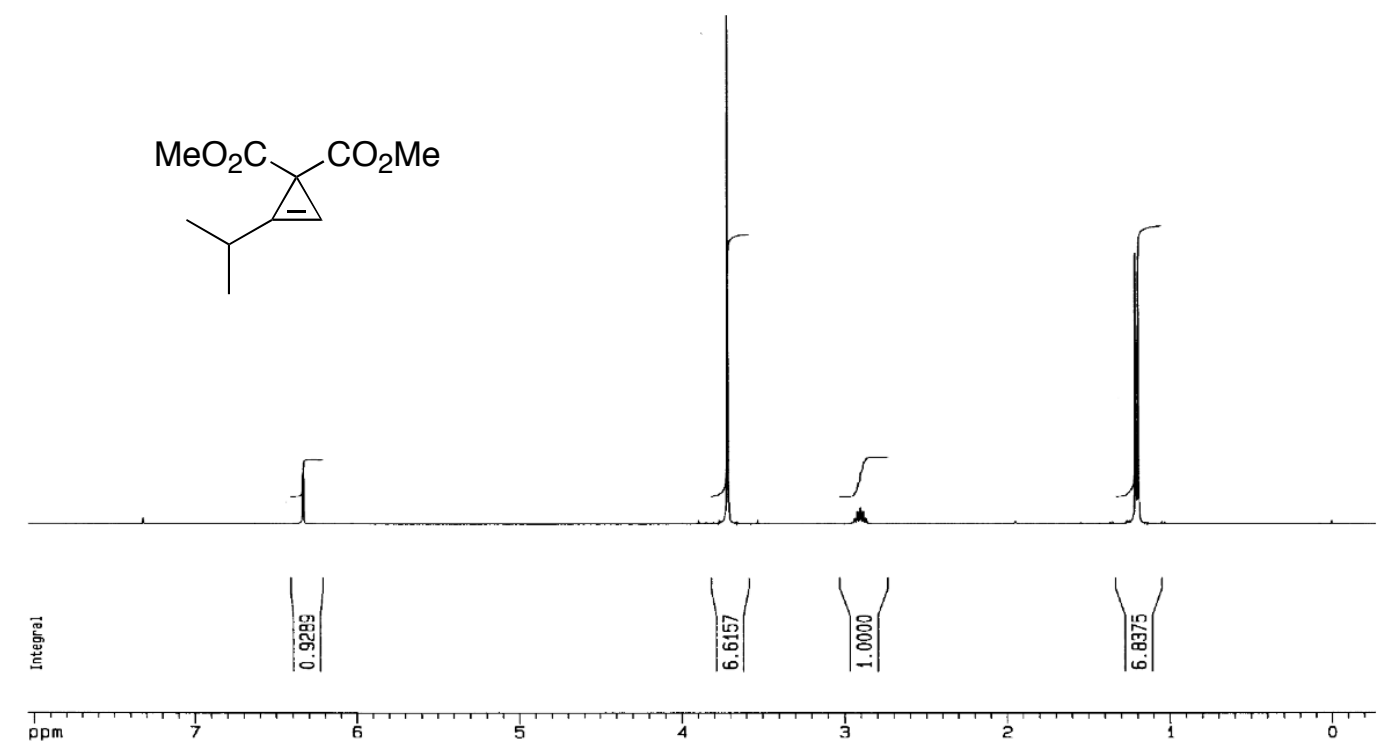

${ }^{13} \mathrm{C}$ NMR spectrum of dimethyl 2-isopropyl-cycloprop-2-ene-1,1-dicarboxylic ester $\left(100 \mathrm{MHz}, \mathrm{CDCl}_{3}\right.$ )

\begin{tabular}{|c|c|c|}
\hline & 요 & 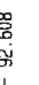 \\
\hline
\end{tabular}

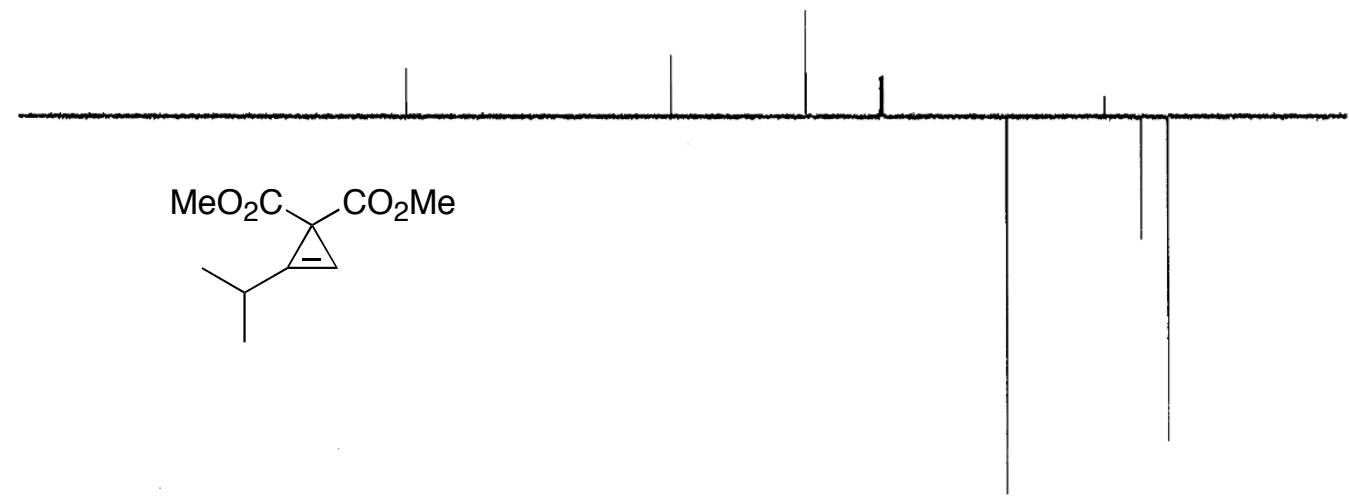

ppm

200

150

100

50

ग गमा1' 
${ }^{1} \mathrm{H}$ NMR spectrum of dimethyl 2-(4-fluorophenyl)-cycloprop-2-ene-1,1-dicarboxylic ester $\left(400 \mathrm{MHz}, \mathrm{CDCl}_{3}\right)$
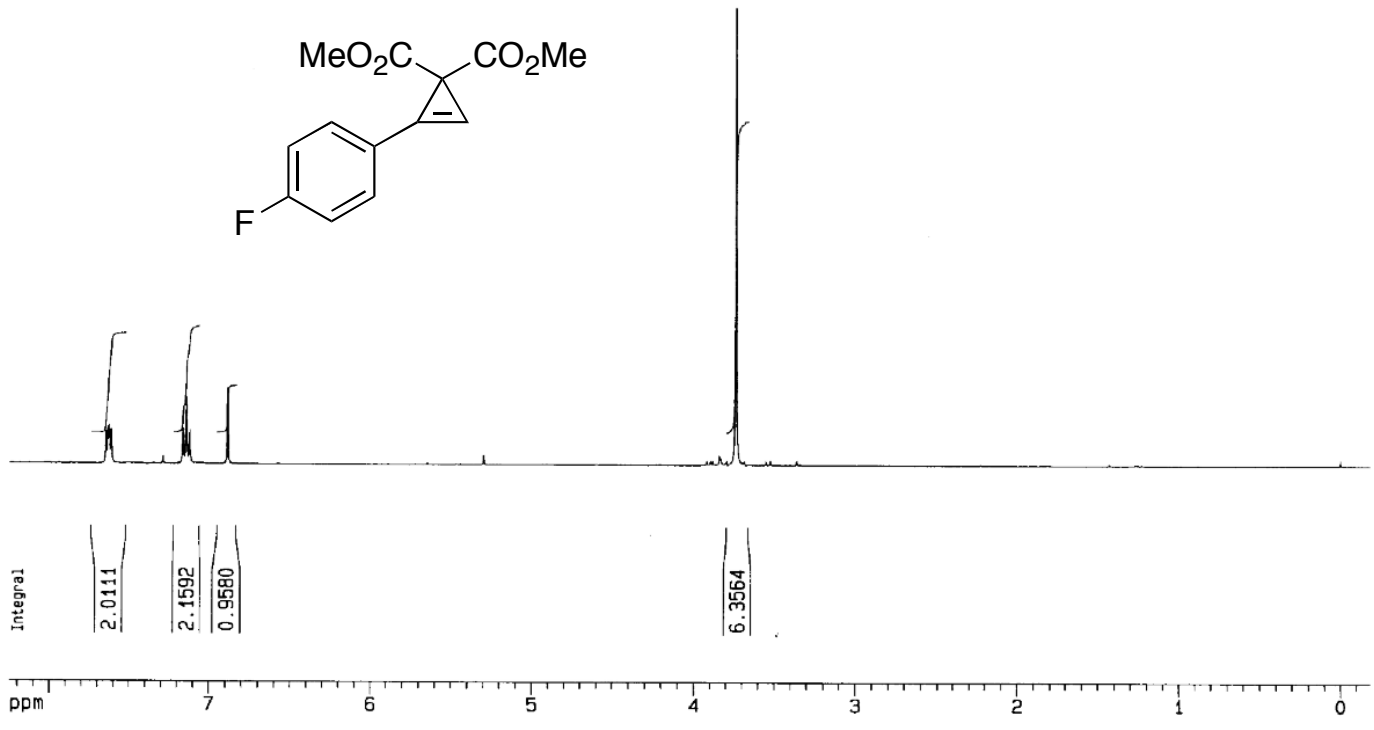

${ }^{13} \mathrm{C}$ NMR spectrum of dimethyl 2-(4-fluorophenyl)-cycloprop-2-ene-1,1-dicarboxylic ester $\left(90 \mathrm{MHz}, \mathrm{CDCl}_{3}\right)$

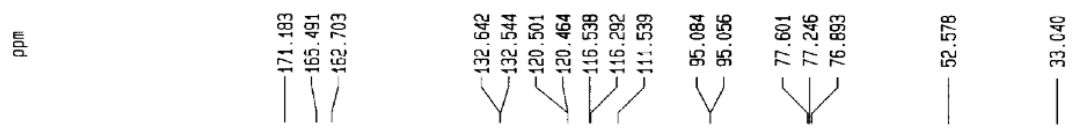

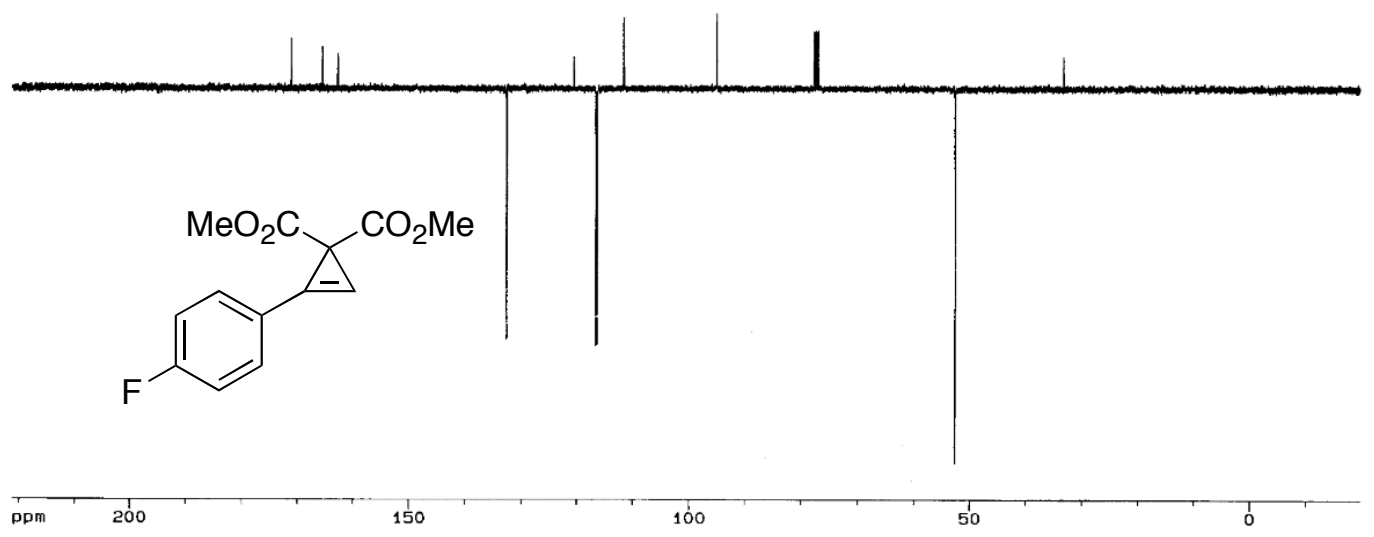


${ }^{1} \mathrm{H}$ NMR spectrum of dimethyl 2-isobutyl-cycloprop-2-ene-1,1-dicarboxylic ester $\left(400 \mathrm{MHz}, \mathrm{CDCl}_{3}\right)$

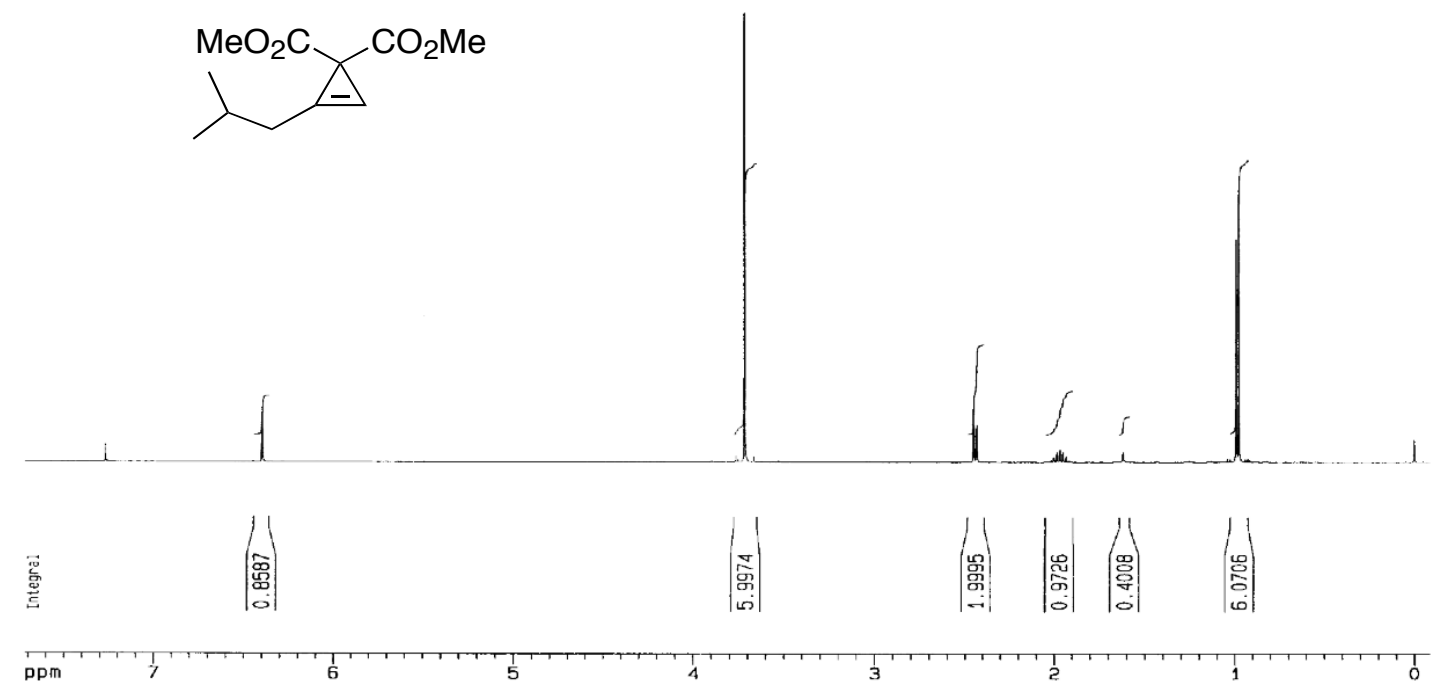

${ }^{13} \mathrm{C}$ NMR spectrum of dimethyl 2-isobutyl-cycloprop-2-ene-1,1-dicarboxylic ester $\left(100 \mathrm{MHz}, \mathrm{CDCl}_{3}\right.$ )

言
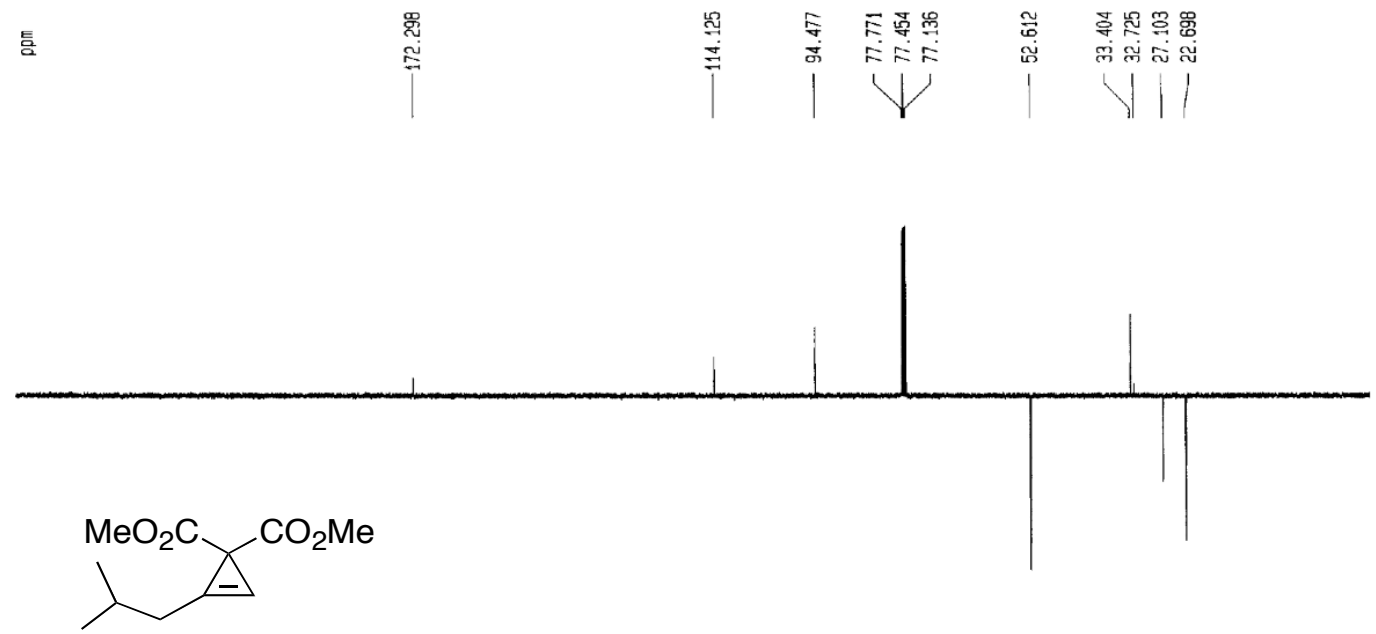

ppm

200

100

50 
${ }^{1} \mathrm{H}$ NMR spectrum of dimethyl 2-methoxymethyl-cycloprop-2-ene-1,1-dicarboxylic ester $\left(400 \mathrm{MHz}, \mathrm{CDCl}_{3}\right)$

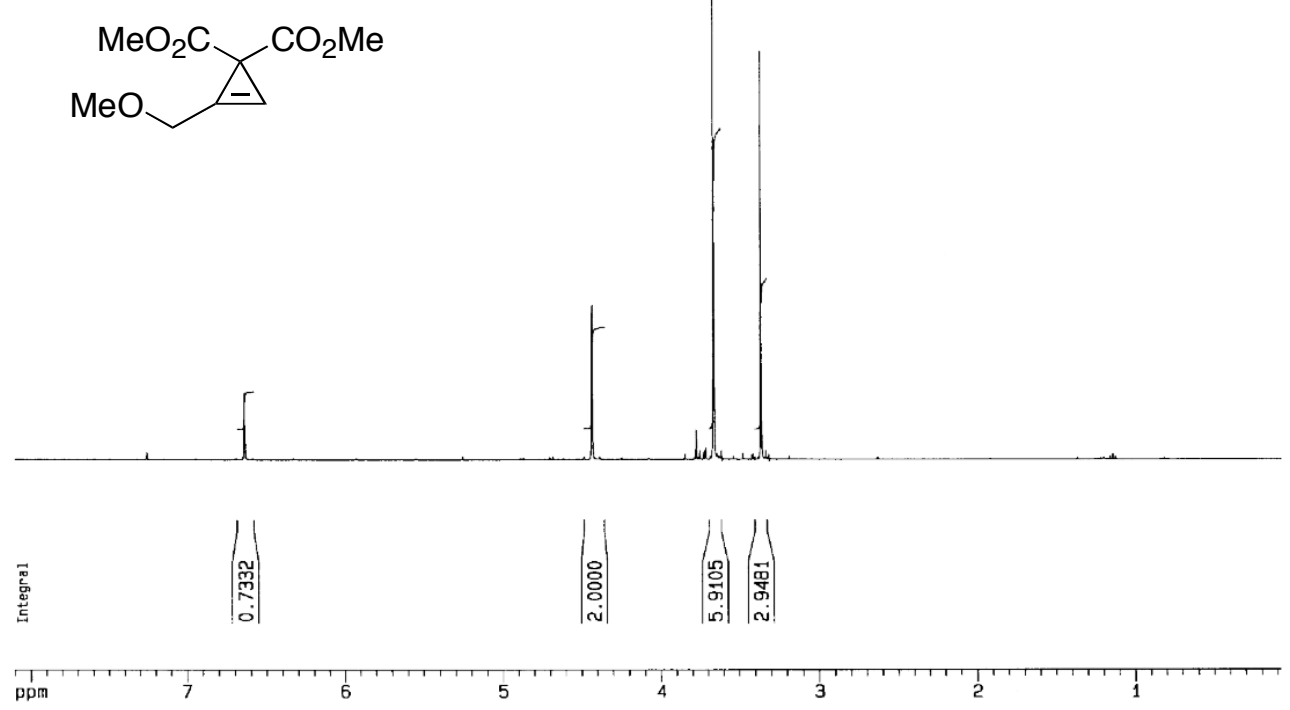

${ }^{13} \mathrm{C}$ NMR spectrum of dimethyl 2-methoxymethyl-cycloprop-2-ene-1,1-dicarboxylic ester $\left(100 \mathrm{MHz}, \mathrm{CDCl}_{3}\right)$

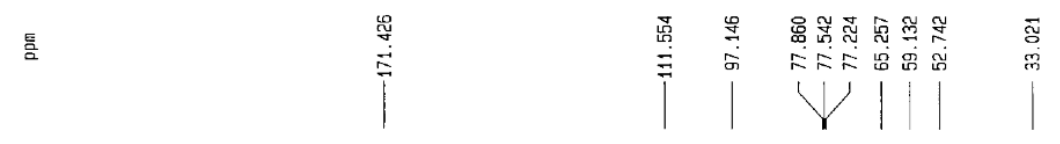
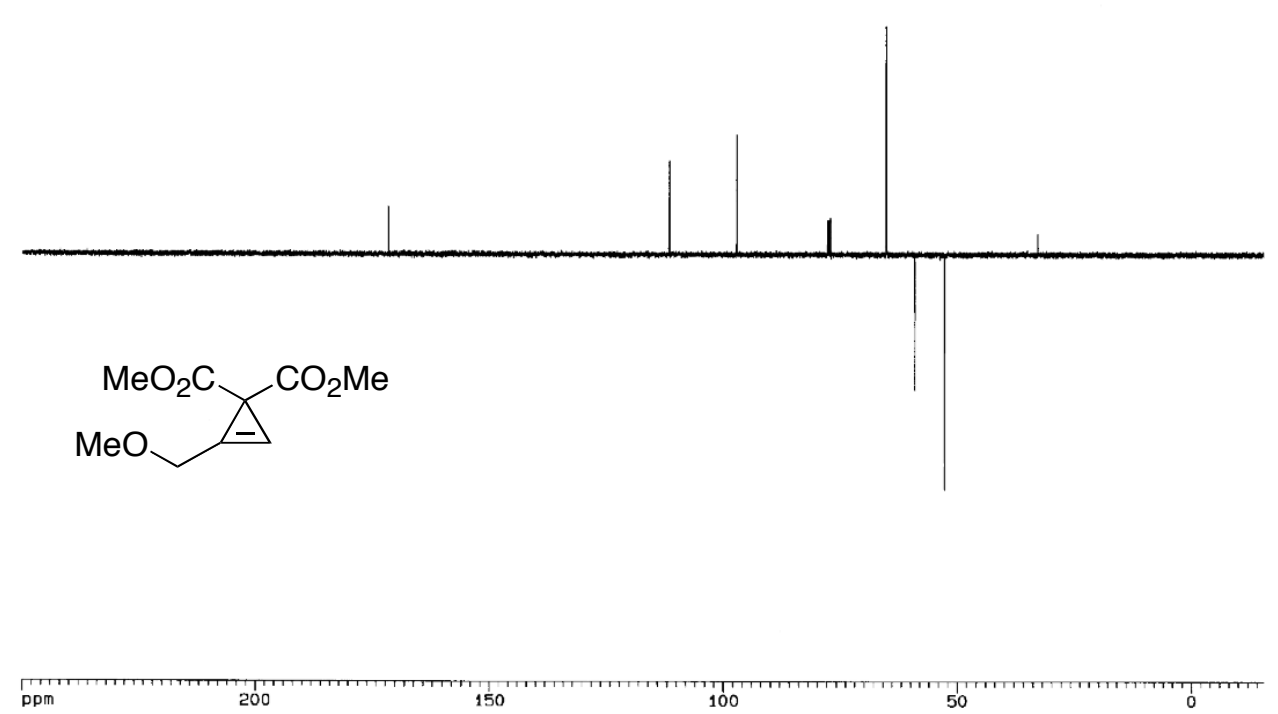
${ }^{1} \mathrm{H}$ NMR spectrum of dimethyl 2-trityloxymethyl-cycloprop-2-ene-1,1-dicarboxylic ester $\left(400 \mathrm{MHz}, \mathrm{CDCl}_{3}\right)$

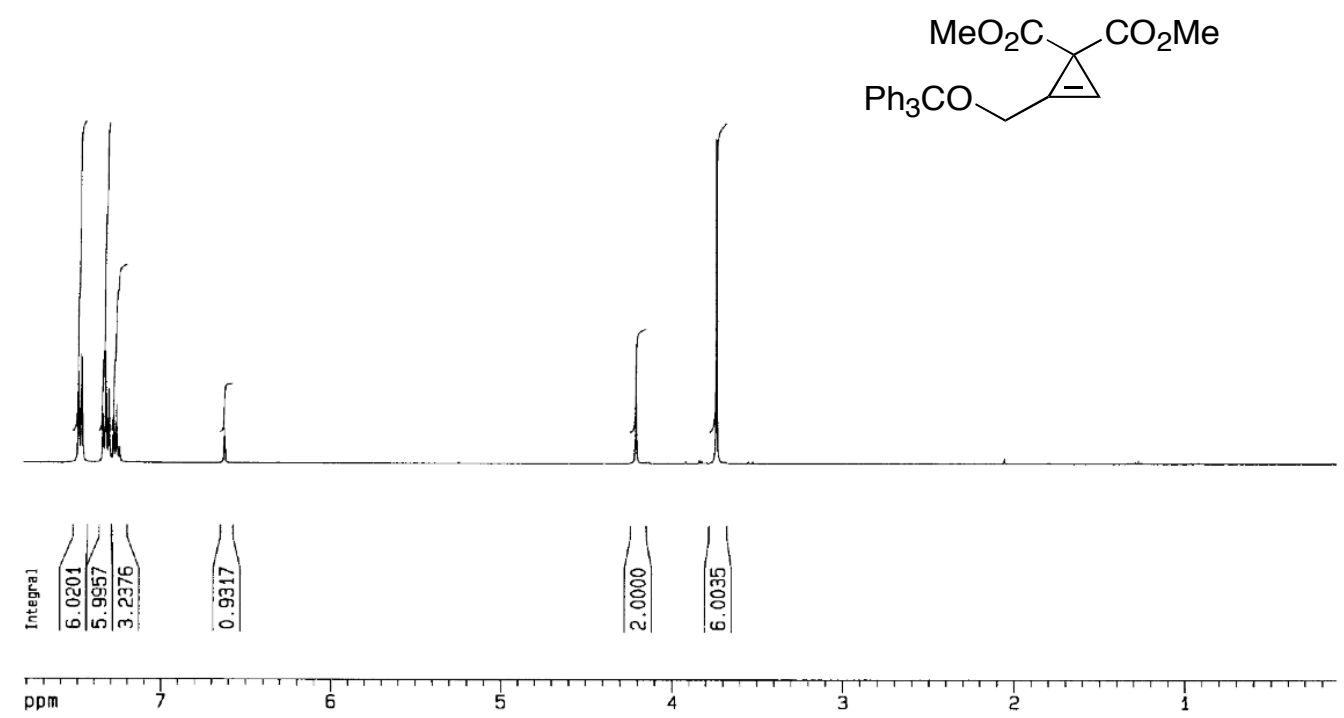

${ }^{13} \mathrm{C}$ NMR spectrum of dimethyl 2-trityloxymethyl-cycloprop-2-ene-1,1-dicarboxylic ester $\left(100 \mathrm{MHz}, \mathrm{CDCl}_{3}\right)$

镸

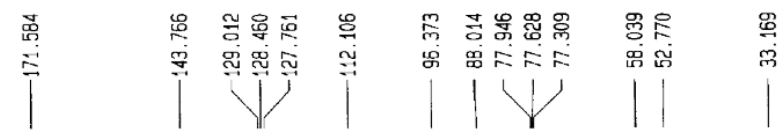

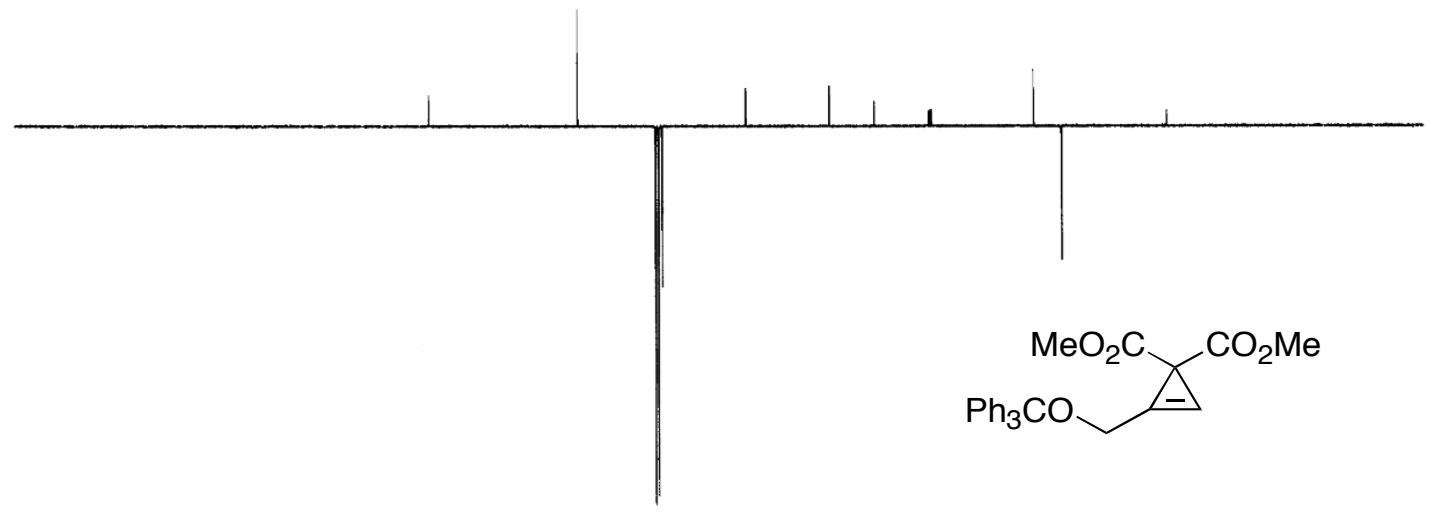


${ }^{1} \mathrm{H}$ NMR spectrum of $\mathbf{1 a}\left(400 \mathrm{MHz}, \mathrm{CD}_{3} \mathrm{CN}\right)$

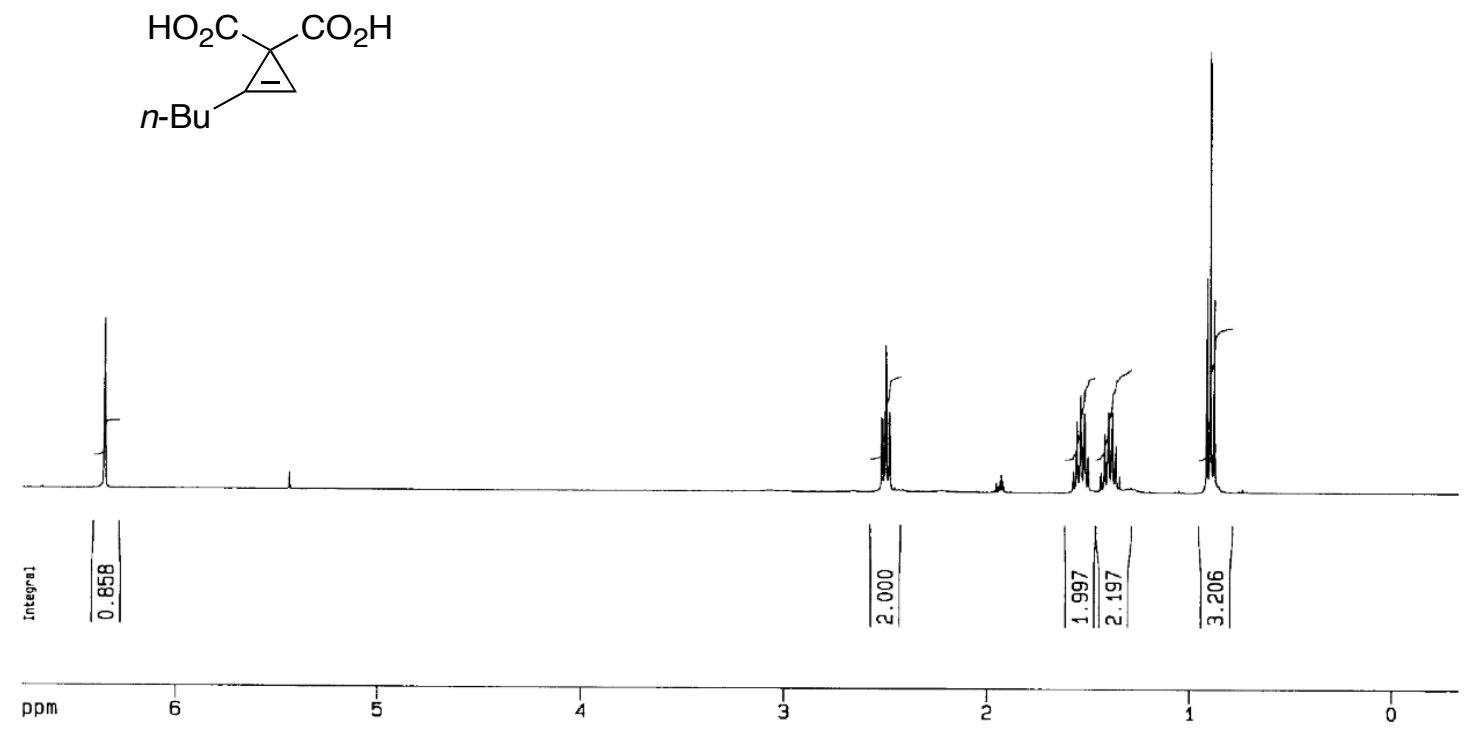

${ }^{13} \mathrm{C}$ NMR spectrum of $\mathbf{1 a}\left(90 \mathrm{MHz}, \mathrm{CD}_{3} \mathrm{CN}\right)$

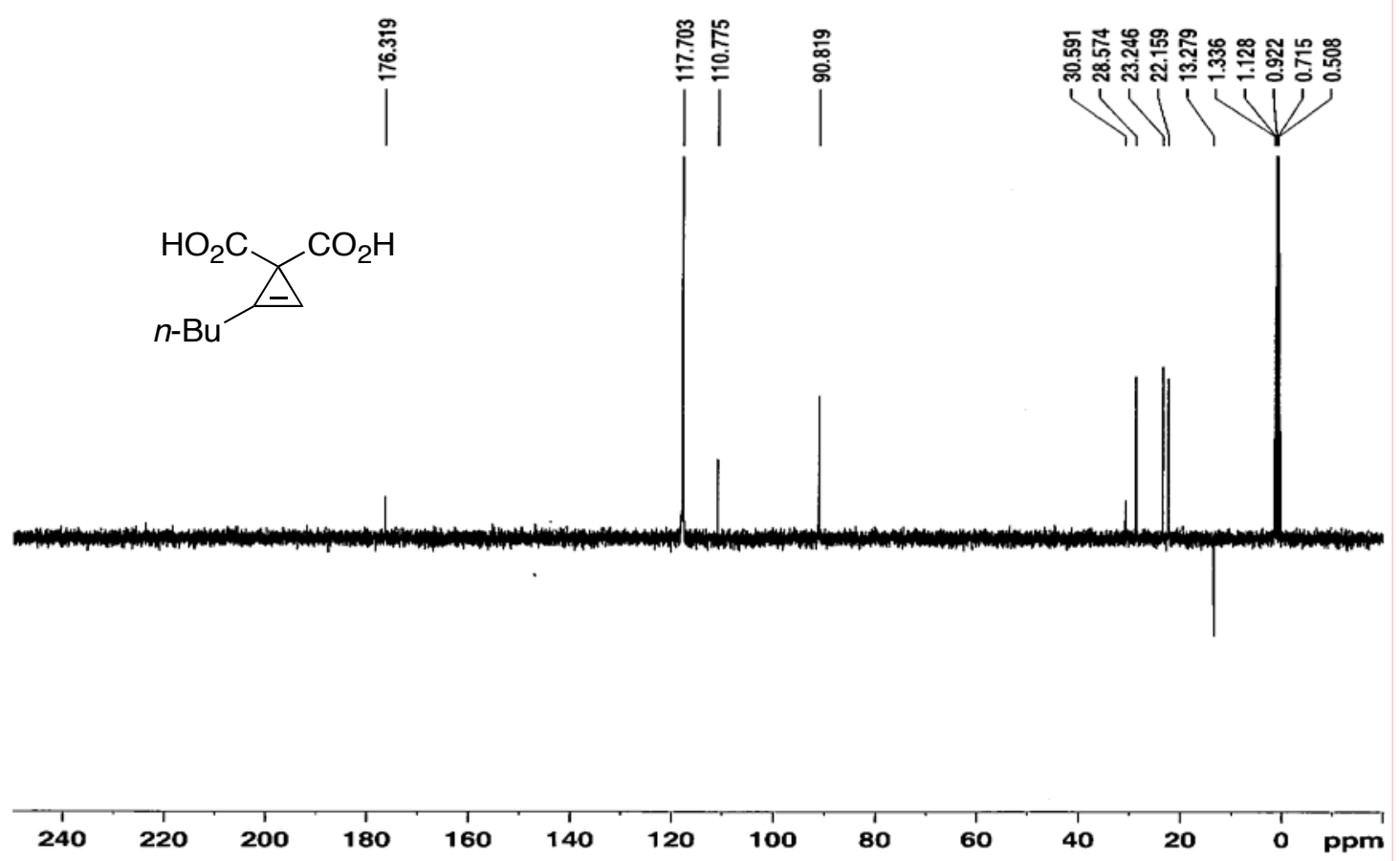


${ }^{1} \mathrm{H}$ NMR spectrum of $\mathbf{1 b}\left(400 \mathrm{MHz}, \mathrm{CD}_{3} \mathrm{CN}\right)$

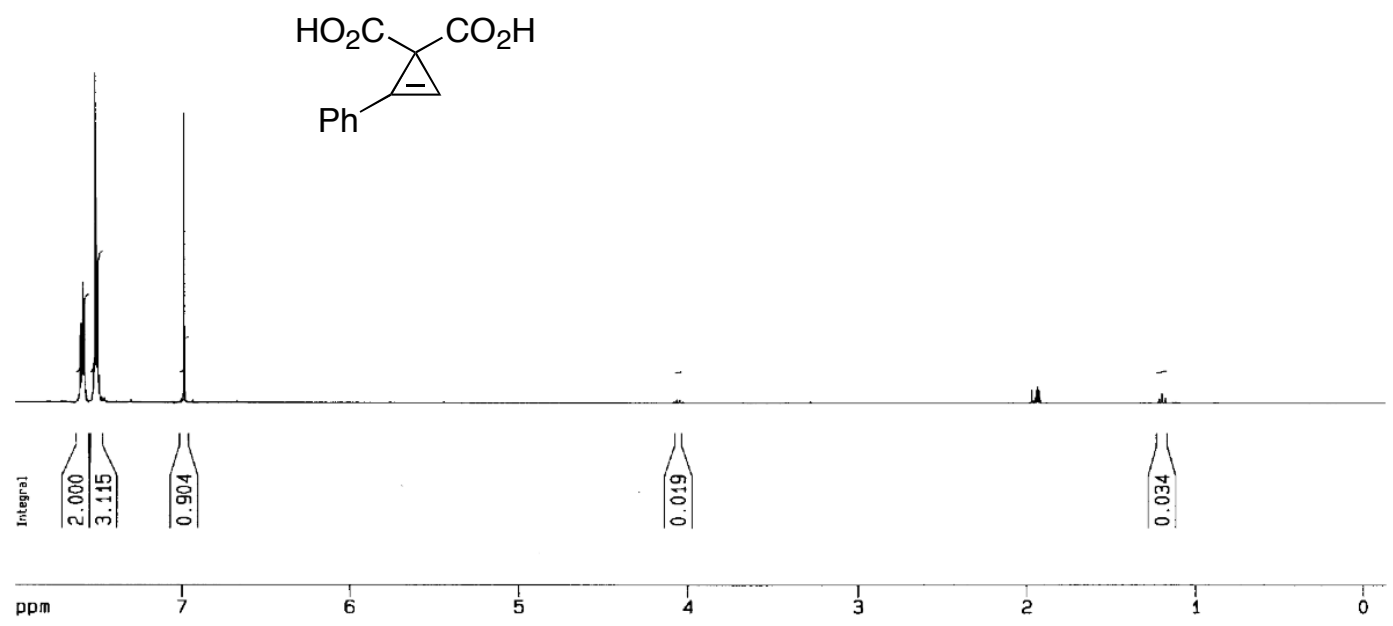

${ }^{13} \mathrm{C}$ NMR spectrum of $\mathbf{1 b}\left(100 \mathrm{MHz}, \mathrm{CD}_{3} \mathrm{CN}\right)$

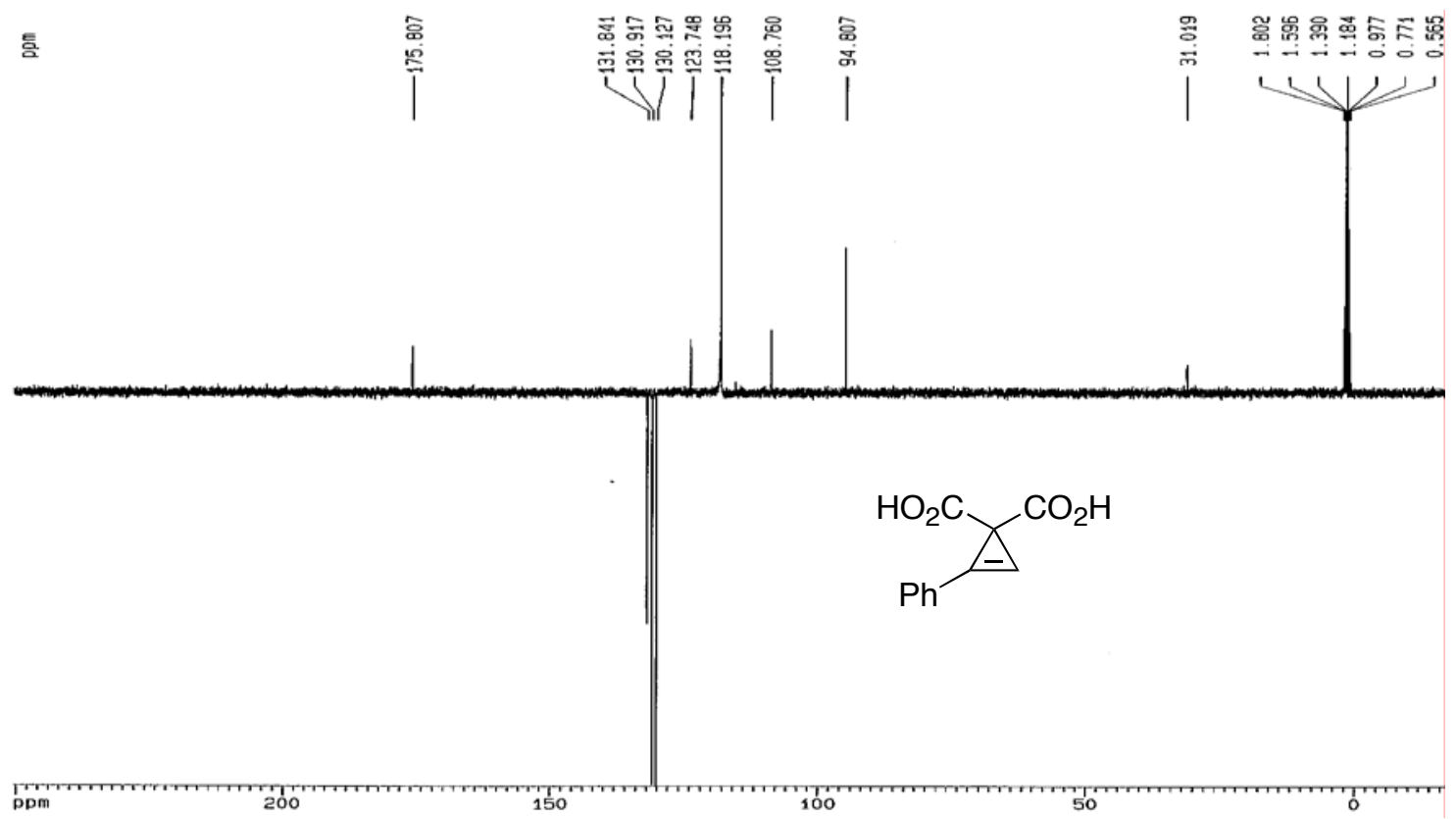


${ }^{1} \mathrm{H}$ NMR spectrum of $1 \mathrm{c}\left(400 \mathrm{MHz}, \mathrm{CD}_{3} \mathrm{CN}\right)$

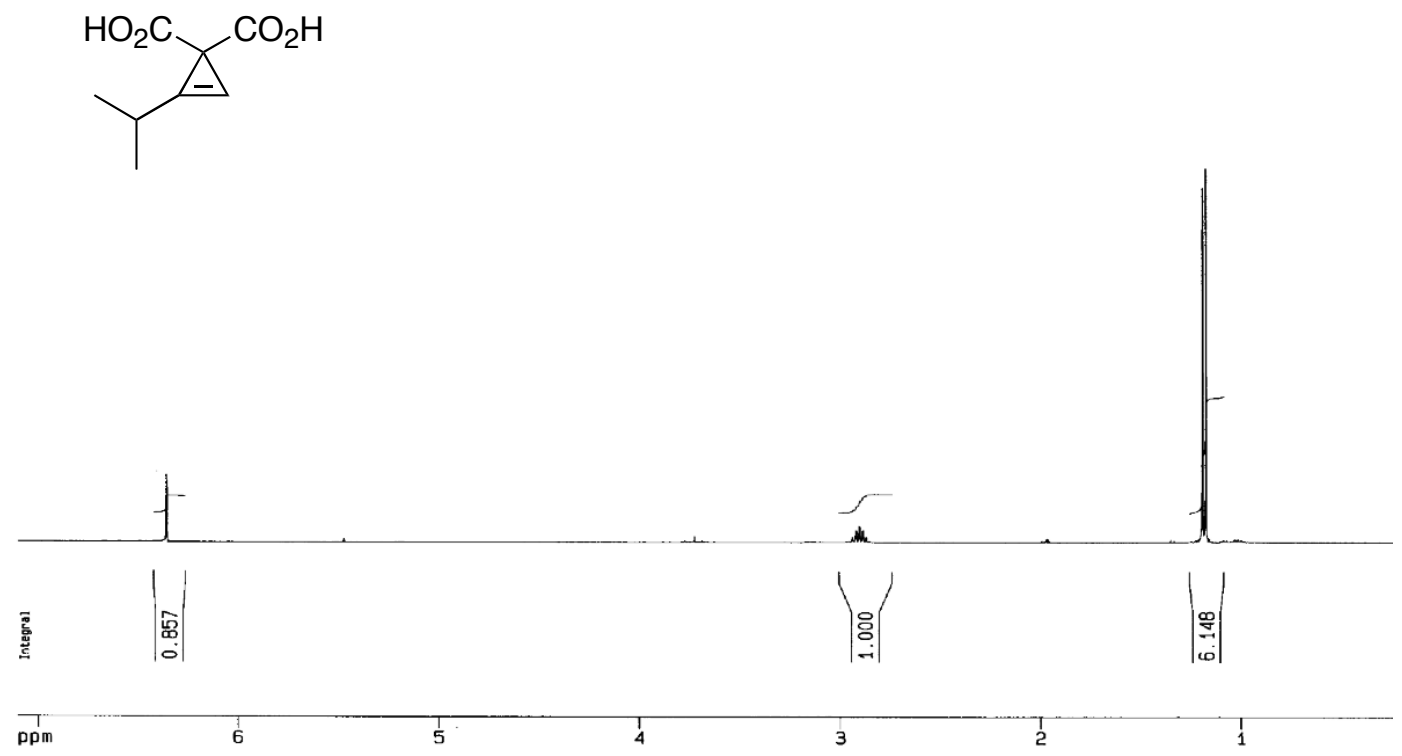

${ }^{13} \mathrm{C}$ NMR spectrum of $1 \mathrm{c}\left(100 \mathrm{MHz}, \mathrm{CD}_{3} \mathrm{CN}\right)$

틈
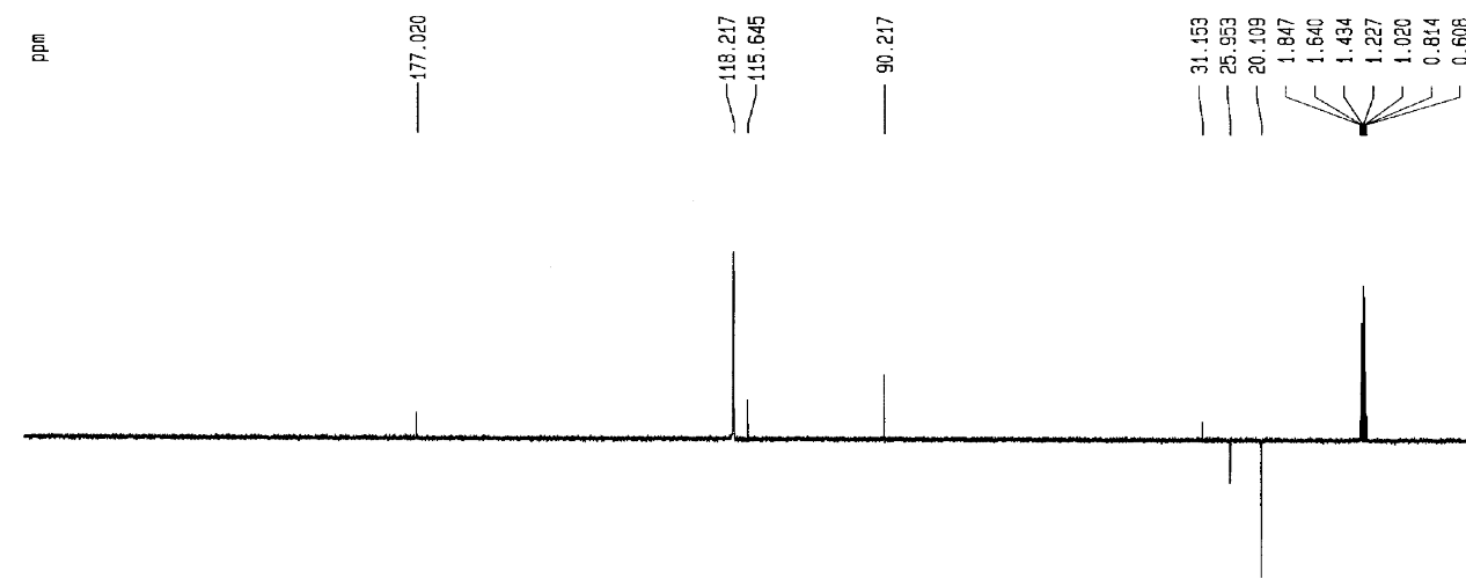

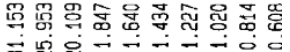
ल

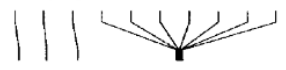


${ }^{1} \mathrm{H}$ NMR spectrum of $\mathbf{1 d}(400 \mathrm{MHz}$, acetone-d6)

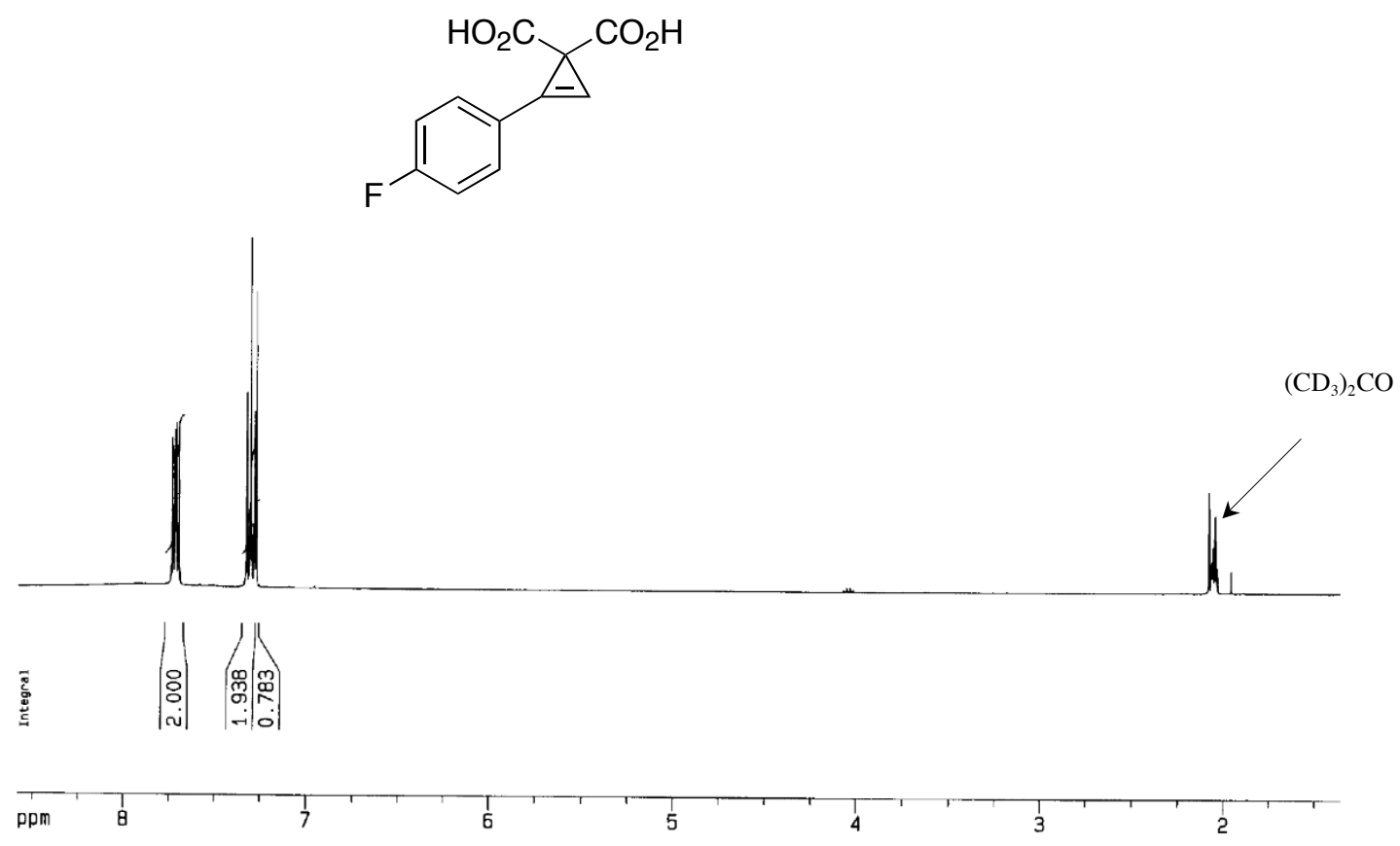

${ }^{15} \mathrm{C}$ NMR spectrum of $\mathbf{1 d}(100 \mathrm{MHz}$, acetone- $d 6)$
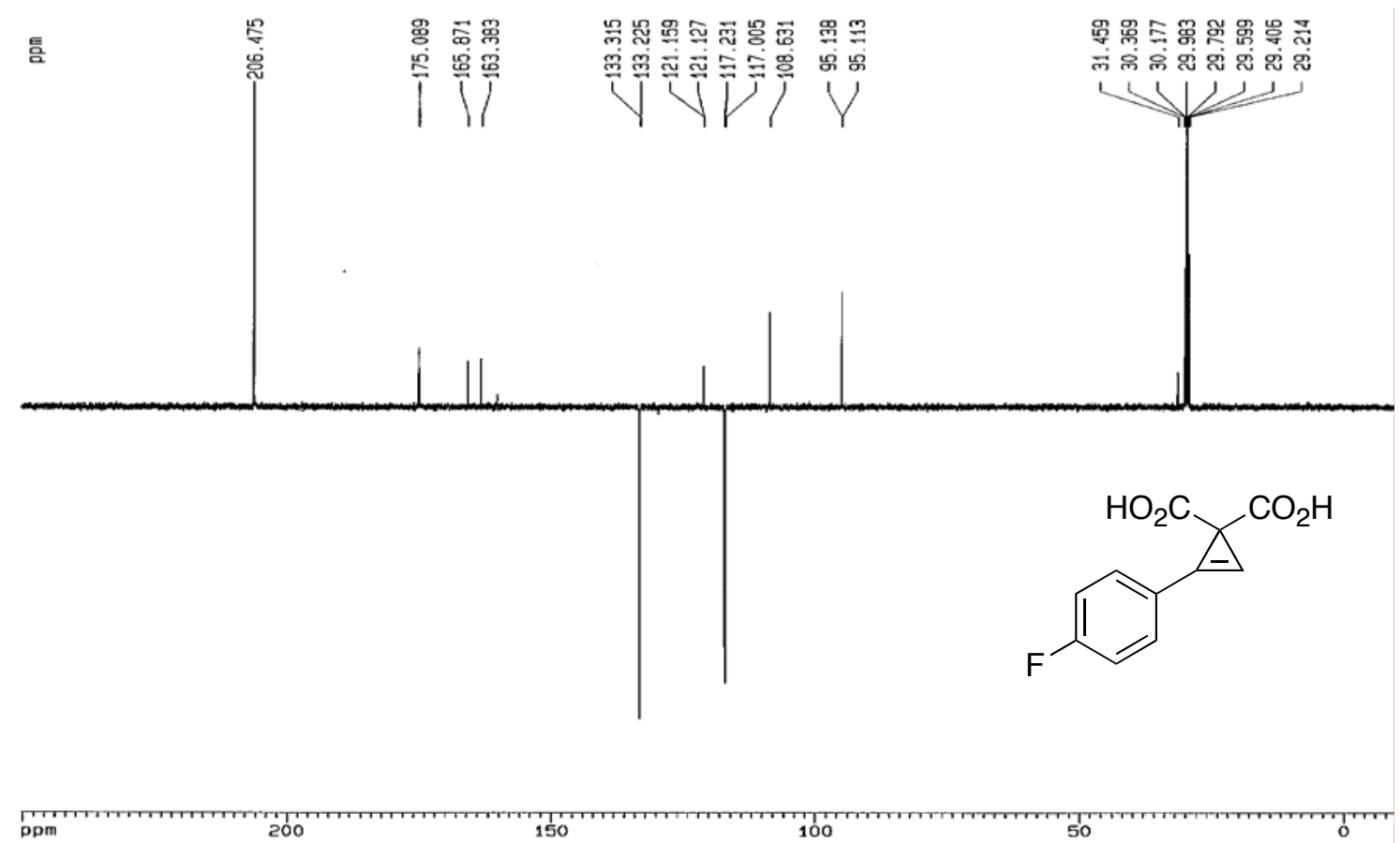
${ }^{1} \mathrm{H}$ NMR spectrum of $1 \mathrm{e}$ (400 MHz, acetone-d6)
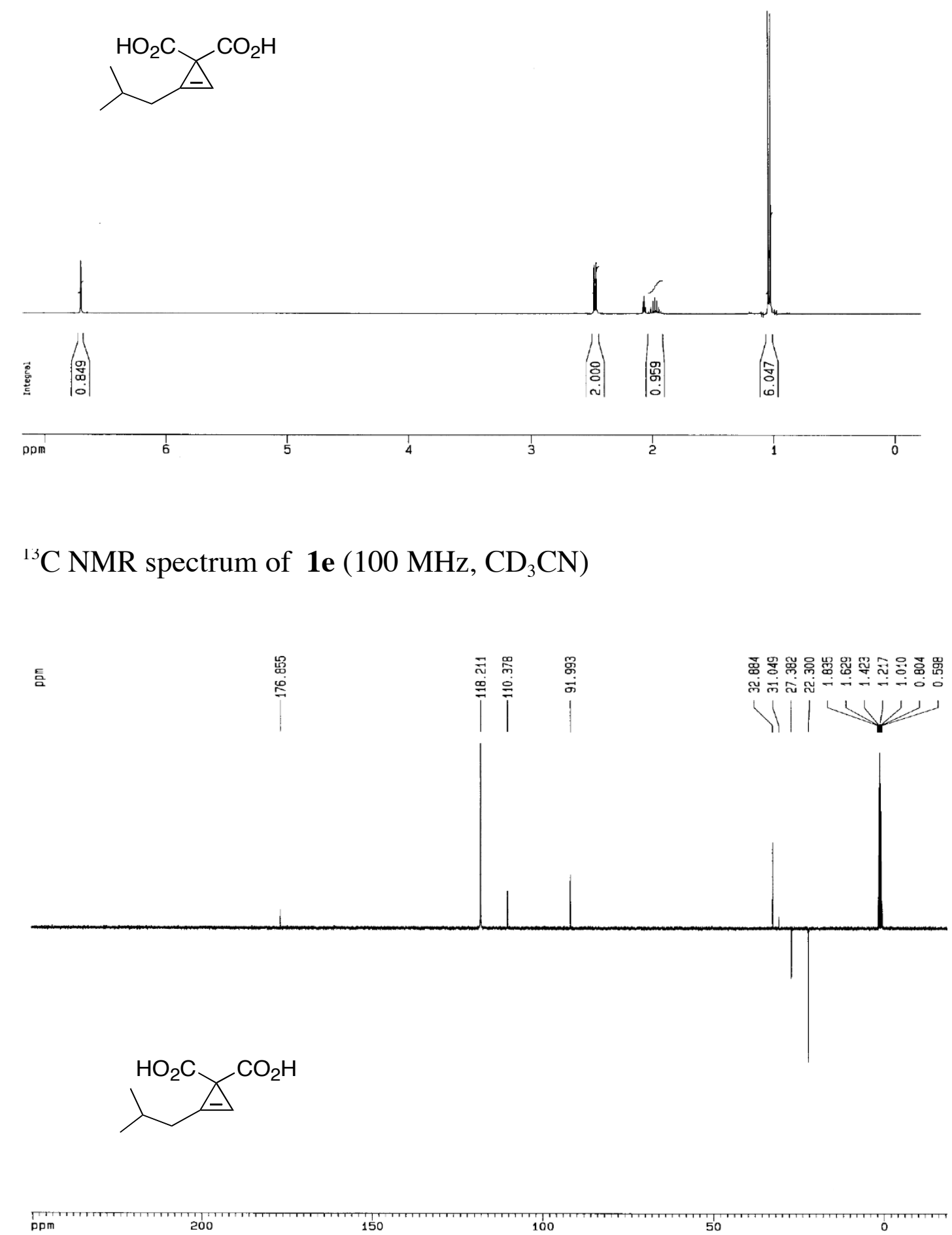
${ }^{1} \mathrm{H} \mathrm{NMR}$ spectrum of $\mathbf{1 f}\left(400 \mathrm{MHz}, \mathrm{CD}_{3} \mathrm{CN}\right)$

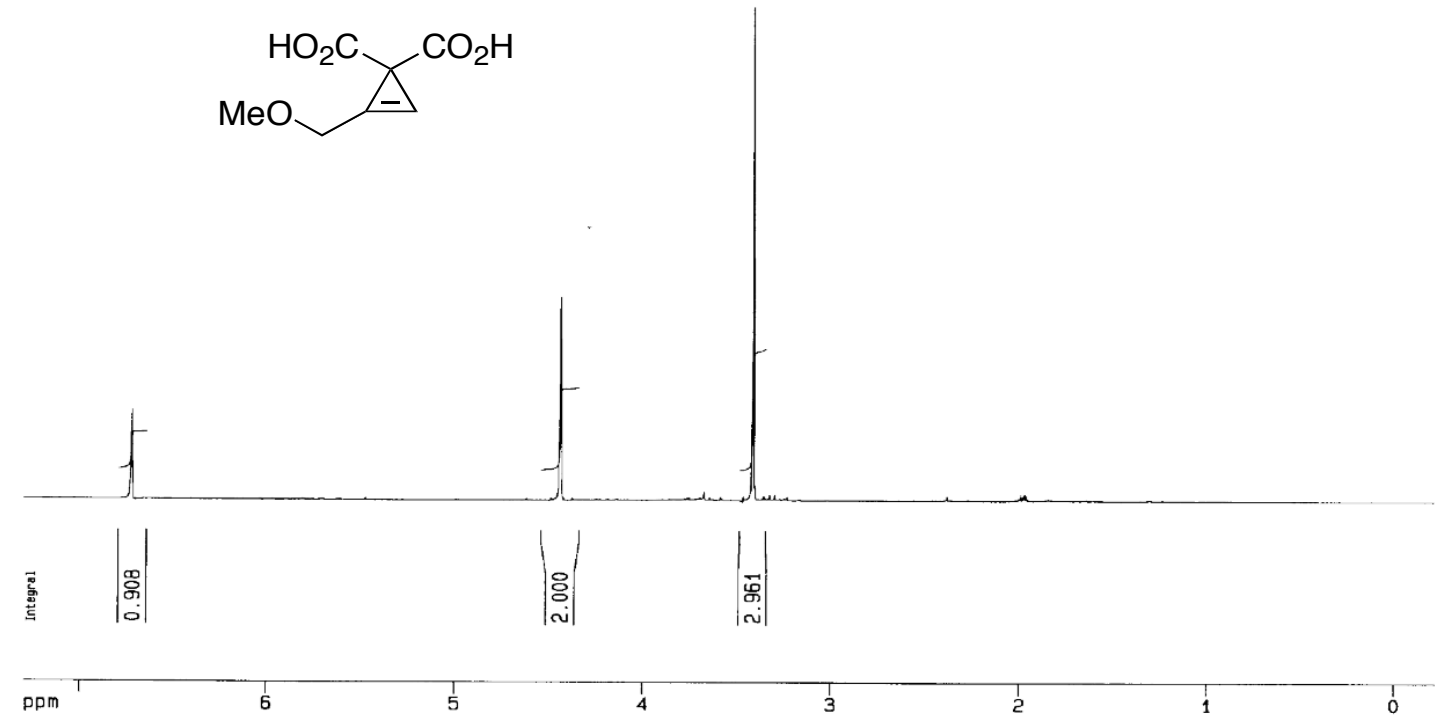

${ }^{13} \mathrm{C}$ NMR spectrum of $\mathbf{1 f}\left(90 \mathrm{MHz}, \mathrm{CD}_{3} \mathrm{CN}\right)$

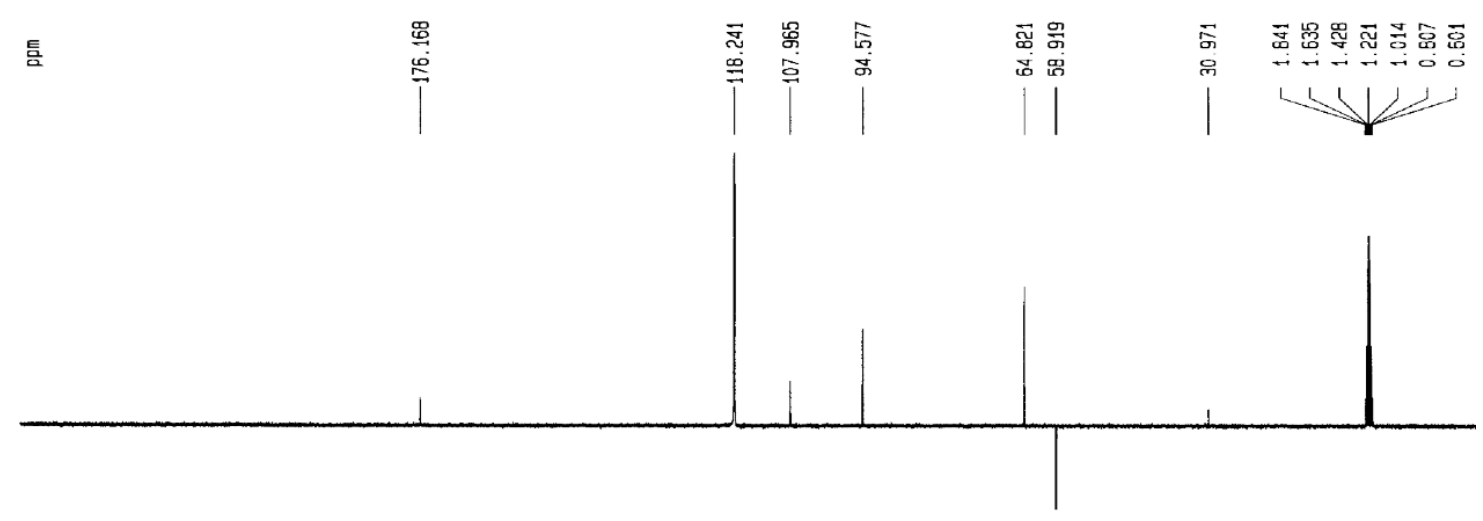
${ }_{\mathrm{MeO}}^{\mathrm{HO}_{2} \mathrm{C}} \underbrace{\mathrm{CO}_{2} \mathrm{H}}$

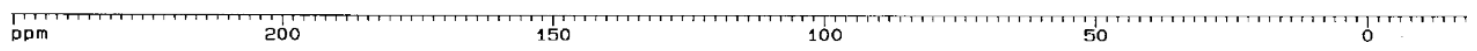


${ }^{1} \mathrm{H}$ NMR spectrum of $\mathbf{1 g}$ (400 MHz, THF- $d 8$ )

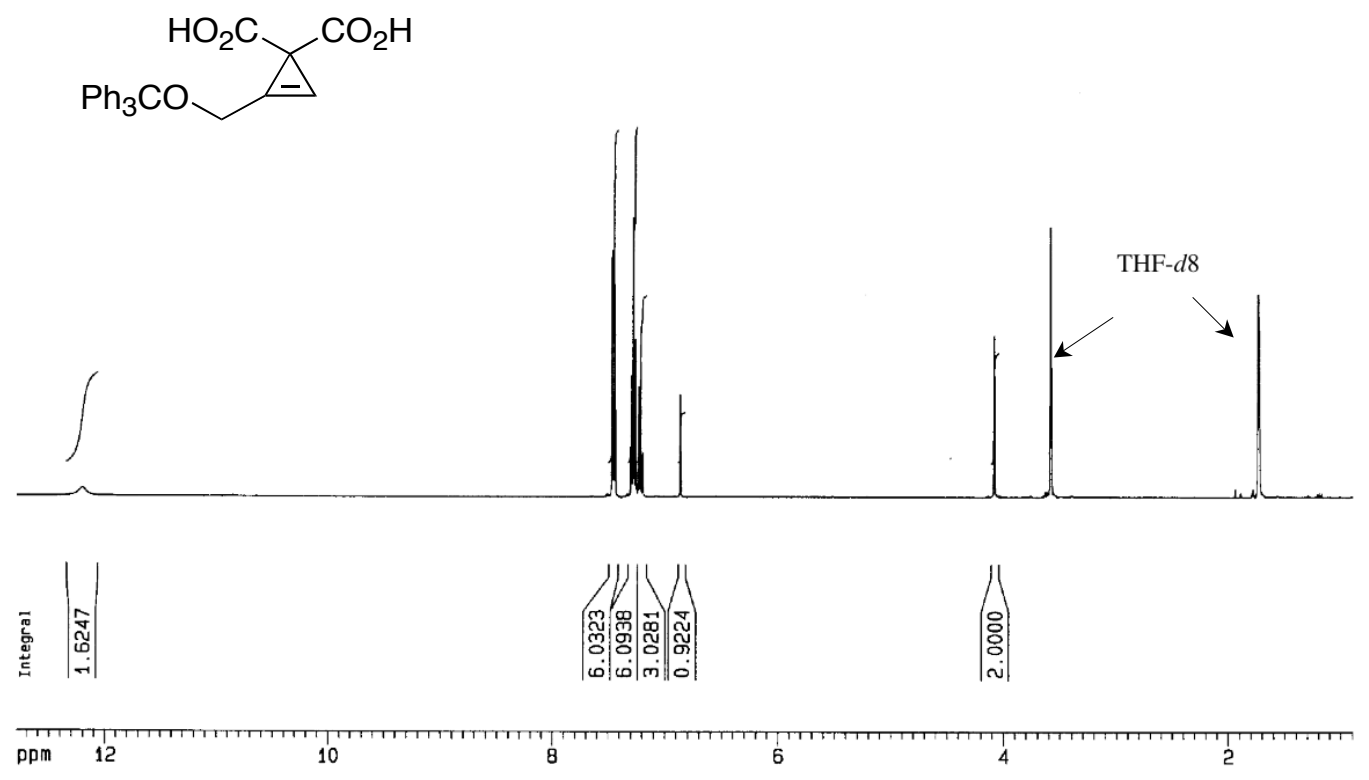

${ }^{15} \mathrm{C}$ NMR spectrum of $\mathbf{1 g}(100 \mathrm{MHz}, \mathrm{THF}-d 8)$
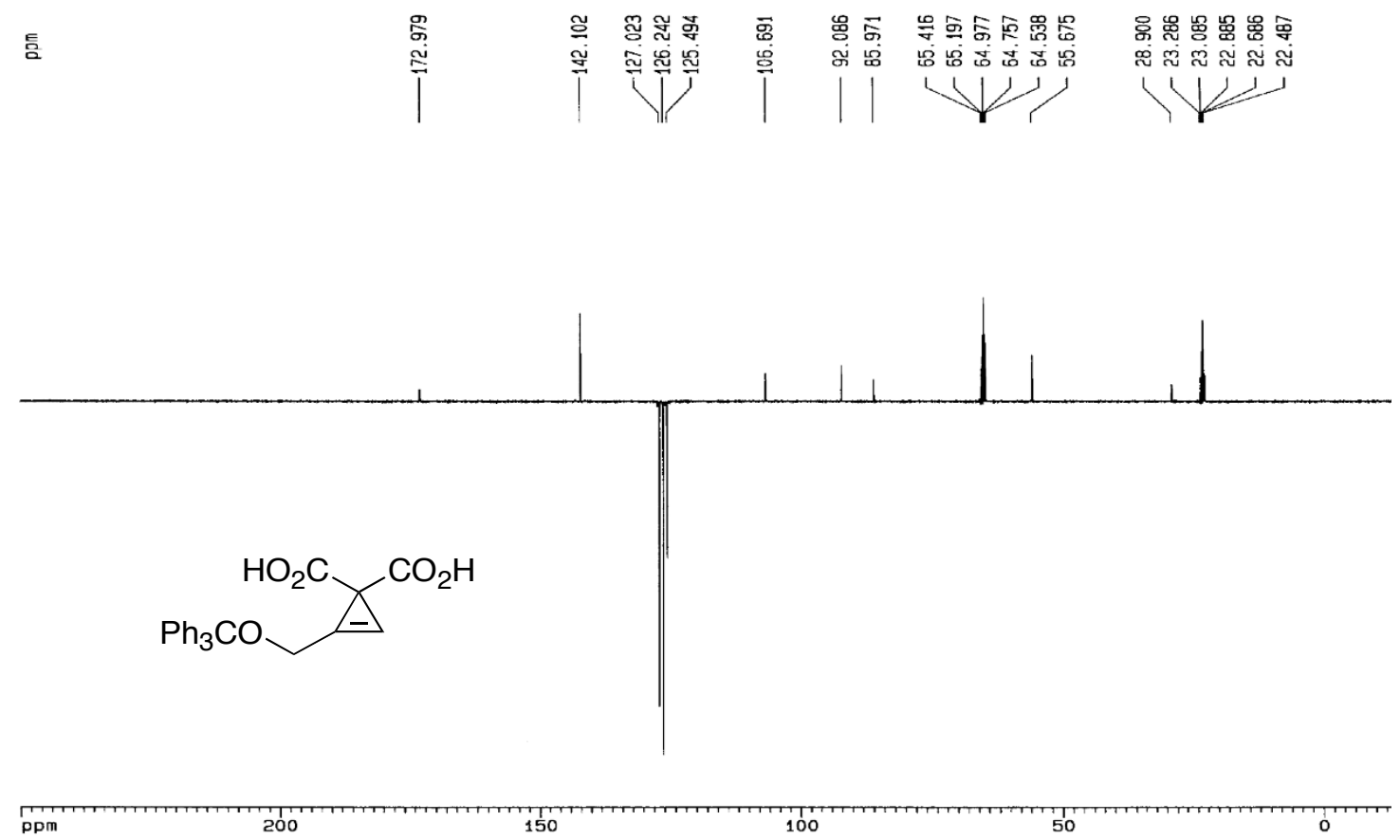
${ }^{1} \mathrm{H}$ NMR spectrum of $\mathbf{4 a}\left(400 \mathrm{MHz}, \mathrm{CDCl}_{3}\right)$

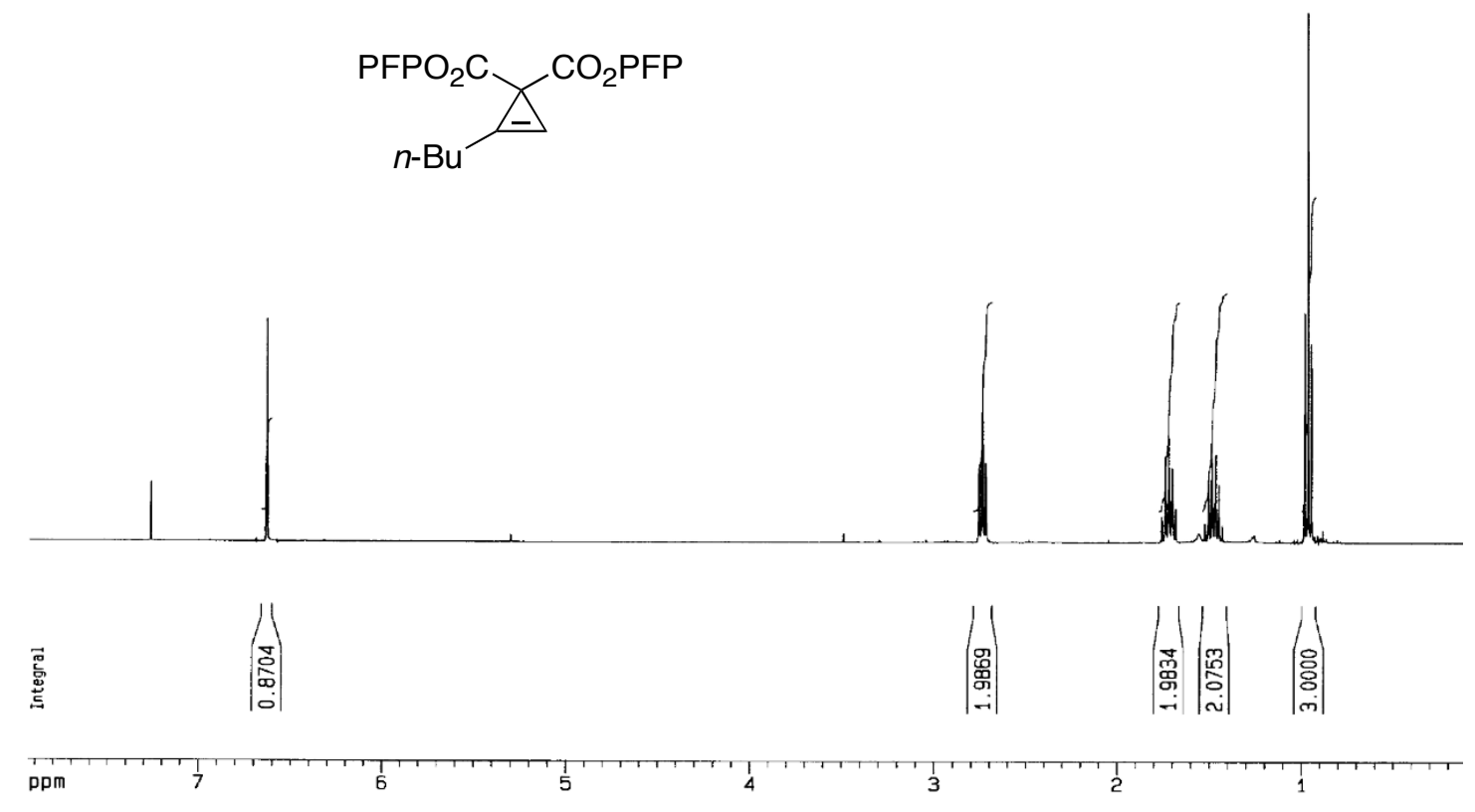

${ }^{15} \mathrm{C}$ NMR spectrum of $\mathbf{4 a}\left(100 \mathrm{MHz}, \mathrm{CDCl}_{3}\right)$

镸

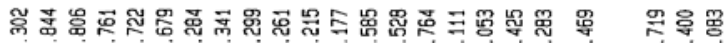

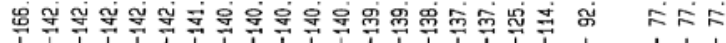

V

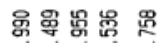

लं ฐึณ

11/
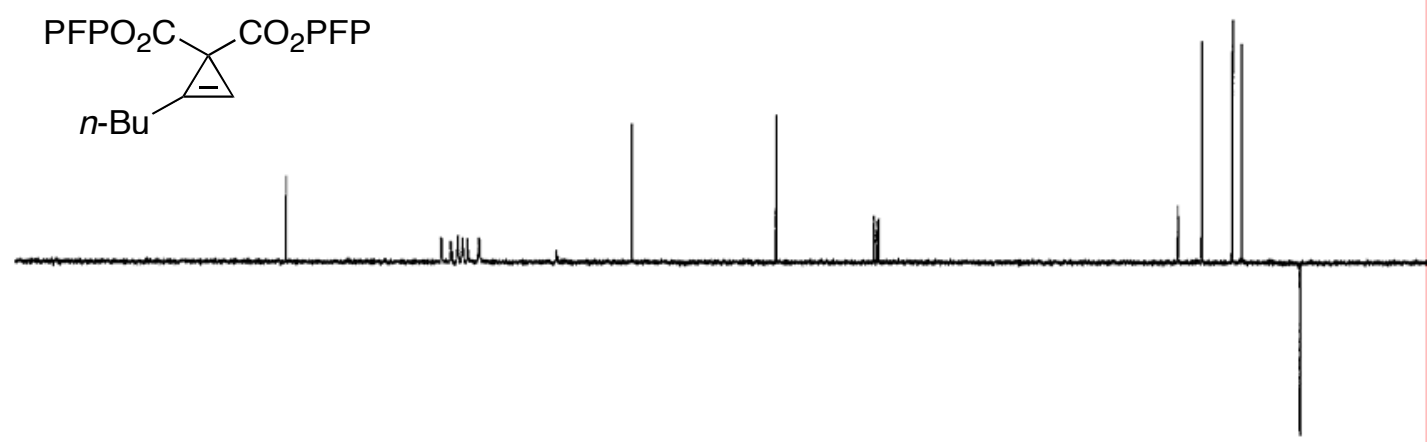

ppm

150

100

50

pre 
${ }^{1} \mathrm{H}$ NMR spectrum of $\mathbf{4 b}\left(400 \mathrm{MHz}, \mathrm{CDCl}_{3}, \sim 90 \%\right.$ purity)

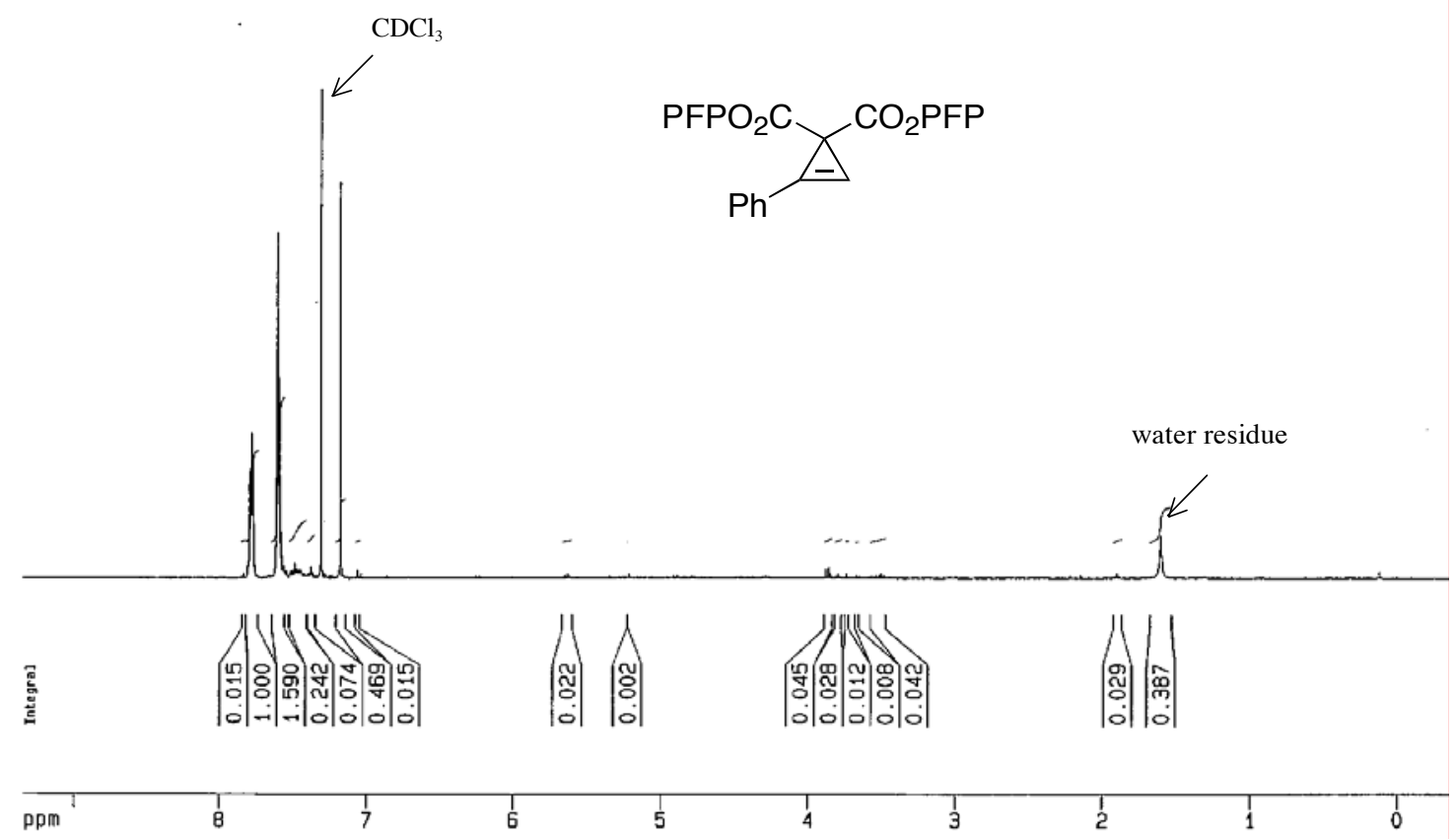

${ }^{13} \mathrm{C}$ NMR spectrum of $\mathbf{4 b}\left(100 \mathrm{MHz}, \mathrm{CDCl}_{3}\right)$

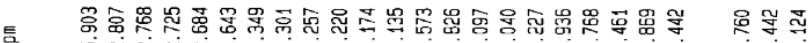

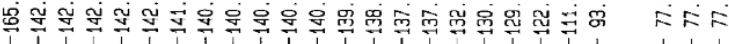

(1)

V

월
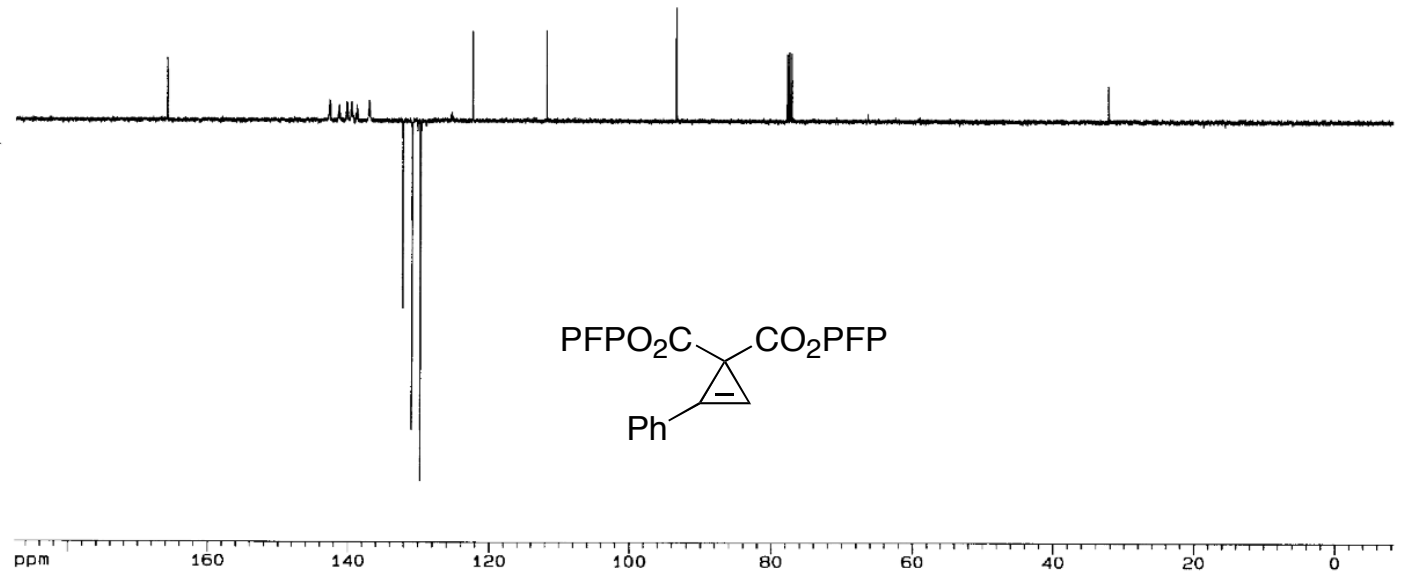


\section{${ }^{1} \mathrm{H}$ NMR spectrum of $\mathbf{4 c}\left(400 \mathrm{MHz}, \mathrm{CDCl}_{3}\right)$}

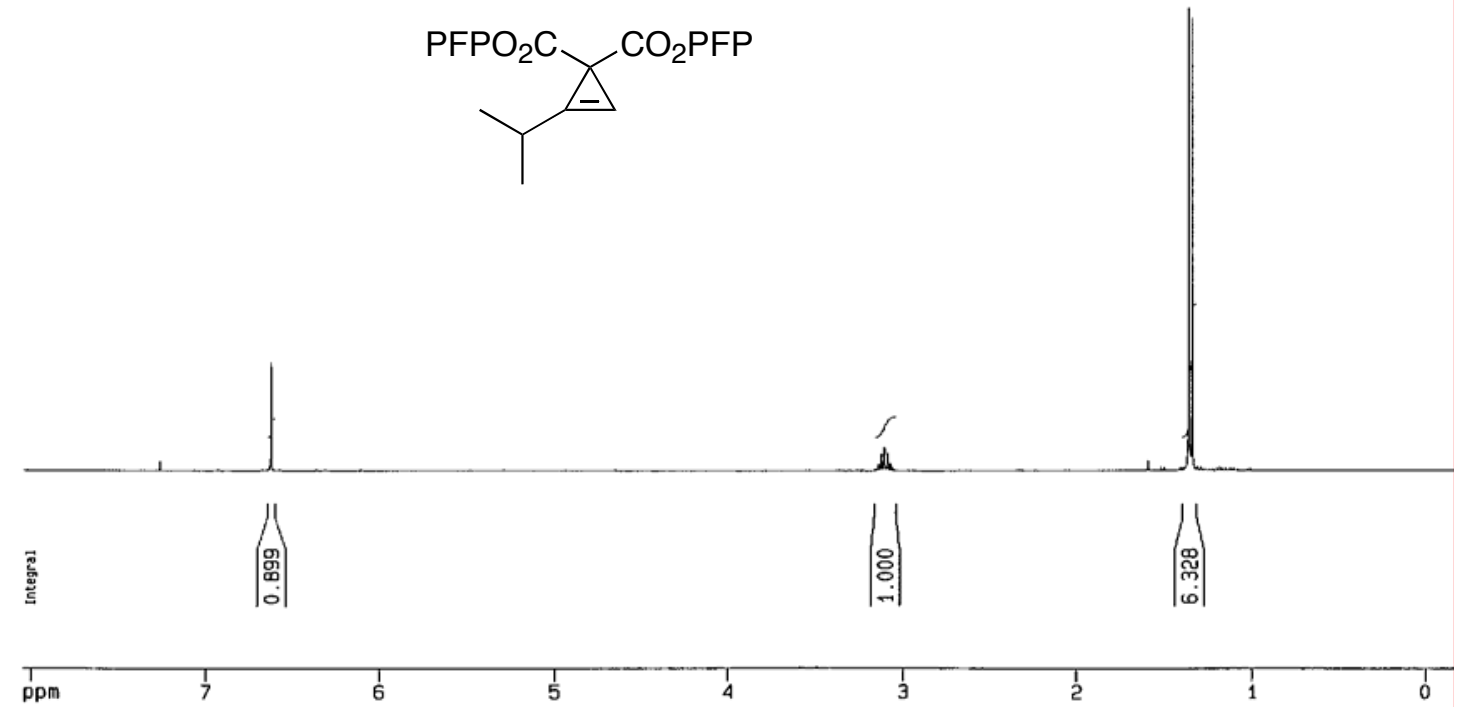

${ }^{13} \mathrm{C}$ NMR spectrum of $4 \mathbf{c}\left(100 \mathrm{MHz}, \mathrm{CDCl}_{3}\right)$

彧

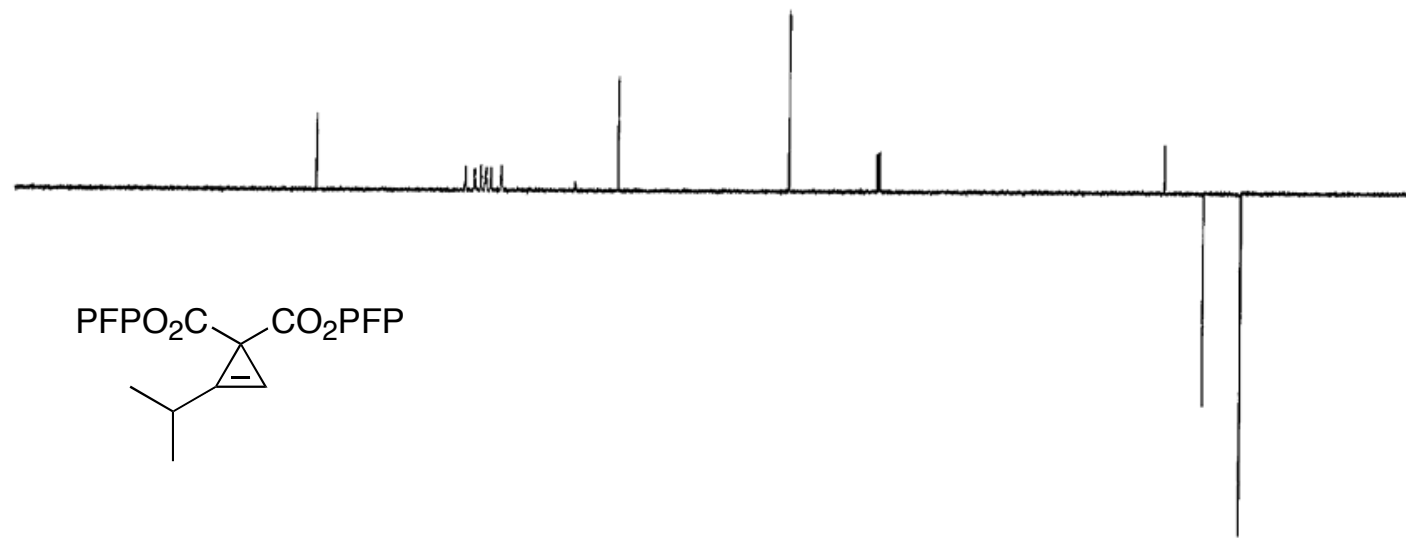


${ }^{1} \mathrm{H}$ NMR spectrum of $\mathbf{4 d}\left(400 \mathrm{MHz}, \mathrm{CDCl}_{3}, \sim 94 \%\right.$ purity)

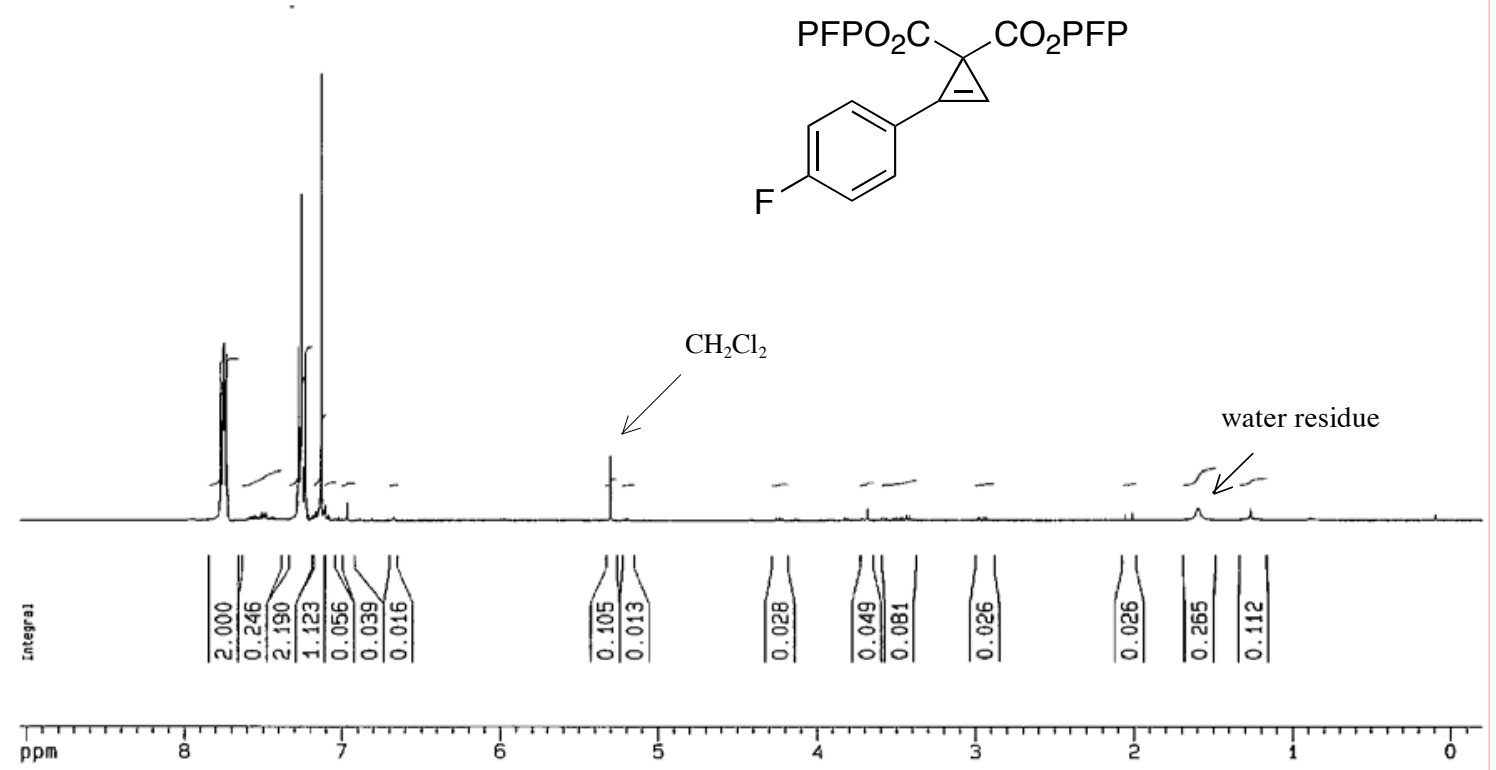

${ }^{15} \mathrm{C}$ NMR spectrum of $\mathbf{4 d}\left(100 \mathrm{MHz}, \mathrm{CDCl}_{3}\right)$

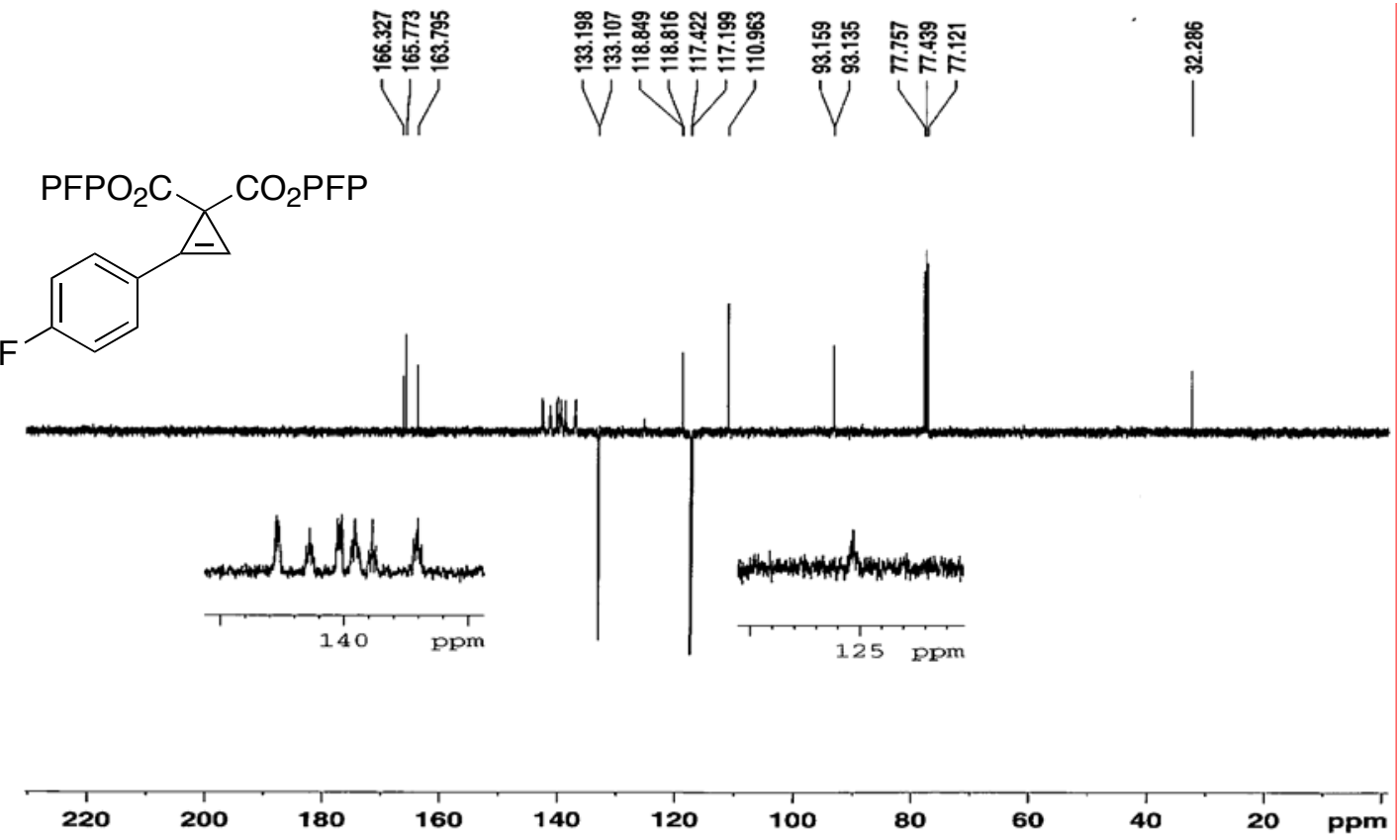


${ }^{1} \mathrm{H}$ NMR spectrum of $\mathbf{4 e}\left(400 \mathrm{MHz}, \mathrm{CDCl}_{3}\right)$

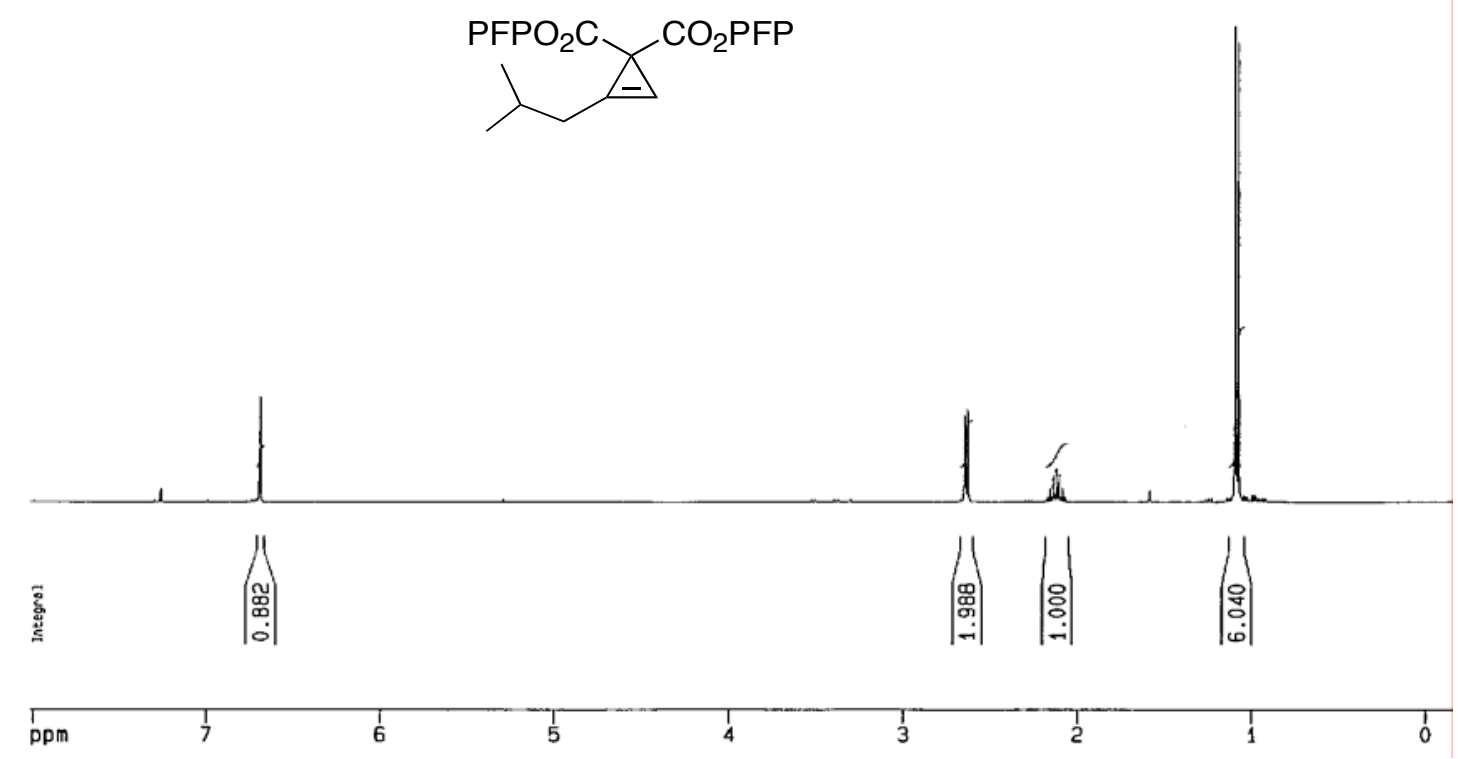

${ }^{13} \mathrm{C}$ NMR spectrum of $\mathbf{4 e}\left(100 \mathrm{MHz}, \mathrm{CDCl}_{3}\right)$

高
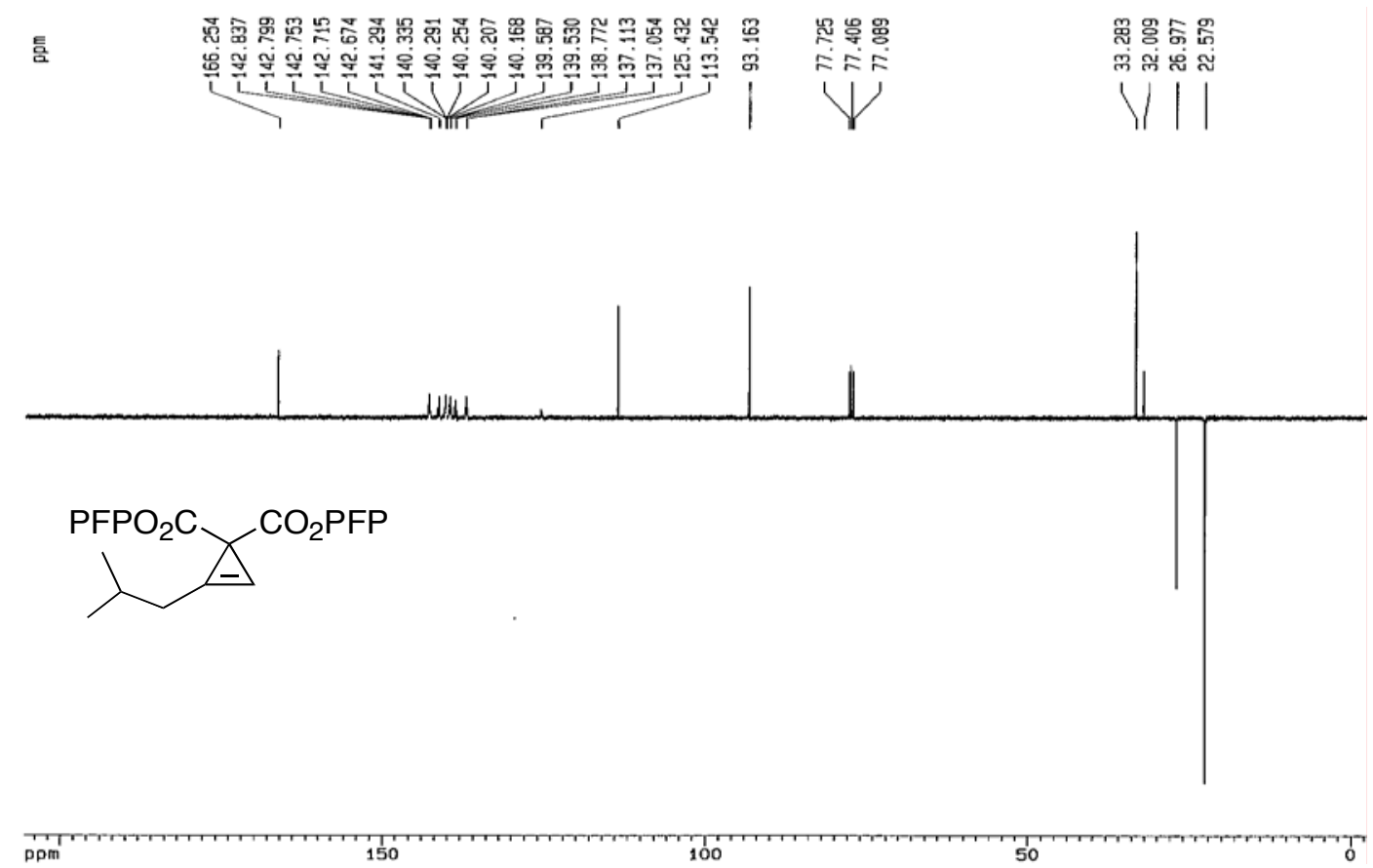
${ }^{1} \mathrm{H}$ NMR spectrum of $\mathbf{4 f}\left(400 \mathrm{MHz}, \mathrm{CDCl}_{3}\right)$

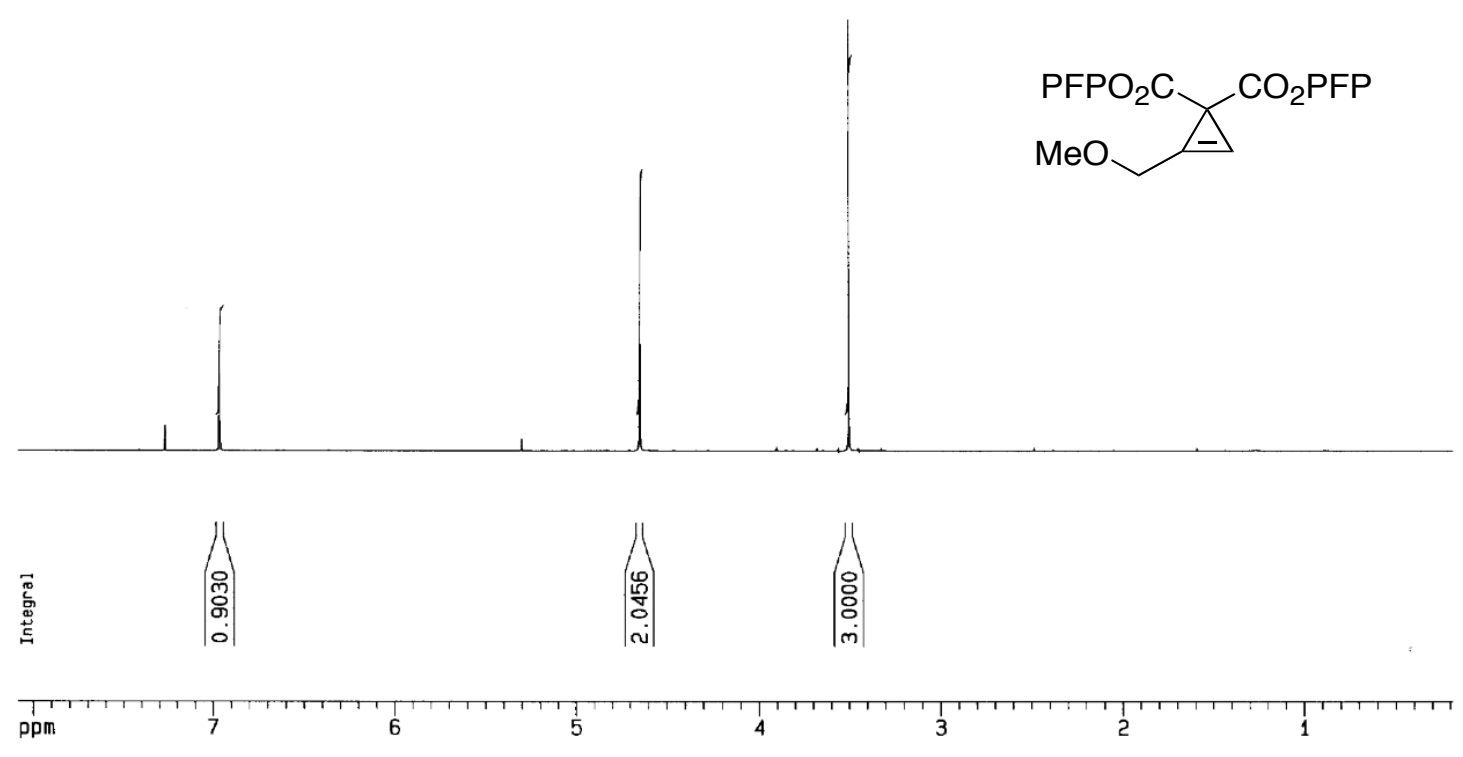

${ }^{13} \mathrm{C}$ NMR spectrum of $\mathbf{4 f}\left(100 \mathrm{MHz}, \mathrm{CDCl}_{3}\right)$
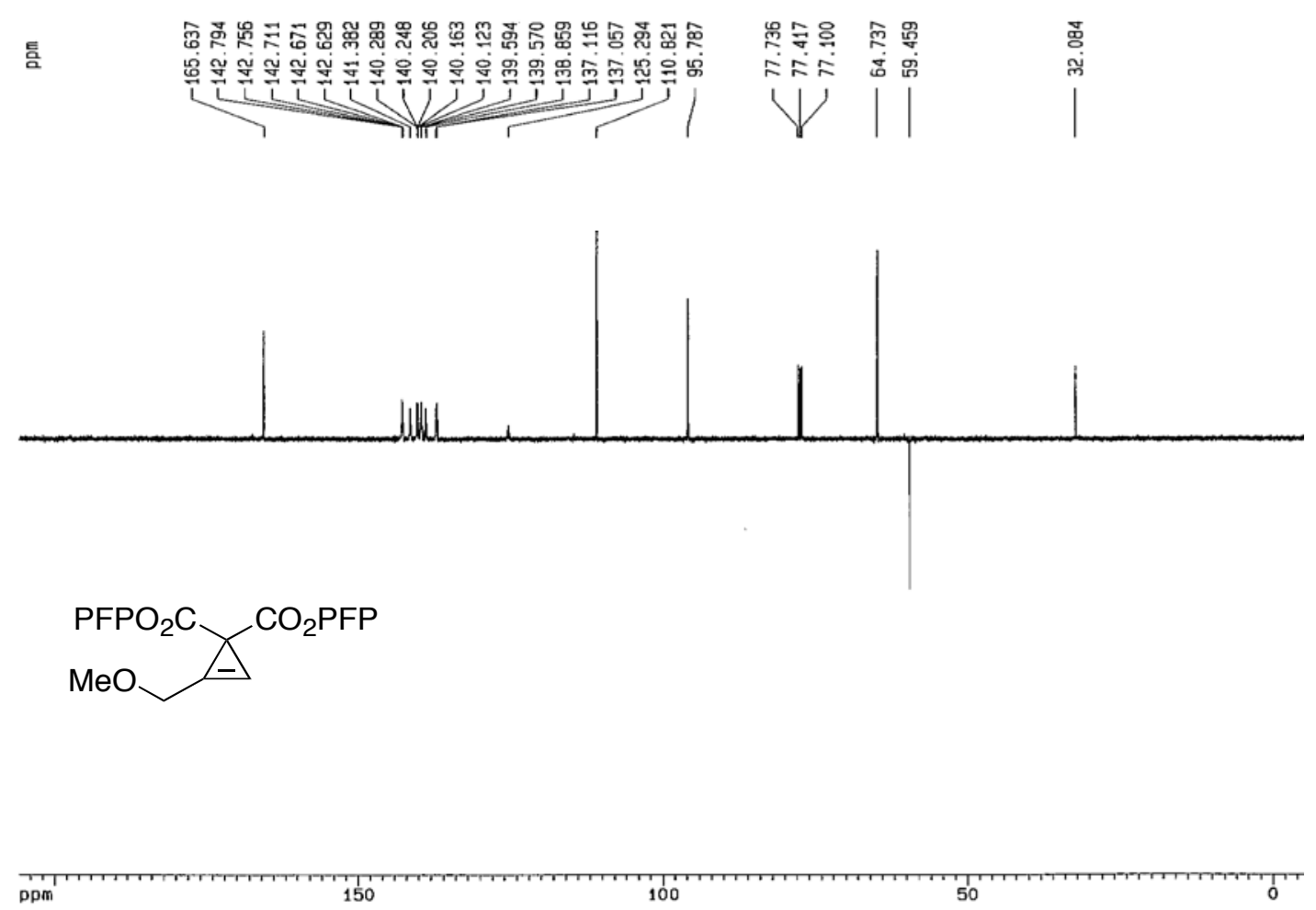
${ }^{1} \mathrm{H}$ NMR spectrum of $\mathbf{4 g}\left(400 \mathrm{MHz}, \mathrm{CDCl}_{3}, \sim 95 \%\right.$ purity)

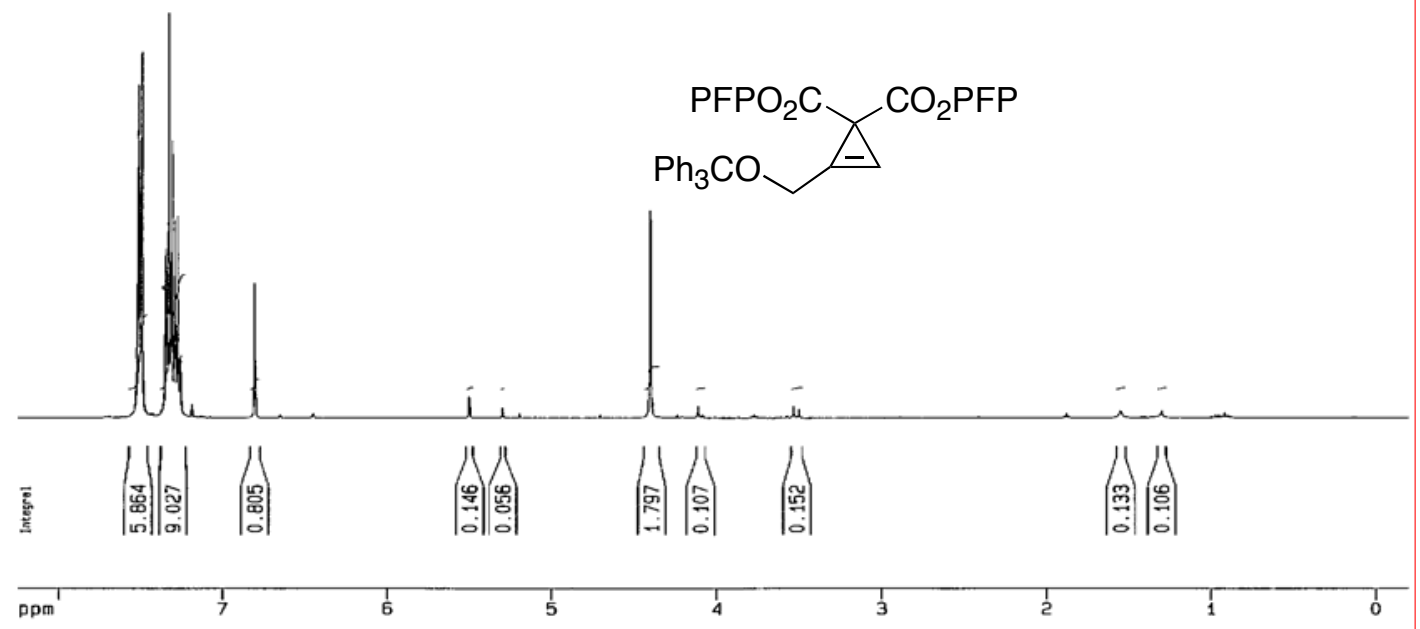

${ }^{13} \mathrm{C}$ NMR spectrum of $\mathbf{4 g}\left(100 \mathrm{MHz}, \mathrm{CDCl}_{3}\right)$

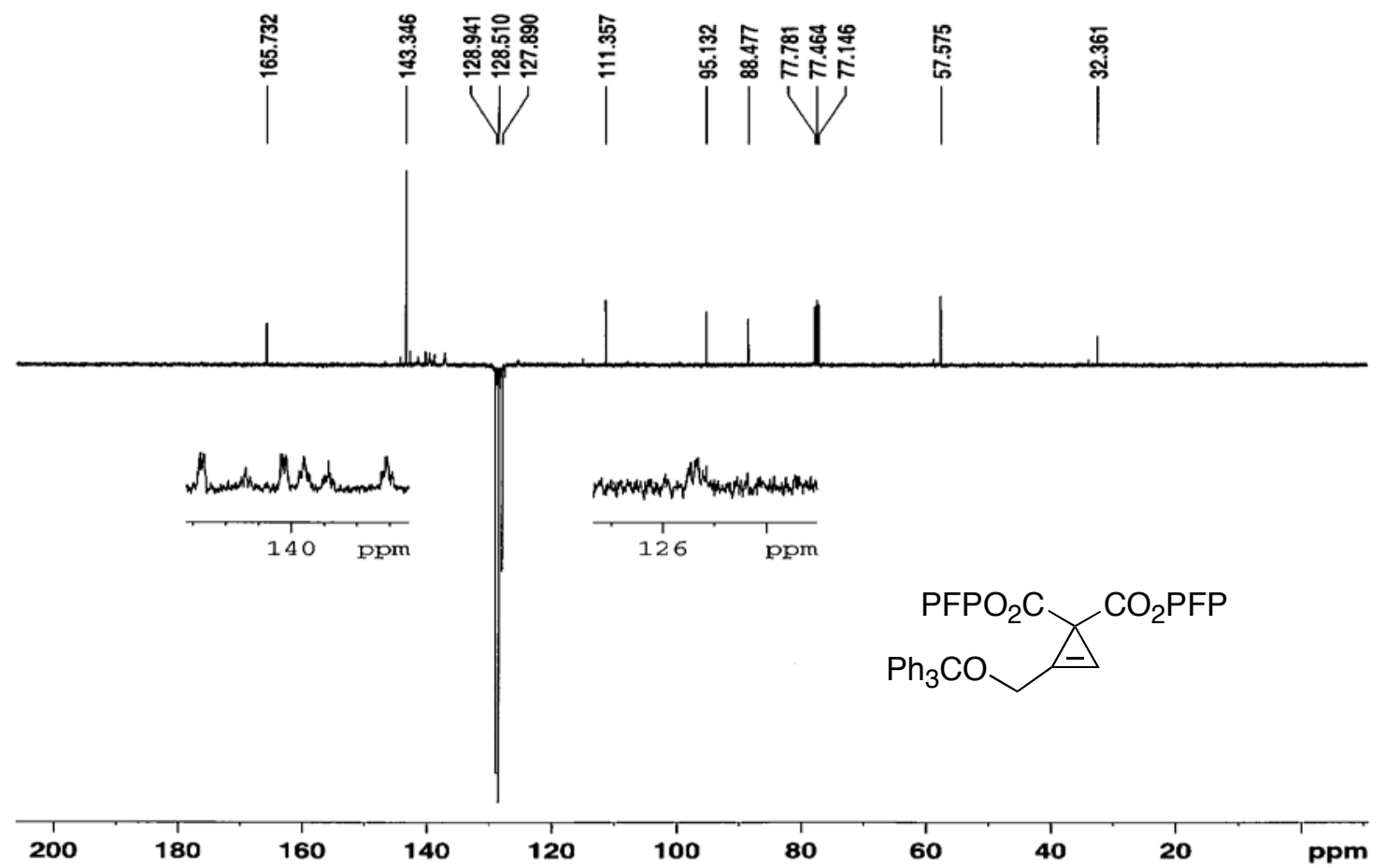


${ }^{1} \mathrm{H}$ NMR spectrum of $\mathbf{5 a}$ (major) (400 $\mathbf{M H z}, \mathrm{CDCl}_{3}$ )

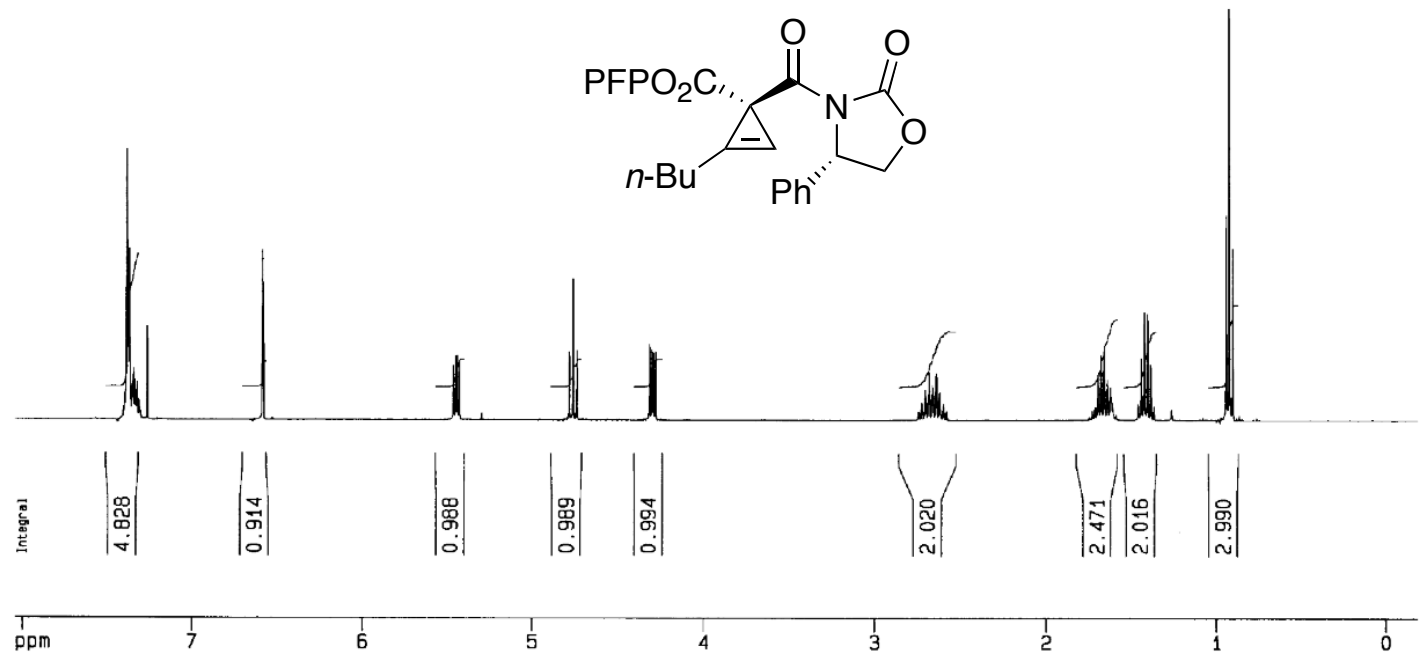

${ }^{15} \mathrm{C}$ NMR spectrum of $\mathbf{5 a}$ (major) (100 $\mathrm{MHz}, \mathrm{CDCl}_{3}$ )

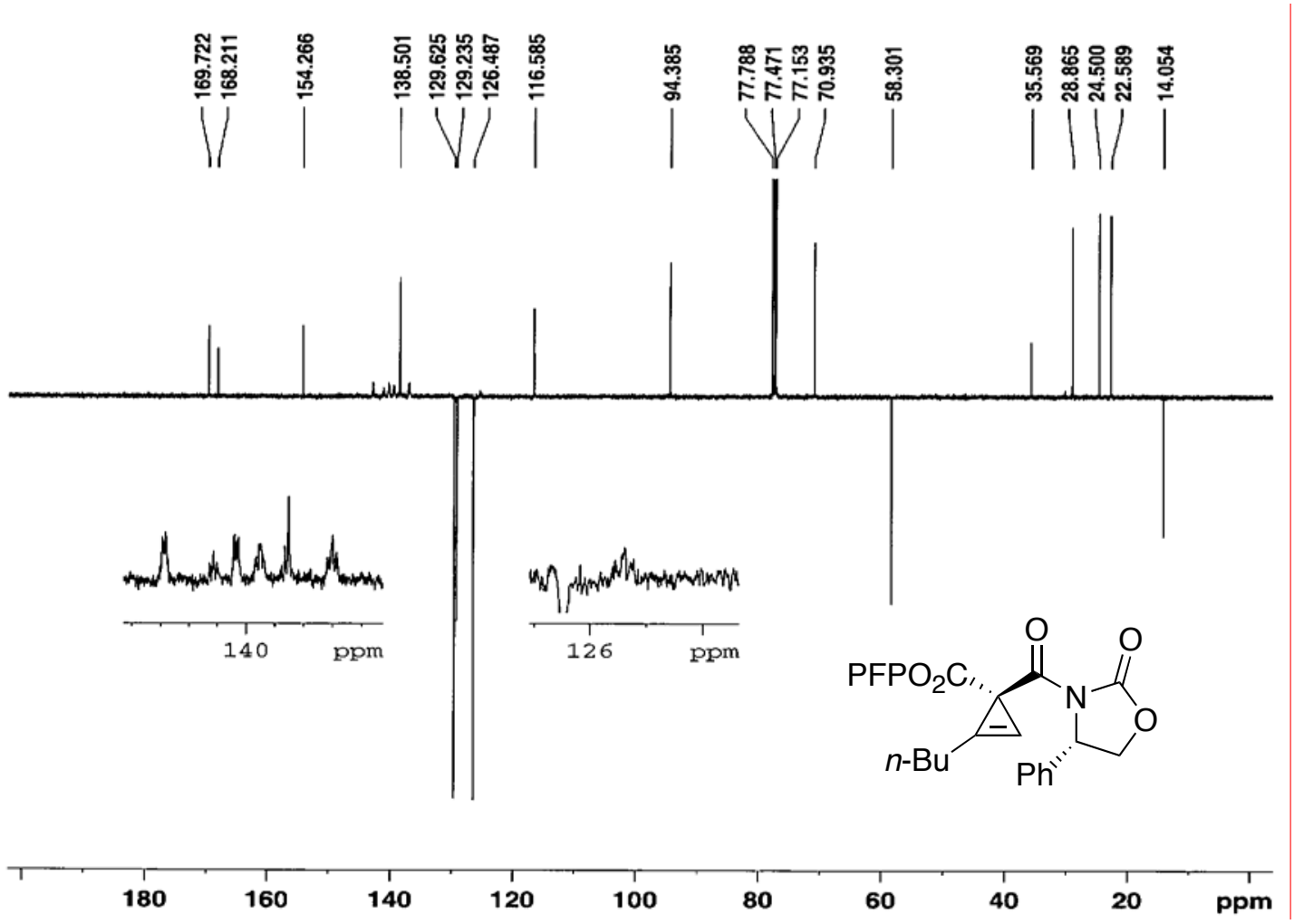


${ }^{1} \mathrm{H}$ NMR spectrum of $\mathbf{5 a}$ (minor) (400 $\left.\mathbf{M H z}, \mathrm{CDCl}_{3}\right)$

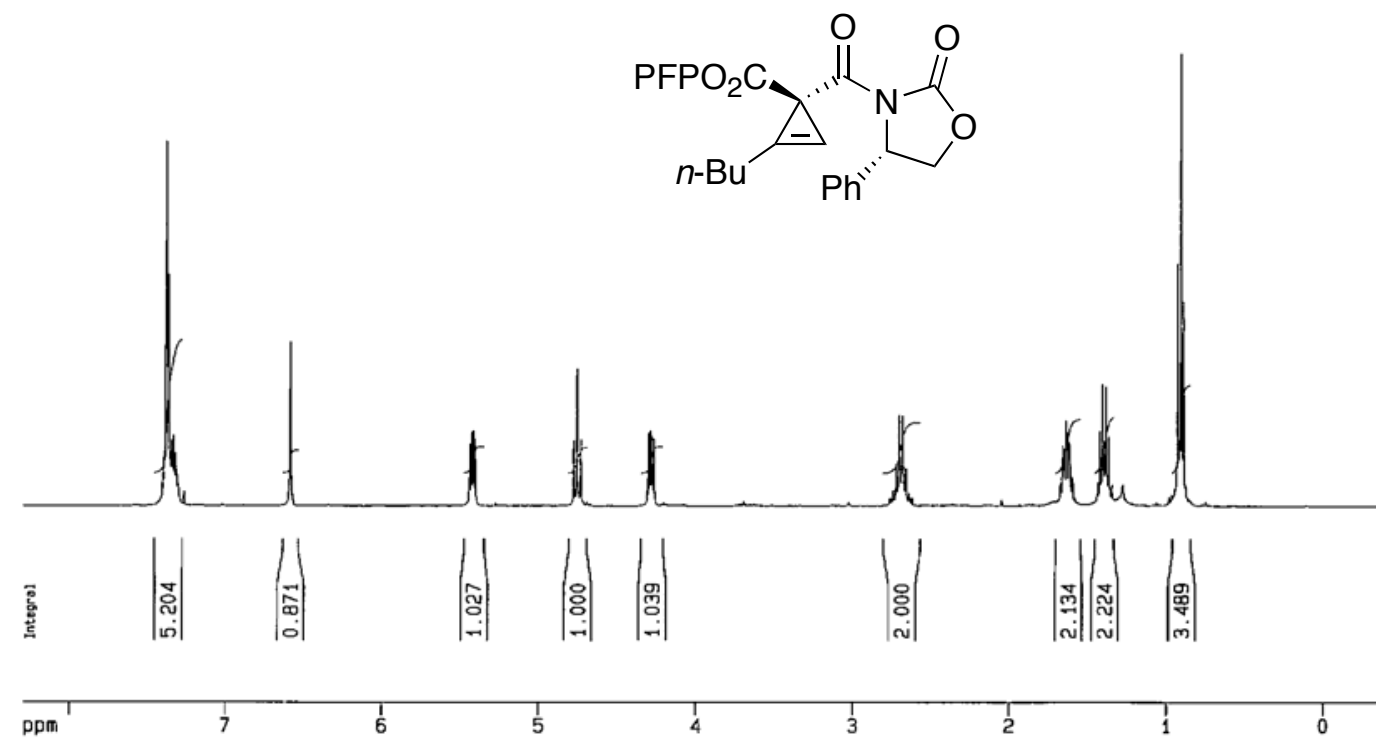

${ }^{13} \mathrm{C}$ NMR spectrum of $\mathbf{5 a}$ (minor) (100 $\mathrm{MHz}, \mathrm{CDCl}_{3}$ )

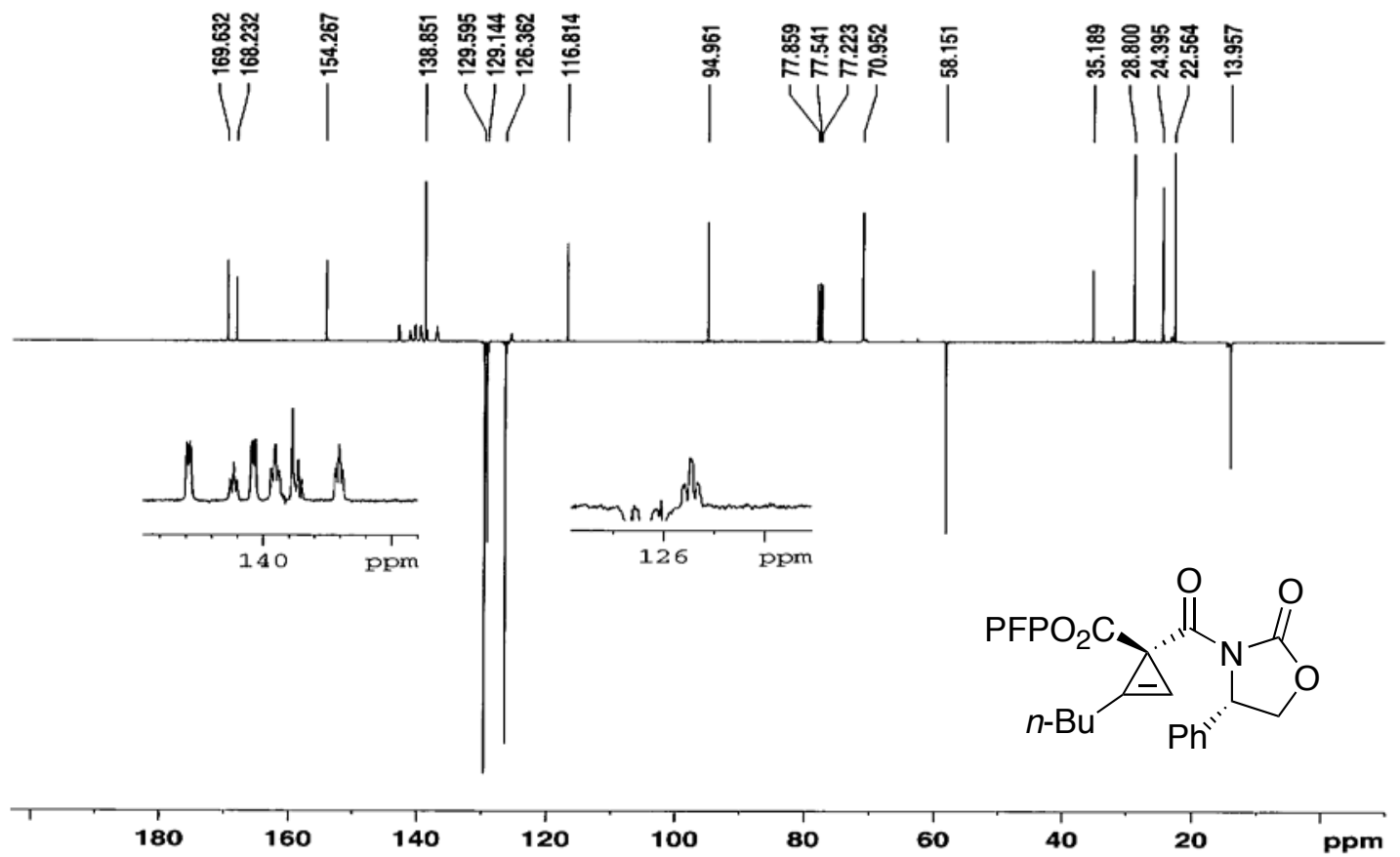


${ }^{1} \mathrm{H}$ NMR spectrum of $\mathbf{5 b}(\boldsymbol{R} \mathbf{c y}, \boldsymbol{S o x})\left(400 \mathrm{MHz}, \mathrm{CDCl}_{3}\right)$

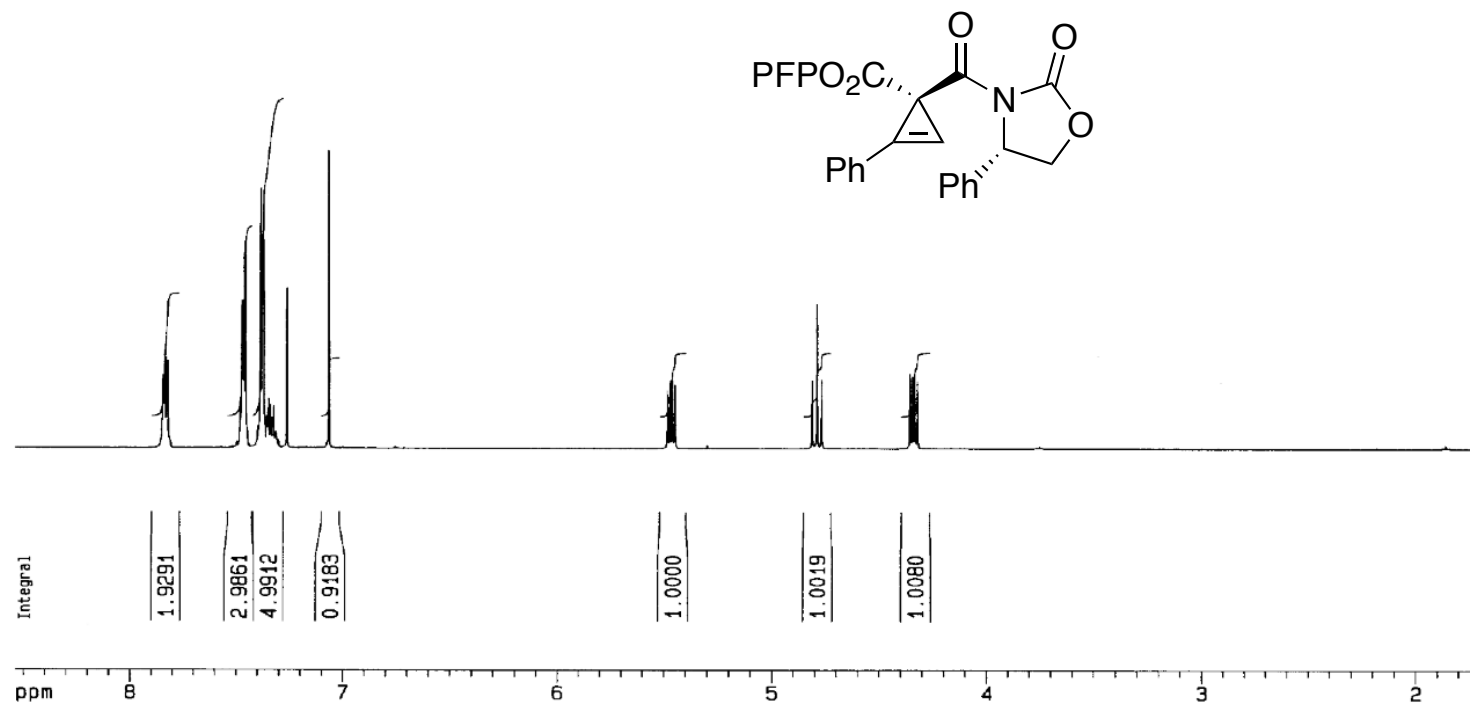

${ }^{13} \mathrm{C}$ NMR spectrum of $\mathbf{5 b}(\boldsymbol{R} \mathbf{c y}, \boldsymbol{S o x})\left(100 \mathrm{MHz}, \mathrm{CDCl}_{3}\right)$

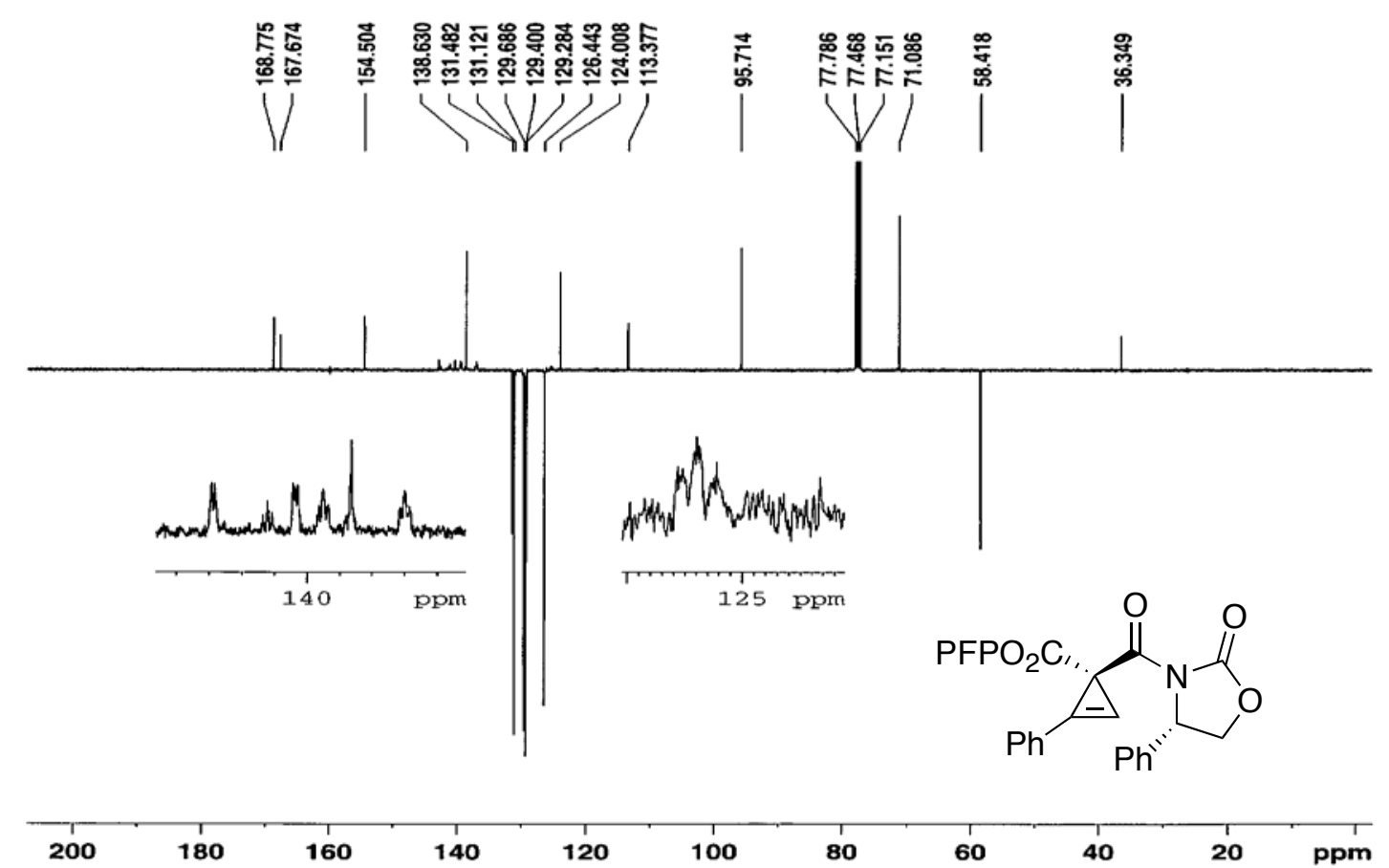


${ }^{1} \mathrm{H}$ NMR spectrum of $\mathbf{5 b}(\mathbf{S c y}, \mathbf{S o x})\left(400 \mathrm{MHz}, \mathrm{CDCl}_{3}, \sim 70 \%\right.$ purity)

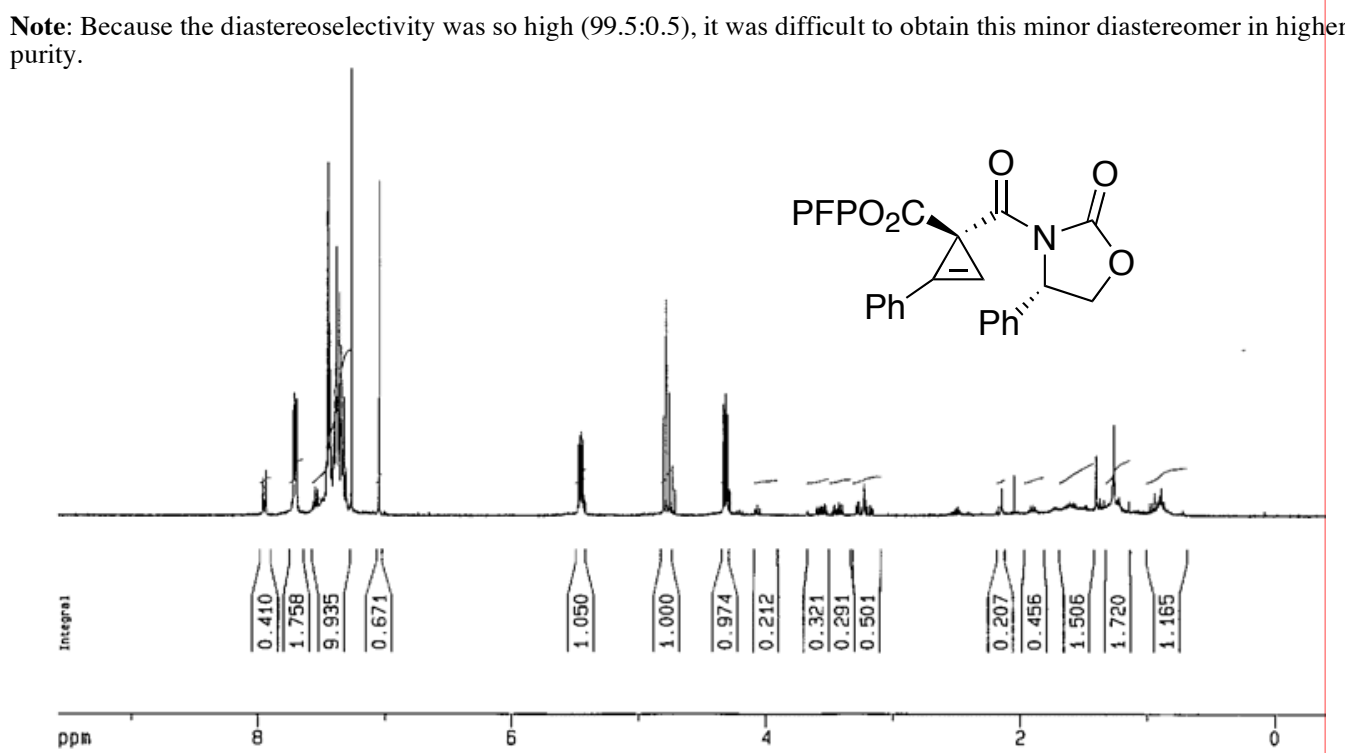

${ }^{19} \mathrm{~F}$ NMR spectrum of $\mathbf{5 b}(\mathbf{S c y}, \mathbf{S o x})\left(376 \mathrm{MHz}^{\mathrm{CDCl}}{ }_{3}\right)$

镸

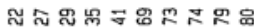

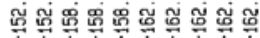

ijijij
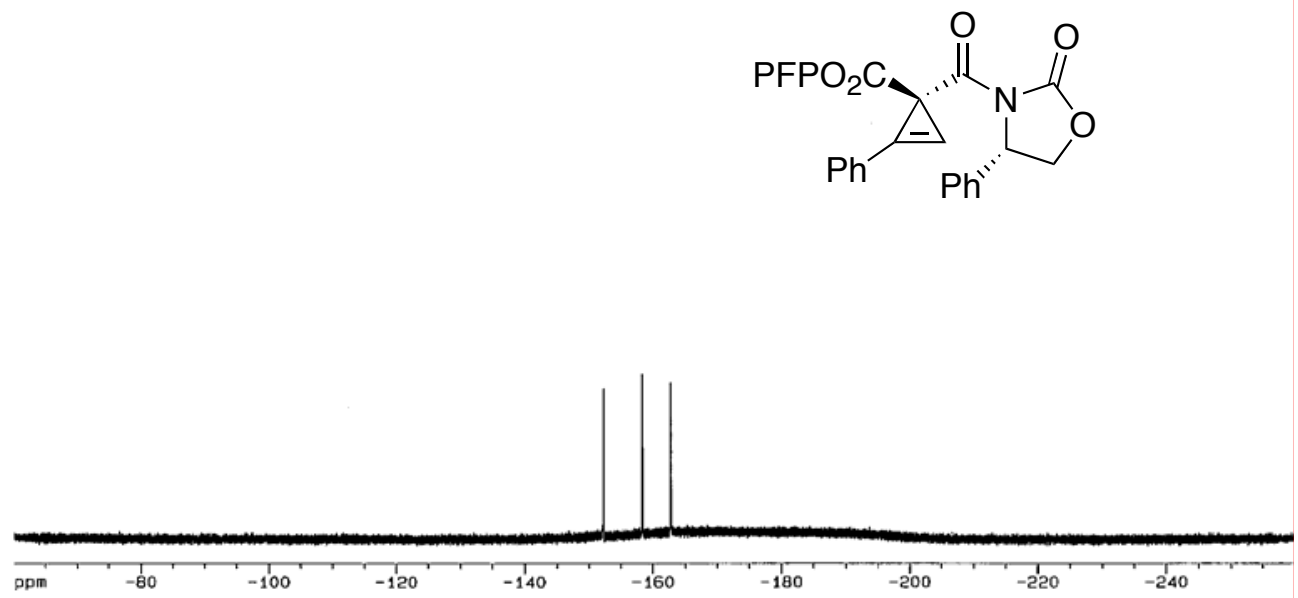
${ }^{1} \mathrm{H}$ NMR spectrum of $\mathbf{5 c}$ (major) (400 $\mathbf{M H z}, \mathrm{CDCl}_{3}$ )
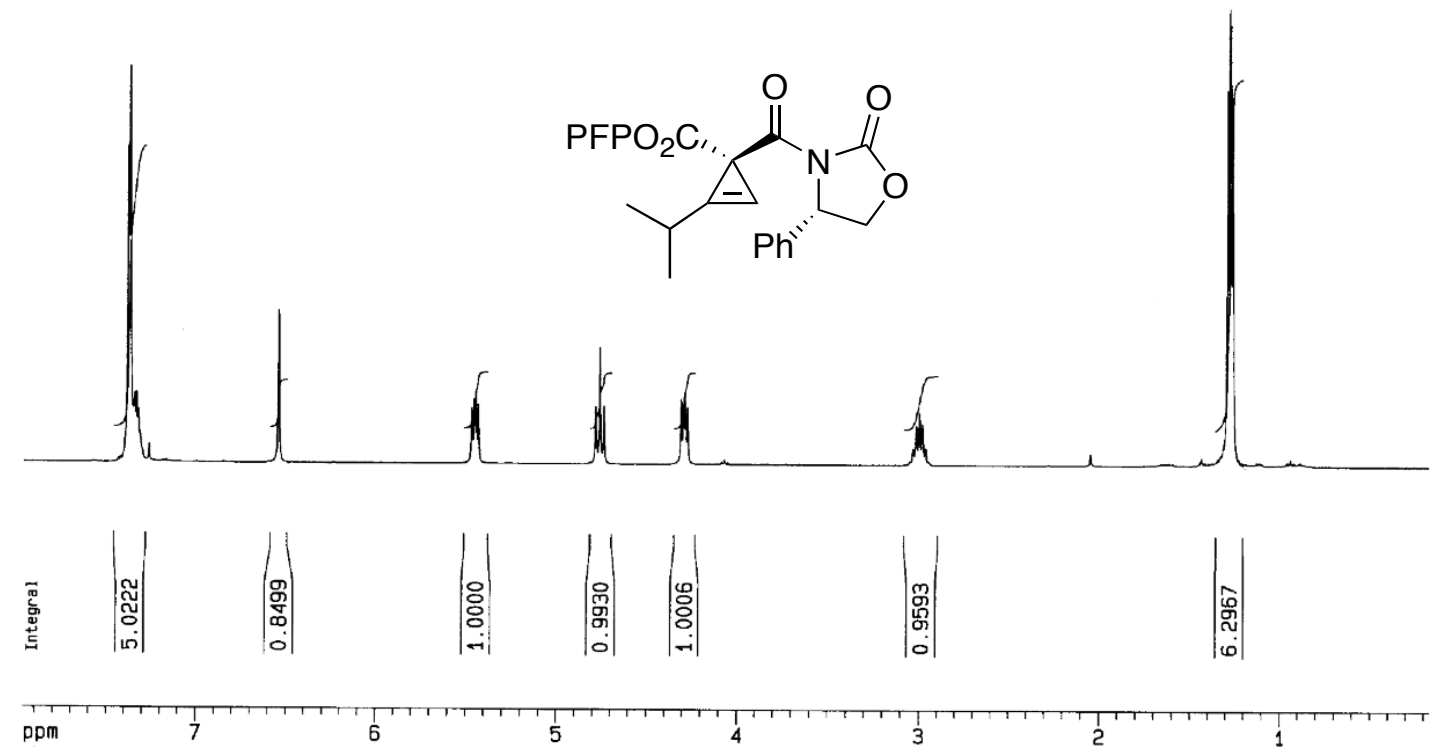

${ }^{13} \mathrm{C}$ NMR spectrum of $\mathbf{5 c}$ (major) $\left(100 \mathrm{MHz}, \mathrm{CDCl}_{3}\right)$

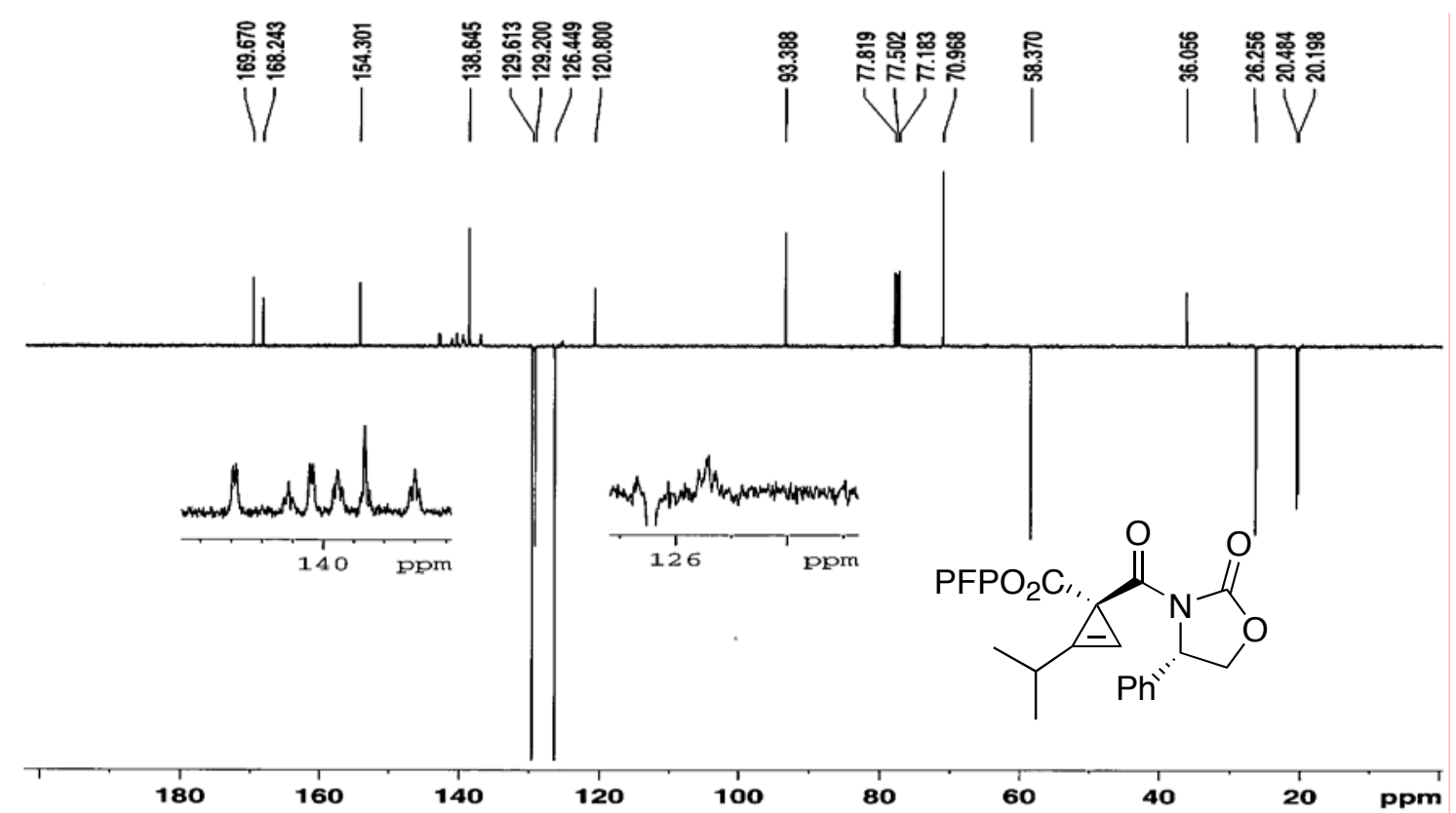


${ }^{1} \mathrm{H}$ NMR spectrum of $\mathbf{5 c}$ (minor) (400 MHz, $\mathrm{CDCl}_{3}$ )

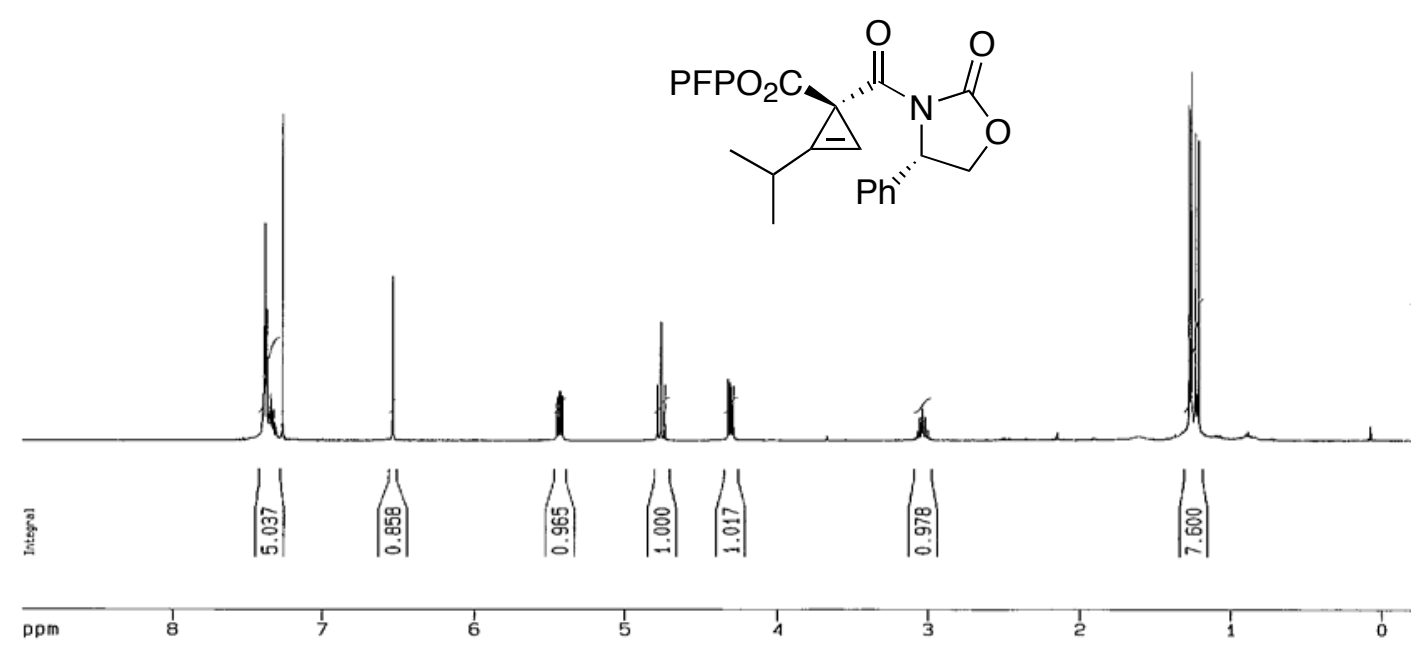

${ }^{13} \mathrm{C}$ NMR spectrum of $\mathbf{5 c}$ (minor) (100 MHz, $\left.\mathrm{CDCl}_{3}\right)$

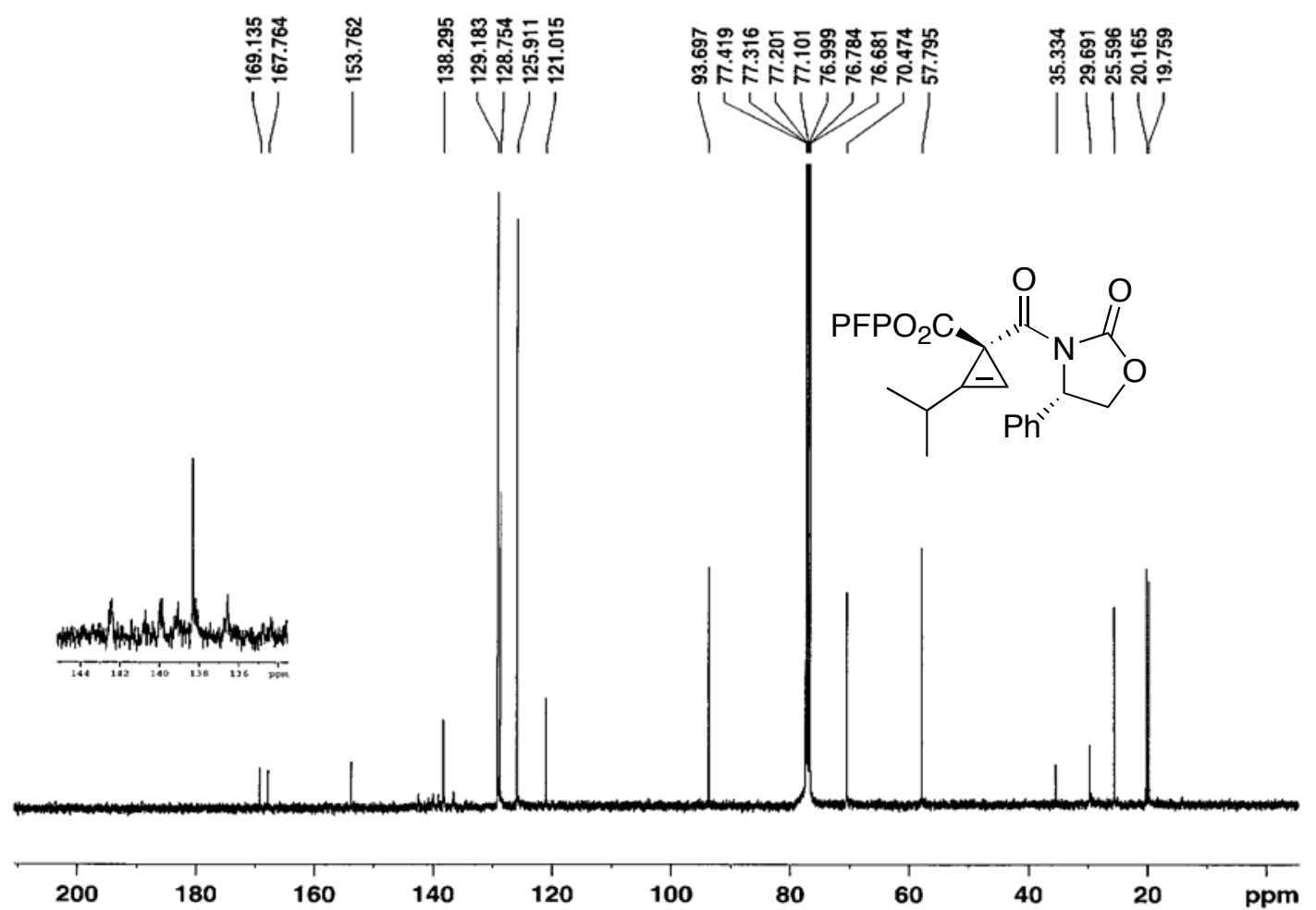


${ }^{1} \mathrm{H}$ NMR spectrum of $\mathbf{5 d}$ (major) (400 $\mathrm{MHz}, \mathrm{CDCl}_{3}$ )

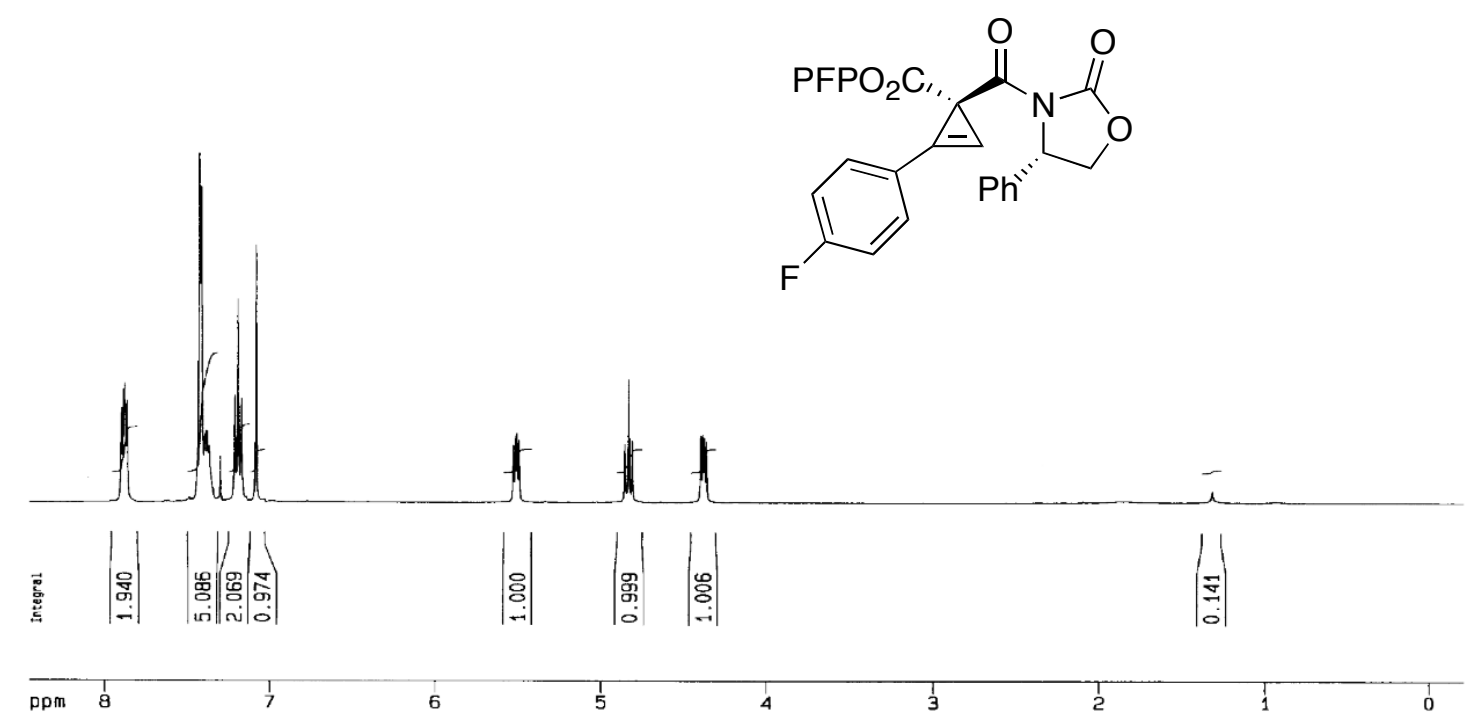

${ }^{13} \mathrm{C}$ NMR spectrum of $\mathbf{5 d}$ (major) (100 $\mathrm{MHz}, \mathrm{CDCl}_{3}$ )

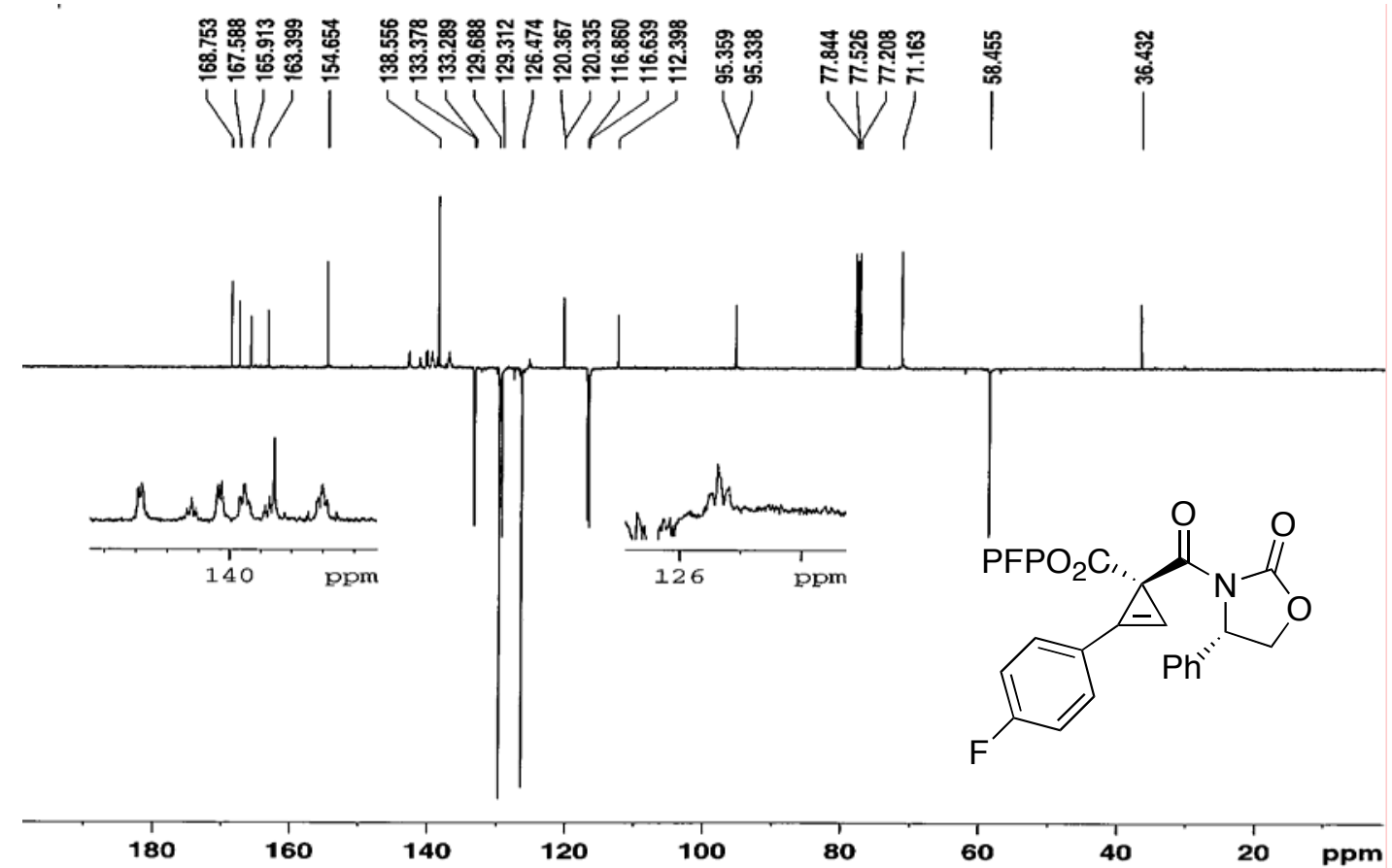


${ }^{1} \mathrm{H}$ NMR spectrum of $\mathbf{5 d}$ (minor) (400 $\mathrm{MHz}, \mathrm{CDCl}_{3}, \sim 70 \%$ purity)

Note: Because the diastereoselectivity was so high (99:1), it was difficult to obtain this minor diastereomer in higher purity.

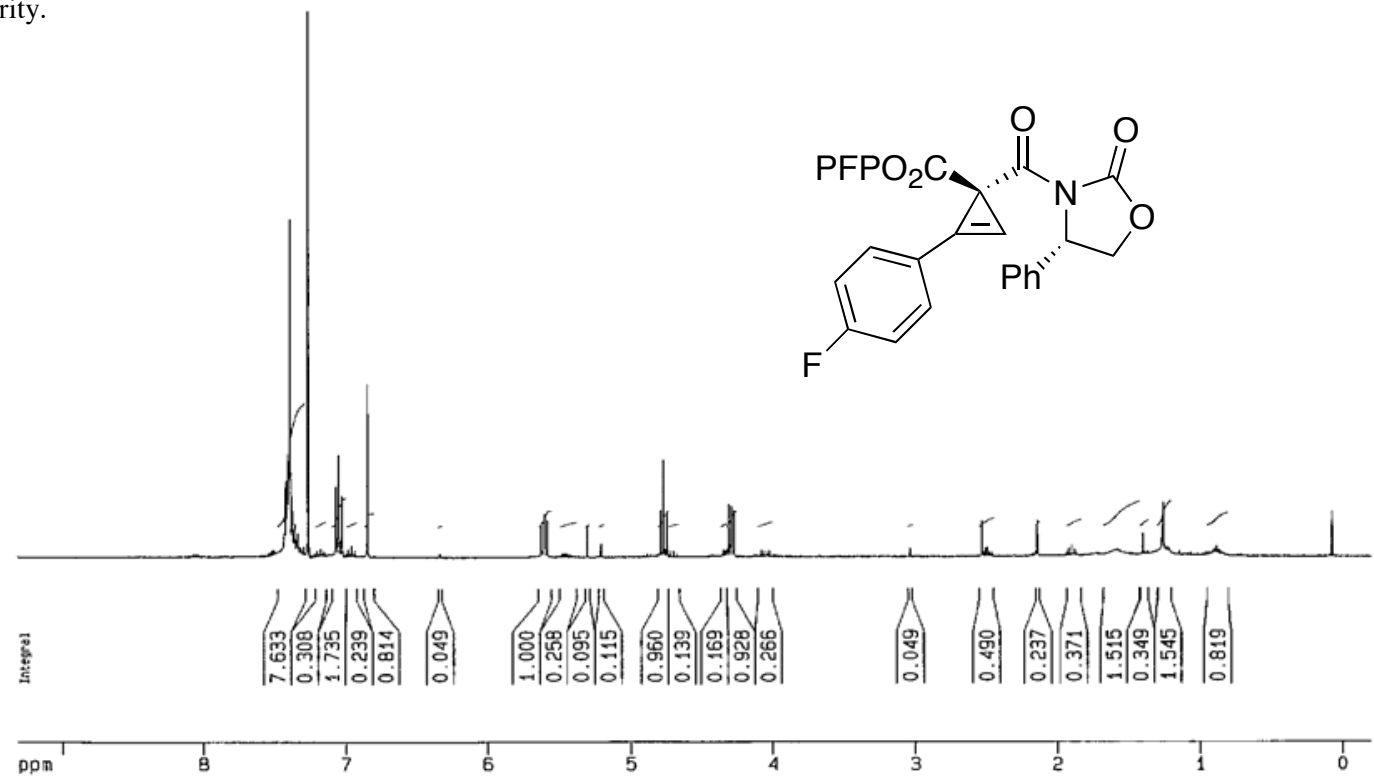

${ }^{19} \mathrm{~F}$ NMR spectrum of $\mathbf{5 d}$ (minor) $\left(376 \mathrm{MHz}, \mathrm{CDCl}_{3}\right)$

镸
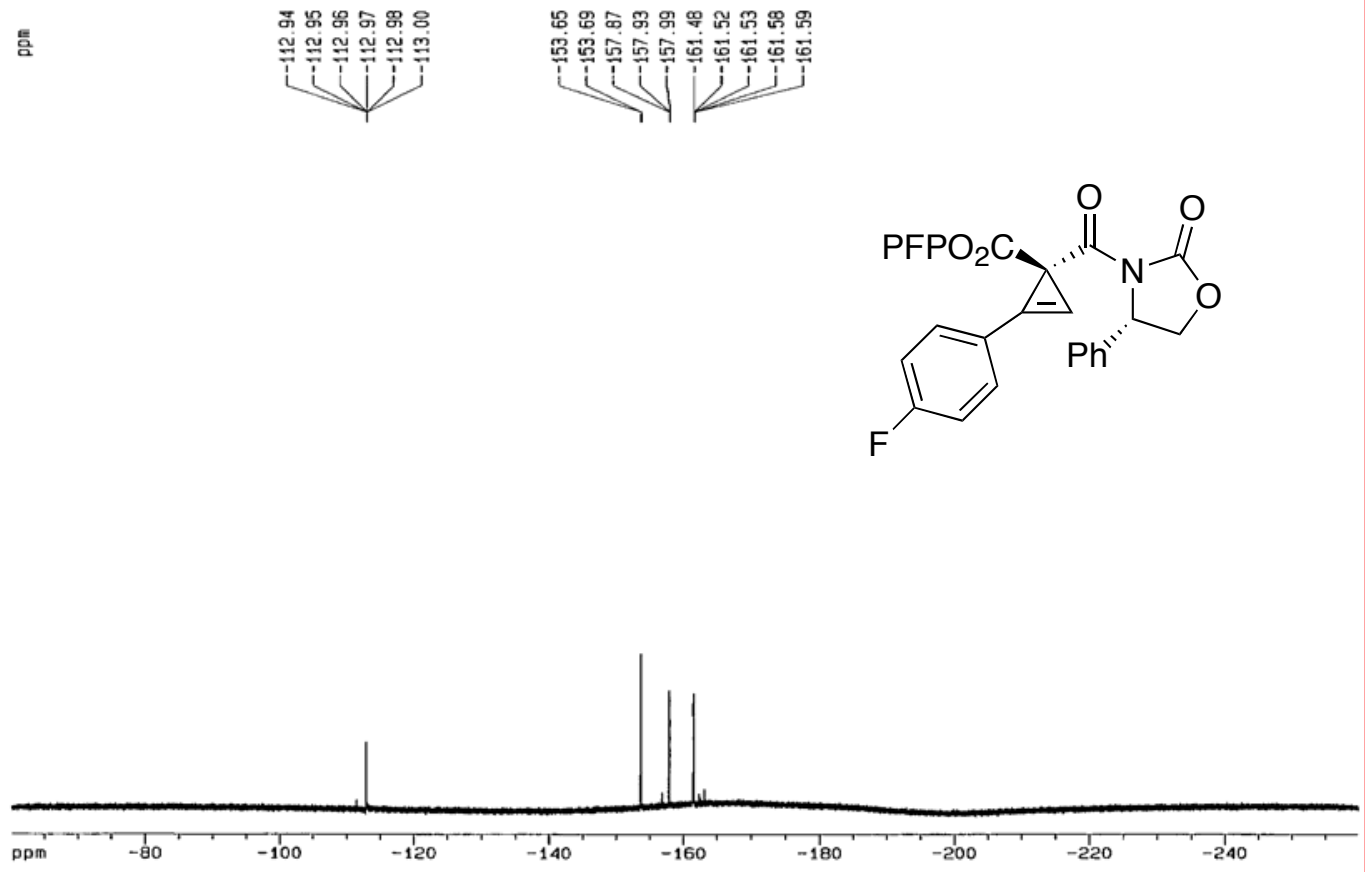
${ }^{1} \mathrm{H}$ NMR spectrum of $\mathbf{5 e}$ (major) (400 $\mathrm{MHz}, \mathrm{CDCl}_{3}$ )

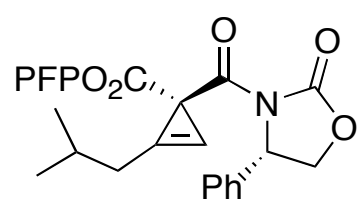
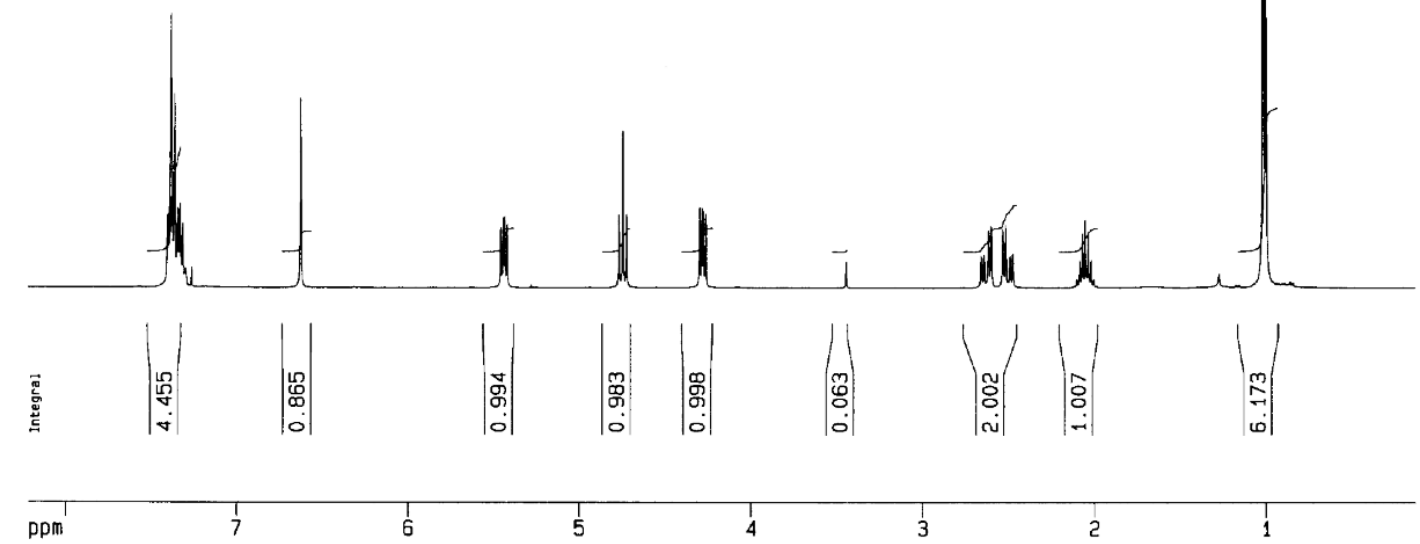

${ }^{13} \mathrm{C}$ NMR spectrum of $\mathbf{5 e}$ (major) (100 $\mathrm{MHz}, \mathrm{CDCl}_{3}$ )

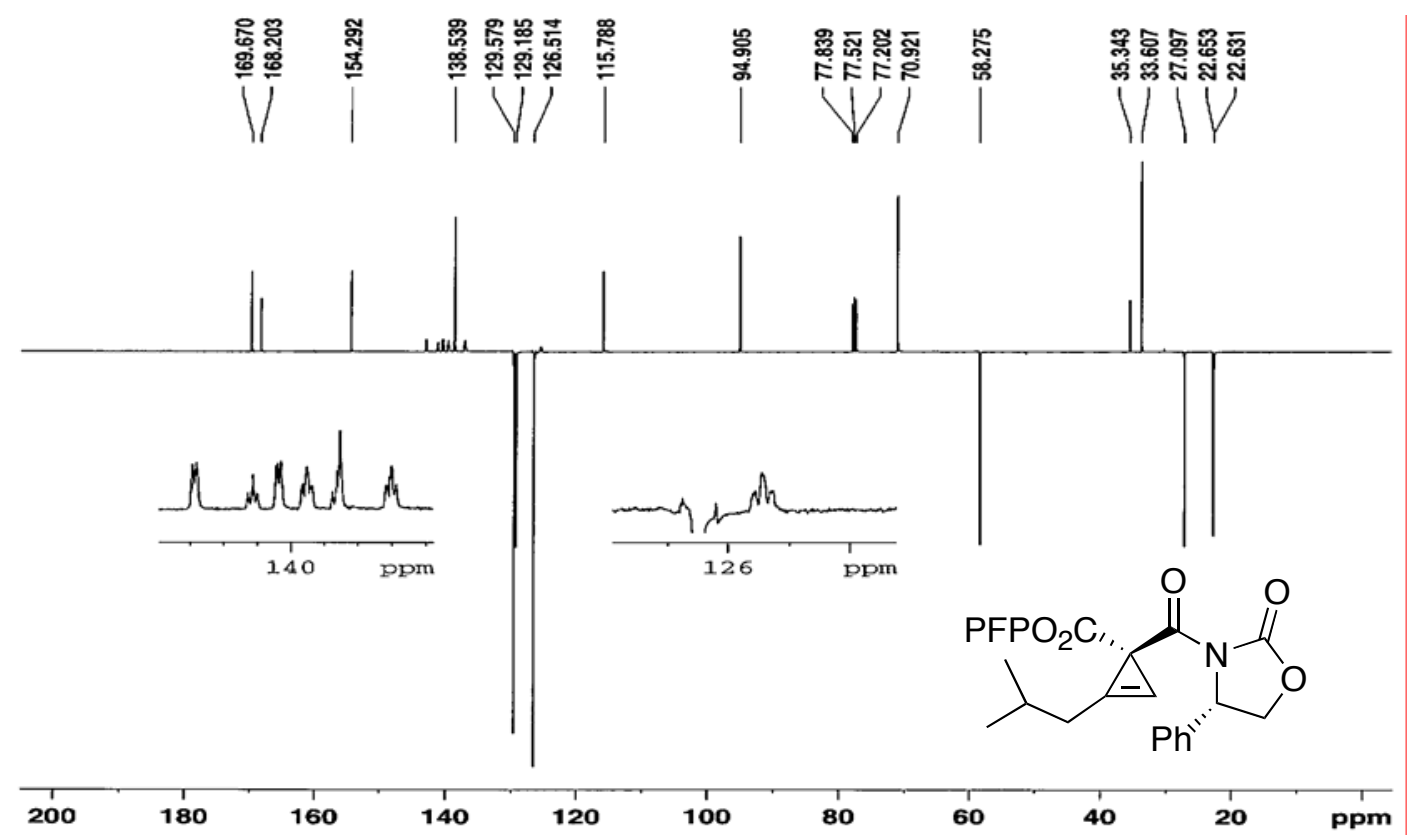


${ }^{1} \mathrm{H}$ NMR spectrum of $\mathbf{5 e}$ (minor) (400 $\mathrm{MHz}, \mathrm{CDCl}_{3}$ )

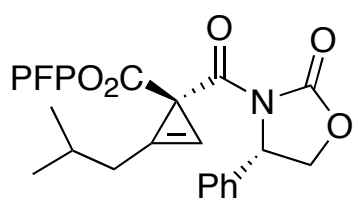

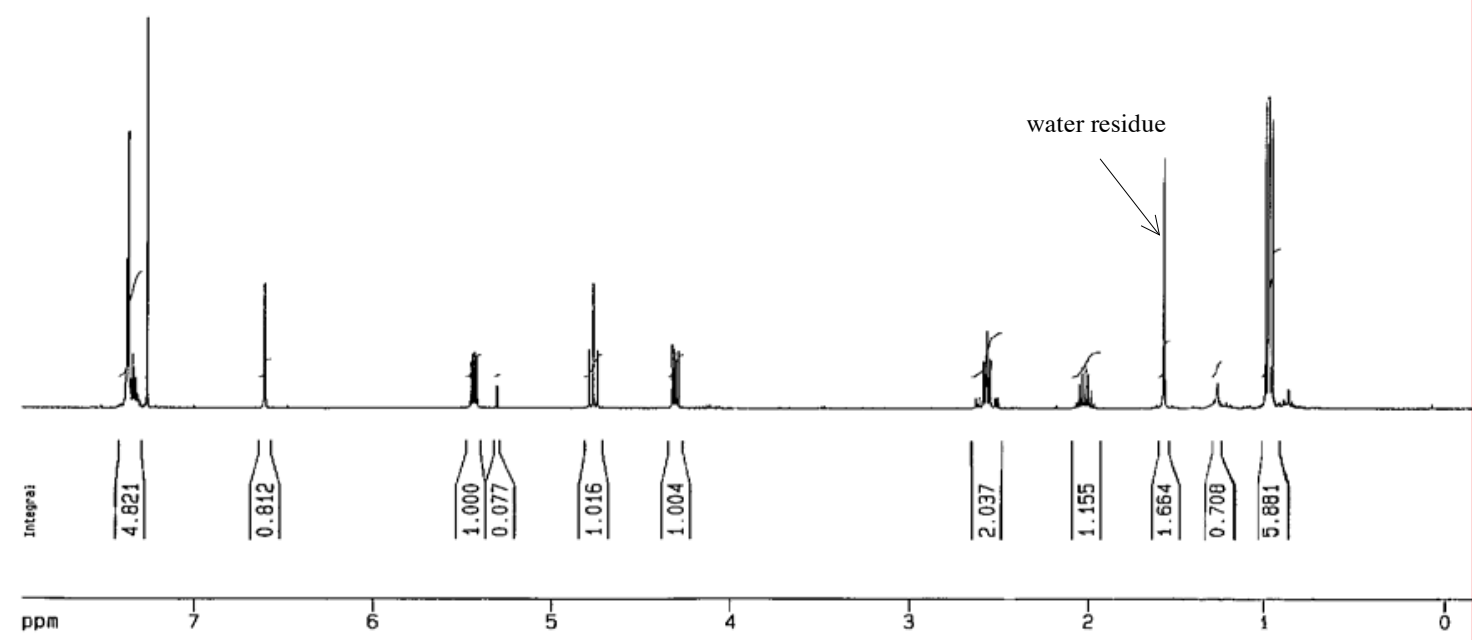

${ }^{13} \mathrm{C}$ NMR spectrum of $\mathbf{5 e}$ (minor) (100 $\mathrm{MHz}, \mathrm{CDCl}_{3}$ )

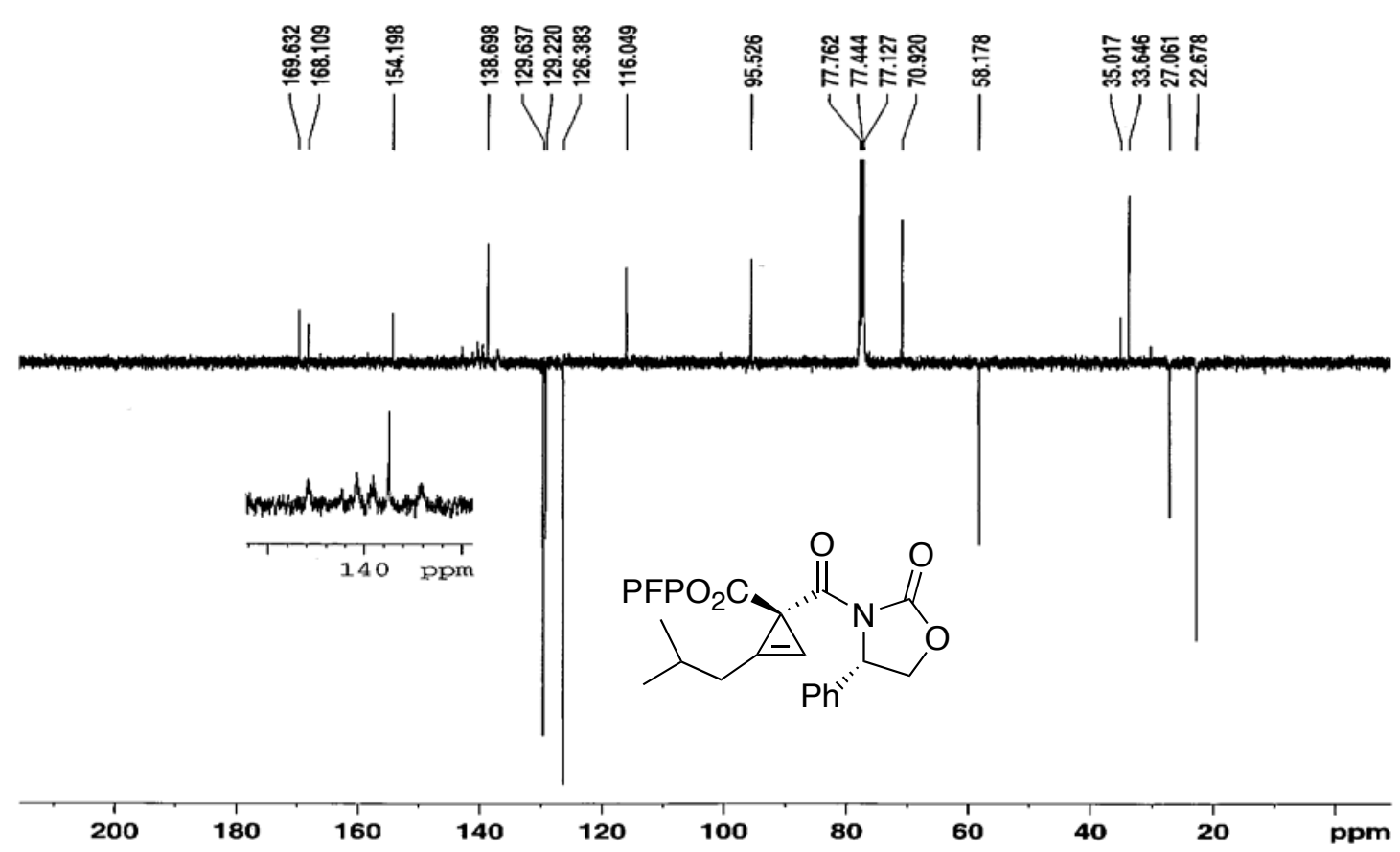


${ }^{1} \mathrm{H}$ NMR spectrum of $\mathbf{5 f}$ (major) (400 $\mathrm{MHz}, \mathrm{CDCl}_{3}$ )
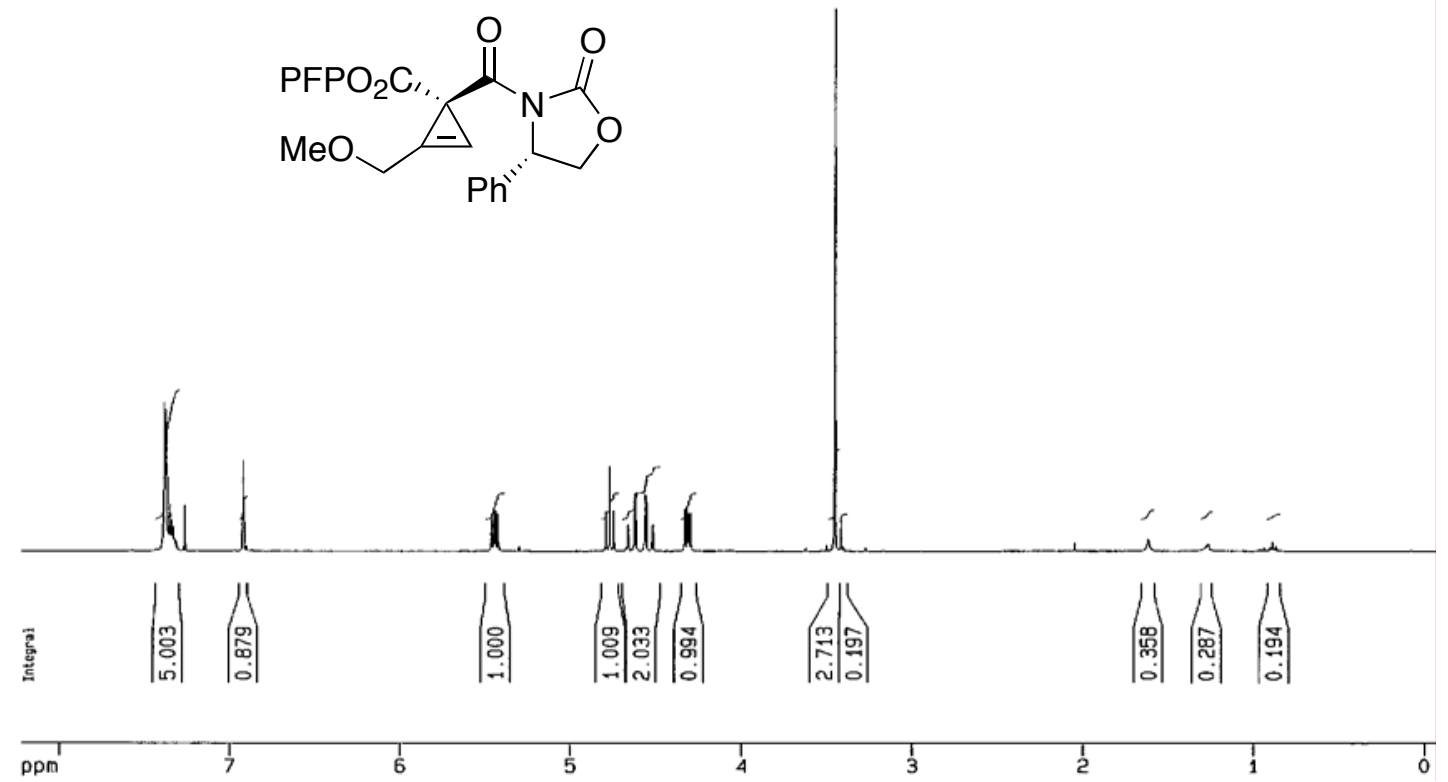

${ }^{13} \mathrm{C}$ NMR spectrum of $\mathbf{5 f}$ (major) $\left(100 \mathrm{MHz}, \mathrm{CDCl}_{3}\right)$

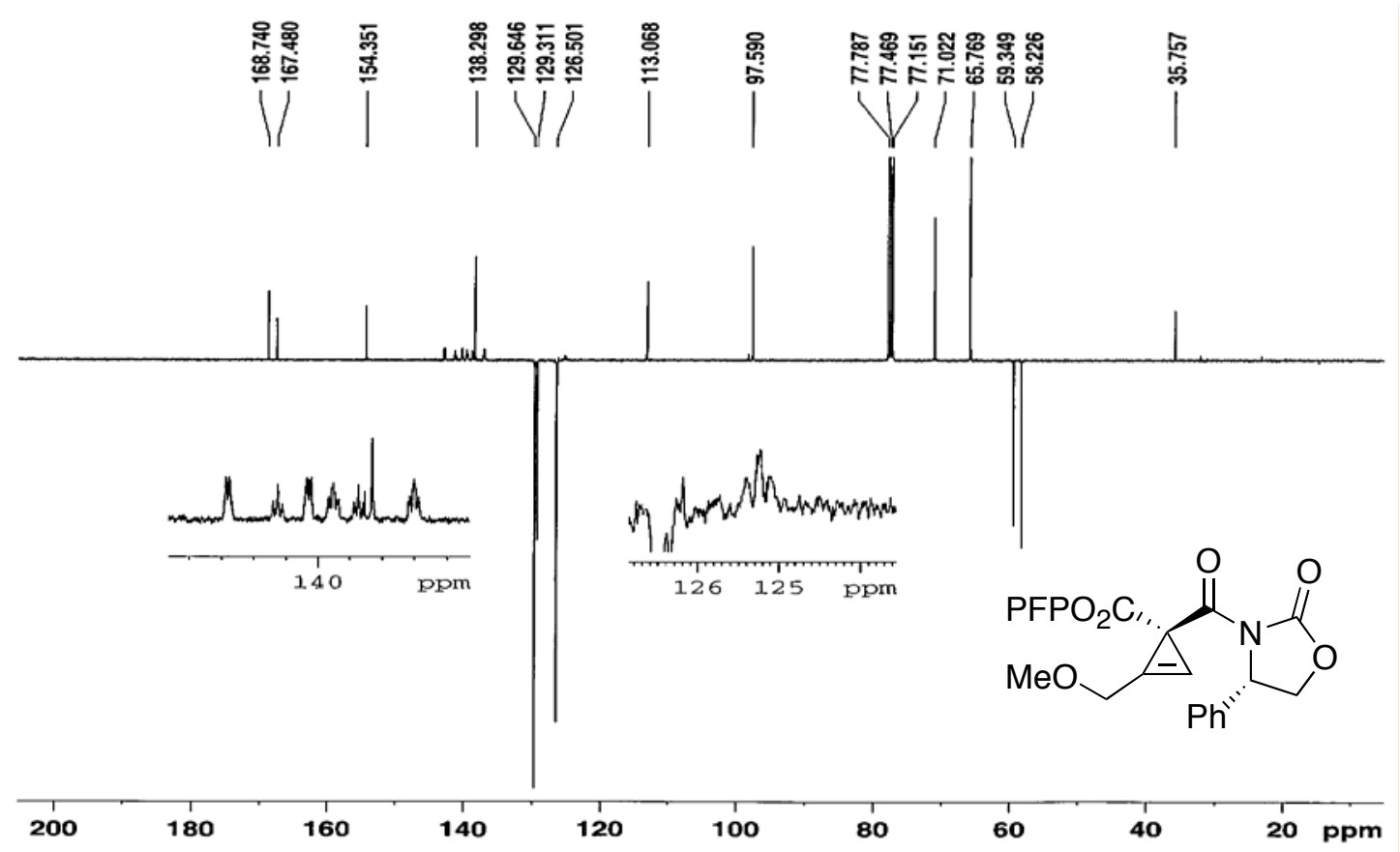


${ }^{1} \mathrm{H}$ NMR spectrum of $\mathbf{5 f}$ (minor) (400 $\mathrm{MHz}, \mathrm{CDCl}_{3}$ )<smiles>COCC1=CC1(C)[C@H]1C[C@@H](c2ccccc2)N1C(=O)C1COC(=O)N1</smiles>

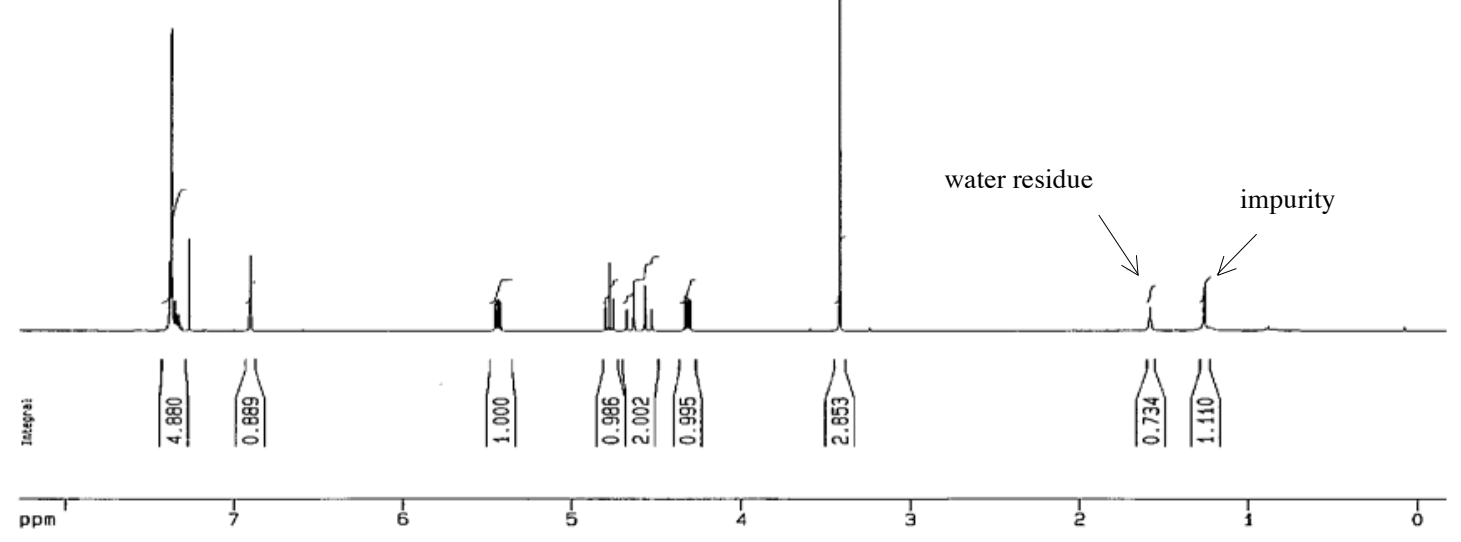

${ }^{13} \mathrm{C}$ NMR spectrum of $\mathbf{5 f}$ (minor) (100 $\mathrm{MHz}, \mathrm{CDCl}_{3}$ )

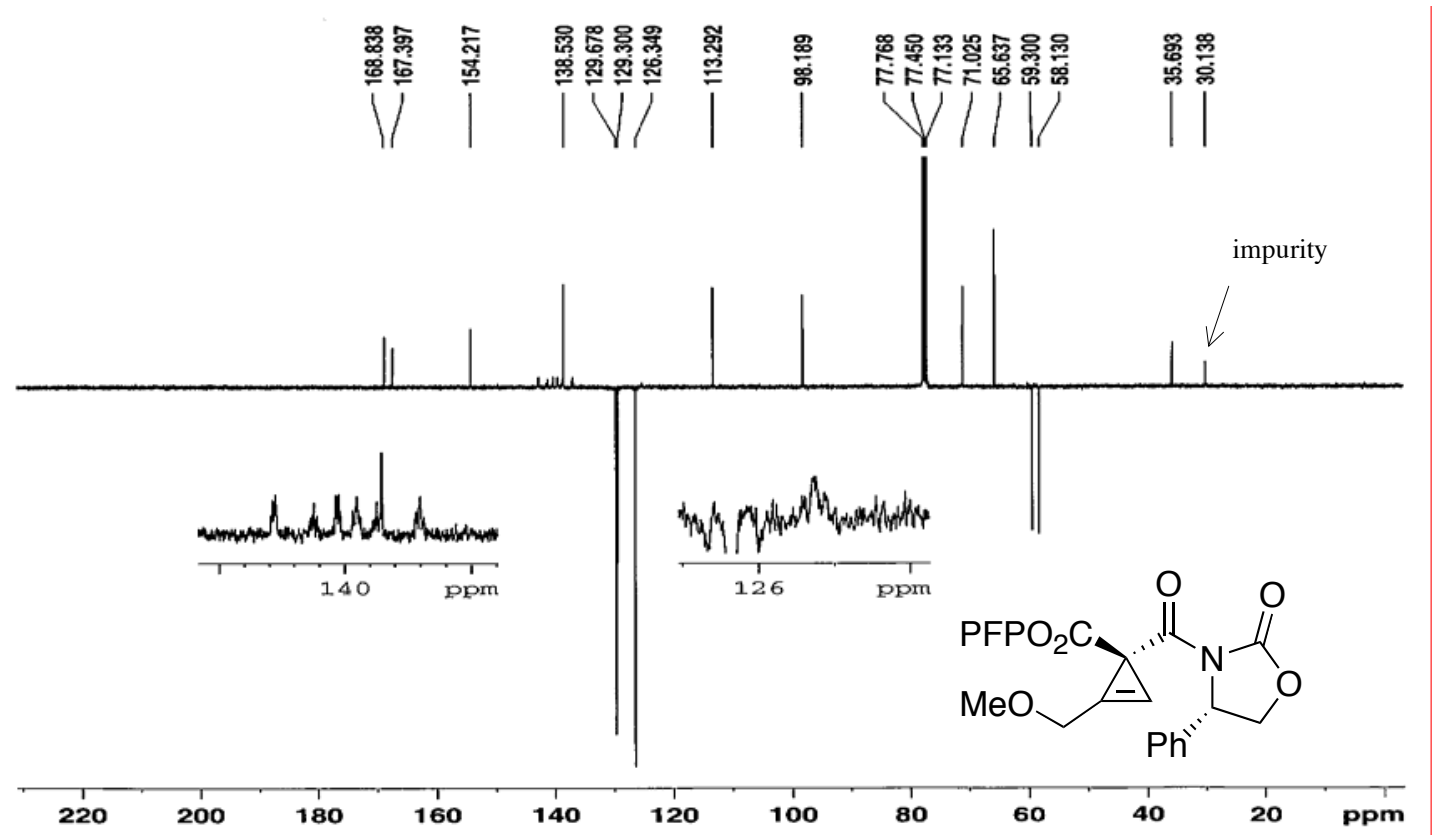


${ }^{1} \mathrm{H}$ NMR spectrum of $\mathbf{5 g}$ (major) (400 $\mathbf{M H z}, \mathrm{CDCl}_{3}$ )

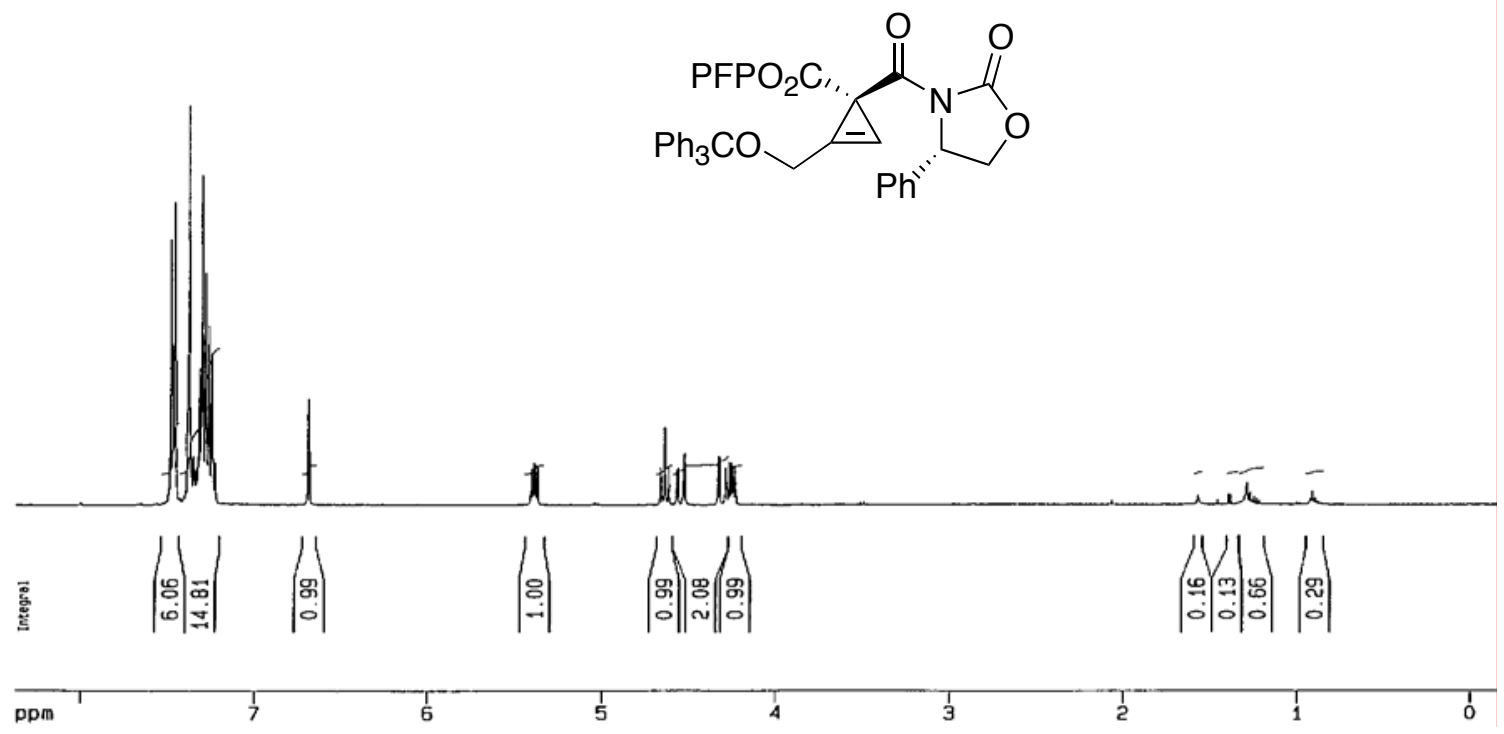

${ }^{13} \mathrm{C}$ NMR spectrum of $\mathbf{5 g}$ (major) (100 $\mathrm{MHz}, \mathrm{CDCl}_{3}$ )

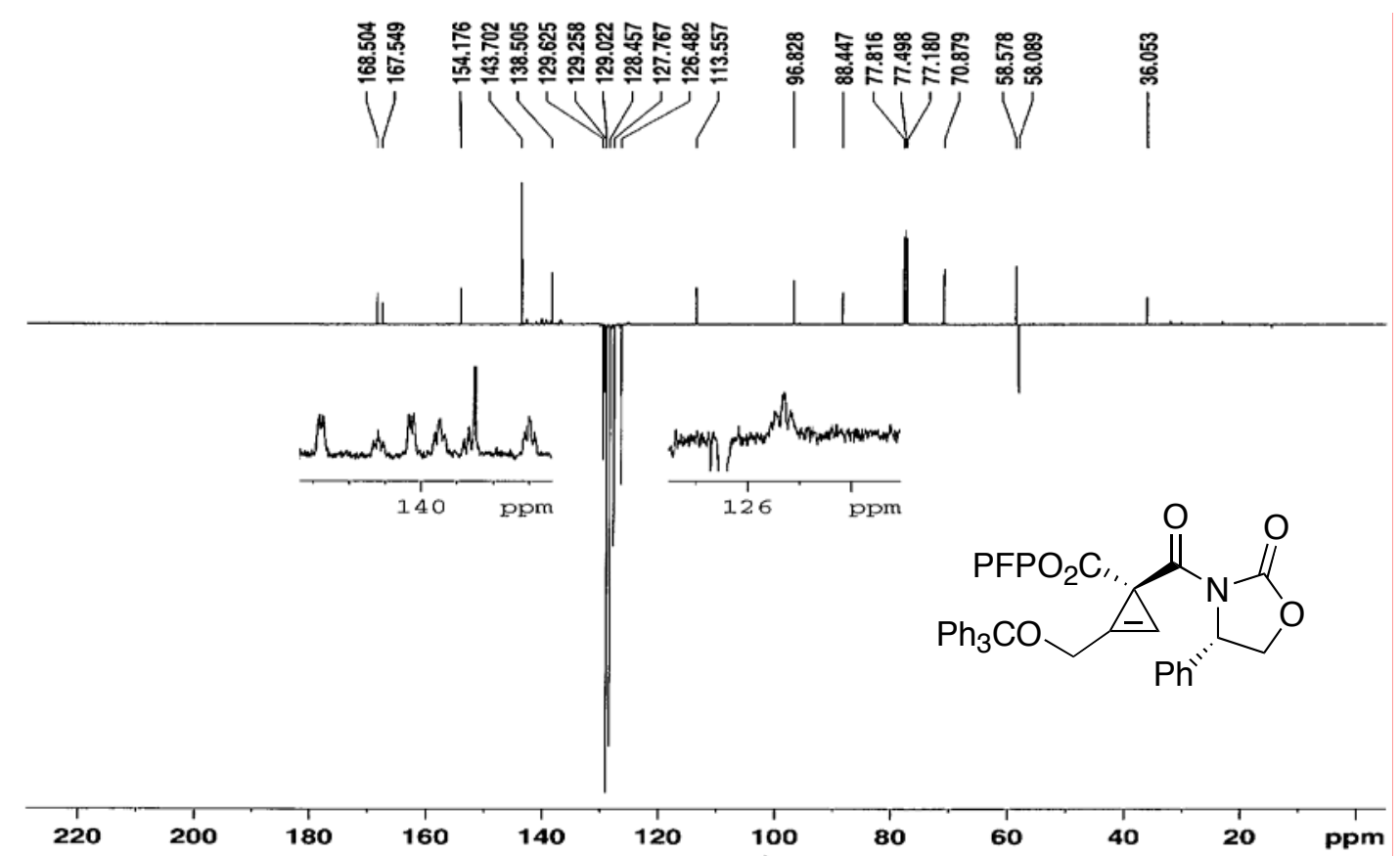


${ }^{1} \mathrm{H}$ NMR spectrum of $\mathbf{5 g}$ (minor) (400 $\mathbf{M H z}, \mathrm{CDCl}_{3}, \sim 90 \%$ purity)
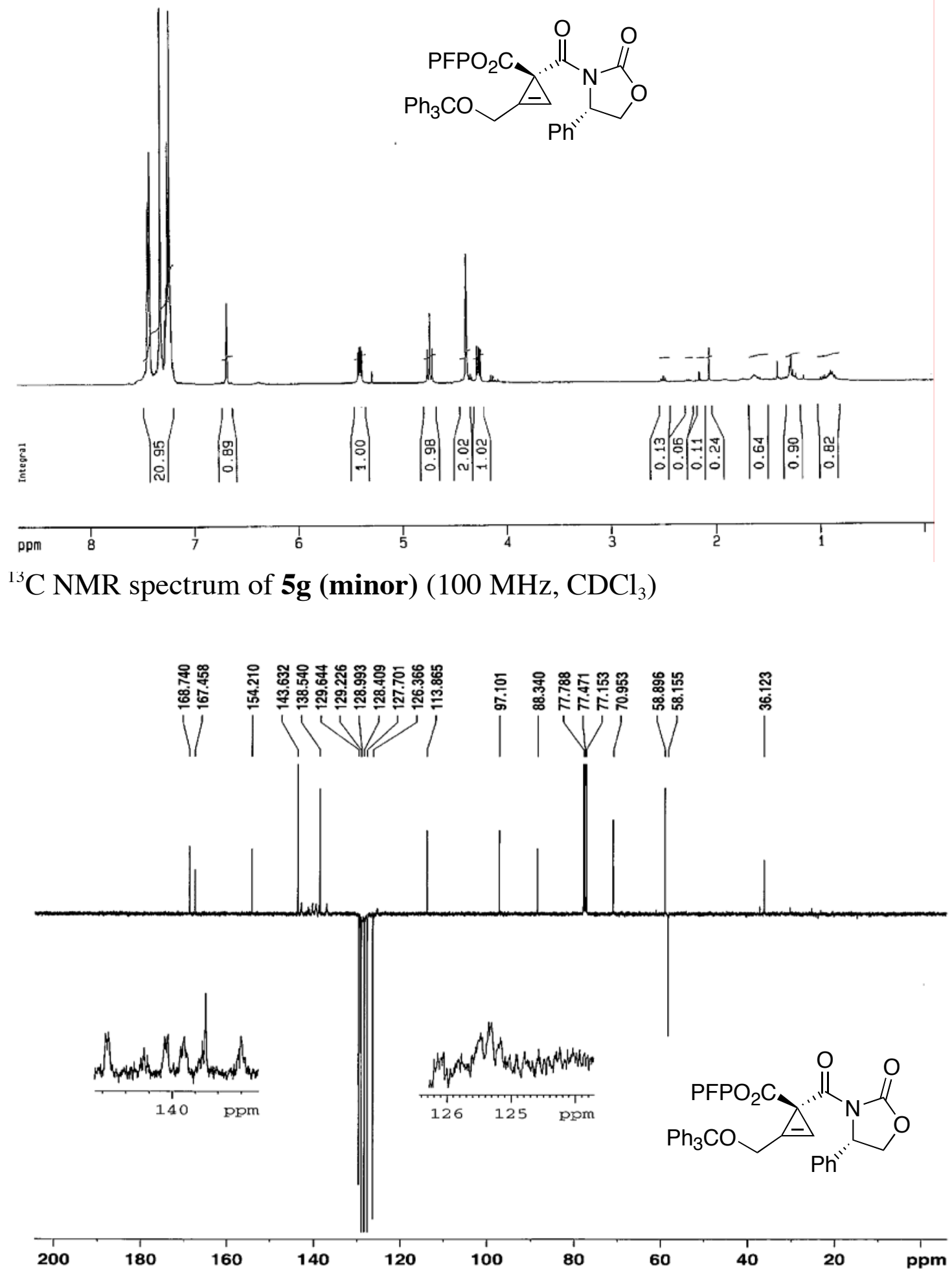
${ }^{1} \mathrm{H}$ NMR spectrum of $6 \mathbf{a}\left(400 \mathrm{MHz}, \mathrm{CDCl}_{3}\right)$

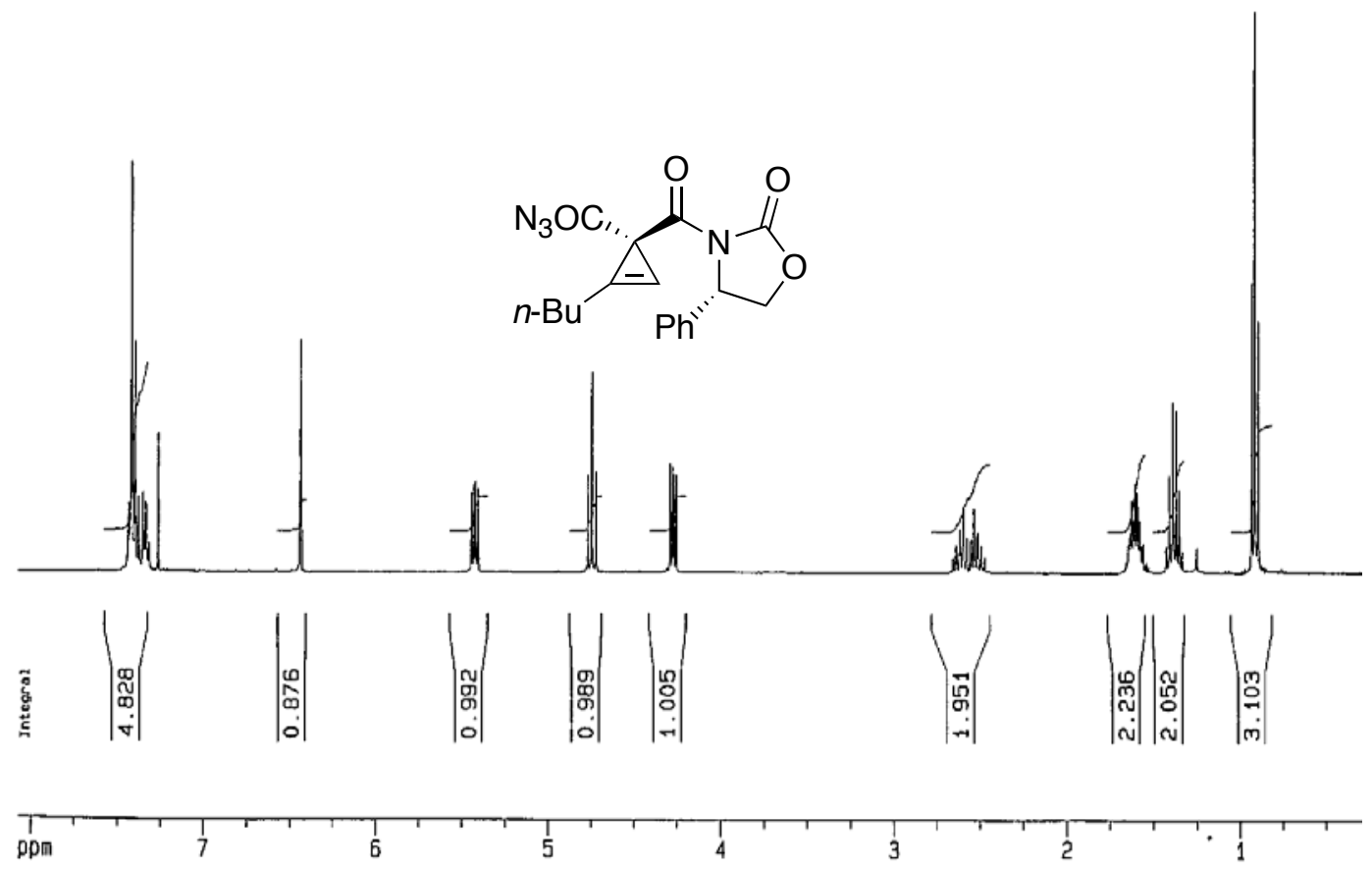

${ }^{13} \mathrm{C}$ NMR spectrum of $\mathbf{6 a}\left(100 \mathrm{MHz}, \mathrm{CDCl}_{3}\right)$

言

| | | |

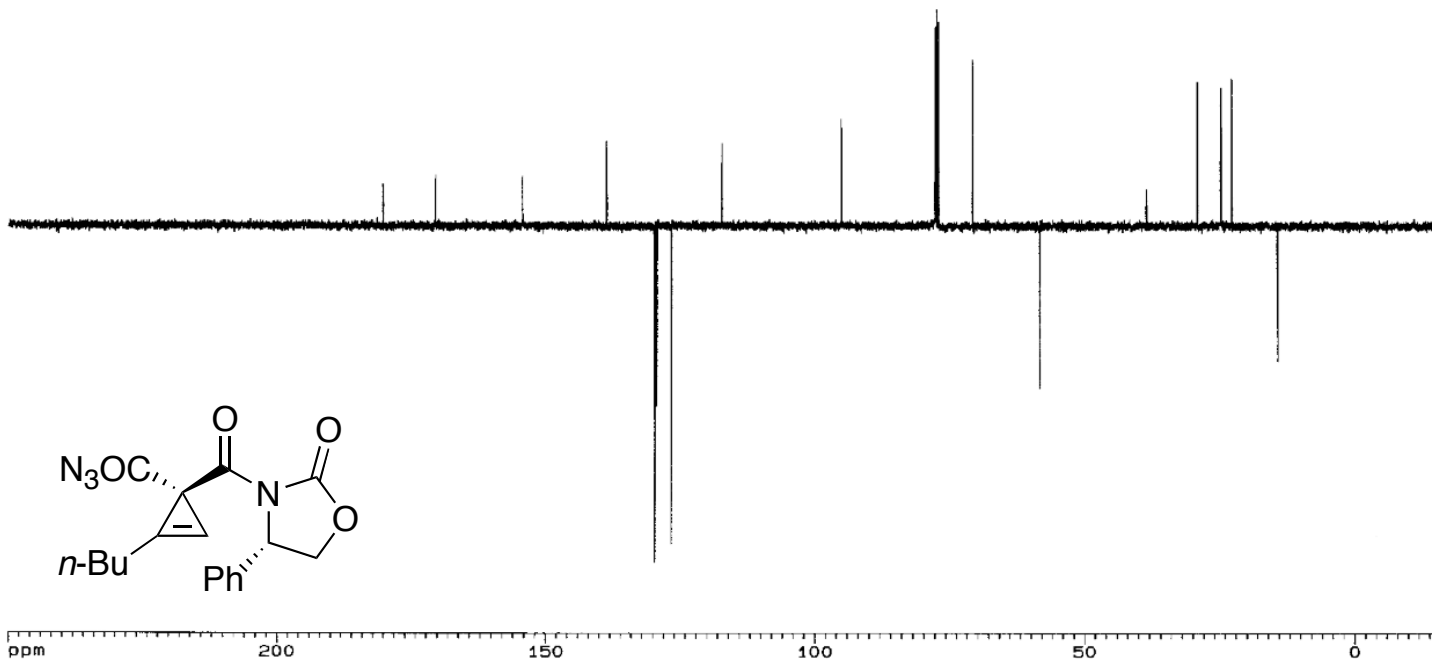


${ }^{1} \mathrm{H}$ NMR spectrum of $\mathbf{6 b}\left(400 \mathrm{MHz}, \mathrm{CDCl}_{3}\right)$

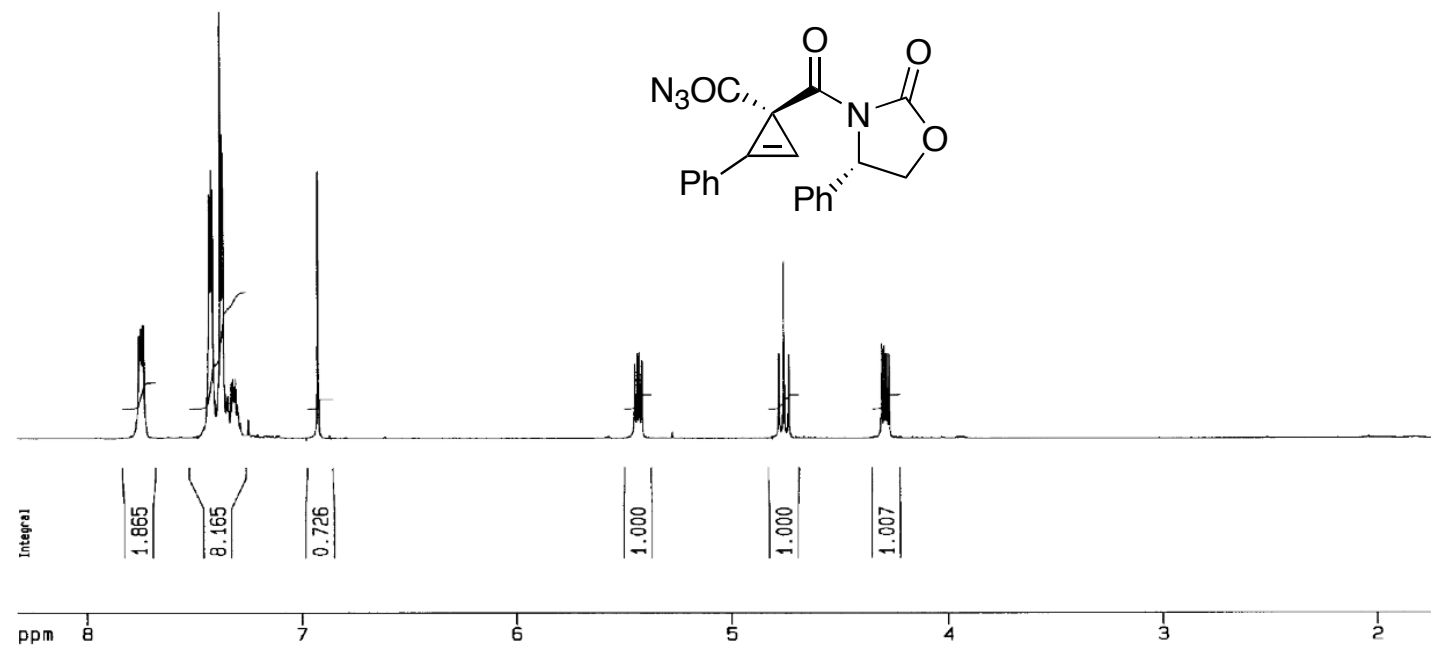

${ }^{13} \mathrm{C}$ NMR spectrum of $\mathbf{6 b}\left(100 \mathrm{MHz}, \mathrm{CDCl}_{3}\right)$
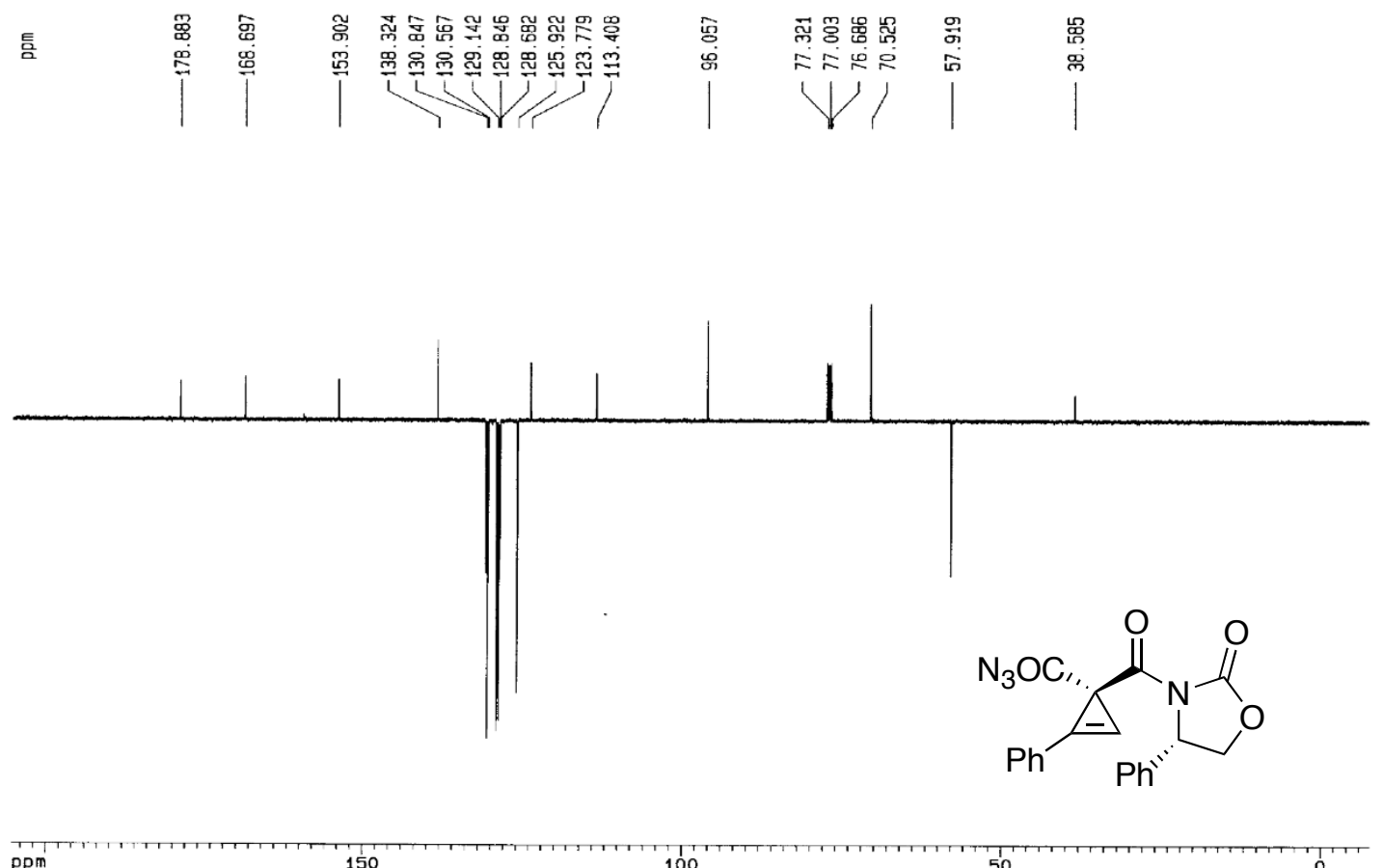
${ }^{1} \mathrm{H}$ NMR spectrum of $7 \mathbf{a}\left(400 \mathrm{MHz}, \mathrm{CDCl}_{3}\right)$

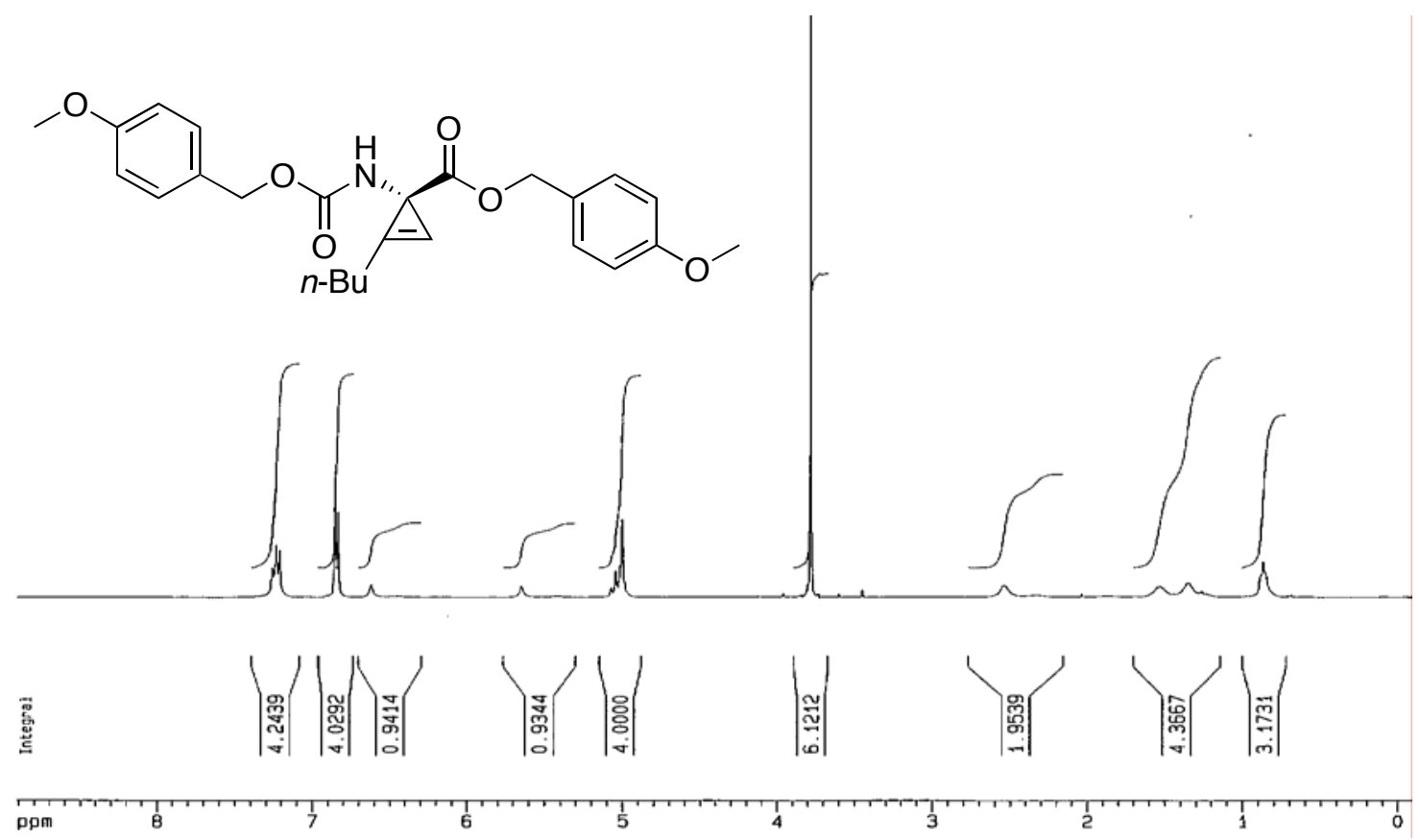

${ }^{13} \mathrm{C}$ NMR spectrum of $7 \mathbf{a}\left(100 \mathrm{MHz}, \mathrm{CDCl}_{3}\right)$

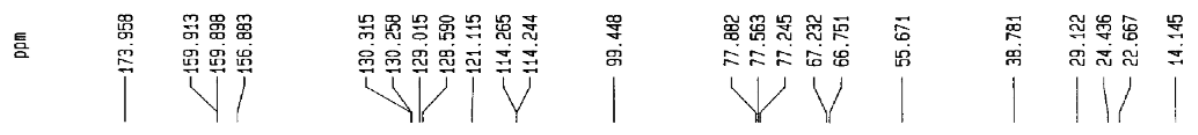

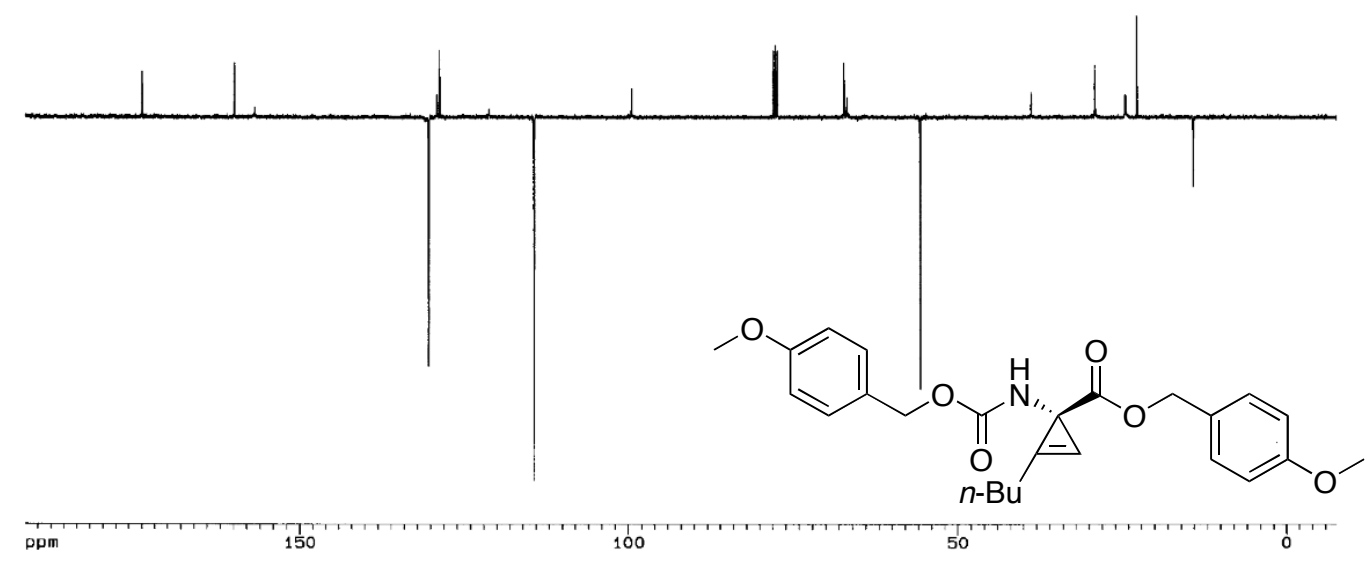


${ }^{1} \mathrm{H}$ NMR spectrum of $\mathbf{7 b}\left(400 \mathrm{MHz}, \mathrm{CDCl}_{3}\right)$

${ }^{13} \mathrm{C}$ NMR spectrum of $7 \mathbf{b}\left(100 \mathrm{MHz}, \mathrm{CDCl}_{3}\right)$

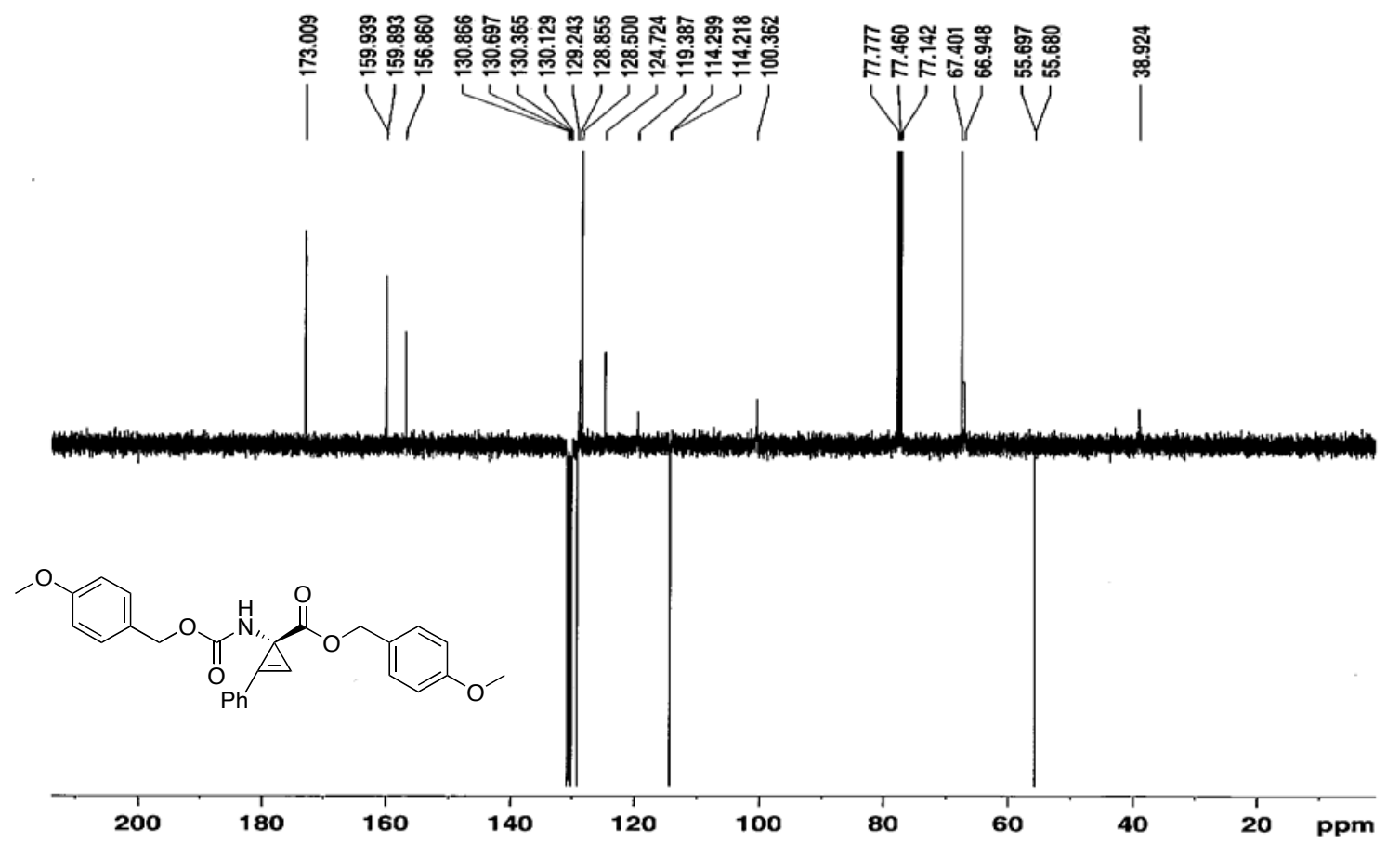


${ }^{1} \mathrm{H}$ NMR spectrum of $\mathbf{8 a}(400 \mathrm{MHz}$, dmso- $d 6,318 \mathrm{~K})$
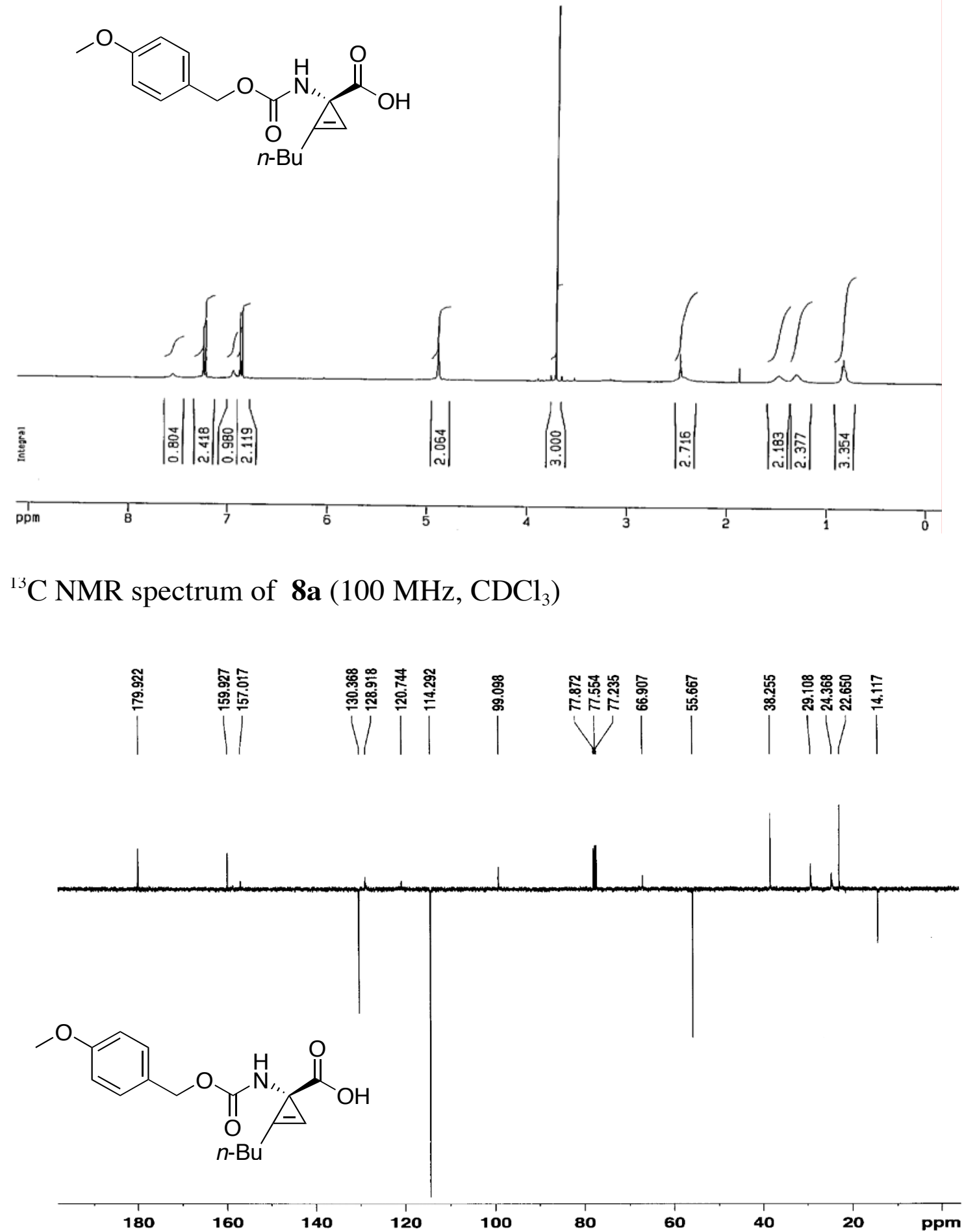
${ }^{1} \mathrm{H}$ NMR spectrum of $\mathbf{1 0 a}\left(400 \mathrm{MHz}, \mathrm{D}_{2} \mathrm{O}\right)$

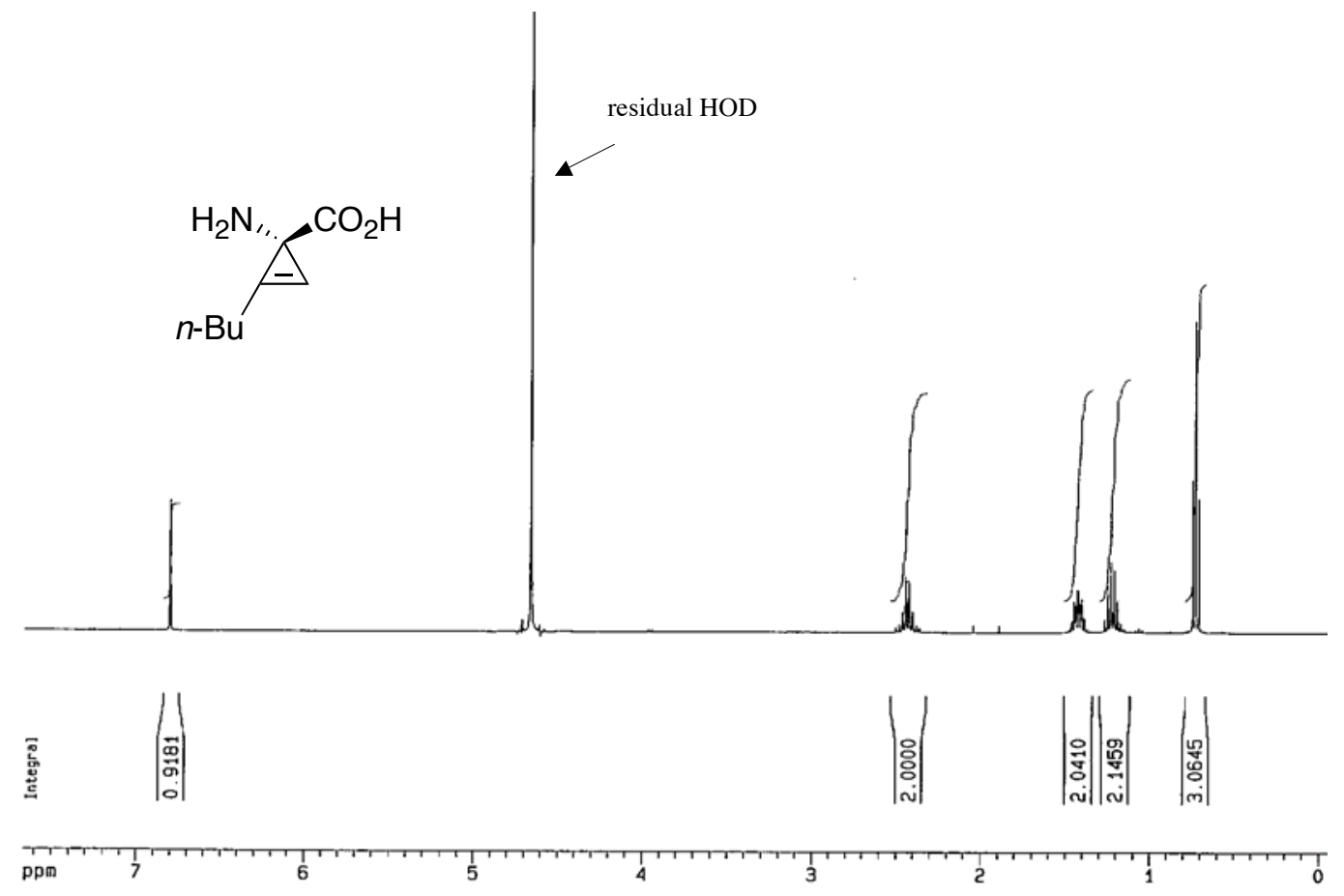

${ }^{13} \mathrm{C}$ NMR spectrum of $\mathbf{1 0 a}\left(90 \mathrm{MHz}, \mathrm{D}_{2} \mathrm{O}\right)$

흥
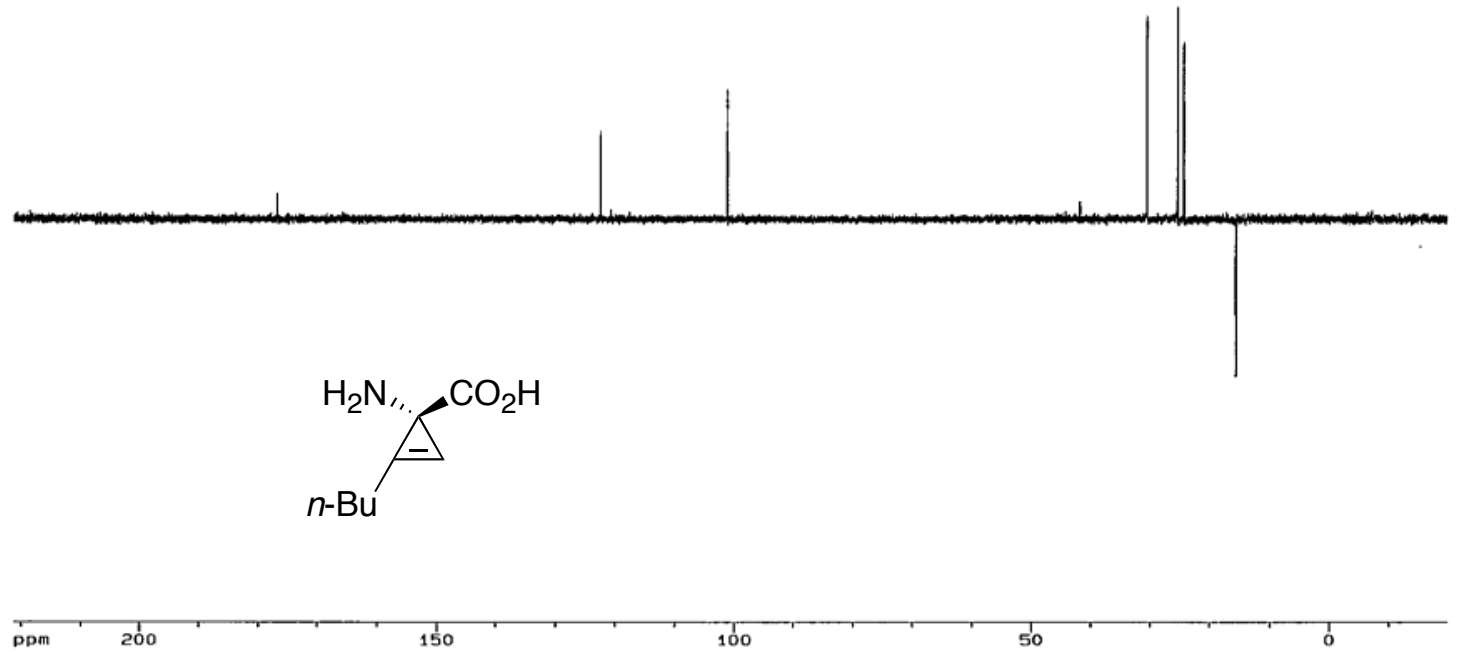
${ }^{1} \mathrm{H}$ NMR spectrum of $\mathbf{1 0 b}\left(400 \mathrm{MHz}, \mathrm{D}_{2} \mathrm{O}\right)$

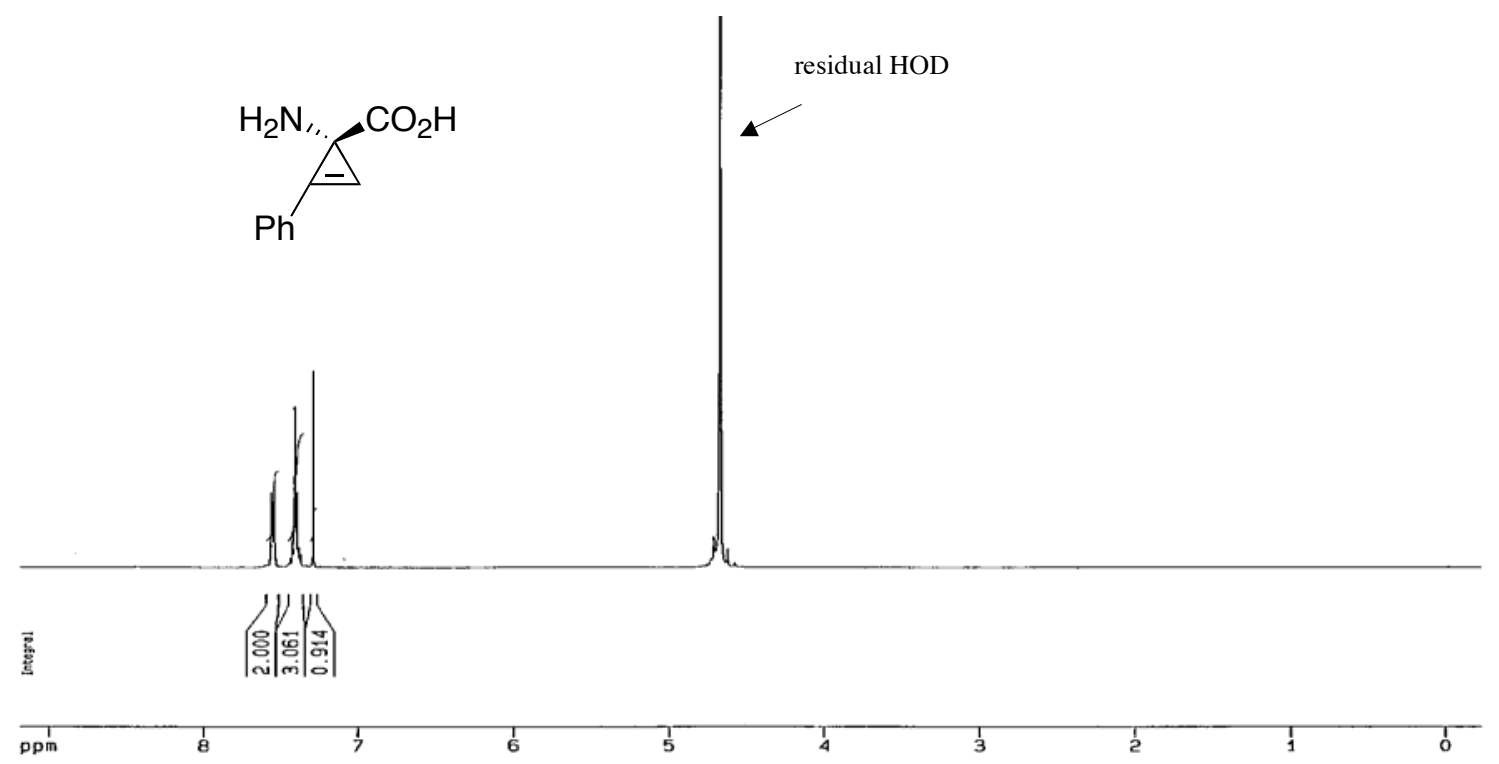

${ }^{13} \mathrm{C}$ NMR spectrum of $\mathbf{1 0 b}\left(100 \mathrm{MHz}, \mathrm{D}_{2} \mathrm{O}\right)$

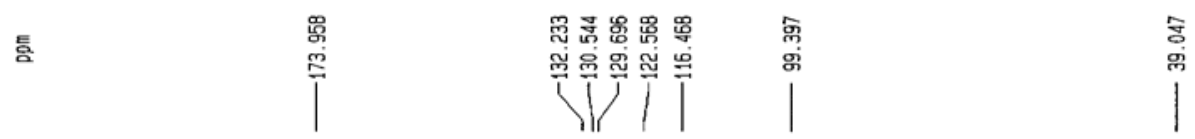

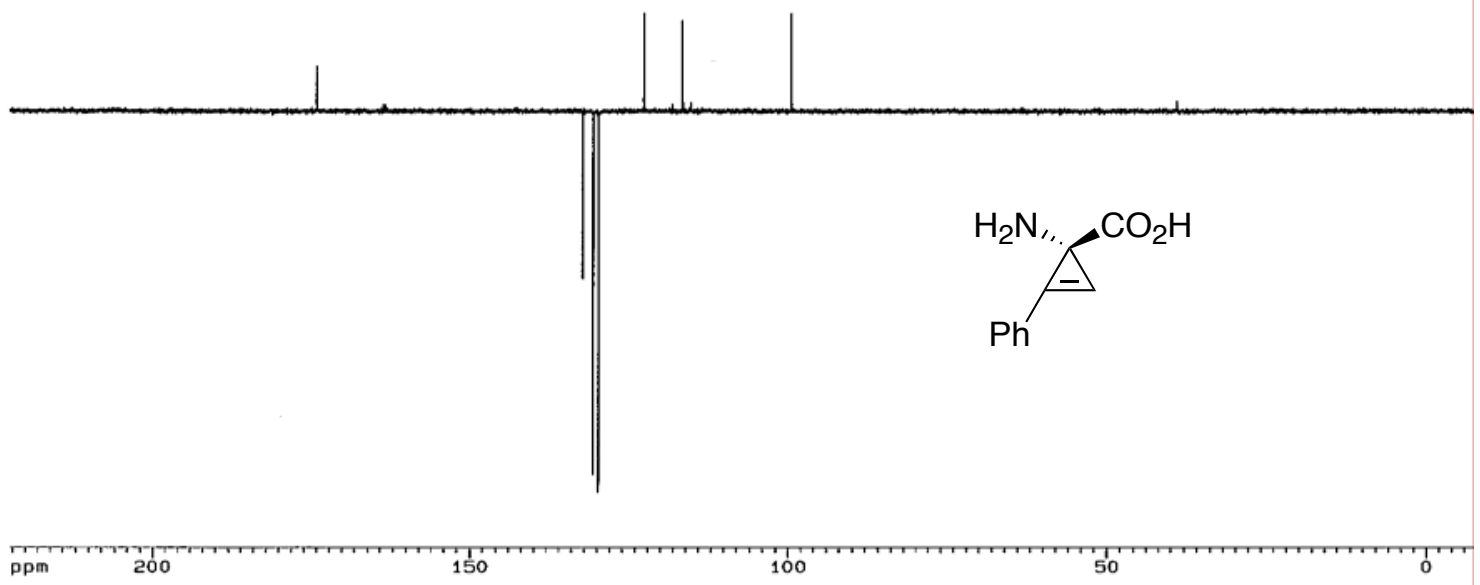


${ }^{1} \mathrm{H}$ NMR spectrum of $9 \mathbf{a}(400 \mathrm{MHz}, \mathrm{dmso}-d 6, \quad 373 \mathrm{~K})$
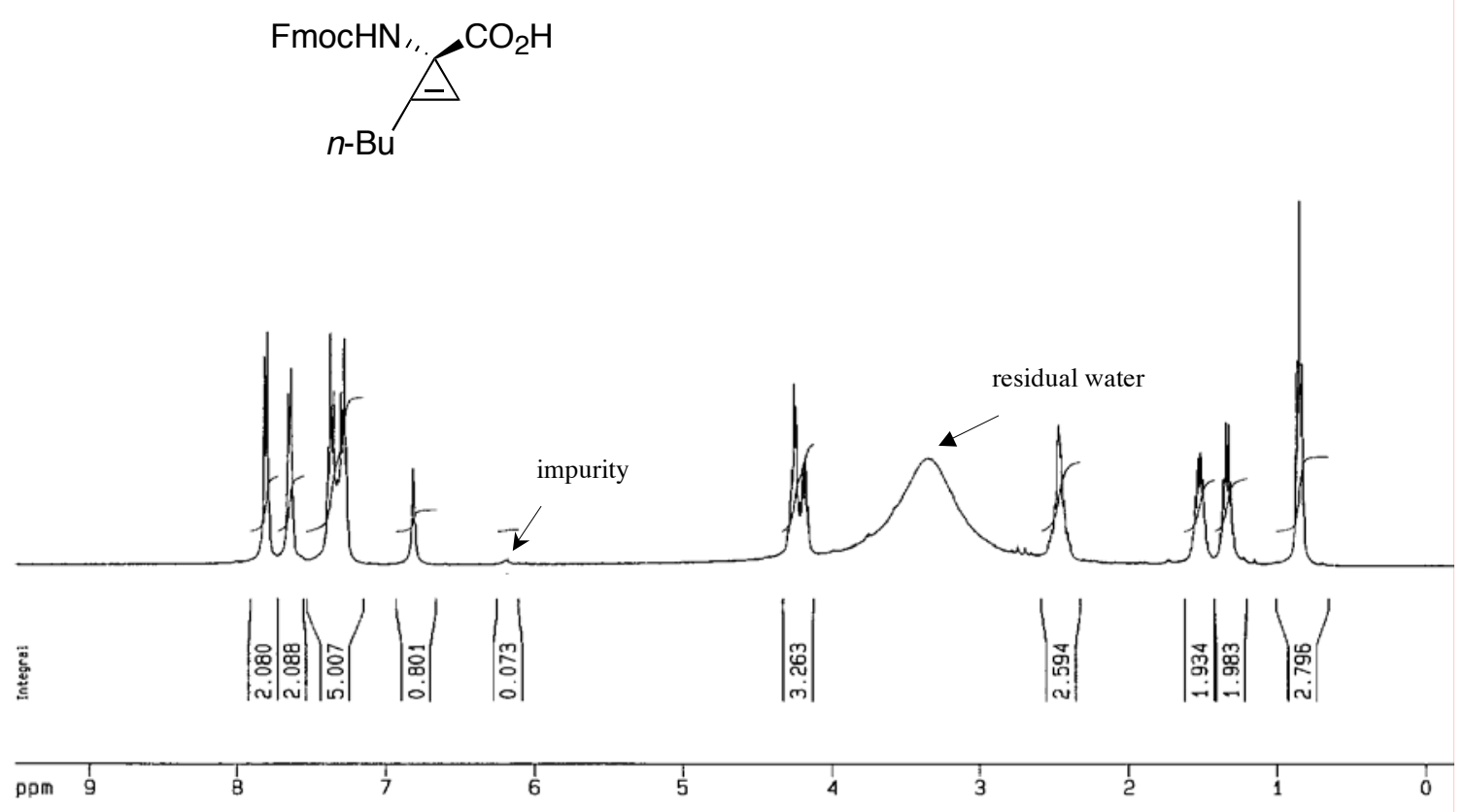

${ }^{13} \mathrm{C}$ NMR spectrum of 9a (100 MHz, dmso-d6)

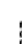

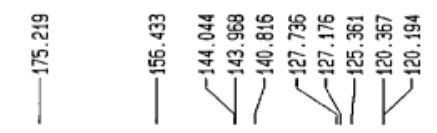

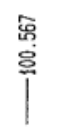

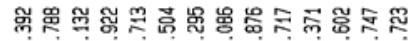

骨

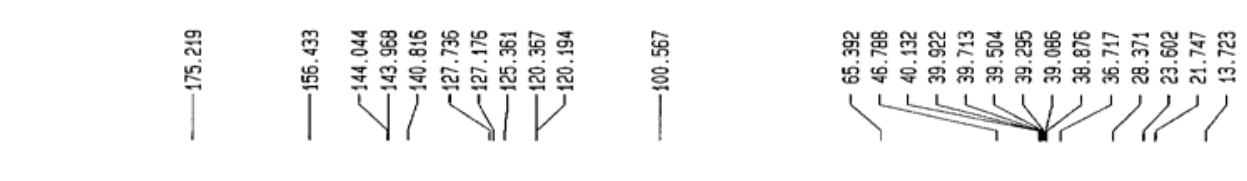

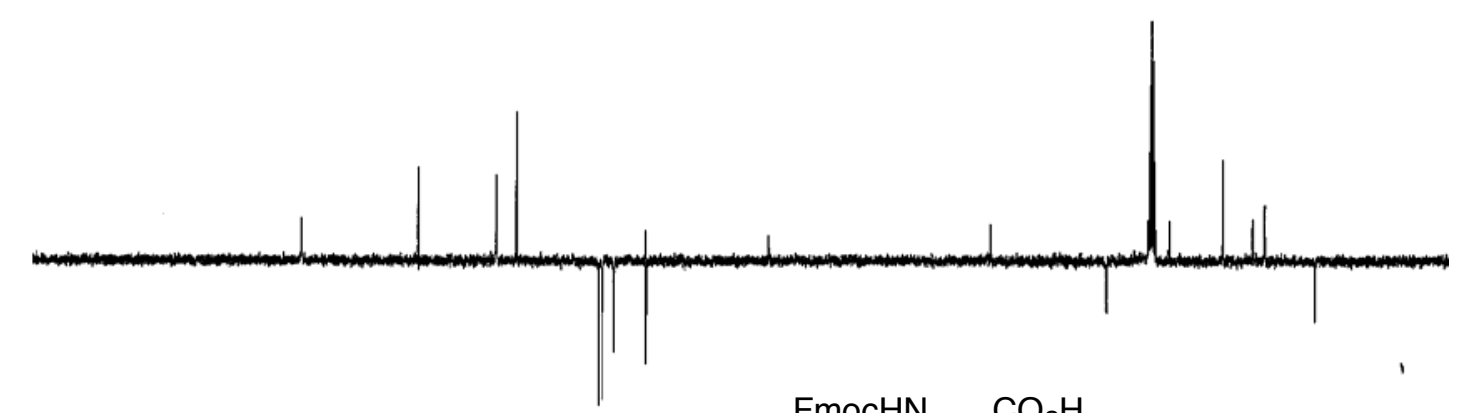

FmocHN, $\mathrm{CO}_{2} \mathrm{H}$

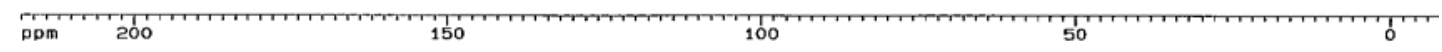


${ }^{1} \mathrm{H}$ NMR spectrum at variable temperatures of $9 \mathbf{a}(400 \mathrm{MHz}, \mathrm{dmso}-d 6)$

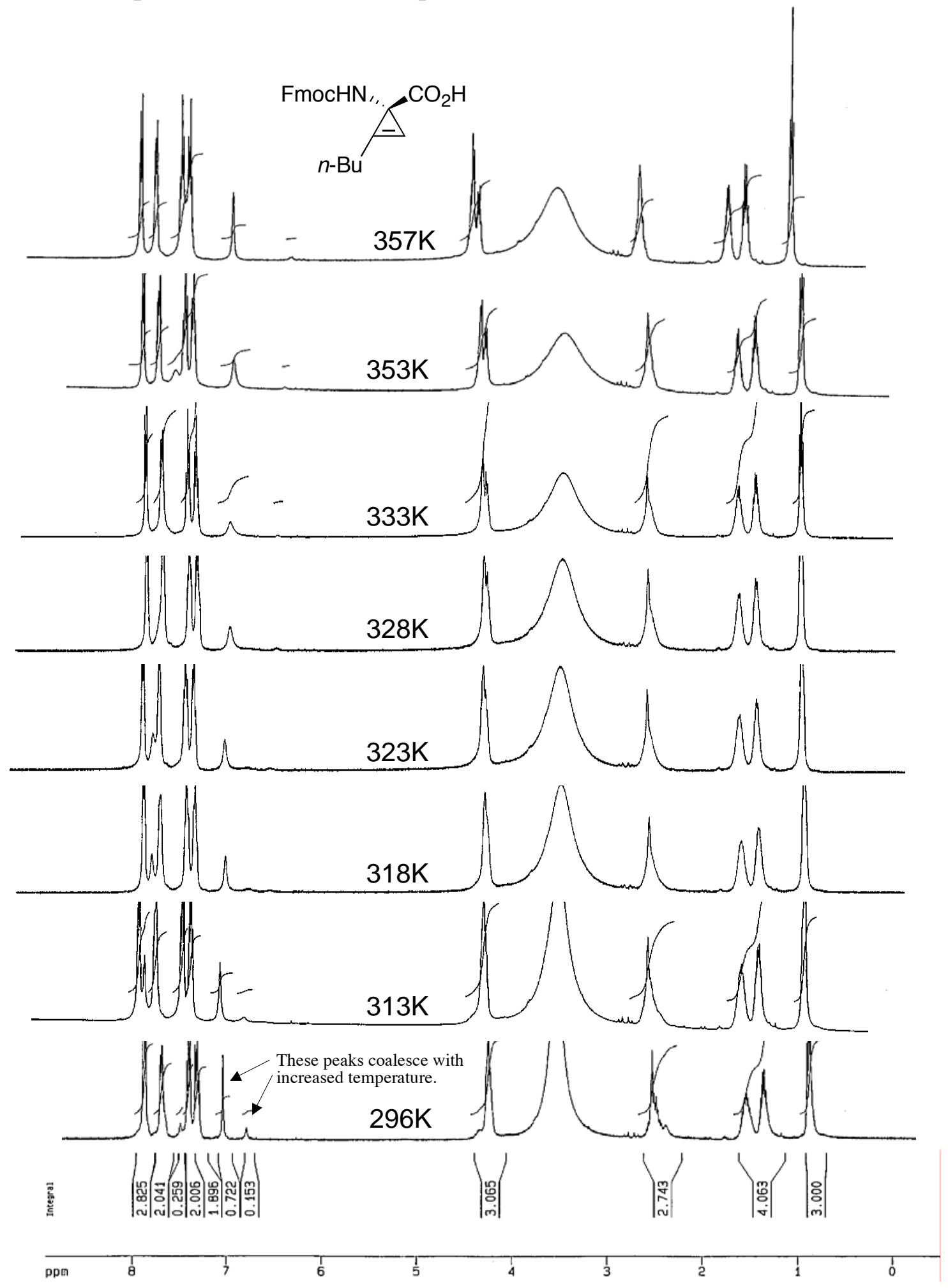


${ }^{1} \mathrm{H}$ NMR spectrum of $11\left(400 \mathrm{MHz}, \mathrm{CDCl}_{3}\right)$

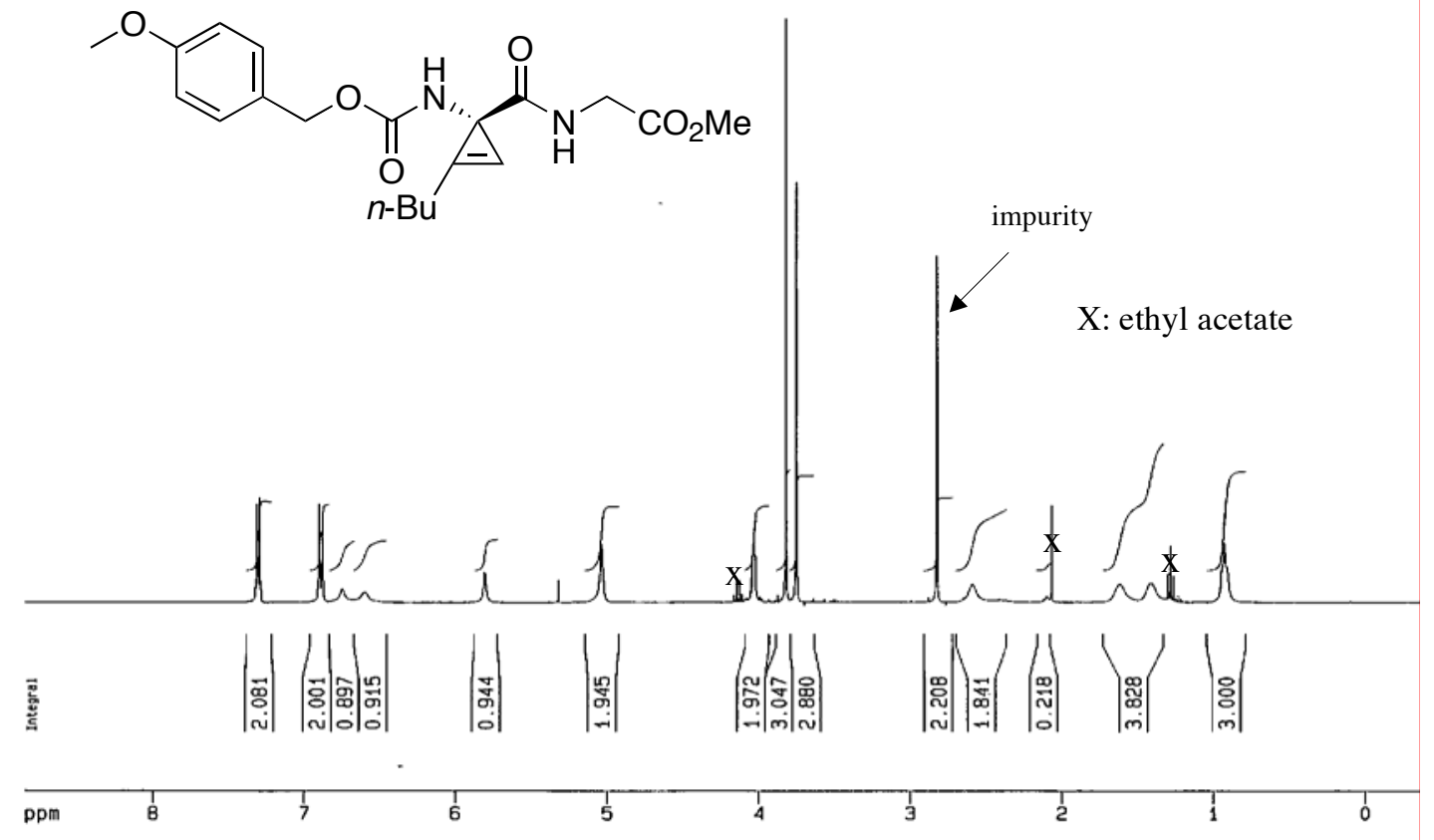

${ }^{13} \mathrm{C}$ NMR spectrum of $11\left(100 \mathrm{MHz}, \mathrm{CD}_{3} \mathrm{CN}\right)$

言
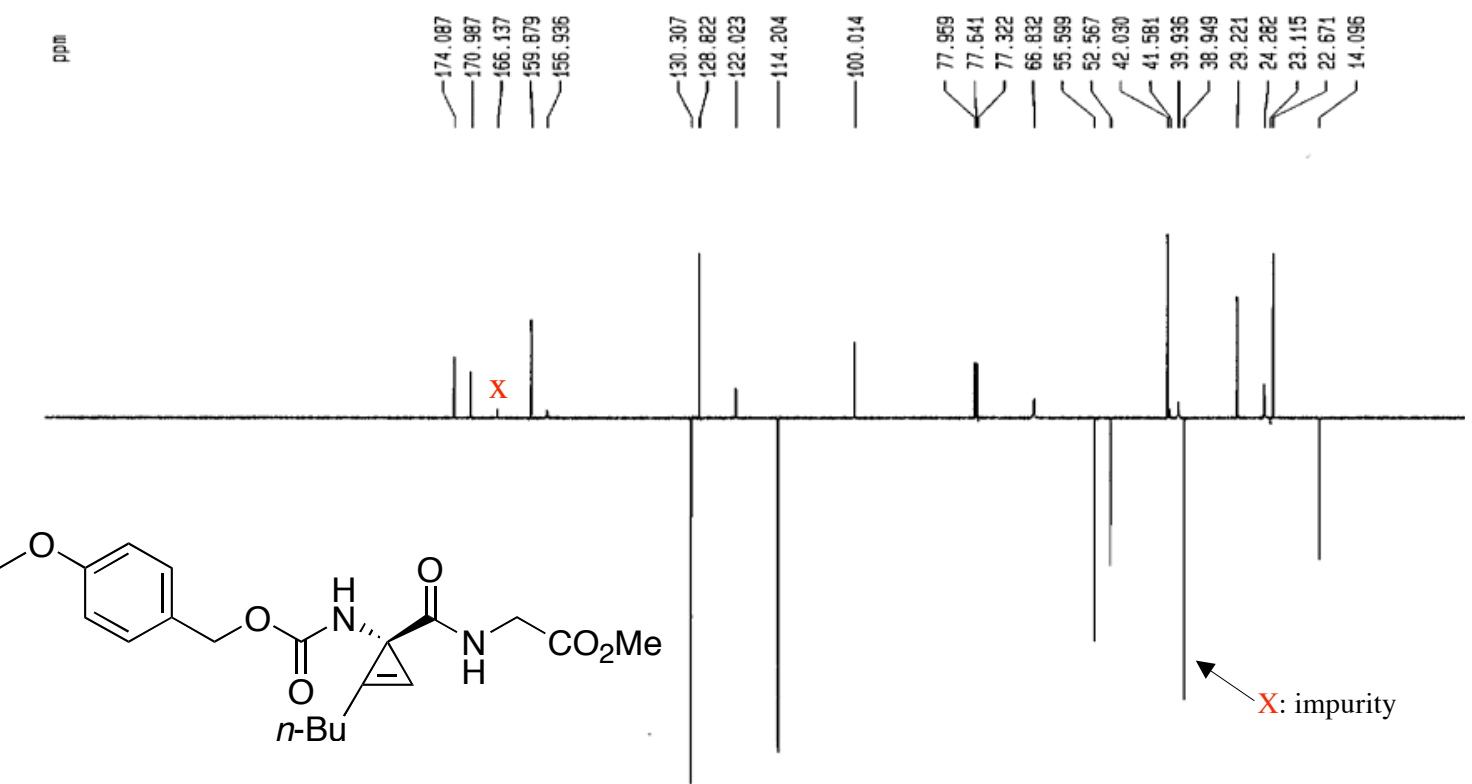

ppm

150 
${ }^{1} \mathrm{H}$ NMR spectrum of $12\left(400 \mathrm{MHz}, \mathrm{CD}_{3} \mathrm{CN}\right)$
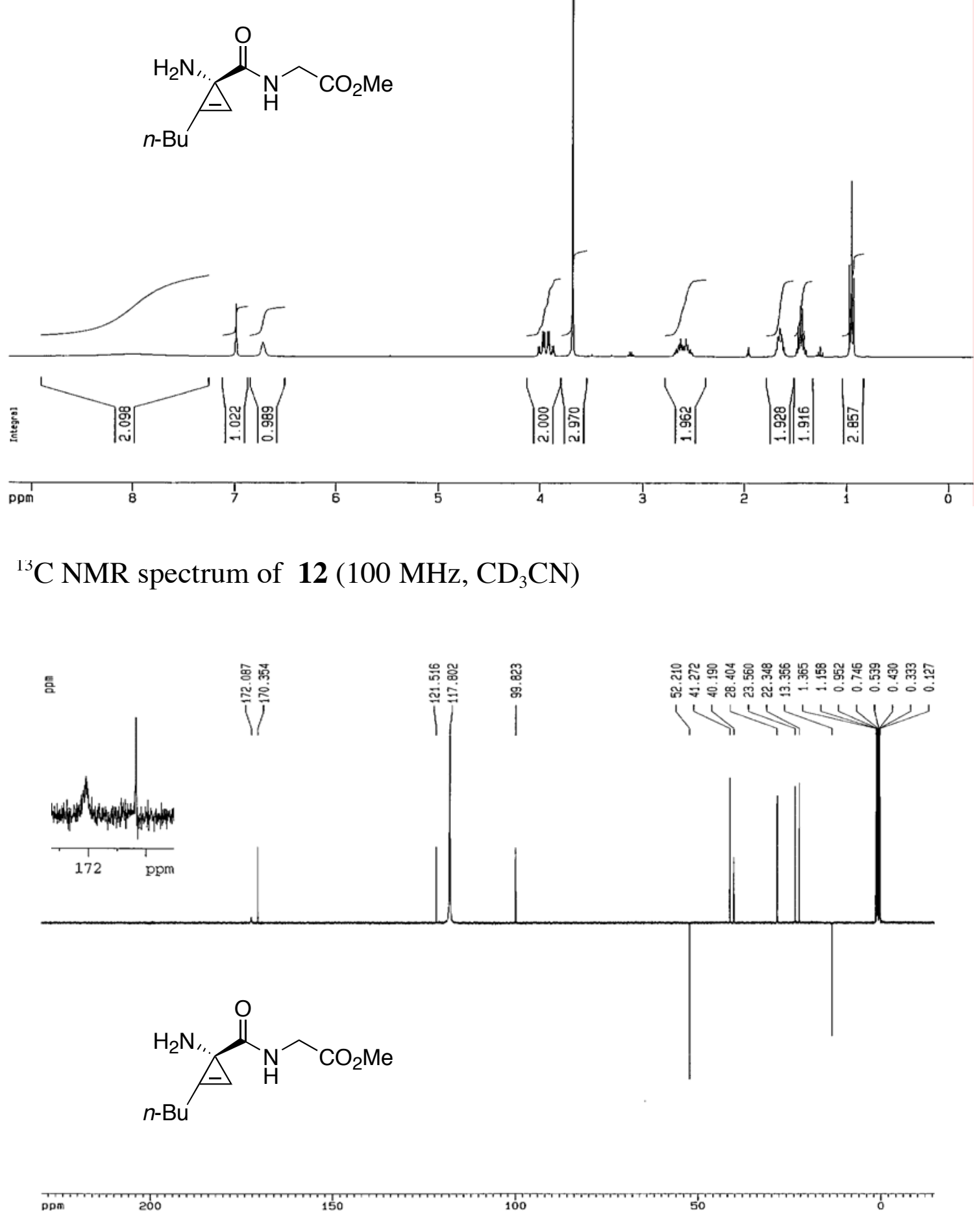
${ }^{1} \mathrm{H}$ NMR spectrum of $13\left(400 \mathrm{MHz}, \mathrm{CDCl}_{3}\right)$<smiles>CC(=O)CNC(=O)[C@]1(NC(=O)[C@@H](C)NCOF)C=C1Br</smiles>
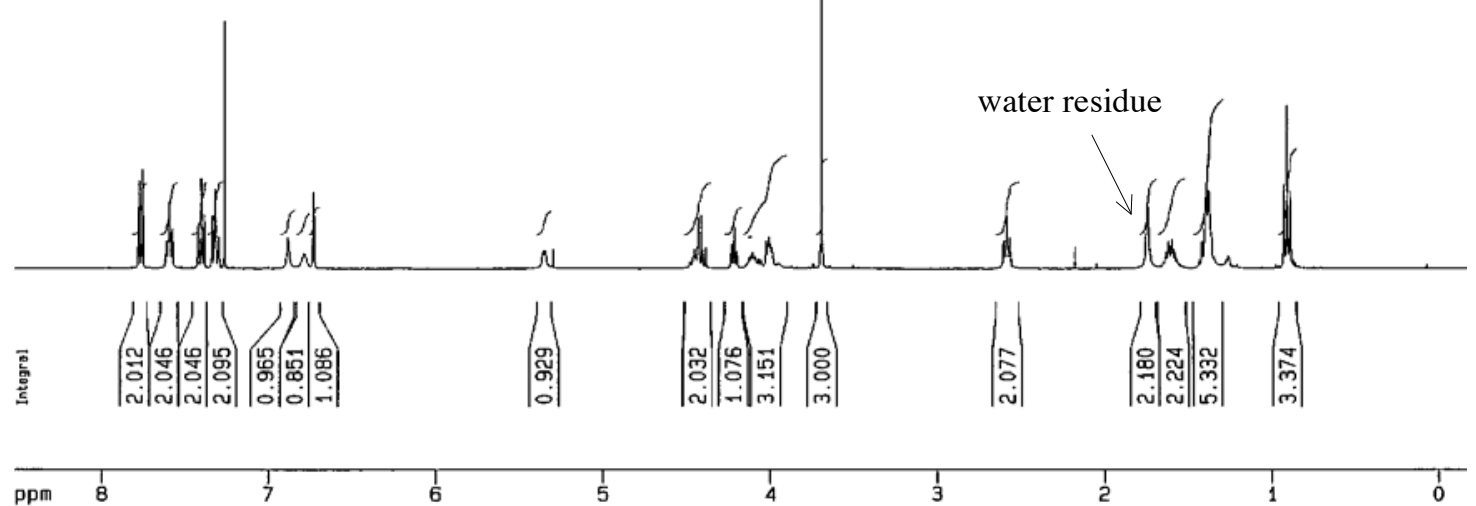

${ }^{13} \mathrm{C}$ NMR spectrum of $\mathbf{1 3}\left(100 \mathrm{MHz}, \mathrm{CDCl}_{3}\right)$

佥

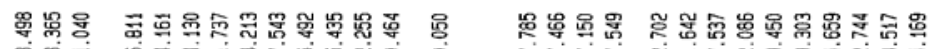

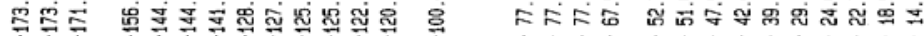

ij

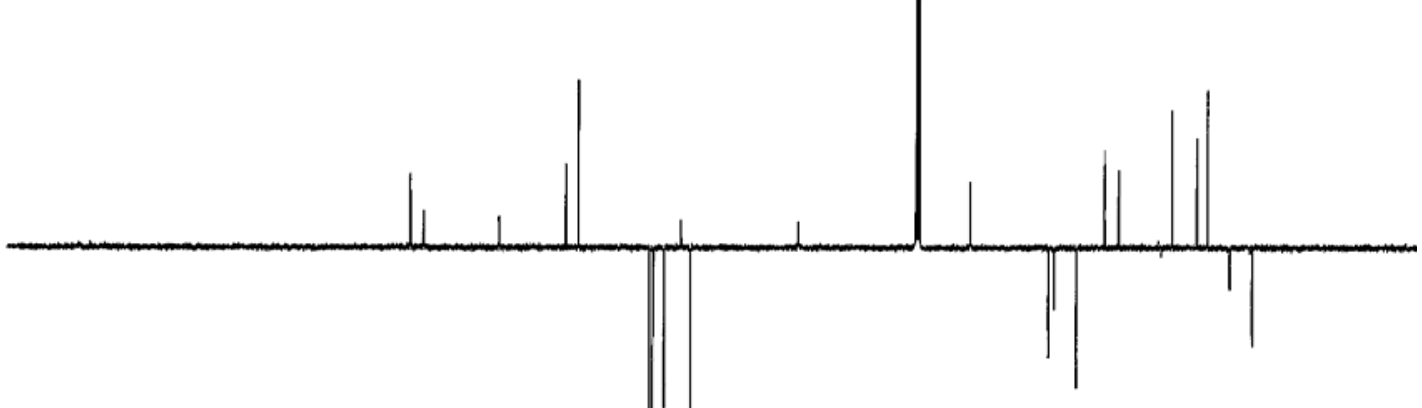

FmochN

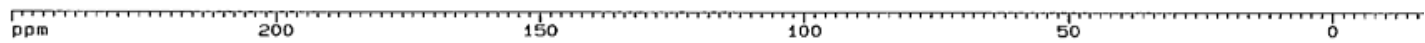


${ }^{1} \mathrm{H}$ NMR spectrum of $\mathbf{1 4}\left(400 \mathrm{MHz}, \mathrm{CD}_{3} \mathrm{CN}\right)$

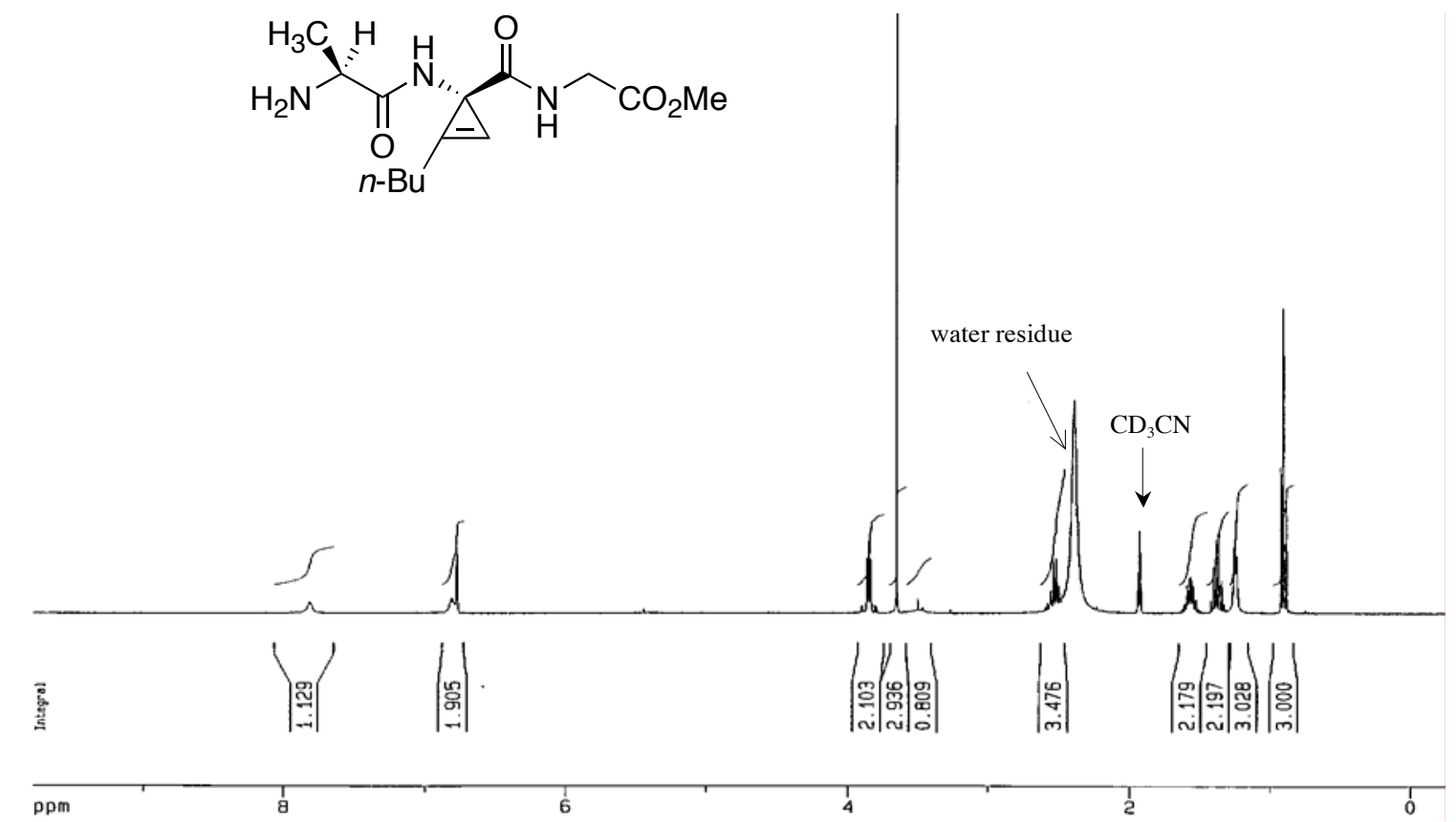

${ }^{15} \mathrm{C}$ NMR spectrum of $\mathbf{1 4}\left(100 \mathrm{MHz}, \mathrm{CDCl}_{3}\right)$
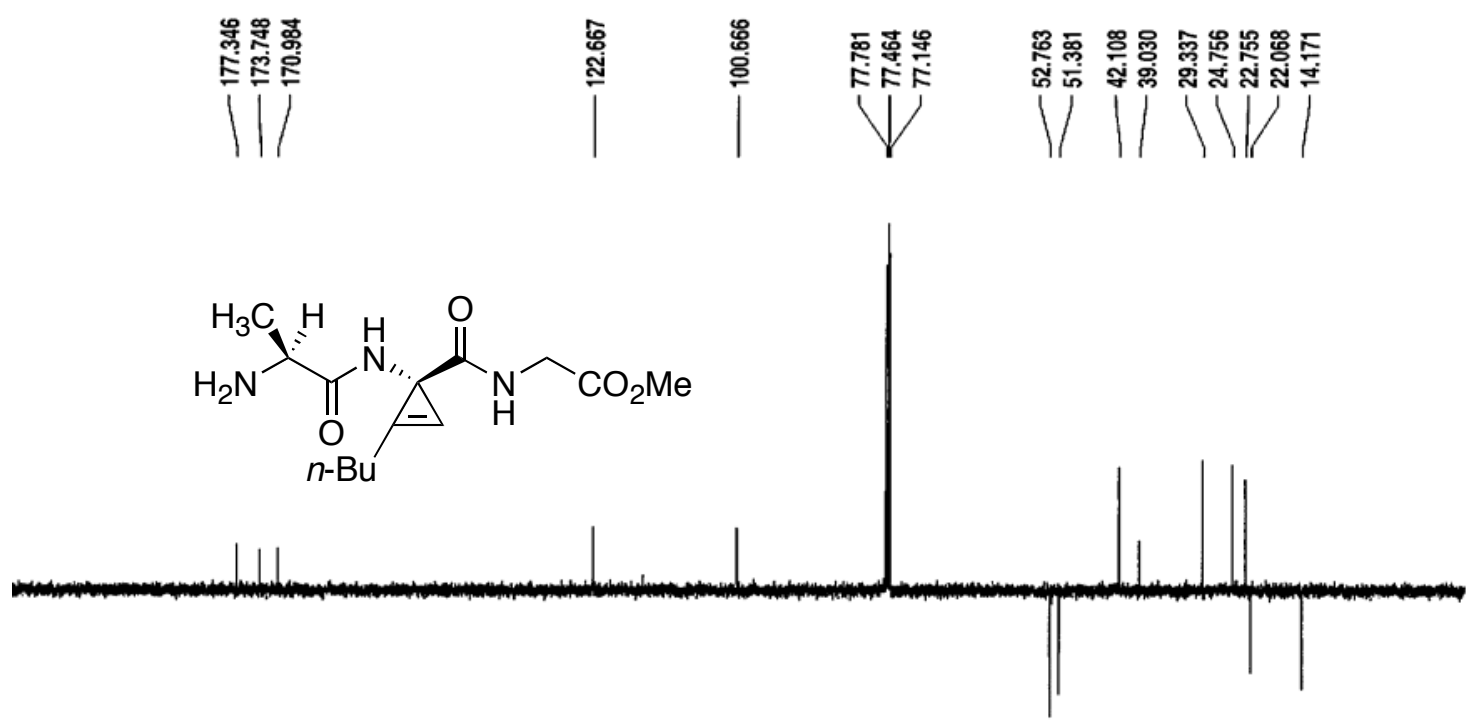

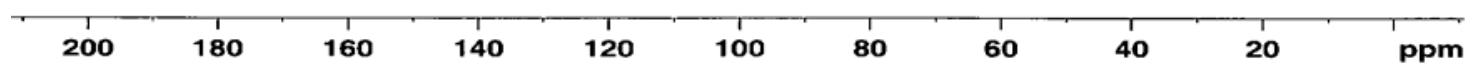


HPLC data for $\mathbf{5 a}$ :
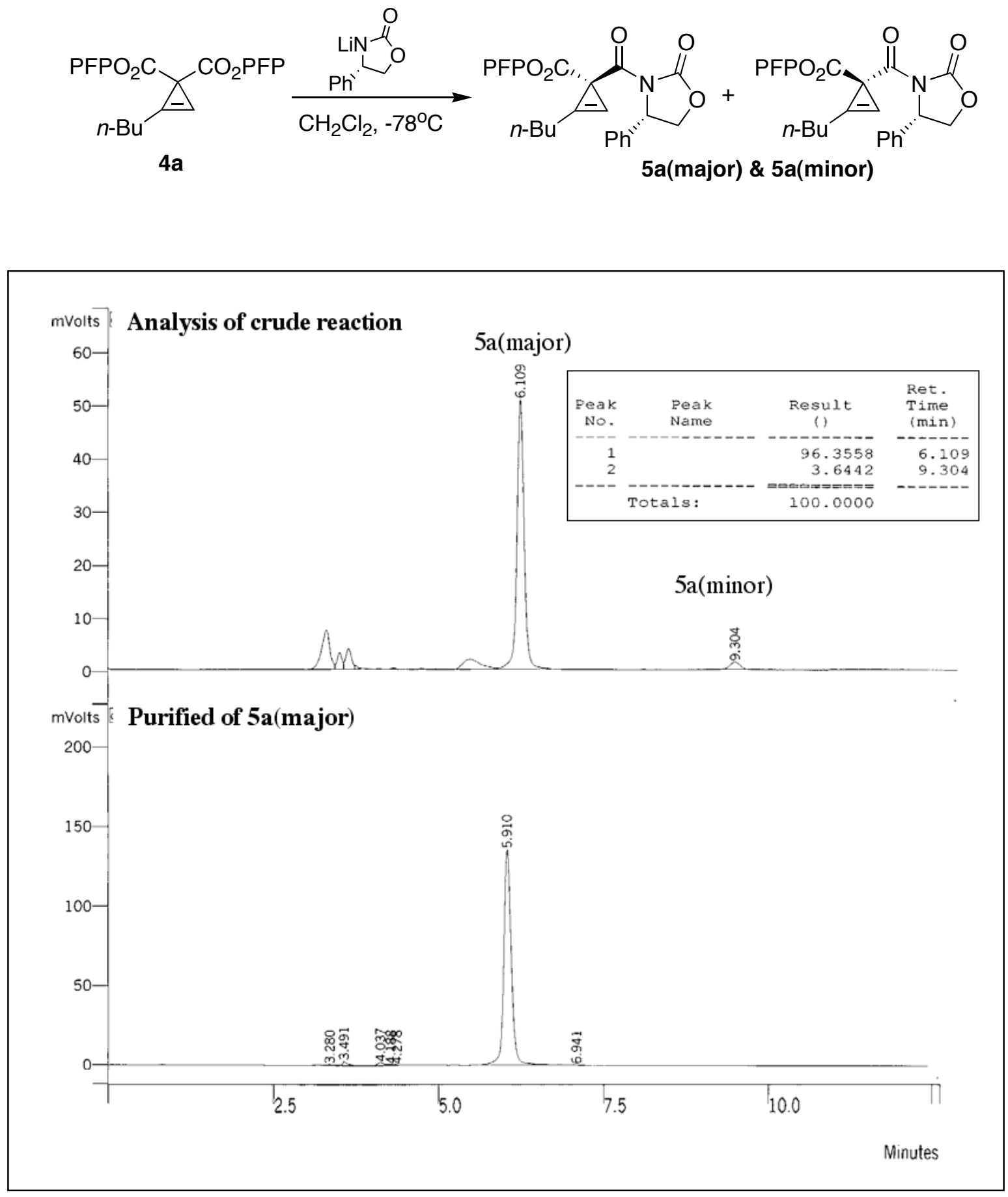
HPLC data for $\mathbf{5 b}$
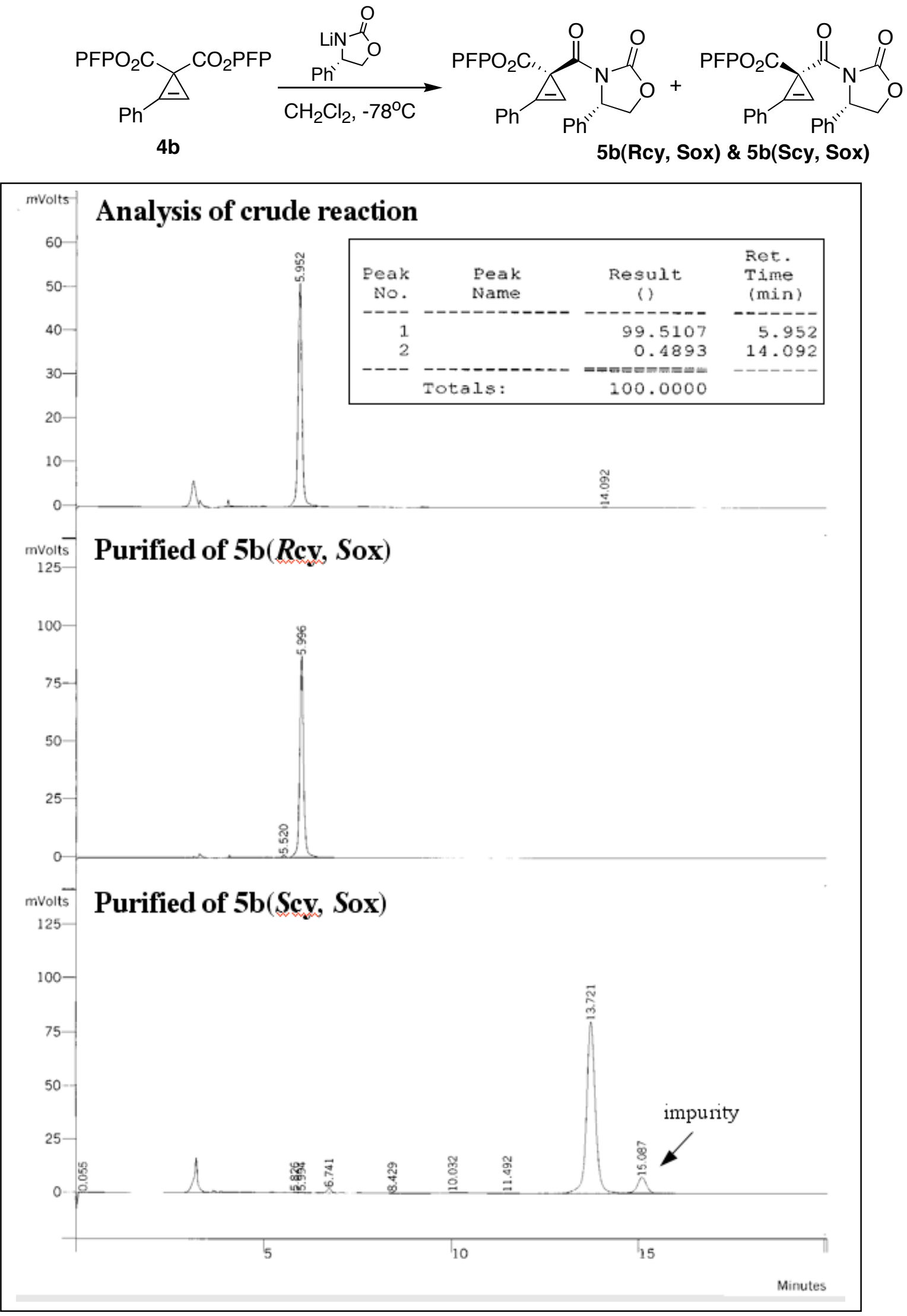
HPLC data for $\mathbf{5 c}$
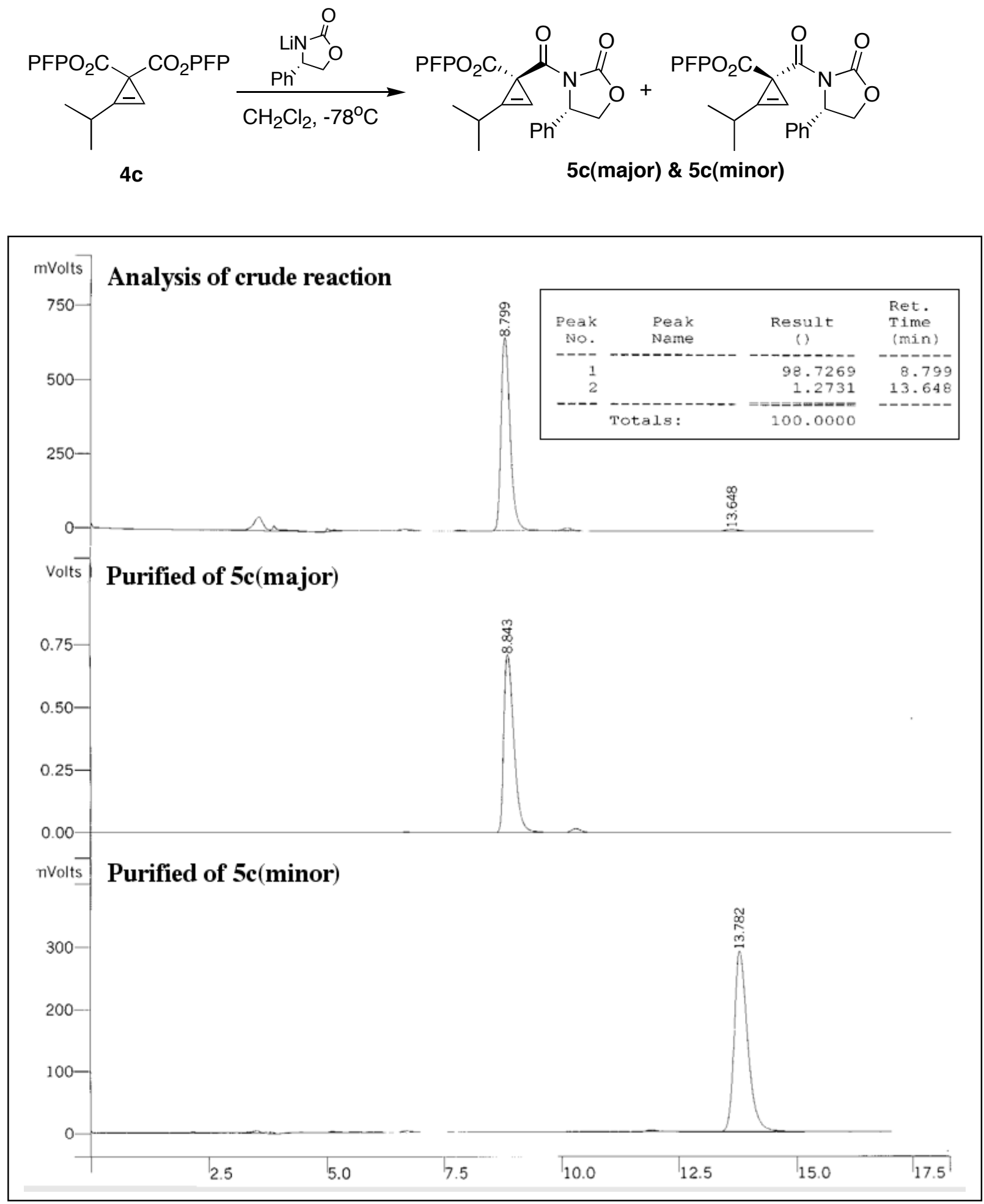
HPLC data for $\mathbf{5 d}$ :

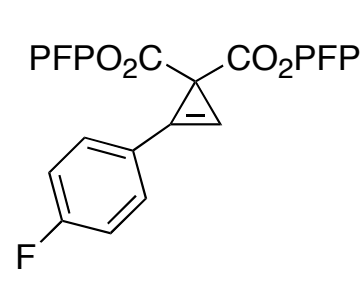

4d

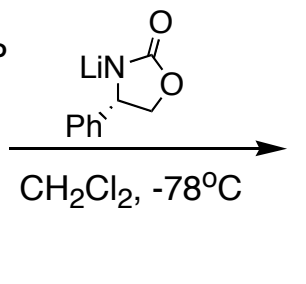

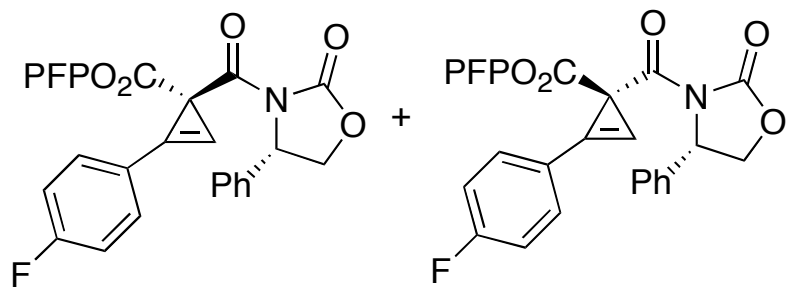

$5 d$ (major) \& $5 d$ (minor)

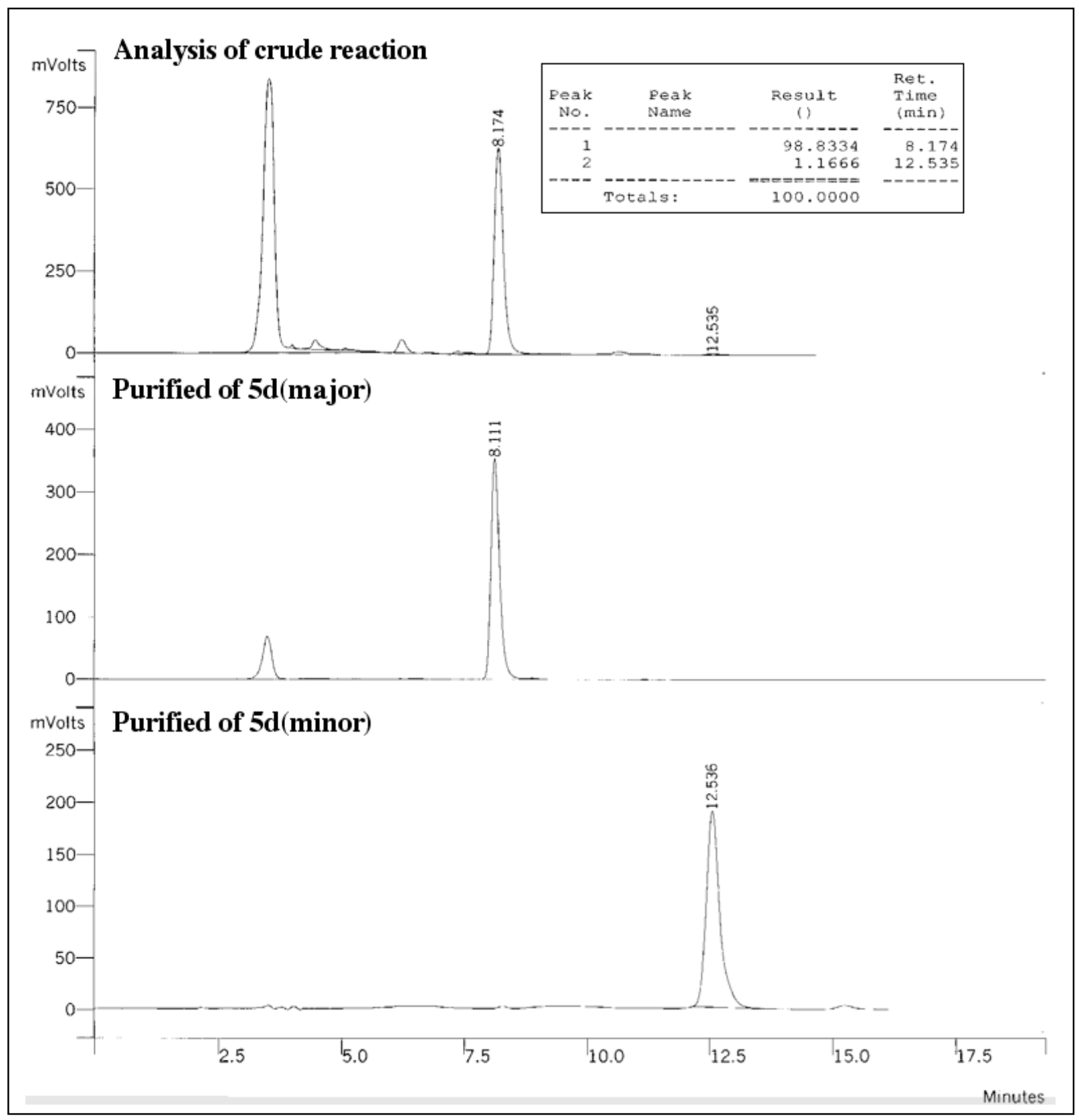


HPLC Data for 5e:
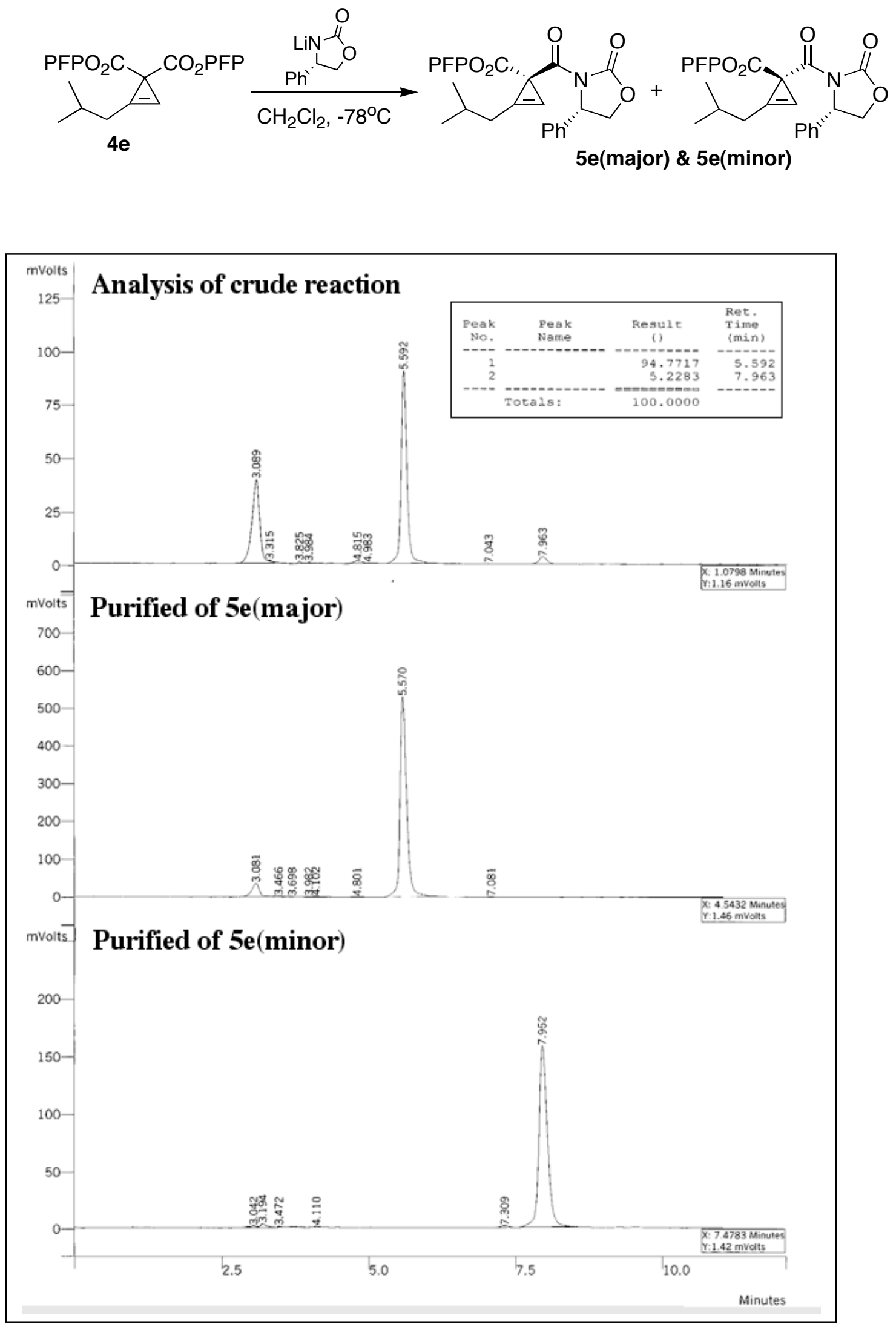
HPLC Data for $\mathbf{5 f}$
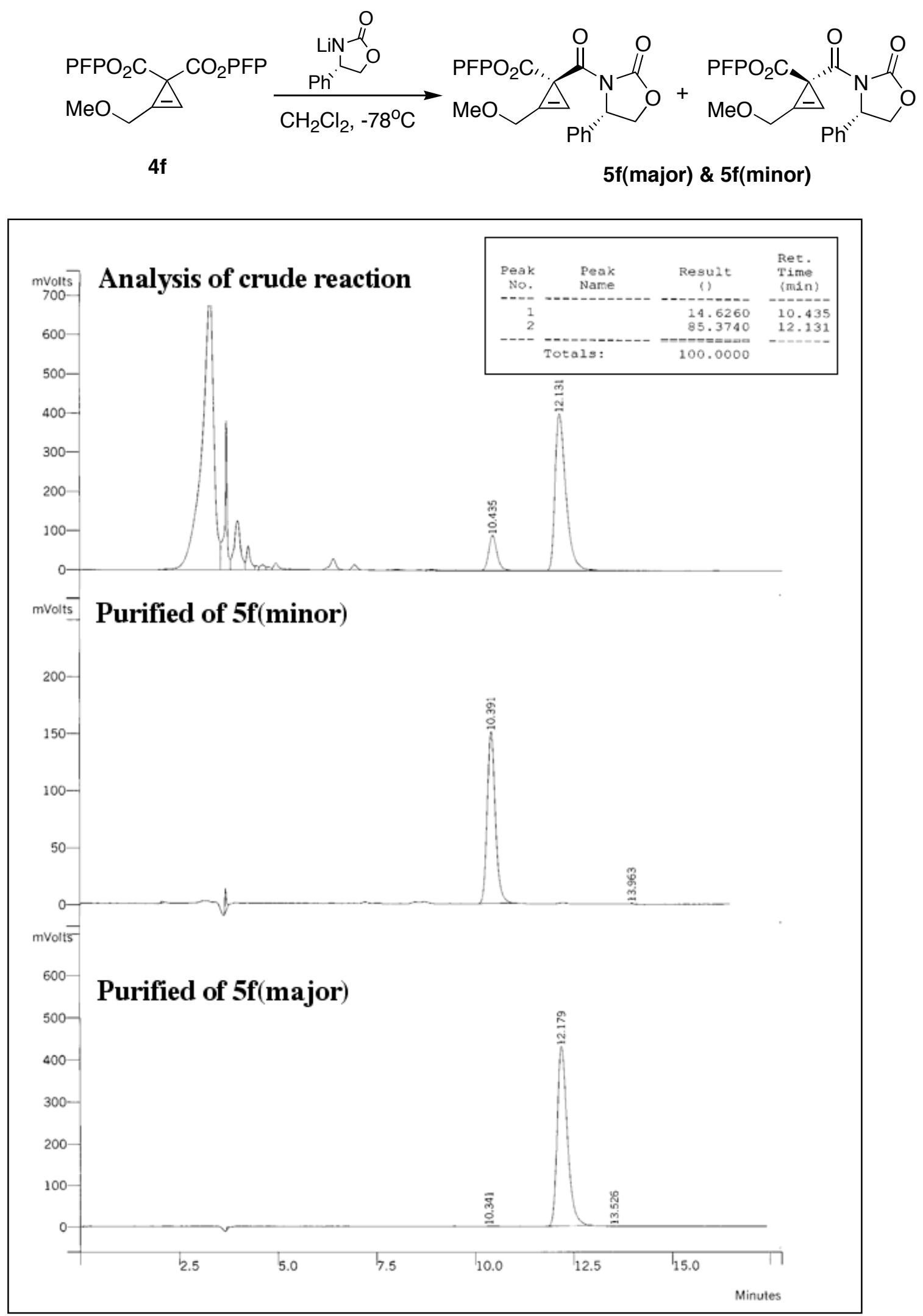
HPLC Data for 5g:

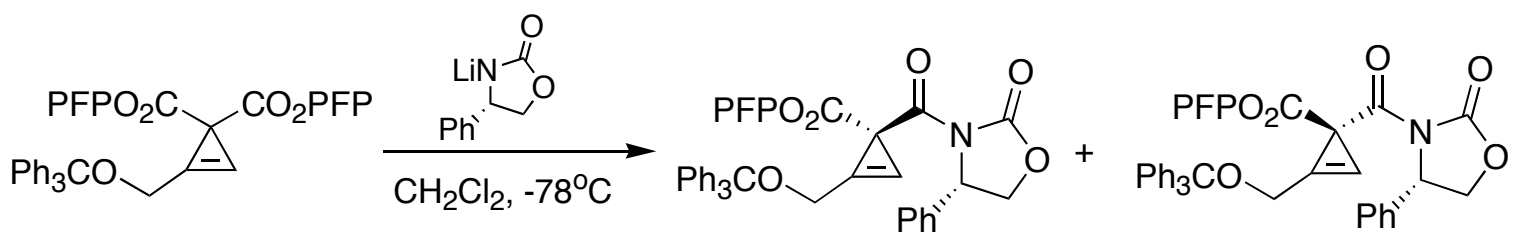

$4 g$

$\mathbf{5 g}$ (major) \& $\mathbf{5 g}$ (minor)

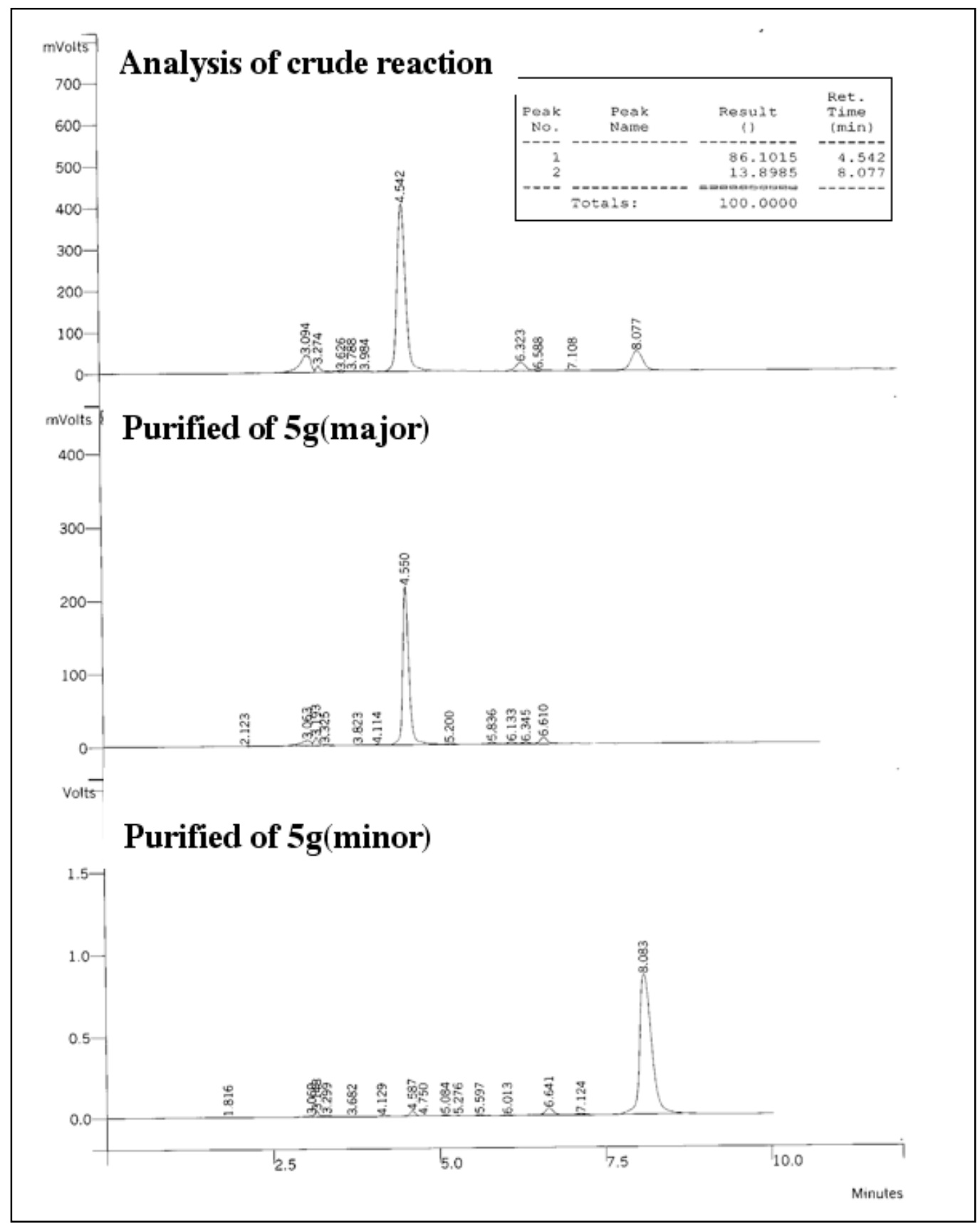


Molecular diagram from x-ray analysis of $\mathbf{5 b}(\boldsymbol{R} \mathbf{c y}, \boldsymbol{S o x})$ depicted with ellipsoids

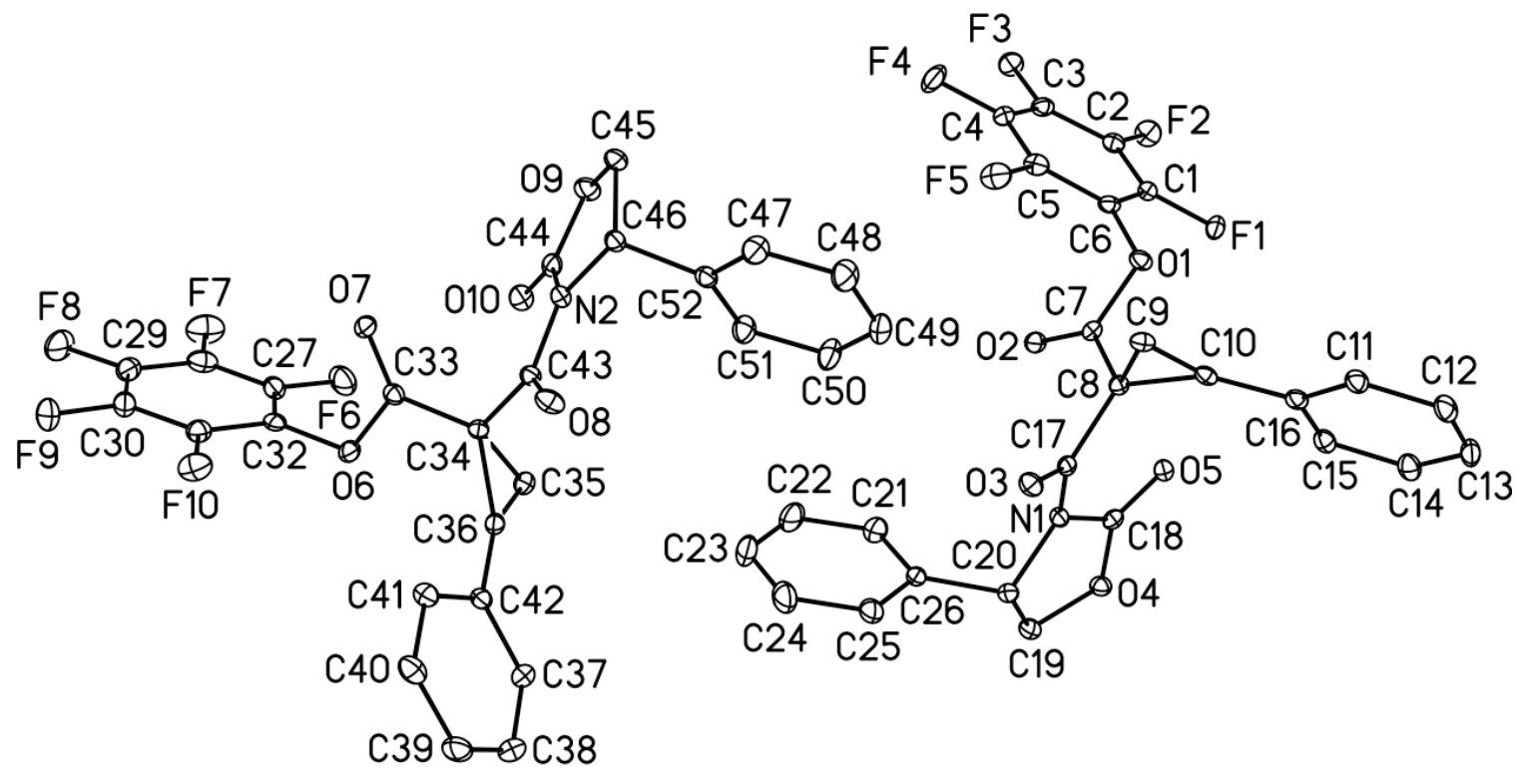

